
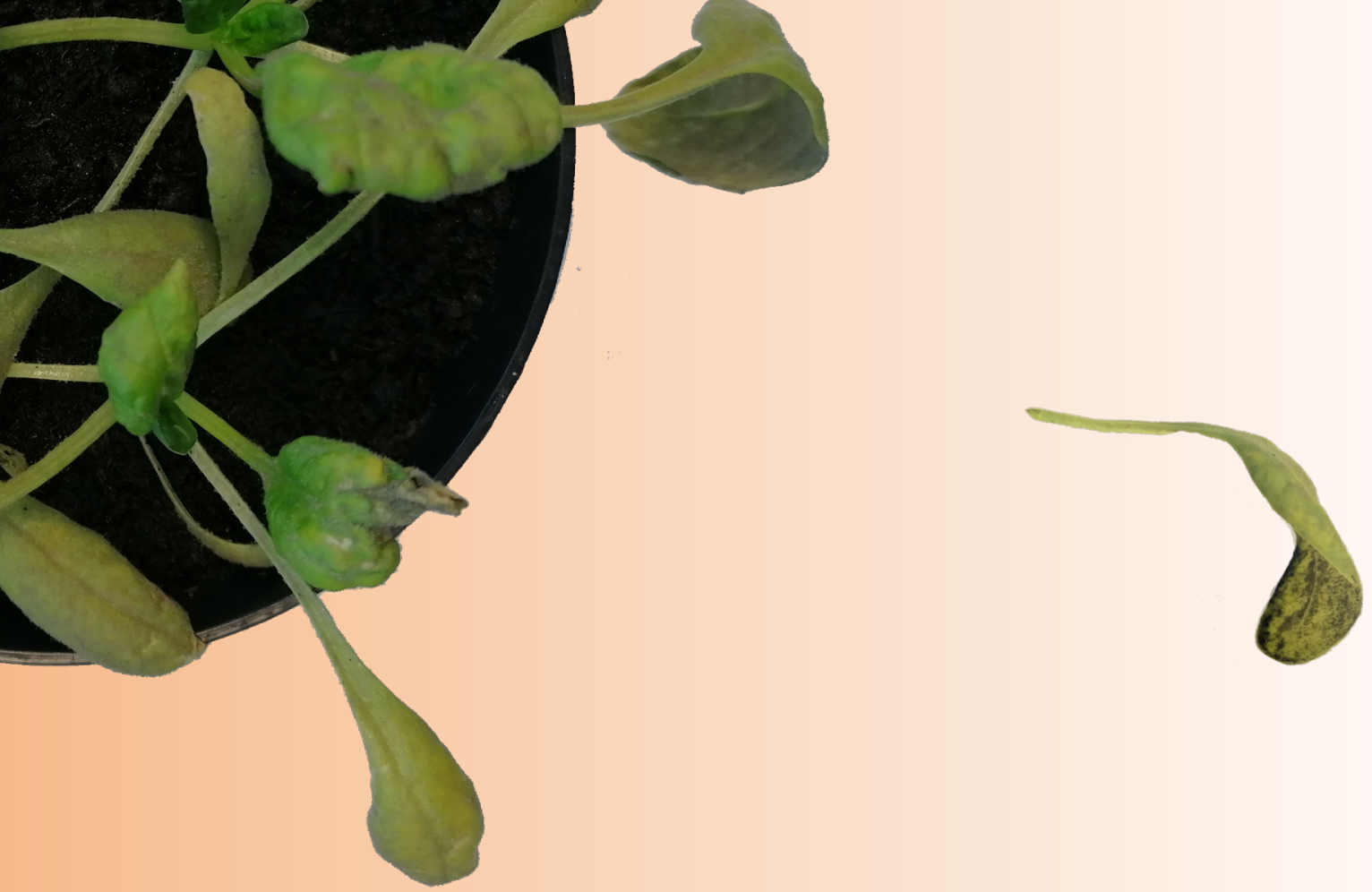

RxLR and CRN effectors of the spinach downy mildew Peronospora effusa and their interacting plant proteins

Manon Neilen 


\section{Reading committee}

Prof. dr. Yuling Bai

Wageningen University, Wageningen, The Netherlands

Dr. Petra M. Bleeker

University of Amsterdam, Amsterdam, The Netherlands

Prof. dr. Ronald Pierik

Utrecht University, Utrecht, The Netherlands

Prof. dr. Martijn Rep

University of Amsterdam, Amsterdam, The Netherlands

Prof. dr. Marco Thines

Goethe University, Frankfurt, Germany

(C) 2020 whattheconcept

ISBN: 978-90-393-7314-9

Layout and design: whattheconcept. Lianne van Rosmalen

Printing: Ridderprint 


\section{RxLR and CRN effectors of the spinach downy mildew Peronospora effusa and their interacting plant proteins}

\section{RxLR en CRN effectoren van de valse meeldauw van spinazie (Peronospora effusa) en de planteneiwitten waarmee ze interacteren. \\ (met een samenvatting in het Nederlands) \\ Proefschrift}

ter verkrijging van de graad van doctor aan de

Universiteit Utrecht

op gezag van de

rector magnificus, prof.dr. H.R.B.M. Kummeling,

ingevolge het besluit van het college voor promoties

in het openbaar te verdedigen op

woensdag 23 september 2020 des middags te 2.30 uur

door

\section{Manon Neilen}

geboren op 14 maart 1990

te Utrecht 


\section{Promotor:}

Prof. dr. A.F.J.M. van den Ackerveken 




Contents
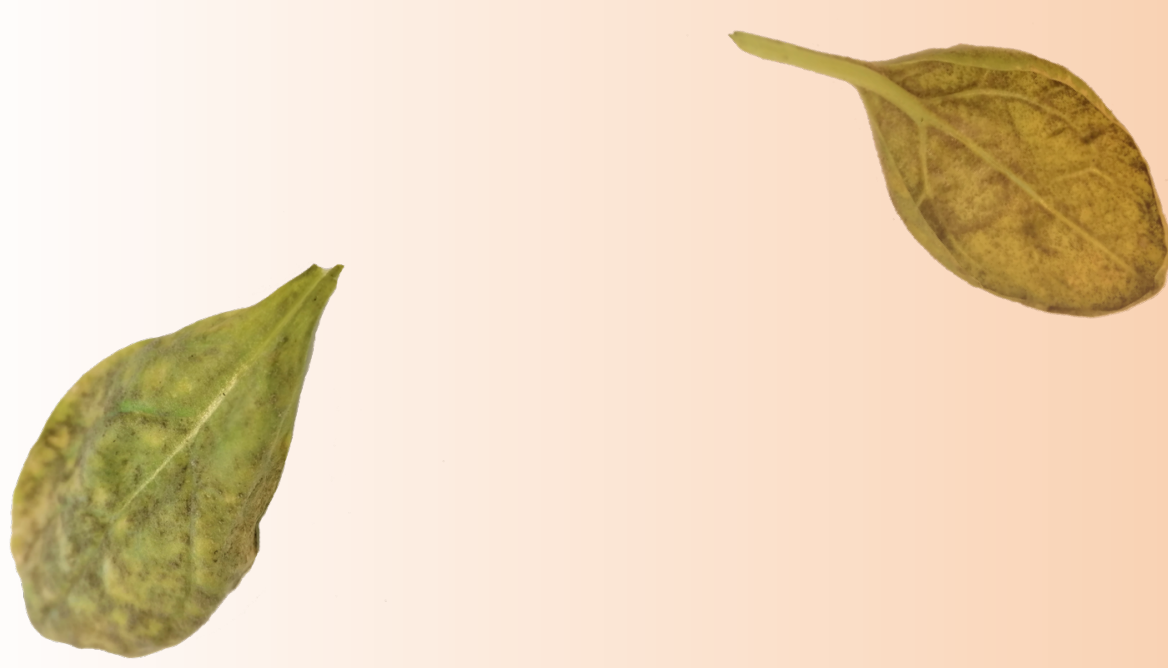
Chapter 1

General introduction

Chapter 2

Genome reconstruction of the

non-culturable spinach downy mildew

Peronospora effusa by metagenome filtering.

Chapter 3

Identification of plant interactors of

host-translocated effectors of Peronospora

effusa.

Chapter 4

Imaging Peronospora effusa effector-host

protein interactions in planta

Chapter 5

General discussion

References

Summary

Samenvatting

Acknowledgement/dankwoord

About the author 244

List of publications 245 


$$
1
$$




\section{1 \\ General}

\section{introduction}

Manon Neilen ${ }^{1}$, Guido Van den Ackerveken ${ }^{1}$

${ }^{1}$ Plant-Microbe Interactions, Department of Biology, Utrecht University, Padualaan 8, $3584 \mathrm{CH}$ Utrecht, the Netherlands 


\section{General introduction}

Plants are confronted with many different microbial pathogens, ranging from viruses to bacteria, fungi and oomycetes. Each in their own way, pathogens interact with the plant to acquire nutrients for growth and reproduction. When a pathogen attack is sensed by a plant it can induce a strong immune response to fight of the pathogen. This ever-changing battleground is a fascinating setting for the study of plant-pathogen interactions, co-evolution and the molecular signaling involved in plant resistance and susceptibility.

The focus in this thesis is on the molecular interaction between the obligate biotrophic oomycete Peronospora effusa (previously known as $P$. farinosa forma specialis spinaciae, and here referred to as Pfs) and its spinach host [1]. Here, I provide a background into the biology and taxonomy of phytopathogenic oomycetes, the lifecycle of Pfs and an overview of the molecular interactions that occur between oomycetes and plants.

\section{Biology and taxonomy of phytopathogenic oomycetes}

Oomycetes are eukaryotic microorganisms that have a filamentous appearance, which resembles the mycelial growth of true fungi. This resemblance is the result of convergent evolution rather than evolutionary kinship because oomycetes and fungi species are very distantly related within the eukaryotes [2, 3]. As opposed to fungi, oomycetes have aseptate hyphae, cell walls composed mainly of cellulose rather than chitin and typically motile biflagellate zoospores (that have been lost in many species) [4]. Oomycetes belong to the Straminipila phylum that includes diatoms and brown algae and hosts species that occupy diverse habitats and have an extraordinary array of lifestyles [5]. In fact, even within the oomycete group, lifestyles and habitats range from freeliving/saprotrophic marine and freshwater species, to human pathogens like Pythium insidiosum and a large number plant pathogenic species (phytopathogens) [6]. 
The ability to be pathogenic on plants evolved multiple times in the evolutionary history of oomycetes. Phytopathogenic species can be found in five different taxa; the Aphanomyces genus, the white blister rusts (Albugo), Pythium, Phytophthora and downy mildew species [7]. The species in each clade have a different lifestyle and infection strategy. Lifestyles vary from necrotrophic (killing cells and feeding on the contents) in Aphanomyces and Pythium species to obligate biotrophic in downy mildew and Albugo species that strictly require living host tissue to grow and fulfill their life cycle. Phytophthora species are hemi-biotrophs that transition from an initial biotrophic to a necrotrophic phase when the infection progresses [8].

Phytopathogenic species of the early diverging Aphanomyces genus are necrotrophic pathogens of seedlings and plant roots [9] that spread by producing mobile zoospores in the soil. They have a sexual and asexual reproduction cycle and are found on a wide range of plants including crop and ornamental species like Iris hollandica, Spinacia oleracea and legume species, e.g. on Medicago [10]. White blister rusts in the Albuginales order are a group of obligate biotrophic species that infect angiosperms. They are phylogenetically distant from the Peronosporales species and likely evolved obligate biotrophy independently of those species [7]. They primarily reproduce asexually via motile flagellated zoospores that are released from zoosporangia. The zoospores settle in stomata and form a germ tube and subsequently hyphae that grow intercellularly and penetrate plant cells [11]. To fulfill their life cycle they produce zoosporangia that rupture the plant epidermis resulting in characteristic white blisters [12]. Sexual reproduction, that enables recombination providing increased genetic diversity, results in the formation of oospores that can survive harsh conditions [13]. Albugo species differ in host range; some species like Albugo candida occur on a wide range of Brassicaceae species while others like Albugo laibachii are found on Arabidopsis thaliana only [14]. Albugo species were shown to exhibit remarkable defense suppression capacity [15]. Plants infected with Albugo are more susceptible to co-infection and to infection with 
incompatible pathogens, including avirulent strains of the downy mildew Hyaloperonospora arabidopsidis. The molecular mechanisms behind 1 Albugo mediated suppression of non-host resistance against Phytophthora infestans may involve manipulation of tryptophan metabolism resulting in an decrease of tryptophan-derived microbial compounds [16]. The other groups of phytopathogenic oomycetes all occur within the large and diverse order of Peronosporales and include saprotrophic, necrotrophic, hemi-biotrophic and obligate biotrophic genera [17].

Within the Peronosporales order the Pythiaceae form the basal lineage to the Peronosporaceae that encompass the Phytophthora and downy mildew species [18]. One has to note that other authors classify the Pythiaceae in a separate order, Pythiales, rather than as a family within the Peronosporales order [19]. Species in the genus Pythium have a diversity of host-ranges, varying from pathogens of marine red algae to pathogens of animals. Phytopathogenic Pythium species are generalist facultative necrotrophic phytopathogens that affect all parts of the plant, including seeds and sprouts [18, 20]. Phytopathogenic Pythium species grow filamentously and do not develop haustoria, feeding structures that are found in Phytophthora and downy mildew species. Like Aphanomyces, Pythium primarily spreads through zoospores in the soil but unlike Aphanomyces also in water [8]. No differential responses of host cultivars to Pythium infection have been observed, suggesting that gene-for-gene interactions do not take place in contrast to what has been often found for Phytophthora and downy mildew species [18].

By far the highest number of phytopathogenic oomycete species belong to the Peronosporaceae, the other Peronosporales group. This large family includes the remorseless Phytophthora species and several groups of downy mildew species. One of the most infamous members is $P$. infestans, the causal agent of potato late blight that led to the death of around one million people during the Great Irish famine around 1845 [21]. The downy mildew genera also cause diseases that are of great economic importance, like Plasmopara viticola on grapevine and Pfs on spinach $[22,23]$. 
Until recently, Phytophthora and the downy mildew species were considered sister groups based on morphological and biological traits [6, 24]. Biologically, downy mildew species are obligate biotrophs that fully depend on living tissue for their survival and are usually host species or genus specific. Phytophthora species are hemi-biotrophs that after an initial biotrophic phase kill the host and are able to grow off death tissue. Most Phytophthora species have a broader host range than downy mildews. Morphologically, hyphae of downy mildew species are terminated when forming the spore bearing sporangiophores whereas Phytophthora hyphae continue to grow [25]. However, with the growing number of species studied, it became clear that these characteristics were not so strictly separated between the two phyla as species with intermediate characteristics were identified [25, 26].

These initial observations of morphological traits that are not strictly separated between Phytophthora and downy mildew species were later supported by genetic evidence. Studies using multiple genes or full genome comparisons suggested that the downy mildews not only reside within, but also have multiple independent origins in the larger Phytophthora clade [19, 22, 27]. This means that the obligate biotrophic lifestyle emerged at least two times independently within the Peronosporales order. One downy mildew clade encompasses the majority of downy mildew species. It includes graminicolous grassinfecting downy mildews, the downy mildews with colored conidia and the brassicolous downy mildews [27]. The downy mildews with colored conidia encompass the largest downy mildew genus Peronospora and the genus Pseudoperonospora that both have purple to black sporangia [17]. The second clade contains downy mildew species with pyriform haustoria that has recently been shown to be more closely related to Phytophthora clade 1 than to the other downy mildew species [22]. Downy mildews with pyriform haustoria includes eight genera, which are almost exclusively infecting Asteraceae, including crops like lettuce (Bremia lactucae) [17, 28]. The exception is the genus Plasmopara that infects other species like grapevine ( $P$. viticola) and sunflower ( $P$. halstedii) $[22,29]$. 
Pfs, the causal agent of downy mildew on spinach, belongs to the Peronospora family. Downy mildew is a major disease in commercial 1 spinach fields worldwide and restrains both crop quality and quantity [30]. Pfs was first reported in 1824 [31] and, following the introduction of resistant spinach cultivars, new isolates evolved rapidly over the last two decades [23, 32].

\section{Life cycle of Peronospora effusa, the downy mildew of spinach}

Pfs has a sexual as well as an asexual life cycle. The asexual sporangiospores germinate when they have landed on plant tissue. The emerging germ tubes develop to penetrate into plant tissue and initiate the infection [30]. Hyphae continue to grow in the tissue between the plant cells. Specialized hyphae form haustoria that are thought to be the site of interaction between oomycete and the plant cell (discussed in the following section). The hyphae form terminal sporangiophores, branched spore-bearing structures that grow out of the stomata and contain the sporangia that are visible on infected leaves as a grey furry layer [25] (Fig. 1). The sporangia travel aerially and through rain splash and facilitate rapid spread of $P f s$ [30].

During sexual reproduction in gametangia thick-walled oospores are produced that genetically represent a combination of both parental genomes. Pfs is heterothallic, i.e. parental lines (P1 and P2) have to be of a different mating type for sexual reproduction to occur [33, 34]. Sexual recombination can generate extensive genetic variation in a pathogen population. As opposed to sporangia, the microscopic oospores do not occur on the leaf surface but inside plant tissue, below the epidermal layer [30]. Oospores have also been observed in roots and seeds and it was recently shown that Pfs can be transmitted through seeds [35]. Further research on survival and germination of oospores in seed lots is required to understand its contribution to spread of $P f s[30]$. 


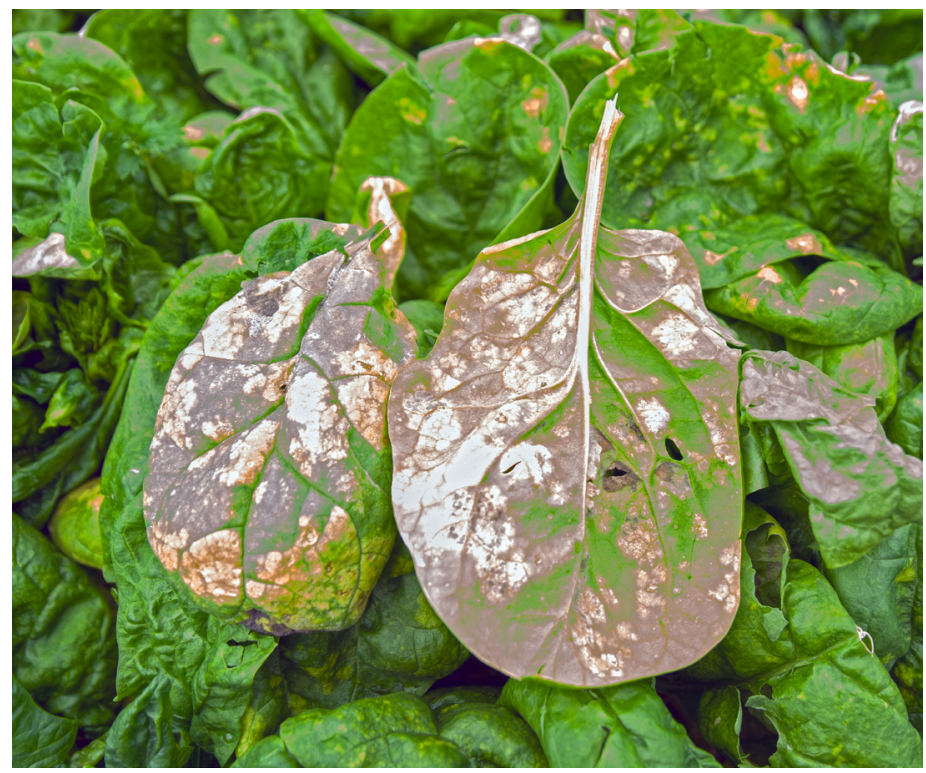

Figure 1. Spinach leaves infected with Peronospora effusa.

The visible symptoms include irregular chlorotic spots and gray to brown sporangial masses, primarily on the underside of the leaf. The symptoms develop between seven to ten days post infection [30]. Picture taken by Pim Goossens.

\section{Molecular interaction with the plant}

Peronosporales species interact with plant cells at specialized feeding structures called haustoria. 'Haustorium' literally means 'drawer of water' which is illustrative of the presumed function of the structure in acquisition of water and nutrients from the plant [36]. To establish an haustorium, the cell wall of the plant cell is locally degraded so that the plasma membrane can be invaginated by the haustorium [37]. The oomycete remains separated from the host cell by the extrahaustorial matrix (EHMx) and the extrahaustorial membrane that is continuous with the plasma membrane. The extrahaustorial matrix contains material from both plant and pathogen origin [38].

The oomycete derived materials in the EHMx are both actively secreted and derivatives of the oomycete cell wall and cell membrane [39]. Many pathogenesis-related proteins of the plant host are hydrolytic enzymes (e.g. glucanases and proteases) that break down cell walls and membrane components that then release into the EHMx [40]. These 
components can serve as pathogen-associated molecular patterns (PAMPs); potent triggers of basal plant immune responses. PAMPs are recognized by membrane-bound pattern recognition receptors (PRRs) that then activate a series of anti-microbial defenses like callosedeposition, production of reactive oxygen species (ROS), release of antimicrobial compounds, alteration of hormone signaling and changes in gene expression. These collectively contribute to pattern-triggered immunity (PTI) [41, 42, 43].

Cell wall derived $\beta$-glucan oligomers are an example of PAMPs in the recognition of both fungal and oomycete pathogens in many plant species, although the mechanisms are not fully understood [44, 45]. $\beta$-glucan oligomers are insoluble polysaccharides that have a structural role in cell walls [46]. They are the most abundant cell wall components in oomycetes [45]. Unlike the elicitor response triggered by chitin, $\beta$-glucan elicitation of defense is not conserved between plants and different species respond differently. This observation is possibly explained by the large diversity of $\beta$-glucan structure between species of oomycetes and fungi [47]. Other examples of oomycete cell wall and membrane derived PAMPs are transglutaminases and fatty acids [39].

\section{Apoplastic effectors}

Oomycetes secrete proteins into the EMHx during infection [44]. Pathogen-secreted proteins that help to establish and maintain infection are called effectors. An important function of effector proteins is to avoid or suppress pathogen recognition and subsequent immune responses of the plant. Effector proteins can either function in the apoplast or inside host cells. Apoplastic effectors include protease- and glucanaseinhibitors, elicitins and necrosis and ethylene-inducing peptide 1 like proteins (NLPs), among others. Protease and glucanase inhibitors function to protect the oomycete against damage induced by hydrolytic enzymes of the plant, thereby also reducing the release of PAMPs [40]. An example of apoplastic effectors with protease inhibitor function are the EpiC effectors of $P$. infestans that inhibit plant papain-like cysteine proteases [48]. 
Elicitins are small secreted proteins that induce necrosis and defense hormone signaling in plant species from a wide range of plant families $[49,50]$. They are possibly involved in acquiring plant sterols, compounds that Phytophthora and downy mildew species are unable to synthesize themselves [49].

NLPs are present in the secretomes of plant-associated bacteria, fungi and oomycetes [51]. Plant-infecting oomycetes have large expansions of NLP genes, suggesting they play an important role in the phytopathogenic lifestyle $[52,53]$. Their name is derived from their ability to induce necrosis in eudicots as a result of their cytotoxicity. However, in many species also non-cytotoxic NLPs have been identified. The numbers of cytotoxic NLPs are highly expanded within Phytophthora species but not in the obligate biotrophic $H$.arabidopsidis. In fact, the genome of $H$. arabidopsidis encodes 10 NLPs that are all non-cytotoxic [52]. As opposed to cytotoxic NLPs, the non-cytotoxic ones are expressed during the early stages of infection, suggesting a role in penetration or early colonization of the host $[39,52,54]$.

\section{Host-translocated effectors}

In addition to effectors that function in the apoplast, oomycetes produce proteins that are known to be translocated across the plant cell membrane into plant cells. Two types of oomycete host-translocated effectors are currently known, the RxLR and CRN effectors [55, 56]. RxLR and CRN effectors manipulate plant processes, like immune signaling, and protein transport, to the benefit of the oomycete [57]. CRN effectors are thought the be ancient effectors because they are found in all phytopathogenic oomycete species, including the basal root pathogen $A$. euteiches, of which protein AeCRN5 was shown to possess a domain that is able to facilitate host translocation [56]RxLR effector proteins were originally identified in $\mathrm{H}$. arabidopsidis and Phytophthora genomes [55]. Especially Phytophthora species have largely expanded sets, adding up to hundreds of secreted RxLR effectors per species [58]. Large repertoires of 100 to 200 RxLRs were also identified in other downy mildew species [29, 59, 
$60,61]$. One notable exception is the downy mildew of basil (Peronospora belbahrii) that only has $22 \mathrm{RxLR}$ like proteins [62]. Smaller numbers of

1 RxLR effector proteins have been reported from Pythium and Albugo but not from other oomycete species. The contribution of RxLR effectors to virulence in Albugo and Pythium is not clear although some preliminary hints for a role of Pythium and Albugo RxLR-like effectors in virulence have been found. For example, it was demonstrated that $P$. syringae $p v$. tomato DC3000 harboring Albugo laibachii RxLR effector 1 and 2 were more virulent on plants compared to wildtype Pst DC3000 and RxLR effectors of Pythium species can induce cell death when expressed in $N$. benthamiana, pointing at recognition of the effectors by the plant immune system [63, 64].

RxLR and CRN effectors are modular proteins that are characterized by the presence of a signal peptide at the N-terminus, a presumed hosttranslocation domain and a variable $\mathrm{C}$-terminal part, called the effector domain, which executes the function of the effector in the plant cell. The RxLR translocation domain is characterized by a RxLR-EER (arginine, any amino acid, leucine arginine) domain that is thought to be required for translocation of RxLR effectors [65, 66, 67]. Mutation of the RxLR-EER motif of P. infestans effector Avr3a was shown to prevent translocation of the protein into the host cell [65]. The means by which the RxLR effectors are translocated are not yet fully understood. Unlike bacterial phytopathogens, oomycetes (and fungi) have no known translocation system like the type III secretion system that injects bacterial proteins into the host cell. Recently, it was demonstrated that delivery of RxLR effector Pi04314 was independent of the conventional ER-to Golgi secretion system. Infiltration with brefeldin A, a fungal compound that interferes with Golgi-vesicle formation, did not inhibit translocation of Pi4314 into N. benthamiana cells [68, 69]. Golgi-independent translocation was also observed for effectors of the fungal pathogen Magnaporthe oryzae [70]. The unconventionally secreted signal peptidecontaining proteins are thought to be transported from the ER directly to the plasma membrane, without passing through the Golgi [69, 71]. 
Ultrastructural studies revealed the presence of exosomes/vesicles in the EHMx and multivesicular bodies within fungal and plant cells [72, 73]. Potentially, unconventional secretion of oomycete and fungal effectors may be mediated by extracellular vesicles that are released upon fusion of multivesicular bodies with the oomycete plasma membrane [74]. The extracellular vesicles may then deliver their cargo into plant cells by fusing to the host cell-or extrahaustorial membrane [71].

In CRN effectors, the signal peptide is followed by a LxFLAK motif, DWL domain and HVLVXXP motif $[58,75]$. Protein fusions between $\mathrm{N}$-termini of CRN2, CRN16, CRN8 and the effector domain of $P$. infestans RxLR effector Avr3a, transgenically expressed in $P$. capsici were shown to translocate into $N$. benthamiana cells, triggering a defense response. This suggests that the $\mathrm{N}$-termini, including the LFLAK domain are important for translocation [56]. The means by which the CRN effectors are translocated are not yet understood.

The C-terminal effector domains are responsible for the virulence function of the host-translocated effectors and usually lack known functional domains and show little sequence conservation between species. However, about $44 \%$ of the Phytophthora RxLR effectors do share a conserved tertiary structure, an a-helical fold, termed the WYfold (Trp (W) and Tyr (Y)). WY-folds were found to occur as individual units, duplications, dimers and tandem repeats in different RxLR proteins [76]. Due to the a-helical orientation functionally important and polymorphic residues are presented at the surface of the proteins [77]. It is hypothesized that the WY-folds provide molecular stability to the proteins while maintaining functional variability of the effector domain. The structure may llow the evolution of effectors to maintain their function while evading recognition by the plant immune system [77]. Effectors with WY-folds were thus far implicated in various processes such as RNA silencing suppression (Phytophthora suppressor of RNA silencing 2 (PSR2, P. sojae) and manipulation of vesicular trafficking (PexRD54, P. infestans) [78, 79]. 
Plants have evolved intracellular receptors that recognize effectors or their activity. Recognition is mediated by nucleotide-binding domain containing leucine-rich repeat receptors (NB-LRR) that directly or indirectly interact with the effector proteins and trigger an immune response termed effector-triggered immunity [80]. As opposed to PAMPs, effectors are variable and dispensable resulting in co-evolution and large diversification of both effectors and intracellular receptors. The cellular responses triggered by ETI are similar to those triggered by $\mathrm{PTI}$, but stronger and faster and are often associated with localized cell death (the hypersensitive response (HR)) [80].

\section{Plant processes manipulated by oomycete effectors}

Host-translocated RxLR and CRN effectors are potent manipulators of a plethora of plant cell functions to enable infection of the host. Although the molecular function of the majority of effectors remains unknown, efforts in recent years have led to the identification of the molecular function of a growing number of oomycete effectors (Table 1). The host-translocated effectors manipulate processes as broad as cellular programming (through immune-and hormone signaling and direct manipulation of transcription/ translation), PAMP-induced production of ROS, and vesicular trafficking (Fig. 2). In addition, effectors can suppress ETI, e.g. by the P. sojae CRN effector PsCRN70 that suppresses PsAvh421, PsCRN63 and Avr3a-induced cell death. However, the molecular mechanism is not yet understood [81]. Examples of manipulation of each of the above mentioned processes by an oomycete effector are detailed in the following paragraphs. 







\begin{tabular}{|c|c|c|c|c|c|c|c|c|c|c|c|}
\hline 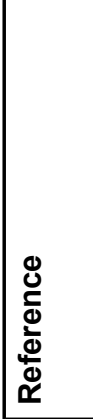 & 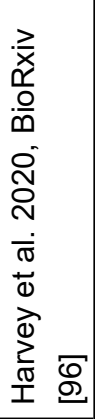 & 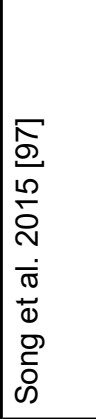 & 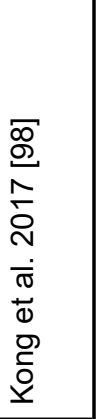 & 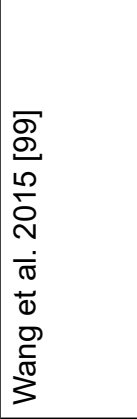 & 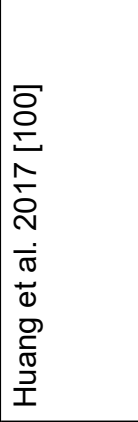 &  & 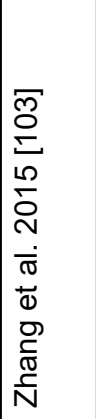 & 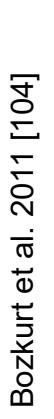 & 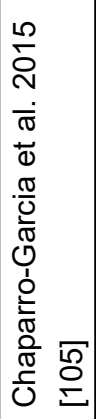 & 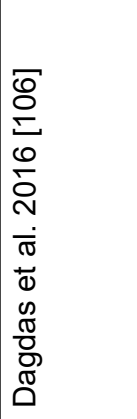 &  \\
\hline 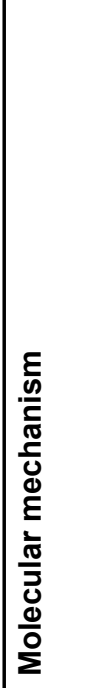 & 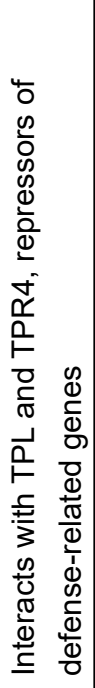 & 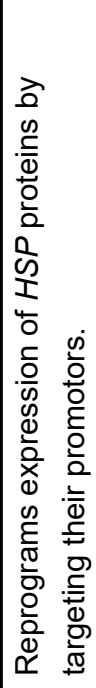 & 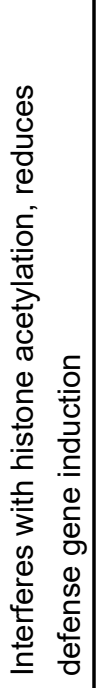 & 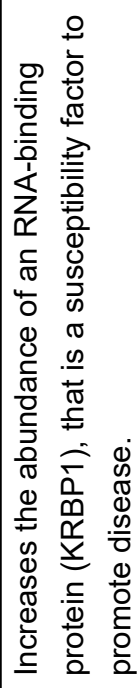 & 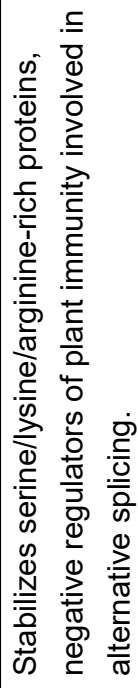 & 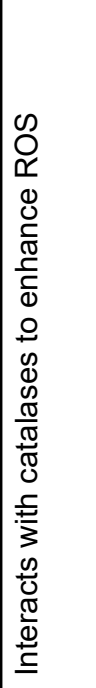 & 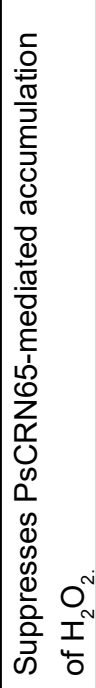 & 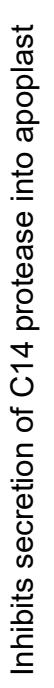 & 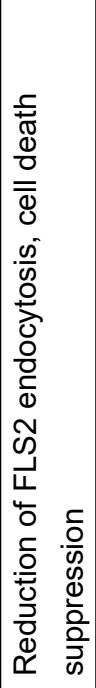 & 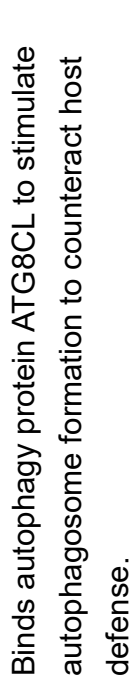 & 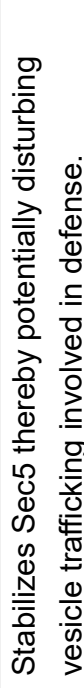 \\
\hline 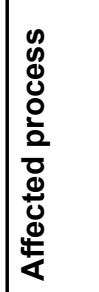 & 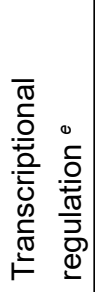 & 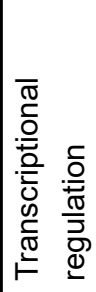 & 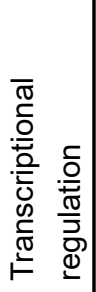 & 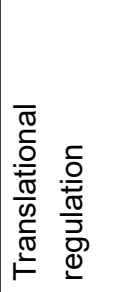 & 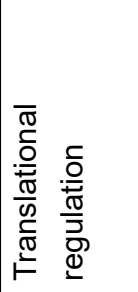 & 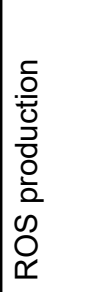 & 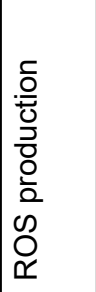 & 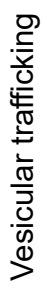 & 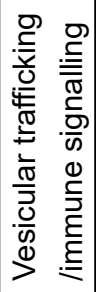 &  & 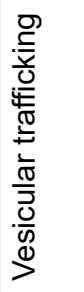 \\
\hline 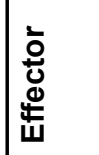 & $\begin{array}{l}\bar{N} \\
\frac{x}{x} \\
\frac{\widetilde{N}}{I}\end{array}$ &  & 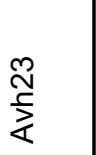 & $\begin{array}{l}\text { D } \\
\text { o } \\
\text { ᄋ }\end{array}$ & ల్ల & 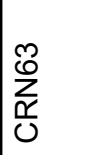 & $\begin{array}{l}\frac{10}{\Sigma} \\
\underset{\sim}{Z} \\
\frac{\alpha}{U}\end{array}$ & $\frac{\rho}{c}$ & 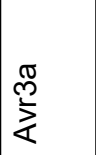 & $\begin{array}{l} \pm \\
0 \\
0 \\
\frac{1}{x} \\
0 \\
0\end{array}$ & 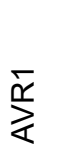 \\
\hline 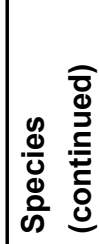 & 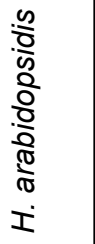 & $\begin{array}{l}\frac{\pi}{2} \\
\frac{\pi}{2} \\
0\end{array}$ & $Q$ & $\begin{array}{l}\mathbb{\Phi} \\
\mathbb{D} \\
\mathbb{Q}\end{array}$ & $\begin{array}{l}0 \\
0 \\
\infty \\
0 \\
0\end{array}$ & $\begin{array}{l}-\frac{\pi}{2} \\
-8 \\
0 \\
0\end{array}$ & $\begin{array}{l}\frac{\pi}{0} \\
\frac{\pi}{2} \\
0 \\
0 .\end{array}$ & & 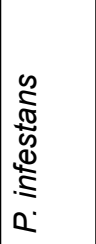 & 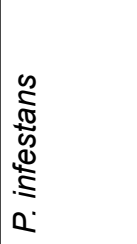 & 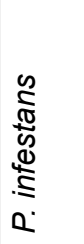 \\
\hline
\end{tabular}


a Alternative names: Pi06087, PexRD16

${ }^{b}$ ER-luminal binding immunoglobulin proteins (BiPs)

c1-aminocyclopropane-1-carboxylate synthase (ACS)

${ }^{d}$ Orthologs of PSR1 from P. parasitica (PpPSR1L) and P. capsici (PcPSR1L) possess the same function

${ }^{e}$ Hypothesized function, further research is needed. TPL $=$ Topless, TPR4

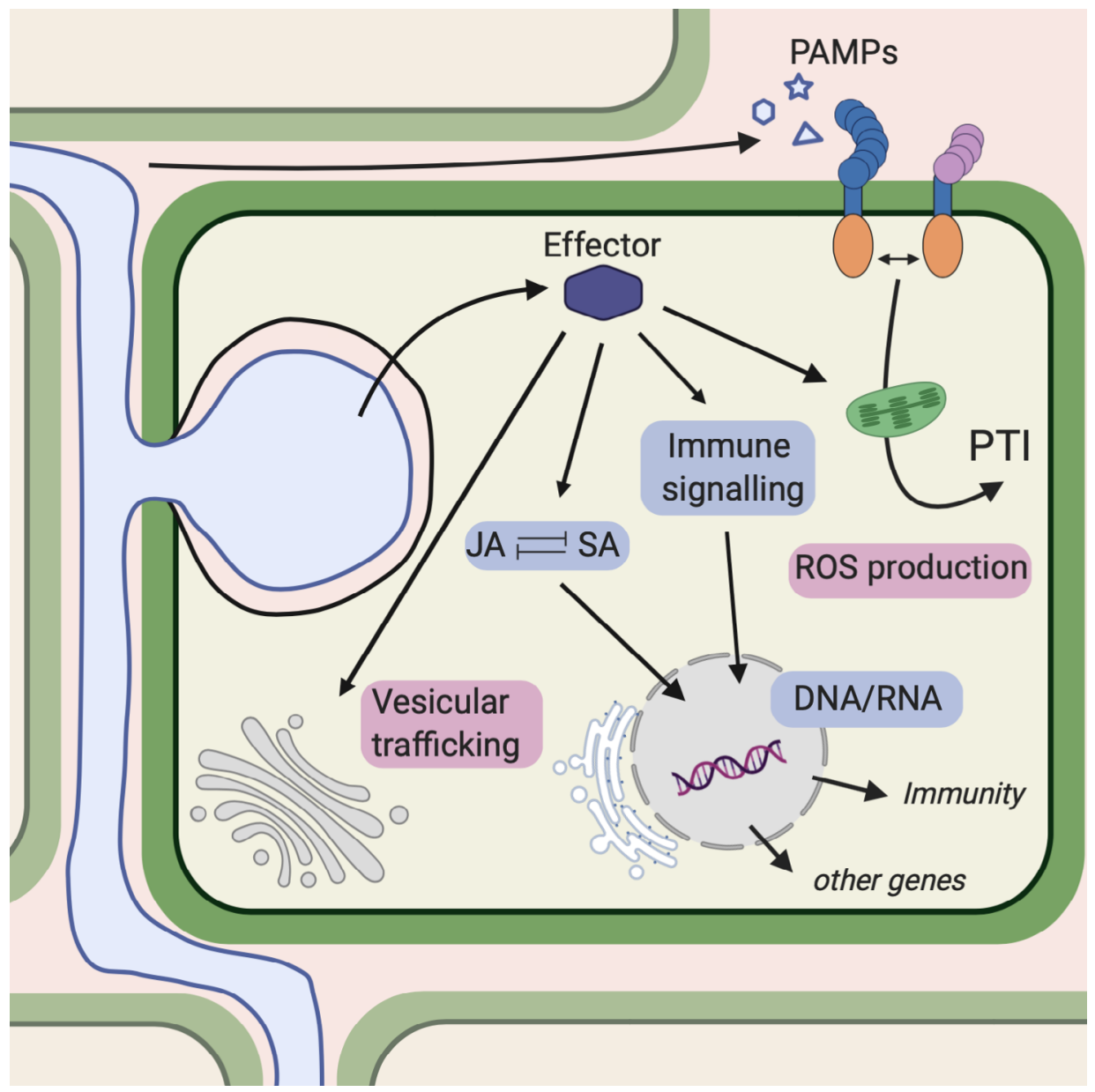

Figure 2. Plant processes manipulated by oomycete effectors

Phytophthora and downy mildew pathogens interact with plant cells at specialized feeding structures called haustoria. Two types of effectors, RxLR and CRN effectors, are translocated into plant cells. Oomycete effectors have been shown to manipulate vesicular trafficking, ROS production and cellular programming (italics). Manipulation of cellular programming occurs by interfering with immune and defense hormone signaling and transcription and translation. The anticipated effector-mediated suppression of ETI is not indicated because no molecular mechanism of manipulation by an oomycete effector has described to our knowledge. 


\section{Cellular programming: Immune signaling}

$P$. infestans effector Pi02860 was found to repress immune signaling 1 through indirect manipulation of SWAP70, a positive regulator of infestin 1 (INF1)-triggered cell death [84, 108]. The effector was found to interact with StNRL1 (NPH3/RPT2-like), a predicted substrate adaptor of a CULLIN3-associated ubiquitin E3 ligase [109]. Silencing of the $N$. benthamiana ortholog of NLR1 abolished the ability of Pi02860 to suppress cell death and led to reduced plant colonization by $P$. infestans. These observations suggest that NRL1 is a susceptibility factor that negatively regulates immunity. NLR1 homodimers were found to bind SWAP70, a guanine exchange factor that positively regulates cell death, and target it for proteasome-mediated degradation. By interacting with NRL1 P. infestans effector Pi02860 enhances the turnover of SWAP70, thereby suppressing the cell death response [84].

\section{Cellular programming: Hormone signaling}

The tight and accurate regulation of plant immune responses is regulated by defense hormones. Generally, salicylic acid is induced upon infection with biotrophic pathogens while jasmonic acid and ethylene are upregulated when a plant is attacked by a necrotrophic pathogen or herbivore. These opposite hormonal signaling networks are known to antagonize each other to optimally arm the plant to fight off the current attacker. The crosstalk between the hormone pathways is exploited by a RxLR effector of $H$. arabidopsidis. Effector HaRxL10 was shown to interact with JAZ3, a transcriptional repressor involved in jasmonic acid signaling, and to target it for proteasome-mediated degradation. Degradation of JAZ3 led to enhanced jasmonic acid signaling and eventually reduced SA-signaling mediated by the release of the JAZ3-guarded MYC2 transcription factor. Arabidopsis plants expressing HaRxL10 also showed enhanced susceptibility to the biotrophic bacterium Pseudomonas syringae pv. DC3000 compared to Col-0 [93]. 


\section{Cellular programming: transcriptional and posttranscriptional manipulation}

Cellular reprogramming by oomycete effectors can occurs through gene regulation [86] and post transcriptionally through alternative splicing [100] and interference with RNA silencing [110]. P. sojae RxLR effector PsAvh23 was found to reduce histone acetylation levels thereby suppressing the activation of defense genes [98]. DNA in cells is organized around nucleosomes, that consist of an octamer of basic proteins called histones. Acetylation of the tails of these proteins by histone acetyltransferases (HATs) is associated with enhanced gene transcription whereas histone deacetylases (HDACs) reverse the modification and repress gene transcription [111] In plants, the HAT General Control Non-depressive 5 (GCN5) is part of the prototypical nucleosome-acetylating modification complex. GCN5 is modulated by Alteration/Deficiency IN Activation 2 (ADA2) that directs the complex to the histone H3-tail. PsAvh23 competitively binds to ADA2 leading to reduced HAT activity of GCN5 hence impairing the activation of defense-related genes. Possibly, the known ability to suppress cell death is a result of this manipulation [98].

\section{ROS production}

Two highly similar $P$. sojae CRN effectors, PsCRN65 and PsCRN115 were, surprisingly, found to induce opposite responses in N. benthamiana when expressed in planta. Both effectors interact with catalases, proteins that degrade $\mathrm{H}_{2} \mathrm{O}_{2}$ which is important for activation of cell death [112]. PsCRN65 promoted $\mathrm{H}_{2} \mathrm{O}_{2}$ accumulation and hence cell death whereas PsCRN15 suppresses the PsCRN65-mediated accumulation and cell death [103]. Expression of the individual CRN effectors with NbCAT1 induced subcellular relocalization of the catalase to the nucleus. Both CRN proteins were found to be required for full virulence [101]. 


\section{Vesicle trafficking}

Vesicle trafficking is an important process in the plant immune response

1 [113]. The plant secretory system (exocytic) pathway is required for delivery of antimicrobial proteins and compounds involved in the first layer of defense. It is not surprising that this important defense process is targeted by oomycete effectors. $P$. infestans effector AVR1 binds to and stabilizes Sec5, a subunit of the plant exocyst complex. Expression of AVR1 in $N$. benthamiana reduced callose deposition and enhanced susceptibility to $P$. infestans, a phenotype that can be mimicked by the silencing of Sec5 [107]. In addition, endocytosis is implicated in the regulation of PAMPtriggered and effector-triggered immunity. The Arabidopsis receptorlike kinase FLAGELLIN SENSITIVE 2 (FLS2) recognizes and binds the flagellin-derived peptide flg22 [114]. Upon binding a MAP kinase cascade is activated that induces defense gene transcription. At the same time the flg22-bound (activated) FLS2 receptors are internalized in endosomal compartments and likely targeted for lysosomal degradation [115]. $P$. infestans effector Avr3a was found reduce internalization of activated FLS2 and suppress flg22-triggered immunity. AVR3a associates with a plant GTPase (Dynamin-Related protein 2 (DRP2) that is required for ligand-induced FLS2 internalization [105].

Over the past ten years many oomycete genomes have been sequenced and assembled. These genomes are a valuable source of information that can be used in understanding the biology of plantpathogen interactions. The molecular mechanisms by which effectors manipulate host plant processes are now beginning to be revealed. The positioning of host-translocated effector proteins at the interface between pathogens and plants provides a unique opportunity to study both pathogen infection strategies and the plant immune system. 


\section{Thesis Outline}

The aim of my research described in this thesis was to identify and characterize host-translocated RxLR and CRN effector proteins of $P$. effusa (Pfs), the downy mildew that infects spinach plants. RxLR and CRN effectors are translocated into plant cells, where they manipulate plant processes to establish and maintain a successful infection. Manipulation occurs by binding of effectors to plant proteins, thereby altering their function or affecting their stability. In the introductory chapter above I discussed the biology of oomycete plant pathogens and their molecular interaction with host plants (Chapter 1 ).

Identification the effector repertoire of Pfs starts with the sequencing and assembly of its genome. Due to the obligate biotrophic nature of this pathogen, DNA can only be isolated from infected spinach leaves. DNA from bacteria and fungi that live on those leaves ends up in the isolated DNA, complicating the assembly of the genome. I describe the usage of the CAT tool, originally developed for filtering bacterial genomes, to assign taxonomy to each open reading frame on an assembled contig, aiding the selection of $P f s$-derived contigs. The assembled genome was used to predict the subset of genes that encode secreted proteins that are in direct contact with the plant cell during infection. The secretome of Pfs was compared to that of other plant pathogenic oomycete species to study differences and similarities in functionality of secretomes of species that have different lifestyles and taxonomy (Chapter 2).

The identified candidate RxLR and CRN effectors were studied in more detail in relation to their occurrence and evolution. RXLR and $\mathrm{CRN}$ effectors have a modular organization, characterized by a relatively conserved $\mathrm{N}$-terminus that has a signal peptide and translocation domain and a diverse $\mathrm{C}$-terminal effector domain. The $\mathrm{C}$-terminal effector domain is responsible for the virulence function and usually lacks known protein domains or considerable conservation with other proteins. The lack of conservation is thought to help avoid recognition of the effectors by the plant immune system. Some examples of evolutionary conserved effectors are known, and it is hypothesized that these conserved effectors 
are especially important for oomycete virulence. Evolutionary conserved effectors from different species have similar functions, probably as a 1 result of interaction with conserved plant proteins. Hence, evolutionary conserved effectors provide an opportunity to study plant processes commonly targeted by effectors. In chapter 3 I characterize the Pfs RxLR and $\mathrm{CRN}$ effector repertoire by assessing their evolutionary conservation in other species. We found that of the 99 candidate RxLR and $14 \mathrm{CRN}$ effectors, 39 candidate effectors are specific to Pfs, 10 are conserved between downy mildew species, 23 are conserved in downy mildew and Phytophthora and 40 are more broadly conserved. Note that these numbers total 112, not 113 candidate effectors because one protein has both a canonical RxLR and LFLAK motif and hence qualifies as RxLR as well as $C R N$ candidate effector. A selection of $P f s$ effectors was used for yeast two-hybrid assays to identify interacting plant proteins. This resulted in the identification of the first 40 potential plant targets of $P f s$ effectors (Chapter 3).

Nine high-confidence effectors and their fourteen plant interactors were subsequently selected for functional characterization in plants. The proteins were fused to fluorescent proteins and expressed in $N$. benthamiana to visualize their subcellular localization. We found that the majority of the plant interactors and corresponding effectors localized to the same subcellular compartment when co-expressed, a prerequisite for interaction. Additionally, the subcellular localization of six plant interactors and two effector proteins was altered upon co-expression of the effector and interactor proteins and seven plant proteins showed a more stable signal upon co-expression with the effector Chapter 4). Relocalization and stabilization may result from the interaction with the effector protein. Finally, the evolutionary genomics of oomycete pathogens, specifically downy mildew species, is discussed with emphasis on the evolution of pathogenicity and genome size. Furthermore, the acquired knowledge on the Pfs RxLR and CRN effectors was assessed and used to provide a future outlook on the study of $P f s$ virulence on spinach (Chapter 5). 


$$
P Q
$$




\section{Genome reconstruction of the} non-culturable spinach downy mildew Peronospora effusa by metagenome filtering

Manon Neilen ${ }^{1 \pi}$, Joël Klein ${ }^{1 \pi}$, Marcel van Verk ${ }^{1,2}$ Bas E. Dutilh ${ }^{3}$, Guido Van den Ackerveken ${ }^{1}$

${ }^{1}$ Plant-Microbe Interactions, Department of Biology, Utrecht University, Utrecht, The Netherlands

${ }^{2}$ Crop Data Science, KeyGene, Wageningen, The Netherlands

${ }^{3}$ Theoretical Biology and Bioinformatics, Department of Biology, Utrecht University, Utrecht, The Netherlands

If these authors contributed equally to the manuscript

Published: Klein, Joël, et al. Genome reconstruction of the non-culturable spinach downy mildew Peronospora effusa by metagenome filtering. Plos ONE 15.5 (2020): e0225808. 


\section{Abstract}

Peronospora effusa (previously known as $P$. farinosa $f$. sp. spinaciae, and here referred to as $P f s$ ) is an obligate biotrophic oomycete that causes downy mildew on spinach (Spinacia oleracea). To combat this destructive many disease resistant cultivars have been bred and used. However, new Pfs races rapidly break the employed resistance genes. To get insight into the gene repertoire of Pfs and identify infection-related genes, the

2 genome of the first reference race, Pfs1, was sequenced, assembled, and annotated. Due to the obligate biotrophic nature of this pathogen, material for DNA isolation can only be collected from infected spinach leaves that, however, also contain many other microorganisms. The obtained sequences can, therefore, be considered a metagenome. To filter and obtain Pfs sequences we utilized the CAT tool to taxonomically annotate ORFs residing on long sequences of a genome pre-assembly. This study is the first to show that CAT filtering performs well on eukaryotic contigs. Based on the taxonomy, determined on multiple ORFs, contaminating long sequences and corresponding reads were removed from the metagenome. Filtered reads were re-assembled to provide a clean and improved Pfs genome sequence of $32.4 \mathrm{Mbp}$ consisting of 8,635 scaffolds. Transcript sequencing of a range of infection time points aided the prediction of a total of 13,277 gene models, including $99 \operatorname{RxLR}(-$ like) effector, and 14 putative Crinkler genes. Comparative analysis identified common features in the predicted secretomes of different obligate biotrophic oomycetes, regardless of their phylogenetic distance. Their secretomes are generally smaller, compared to hemi-biotrophic and necrotrophic oomycete species. We observe a reduction in proteins involved in cell wall degradation, in Nep1-like proteins (NLPs), proteins with PAN/apple domains, and host translocated effectors. The genome of Pfs 1 will be instrumental in studying downy mildew virulence and for understanding the molecular adaptations by which new isolates break spinach resistance. 


\section{Introduction}

Phytopathogenic oomycetes are eukaryotic microbes that infect a large range of plant species. Due to their hyphal infection structures they appear fungal-like, however, taxonomically they belong to the Stramenopiles [2]. The most devastating phytopathogenic oomycetes are found within the orders of Albuginales, Peronosporales and Pythiales (Pythiaceae).

The highly radiated Peronosporales order contains species with different lifestyles. The most infamous species of this order are in the hemibiotrophic Phytophthora genus. Other species within the Peronosporales are the obligate biotrophic downy mildews that cause disease while keeping the plant alive. The relationships between downy mildews and Phytophthora species have long been unresolved [27]. Until recently, downy mildew species were underrepresented in studies addressing oomycete phylogeny. This is mainly because the obligate biotrophic nature of the species makes them hard to work with and they are, therefore, under-sampled compared to other oomycete phytopathogens.

The first phylogenetic trees based on morphological traits and single gene comparisons [6, 24] classified the downy mildews as a sister clade to the Phytophthora species within the order of Peronosporales. Recently published studies using multiple gene and full genome comparisons, including a number of downy mildew species, suggest that the downy mildews have multiple independent origins within the Phytophthora genus [19, 22, 27].

The downy mildew Peronospora effusa (previously known as $P$. farinosa forma specialis spinaciae, and here referred to as Pfs), is the most important pathogen of spinach. Pfs affects the leaves, severely damaging the harvestable parts of the spinach crop. Under favorable environmental conditions, Pfs infection can progress rapidly resulting in abundant sporulation within a week post inoculation that is visible as a thick grey 'furry layer' of sporangiophores producing abundant asexual spores [30] Preventing spread of this pathogen is difficult, since only a few fungicides are effective in chemical control [116]. As a result, the disease can cause severe losses in this popular crop, and infected fields 
often completely lose their market value.

During infection, hyphae of $P f s$ grow intercellularly through the tissue and locally breach through cell walls to allow the formation of haustoria [117]. These invaginating feeding structures form a platform for the intimate interaction between plant and pathogen cells, and function as a site for the exchange of nutrients, signals and proteins. Oomycetes deliver proteins into plant cells to alter host immunity [118], thereby escaping and suppressing plant immune responses [119]. These and other molecules are secreted by pathogens to promote the establishment and maintenance of a successful infection in the host are called effectors. Effector proteins can either be functional outside the plant cells (apoplastic effectors) or inside plant cells (host-translocated effectors). Two types of host translocated are known in oomycetes; the RXLR and crinkler (CRN) effectors. They are characterized by the presence of a signal peptide, a conserved domain at the $\mathrm{N}$-terminus and a variable $\mathrm{C}$-terminal part which is responsible for the function of the effector in the cell [56, 120, 121].

Here we describe the sequencing of genomic DNA obtained from Pfs spores collected from infected spinach plants using a combination of Illumina and PacBio sequencing. Sequencing of obligate biotrophic species is complicated as the spore washes of infected plant leaves contain many other microorganisms. Bioinformatic filtering on taxonomy using the recently developed Contig Annotation Tool CAT [122] was deployed to remove the majority of contaminating sequences. The obtained assembly of race Pfs1 was used to predict genes and compare its proteome, in particular its secretome, with that of other oomycete taxa. We show that the secretomes of obligate biotrophic oomycetes are functionally more similar to each other than to that of more closely related species with a different lifestyle. 


\section{Materials and Method Downy mildew infection}

Peronospora effusa race 1 (Pfs1) was provided by the Dutch breeding company Rijk Zwaan Breeding BV in 2014. As Pfs 1 is an obligate biotrophic maintenance was done on Spinacia oleracea Viroflay plants. Seeds were sown on soil, stratified for two days at $4{ }^{\circ} \mathrm{C}$ and grown under long day condition for two weeks ( $16 \mathrm{~h}$ light, $70 \%$ humidity, $21^{\circ} \mathrm{C}$ ). sporangiophores were washed off infected plant material in $50 \mathrm{ml}$ Falcon tubes. The solution was filtered through miracloth and the spore concentration was checked under the microscope. Four-day-old Spinacia oleracea Viroflay plants were infected with Pfs by spraying a spore solution (70 spores/ ul) in tap water. Seven days post inoculation, Pfs sporangiospores were collected from heavily-infected spinach leaves with tap water, using a soft brush to prevent plant and soil contamination and used for DNA isolation and genome sequencing.

\section{DNA isolation and genome sequencing}

The sporangiospores were freeze-dried, ground and dissolved in CTAB (Cetyltrimethyl ammonium bromide) extraction buffer, lysed for 30 minutes at $65^{\circ} \mathrm{C}$, followed by a phenol-chloroform/isoamyl-alcohol, and chloroform/isoamyl-alcohol extraction. DNA was precipitated from the aqueous phase with $\mathrm{NaOAc}$ and ice-cold isopropanol. The precipitate was collected by centrifugation, and the resulting pellet washed with ice cold $70 \%$ ethanol. DNA was further purified using a QIAGEN Genomic-tip 20/G, following the standard protocol provided by the manufacturer. DNA was quantified using a Qubit HS dsDNA assay (Thermo Fisher Scientific) and sheared using the Covaris S220 ultrasonicator set to $550 \mathrm{bp}$. The sequencing library was constructed with the Illumina TruSeq DNA PCRFree kit. Fragment size distribution in the library was determined before and after the library preparation using the Agilent Bioanalyzer 2100 with HS-DNA chip (Agilent Technologies). The library was sequenced on an Illumina Nextseq machine in high output mode with a 550 bp genomic insert paired end $150 \mathrm{bp}$ reads. Illumina reads with low quality ends were trimmed $(Q<36)$ using prinseq-lite [123]. 
For PacBio sequencing the input DNA was amplified by WGA (Whole Genome Amplification) using the Illustra GenomiPhi V2 DNA Amplification (GE Healthcare). The sequencing library for PacBio was constructed according to the manufacturer protocol. The resulting library was sequenced on 24 SMRT cells (P6 polymerase and C4 chemistry) using the RSII sequencer (KeyGene N.V., Wageningen). The obtained PacBio reads were error-corrected using the FALCON pipeline [124] with the standard settings using the SMRT Portal that is part of the SMRT analysis software package version 2.3.0 from PacBio [125]. The analysis software package was installed according to the installation instructions on an Amazon WebService (AWS) cloud-based computer and operated via its build in GUI.

\section{Taxonomic classification of long reads}

The taxonomic origin of each error corrected PacBio read was determined using the CAT (Contig Annotation Tool) pipeline version 1.0 with default parameters [122]. To do this, CAT first identifies open reading frames (ORFs) on the long sequences or contigs using Prodigal [126] and queries them against the NCBI non-redundant (nr) protein database (retrieved November 2016) using DIAMOND [127]. A benchmarked weighting scheme is then applied that allows the contig to be classified with high precision [122].

\section{Genome assembly and identification of repeats}

A pre-assembly was made using taxonomically filtered and corrected PacBio sequences and $60 \%$ of the lllumina reads using SPAdes version 3.5.0 [128] The error-corrected PacBio reads were used as long reads in the assembly, SPAdes was set to use $k$-mer lengths of: $21,33,55,77,99$, 127 for the assembly and the --careful option was used to minimize the number of mismatches in the final contigs. The contigs derived from the pre-assembly were filtered using the CAT tool (see above), and sequences that were designated as bacterial or non-stramenopile eukaryotes were 
collected. The entire set of Illumina sequencing reads were aligned to the collection of removed sequences (annotated as bacterial and nonstramenopile) with Bowtie version 2.2.7 using default settings [129]. Illumina reads that aligned to these sequences were removed from the Illumina data set. The remaining Illumina reads (Illumina filtered), and PacBio sequences were re-assembled with SPAdes (same settings as the preassembly), which resulted in a final Pfs1 genome assembly. A custom repeat library for the Pfs 1 genome assembly was generated with RepeatModeler [130]. Repeat regions in the assembled Pfs1 genome were predicted using RepeatMasker 4.0.7 [130].

\section{Quality evaluation of the assembly}

$K$-mers of length 21 in the filtered Illumina data set were counted with Jellyfish count version 2.0 [131] with settings -C -m 21 -s 1000000000 followed by Jellyfish histo. The histogram was plotted with GenomeScope [132] to produce a graphical output and an estimate of the genome size. The coverage of the genome by PacBio sequences was determined by aligning the unfiltered error-corrected PacBio reads to the Pfs1 genome assembly using BWA-mem [133] and selected $-x$ pacbio option. The BBmap pileup [134] script was used to determine the percentage covered bases by PacBio reads in the final assembly of the Pfs 1 .

The GC-content per contig larger than $1 \mathrm{~kb}$ was calculated using a Perl script [135]83]. GC density plots were generated in Rstudio version 1.0.143 using GGplot version 3.1 [136]. For comparison, the same analysis was done on a selection of other publicly available oomycete assemblies; Hyaloperonospora (H.) arabidopsidis [59], Peronospora (P.) belbahrii [137], Phytophthora (Ph.) infestans [58], Bremia (B.) lactucae [61], Phytophthora parasitica [138], Phytophthora ramorum (Pr102) [138], Phytophthora sojae [138], Peronospora tabacina (968-S26) [60] and Plasmopara (PI.) viticola [22].

Kaiju [139] was used to analyze the taxonomic origin by mapping reads to the NCBI nr nucleotide database (November 2017). The input for Kaiju was generated using ART [140] set at 20x coverage with 150 
bp Illumina to create artificial sequencing reads from the various FASTA assembly files of the genomes of different oomycetes.

Genome completeness and gene duplications were analyzed with BUSCO version 3 [141] with default settings using the protists Ensembl database (May 2018).

\section{RNA sequencing and gene model prediction}

RNA of Pfs1 at different stages during the infection was isolated and sequenced to aid gene model prediction. Infected leaves and cotyledons were harvested every day from three days post infection (dpi) until sporulation (7 dpi). Besides these infected leaves, spores were harvested, and a subset of these spores were placed in a petri dish with water and incubated overnight at $16^{\circ} \mathrm{C}$ to allow them to germinate. RNA was isolated using the RNeasy Plant Mini Kit from Qiagen, and the RNA was analyzed using the Agilent 2100 bioanalyzer to determine the RNA quality and integrity. The RNA-sequencing libraries were made with the Illumina TruSeq Stranded mRNA LT kit. Paired-end 150 bp reads were obtained from the different samples with the Illumina Nextseq 500 machine on high output mode. RNA-seq reads from all the samples were pooled, aligned to the Pfs 1 assembly using Tophat [142], and used as input for gene model prediction using BRAKER1 [143] The obtained gene models for the Pfs1 genome together with the RNA-seq alignment result, the repeat models, and results obtained from a BLASTp search to the nr NCBI database (January 2017), were loaded into a locally-installed WebApollo [144] instance. Gene models on the 100 largest contigs of the genome were manually curated and all gene models were exported from WebApollo for further use.

\section{Gene annotation and the identification of functional domains}

Bedtools intersect version 2.27 was used to determine the overlap between Pfs1 gene models and annotated repeat elements in the genome. Gene models that had more than $20 \%$ overlap with a region marked as a repeat- 
containing gene. ANNIE [145] was used to annotate proteins on the Pfs1 genome based on Pfam domains [146] and homologous sequences in the NCBI-Swissprot database (accessed Augustus 2017). Sequences that were annotated as transposons by ANNIE were removed from the gene set. SignalP 4.1 [147] was used to predict the presence and location of a signal peptide, the D-cutoff for noTM and TM networks were set at 0.34 to increase sensitivity [148]. TMHMM version 2 [149] was used to predict the presence of transmembrane helices in the proteins of Pfs1. To identify proteins that possess one or more WY domains an HMM model made by Win et al. [150] was used. Protein sequences that possessed a WY domain were extracted and realigned. This alignment was used to construct a new HMM model using HMMER version 3.2.1 [151] and queried again against all protein models in the Pfs genome to obtain the full set of WY domains containing proteins.

\section{Effector identification}

Putative effectors residing on the genome of Pfs1 were identified with a custom- made pipeline [152] constructed using the Perl [153] scripting language. Secreted proteins were screened for the occurrence of known translocation domains within the first 100 amino acids after the signal peptide. Proteins with a canonical RxLR, or a degenerative RxLR ( $x x L R$ or RxLx) combined with either an EER-like or a WY domain or both where considered putative RXLR effectors. A degenerative EER domain was allowed to vary from the canonical EER by at most one position.

Proteins with a canonical LFLAK motif or a degenerative LFLAK and HVL motif in the first 100 amino acids of the protein sequence. A HMMer profile was constructed based on the LFLAK or HVL containing proteins. This HMMer profile was used to identify Crinkler effector candidates lacking the LFLAK or HVL motif.

Proteins with an additional transmembrane domain or a C-terminal ER retention signal $(\mathrm{H} / \mathrm{KDEL})$ were removed. WY domains were identified using hmmsearch version 3.1b2 [154] with the published Phytophthora HMM model (see above) [77]. Pfs WY-motif containing 
protein sequences were realigned and used to construct a Pfs specific WY HMM model using hmmbuild version 3.1b2 [154]. Based on the Pfs specific HMM model WY-motif containing Pfs proteins were determined.

The effector prediction for the comparative analysis was done in a similar fashion, except the published Phytophthora HMM model for RxLR prediction and a published model for CRN prediction was used [155]. The prediction of effectors using the same model in each species enabled the comparison.

\section{Comparative gene distance analysis}

Based on the gene locations encoded in the GFF file the $3^{\prime}$ and 5 intergenic distances between genes on contigs were calculated as a measure of local gene density. When a gene is located next to beginning or end of a contig, the distance was taken from the start or end of the gene to the end of the contig. Putative high confidence RXLR effector sequences that encode for proteins with either an exact canonical $R x L R$ motif or an RxLR-like motif in combination with one or more WY-motifs were selected for the comparison (66 in total). Distances were visualized using a heat map constructed with the GGPlot geom_hex function [136]. Statistical significance was determined using the Wilcoxon signed-rank test [156].

\section{Comparative secretomics}

The predicted proteomes of eighteen plant pathogenic oomycetes were obtained from Ensembl and NCBI (Table S1). Proteins in the collected proteomes that have a predicted secretion signal [147] (SignalP v.4.1, D-cutoff for SignalP-noTM and TM networks $=0.34$ [148]), no additional transmembrane domain (TMHMM 2.0 [149]) or C-terminal K/HDEL domain were considered secreted. Functional annotations of the secreted proteins were predicted using InterProScan [157] and the CAZymes database [158] using the dbCAN2 meta server [159]. 


\section{Phylogenetic analysis}

The phylogenetic relationships between the proteomes of the studied species were inferred using Orthofinder [160]. Orthofinder first identifies 'orthogroups' of proteins that descended from a single ancestral protein. Next it determines pairwise orthologs between each pair of species. Orthogroups with only one protein of each species were used to make gene trees using MAFFT [161]. The species tree was inferred from the gene trees using the distance algorithms of FastMe [162] and visualized using EvolView v2 [163].

\section{Principal Component Analysis}

The total number of InterPro and CAZymes domain per species was summarized in a counts table. For each domain the number was divided by the total number of domains for that species. The normalized matrix has been loaded into Phyloseq version 1.22.3 [164] with $R$ version 3.4.4 [165] in RStudio [166]. A PCA plot has been made with the Phyloseq ordinate function on euclidean distance. The PCA plot has been made with the GGPlot R package [136]. The biplot has been generated with the standard prcomp function in $\mathrm{R}$ with the same normalized matrix. Figures were optimized using Adobe Photoshop 2017.01.1.

\section{Permutational analysis of variance (PERMANOVA)}

A PERMANOVA using distance matrices was used to statistically test whether there is a difference between the clades based on their CAZymes and InterPro domains. PERMANOVA is a non-parametric method for multivariate analysis of variance using permutations. The data has been double root transformed with the vegdist function from the R-package vegan version 2.5-3 [167]. After the transformation the PERMANOVA has been calculated with the adonis function from the Vegan package. A total number of 999 permutations have been made to retrieve a representative permutation result. 


\section{Enrichment analysis}

A chi-square test with Bonferroni correction was used to identify underand over-represented Pfam domains in each group (Hyaloperonospora/ Peronospora, Plasmopara, Albugo) compared to Phytophthora. The actual range was the sum of the proteins that have a given domain. The expected range was the fraction of proteins with a given domain that is expected to belong to a species cluster giving the overall ratio of Pfam domains between species clusters.

\section{Results}

An early race 1 isolate, Pfs 1 , of Peronospora effusa was used to create a reference genome as it predates resistance breeding in spinach and its infection is effectively stopped by all spinach resistance genes known to date. Race 1 was first identified in 1824 [168]. Since downy mildews cannot be grown axenically we isolated asexual sporangiospores by carefully washing highly-infected leaves of the universally susceptible cultivar Viroflay. Genomic DNA was isolated from freeze-dried spores and used to construct libraries for $\mathrm{PacBio}$ and Illumina sequencing, resulting in 1.09 million PacBio reads with a N50 of 9,253 bp, and 535 million Illumina reads of $150 \mathrm{bp}$. The paired-end Illumina reads were used for a trial assembly using Velvet. Inspection of the draft assembly showed that many contigs were of bacterial instead of oomycete origin. This is likely caused by contamination of the isolated Pfs spores with other microorganisms that reside on infected leaves and that are collected in the wash-offs. We, therefore, decided to treat the sequences as a metagenome and bioinformatically filter the sequences and corresponding reads.

\section{Taxonomic filtering}

To filter out the sequences that could be classified as contaminants we deployed CAT [122] on long reads and contigs derived from assemblies. Details on the CAT method are described in the materials and methods section. In short, CAT utilizes the combined taxonomic annotations of multiple individual ORFs found on each sequence to determine its likely 
taxonomic origin. This allows for a robust taxon classification that is based on multiple hits, rather than a single best hit. An example of the CAT taxonomic classification for two of our sequences (contigs) is visualized in Fig.1.

A
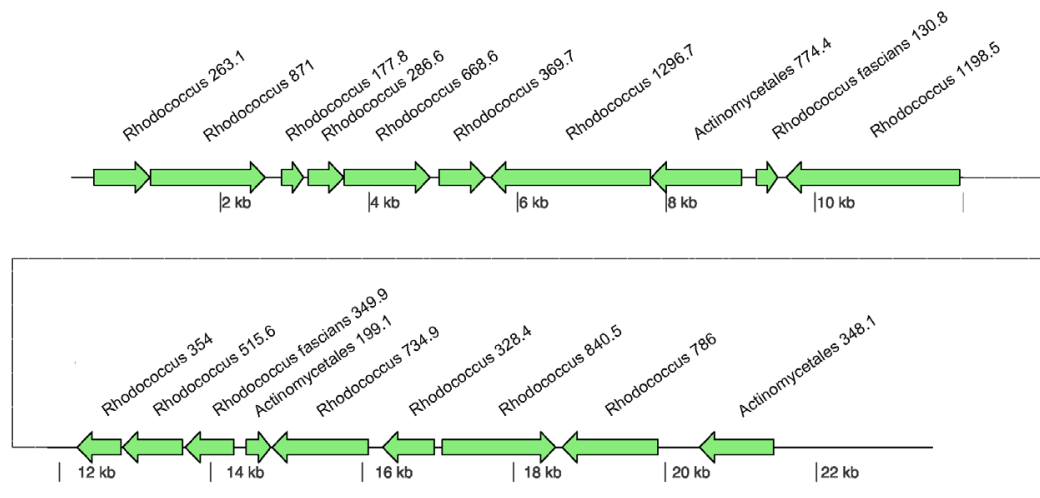

B
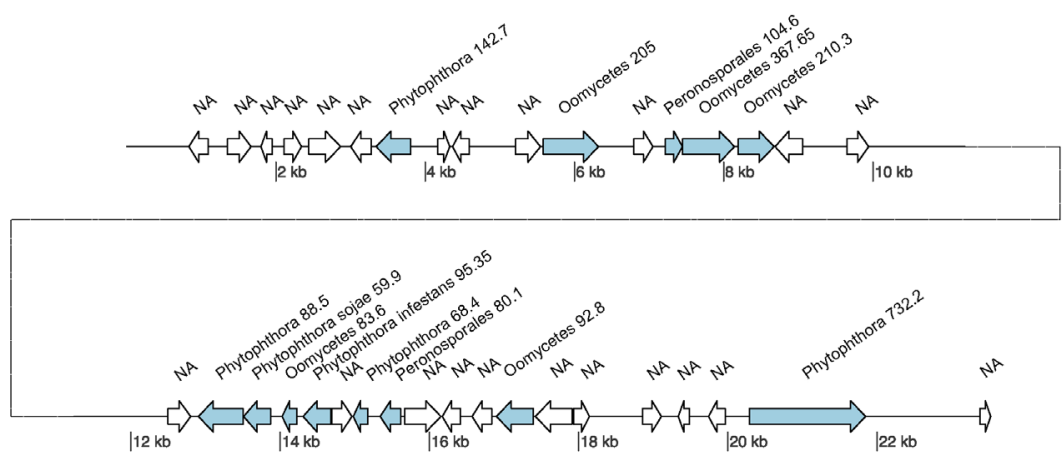

Figure 1. Taxonomic classification by CAT.

Two contigs are depicted and per ORF a single top hit is shown. (A) Contig from the pre-assembly assigned by the CAT tool as bacterial, ORFs of bacterial origin are colored green, and ORF with no hits to the database are colored white. On this contig most ORFs had a highest blast hit with Rhodococcus species. The $\Sigma$ Bmax for this contig is 10982. and the highest $\Sigma$ Btaxon is for the Rhodococcus genus at 9660, which is well above the cutoff of 5491 ( $\left.\Sigma \mathrm{Bmax}{ }^{*} 0.5\right)$. The taxonomic origin of this contig was therefore assigned to the genus Rhodococcus, and as a consequence this contig was regarded as nonPfs and removed. (B) Contig from the pre-assembly assigned by the CAT tool as an oomycete contig. On this contig all ORFs have a best hit to an oomycete species, and the $\Sigma$ Bmax is 2328. In fact, most ORFs have a best hit to species in the Phytophthora

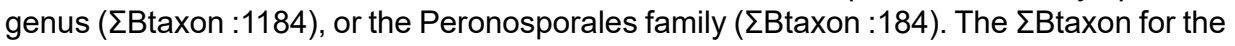
Phytophthora genus is above the cutoff at $1164\left(\Sigma \mathrm{Bmax}{ }^{*} 0.5\right)$ thus assigning this contig to the Phytophthora genus, and consequently this contig is maintained for the Pfs genome assembly. 
CAT was first used on the long PacBio reads. As these reads contain about $15 \%$ base call errors on average, they were first error-corrected using the FALCON pipeline. The FALCON pipeline fixes long PacBio reads by mapping short reads obtained in the same runs. The resulting 466,225 PacBio reads had a total length of $1,003 \mathrm{Mb}$ with a N50 of 3,325 bp and were subsequently assigned a taxonomic classification using CAT. PacBio reads that were classified as prokaryotic, or non-stramenopile eukaryotic (e.g. Fungi) were removed, whereas reads with the assigned taxonomy "stramenopiles" or "unknown" were retained. This resulted in a cleaned set of 232,846 PacBio reads with a total length of $522 \mathrm{Mb}$ with a N50 of 3,458 bp that was used for a hybrid pre-assembly. In order to evaluate the effectiveness of the CAT tool in removing contaminating genomic sequences we analyzed the GC-content of the reads. The corrected PacBio reads showed two distinct peaks (Fig. 2a), whereas oomycete genomes have a GC band-width around $50 \%$, as shown in Fig. S1a for the contigs of the Phytophthora infestans genome [58]. After CAT filtering a single peak remained with a narrow GC-content distribution around $\sim 48 \%$, demonstrating that the tool, that does not take into account GC-content but uses a weighting scheme based on protein sequence similarity, was effective in removing contaminating sequences (Fig. 2b).

\section{Hybrid assembly}

A hybrid pre-assembly was generated using the genome assembler SPAdes that can combine long PacBio with short Illumina reads. The input consisted of all corrected and filtered PacBio reads together with $60 \%$ randomly extracted Illumina reads (321 Million read, $96.3 \mathrm{~Gb}$, to decrease assembly run time and memory requirements). The preassembly consisted of 170,143 contigs with a total length of $176 \mathrm{Mb}$ and an N50 of 6,446 bp, of which only 21,690 contigs were larger than $1 \mathrm{~kb}$. CAT filtering was applied to the contigs of the pre-assembly, CAT marked 16,518 contigs consisting of $91.5 \mathrm{Mb}$ ( $52 \%$ of total assembled bases) as contaminant sequences. Next, Illumina reads were aligned to these and Illumina read-pairs of which at least one end aligned were removed from 
the data set. A final assembly was generated with the CAT-filtered PacBio and remaining 77.6 million Illumina reads, resulting in 8,635 scaffolds with a total length of $32.4 \mathrm{Mb}$. The assembly size corresponds with the estimate genome size of $36,2 \mathrm{Mb}$ that was determined based on $k$-mer count frequency (Table 1) in the filtered Illumina reads.

\section{Filtering results}

The effect of filtering with CAT on the pre-assembly is well visualized by plotting the GC-content of the contigs (Fig. 2c), similar as for the PacBio reads. In the pre-assembly many contigs with a GC-percentage deviating from the $40-55 \%$ range are present, indicating that it contains many contaminating sequences. After filtering, the final assembly shows one major peak of the expected GC-content at $\sim 48 \%$, with a minor shoulder of slightly higher GC-content (Fig. 2d). 

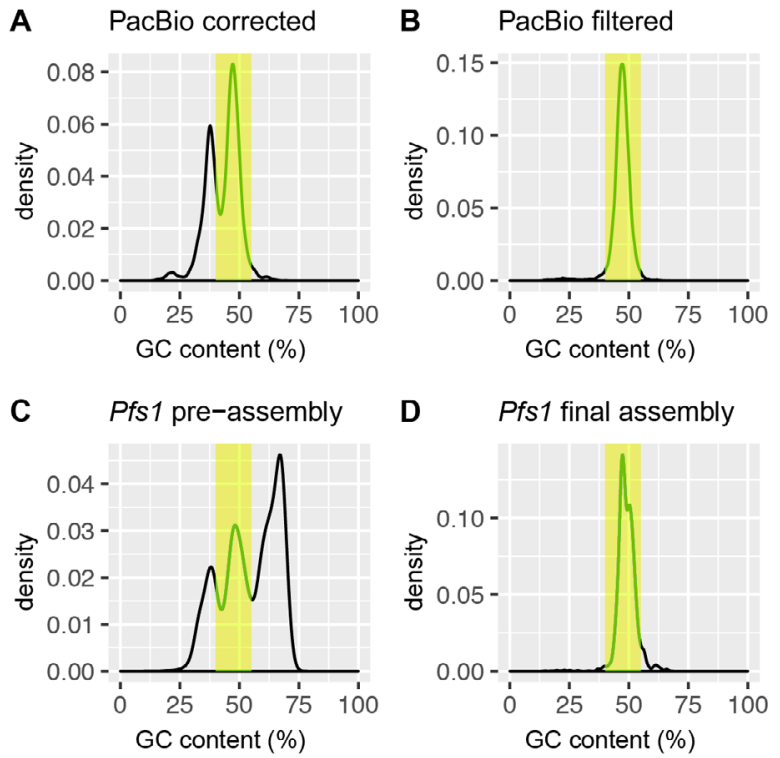

Figure 2. Density plot of the GC values of PacBio reads and assembly before and after CAT filtering of sequences.

The yellow bar indicates the region between 40 and $55 \%$ GC, based on reads $>1 \mathrm{~kb}$. (A) PacBio reads before CAT-filtering show a bimodal distribution with a presumed peak of contaminating sequences with a GC content of $\sim 40 \%$. (B) PacBio reads after CAT-filtering show a distribution consisting of a single peak with a GC content around $\sim 46 \%$. (C). GC content of the Pfs 1 contigs from the pre-assembly before filtering shows additional peaks at around 30 and $60 \mathrm{GC} \%$, indicating that there are many contaminant contigs. (D) GC content of the Pfs1 contigs after filtering of the reads with the CAT tool shows that the additional peaks are no longer present and have thus been successfully filtered out.

To assess the effectiveness of the taxonomic filtering we used Kaiju [139] as a complementary tool. Kaiju is typically used for taxonomic classification of sequencing reads in metagenome analysis but here we used it to determine the effect of taxonomic filtering by CAT. For this, genome assemblies of Pfs 1 and other oomycetes were divided into artificial short reads. The taxonomic distributions generated by Kaiju provide a clear picture of the removal of contaminating sequences from the Pfs 1 genome data (Fig. 3). Whereas the pre-assembly mostly contained artificial reads with an assigned bacterial taxonomy, this was reduced to $14 \%$ in the final assembly. The percentage of $>80 \%$ of oomycete-assigned reads in the Pfs 1 final assembly is similar to what we observe for the high-quality 
genome assemblies of $P$. infestans and $P$. sojae, pathogens that can be grown axenically, i.e. free of contaminating other microbes (Fig. 3).

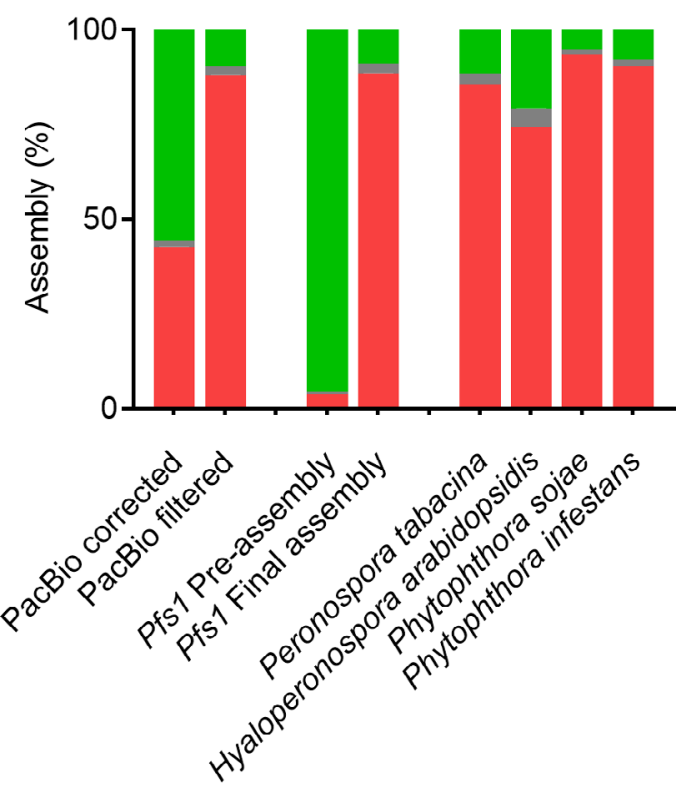

Figure 3. Taxonomic classification of reads in assemblies of different oomycetes.

Kaiju bar plot showing the percentage of reads assigned to three taxonomical classes; Oomycetes, Fungi and Bacteria and other non-oomycetes. In error corrected PacBio reads $42.64 \%$ are assigned to oomycetes, after filtering with CAT $88.09 \%$ of the reads are assigned to oomycetes. For the pre-assembly $(96.3 \mathrm{~Gb})$, only $5 \%$ of the artificial reads is assigned to oomycetes. For the Pfs 1 final assembly $(32.4 \mathrm{Mb}), 88.6 \%$ of the reads are assigned to oomycetes. This is comparable to other oomycetes that can be axenically grown on plates, indicating that the remaining non-oomycete-assigned sequences are most likely a result of an incorrect classification in the database.

\section{Genome statistics}

To assess the quality of the assembly we re-aligned the Illumina reads to the contigs and found a large variation in coverage between the contigs smaller than $1 \mathrm{~kb}$ and the larger contigs, suggesting that these small contigs contain a high number of repeats or assembly errors. In addition, the CAT pipeline depends on classification of individual ORFs on contigs, so it's accuracy may be expected to improve with contig length. Therefore, several small contigs could possibly be derived from microbes other than Pfs. Removing contigs smaller than $1 \mathrm{~kb}$ (5027 contigs) resulted in a small 
reduction of $1.9 \mathrm{Mb}$ in genome length, slightly reducing the assembly size to $30.5 \mathrm{Mb}$, but resulting in a $58 \%$ reduction in the number of contigs. The remaining 3608 contigs, larger than $1 \mathrm{~kb}$, had an N50 of 36,273 bp. The statistics of the size-filtered assembly are further detailed in Table 1.

Table 1. Summary of statistics for the hybrid assembly of the Pfs1 genome.

\begin{tabular}{|l|l|l|}
\cline { 2 - 3 } \multicolumn{1}{c|}{} & Pfs1 final & Pfs1 size-filtered \\
\hline Assembly size & $32.40 \mathrm{Mb}$ & $30.48 \mathrm{Mb}$ \\
\hline GC content & $47.75 \%$ & $47.80 \%$ \\
\hline Longest scaffold & $310.10 \mathrm{~kb}$ & $310.10 \mathrm{~kb}$ \\
\hline Repeat size & $6.93 \mathrm{Mb}$ & $6.38 \mathrm{Mb}$ \\
\hline \# Contigs & 8,635 & 3,608 \\
\hline N50 & $32,837 \mathrm{bp}$ & $36,273 \mathrm{bp}$ \\
\hline \# Gene models & 13,227 & 12,630 \\
\hline
\end{tabular}

\begin{tabular}{|l|l|}
\cline { 2 - 2 } \multicolumn{1}{c|}{} & k-mer estimation \\
\hline Assembly size & $36.18 \mathrm{Mb}$ \\
\hline Repeat size & $8.76 \mathrm{Mb}$ \\
\hline Read Error Rate & $1.04 \%$ \\
\hline
\end{tabular}

Data is provided for the final assembly ( $P f s 1$ final) and size-filtered assembly omitting the contigs smaller than $1 \mathrm{~kb}$ (Pfs1 filtered). In addition, genome information based on $k$-mer counting of the Illumina reads is provided, giving an estimate for the predicted genome size and repeat content.

To assess the gene space completeness of our assembly in comparison to other oomycete genomes we used BUSCO that identifies single core orthologs that are conserved in a certain lineage. Here, we used the protist Ensembl database as the protist lineage encompasses the oomycetes and other Stramenopila. According to the BUSCO analysis the gene space in our final assembly is $88.9 \%$ complete with only $0.5 \%$ fragmented genes and $0.5 \%$ duplicates. This gene space completeness score is similar to that of other downy mildew genomes, but slightly lower than of genomes of Phytophthora species (Table S2). Furthermore, the low number of duplicates suggests that there is a low incidence of 
erroneous assembly of haplotypes, suggesting that the obtained Pfs assembly represents most of the single-copy gene space of the Pfs genome [141].

\section{Repeat content}

In addition to a genome size estimate, the $k$-mer analysis estimated a repeat content of $\sim 8.8 \mathrm{Mb}$. This is slightly higher than the observed repeat content in the final assembly of $\sim 6.9 \mathrm{Mb}(\sim 6.4 \mathrm{Mb}$ in the sizefiltered assembly) (Table 1). The difference between the estimated repeat size and the repeat content in the assembly $(1.87 \mathrm{Mb})$ is most likely caused by long repetitive elements that are hard to assemble. Repeatmasker [130] identified a total of 13,089 repeat elements of which most are part of the Gypsy and Copia superfamily. We also identified 562 LINEs (Long interspersed nuclear elements) and only 16 SINE (short interspersed nuclear elements), which belong to the class I transposon (retrotransposons). Other repeat elements consisted of 2297 simple repeats, 298 Low complexity regions, 391 different types of DNA transposons (Table 2), and several (278) other minor repeat types; full details can be found in Table S3.

Table 2. Total number and size of major repeat types identified in the Pfs 1 genome assembly.

\begin{tabular}{|l|l|l|l|}
\hline Repeat type & Count & \% of total count & Total length (bp) \\
\hline LTR & 9247 & 70,65 & 6532069 \\
\hline LINE & 562 & 4,29 & 201127 \\
\hline Simple repeat & 2297 & 17,55 & 97983 \\
\hline DNA repeats/TE & 391 & 2,99 & 46677 \\
\hline Rolling Circle TE & 97 & 0,74 & 26123 \\
\hline
\end{tabular}

The percentage of total count is based on the total number of repeat types identified in the assembly which can be found in Table S3. 
When we compare the genome assembly size of $P f s(30.5 \mathrm{Mb})$ to other sequenced oomycete genomes such as those of Ph. infestans (240 $\mathrm{Mb}), H$. arabidopsidis (100 Mb), Pl. halstedii $(75.3 \mathrm{Mb}$ ) or the relatively small genome of $P$. tabacina $(63.1 \mathrm{Mb})$, Pfs has a strikingly compact genome (Table S4). The repeat content $(21 \%)$ is also low compared to that of other oomycetes, e.g. Ph. infestans (74\%), H. arabidopsidis (43\%), PI. halstedii (39\% Mbp) and more comparable to P. tabacina (24\%).

\section{Pfs gene prediction RNA sequencing}

Gene prediction is greatly aided by transcript sequence information. We, therefore, isolated and sequenced mRNA from Pfs spores and Pfsinfected spinach leaves at several time points during the infection. For this, leaves were harvested daily starting from 3 days post inoculation (dpi) until 7 dpi when sporulation was observed. In addition, mRNA was also isolated from sporangiospores and germlings grown from spores that were incubated in water overnight. The 7 different samples ensure a broad sampling of transcripts to facilitate gene identification. Illumina transcript sequences (659 million) were aligned to the assembled Pfs genome which resulted in $\sim 100$ million aligned read pairs. Most of the other reads map to the spinach genome but were not further analyzed.

\section{Predicted proteins}

The aligned transcript read pairs served as input for the BRAKER1 [143] pipeline to generate a Pfs specific training set for gene model prediction. This was then used to predict 13227 gene models on the final assembly. The corresponding protein models were annotated using ANNIE [145] and provided putative annotations for 7297 Pfs proteins (Table S5). We found that 12630 protein models reside on contigs larger than $1 \mathrm{~kb}$ and are thus contained in the size-filtered assembly. In addition, we found that 2983 gene models had $20 \%$ or more overlap with a repeat that was identified by RepeatMasker [130], another 952 protein models were annotated by ANNIE as transposable elements. When analyzing protein 
models that reside on small contigs $(<1 \mathrm{~kb})$ we observe that most of them $(61 \%)$ have a significant overlap with a repeat region and are marked by ANNIE as transposons. The number of gene models found in the assembly of Pfs1 is strikingly low in comparison to that in Ph. infestans $(17,792), H$. arabidopsidis $(14,321), P$. halstedii $(15,469)$ and more similar to $P$. tabacina $(11,310)$.

\section{Secretome and host-translocated effectors}

For the identification of the Pfs secretome as well as of candidate hosttranslocated RxLR and Crinkler effectors we choose to start with the proteins encoded by the initial 13,227 gene set. This reduced the risk of missing effectors that are encoded on smaller contigs $(<1 \mathrm{~kb})$. SignalP [147] prediction identified 783 proteins with a N-terminal signal peptide. Of these, 231 were found to have an additional transmembrane domain (as determined by TMHMM [149] analysis) leaving 557 proteins. In addition, five of these carried a C-terminal H/KDEL motif that functions as an ER retention signal. The resulting set of 552 secreted proteins, $\sim 4 \%$ of the Pfs1 proteome, was used for secretome comparison.

Previous research showed that some effectors of the lettuce downy mildew Bremia lactucae have a single transmembrane domain in addition to the signal peptide [169]. Therefore, we chose to predict the host-translocated effectors not only from the secretome but also from the set of proteins with a signal peptide and an additional transmembrane domain. A total of 99 putative RxLR or RXLR-like proteins and 14 putative Crinkler effectors were identified (Table S6). Ten putative RxLR effector proteins were found to have a single transmembrane domain. Also, five putative RxLR effectors were found on contigs smaller than $1 \mathrm{~kb}$ (Table S6). Of the 99 RxLR effectors, 64 had a canonical RxLR domain, while 35 had a degenerative RxLR domain combined with an EER-like and/ or WY domain [170]. The number of host-translocated effectors in Pfs is significantly smaller compared to that of Phytophthora species (eg. 563 RxLR and 385 effector genes in the genomes of $P$. infestans [58] and $P$. sojae [171] respectively). Crinkler effectors are charaterized by 
the N-terminal five amino acid "LFLAK" domain [56] Five of the identified putative Crinkler effectors had a canonical LFLAK domain. The others had a degenerative LFLAK combined with an HVL domain or were identified using the custom made Crinkler HMM.

\section{Genomic distribution of effectors}

It was previously described for the potato late blight pathogen $P$. infestans that effectors often reside in genomic regions with a relatively large repeat content compared the rest of genome [172]. To test this in Pfs, the distance between neighboring genes was measured to estimate the genomic context of the 13277 Pfs 1 genes in general and for 66 selected RxLR effector (canonical RxIR and degenerative RxLR with WY-motifs) genes specifically. To get a good overview of the intergenic distances we plotted the 3' and 5' values for all the genes in the Pfs1 genome on a $\log 10$ scaled heat map (Fig. 4). 


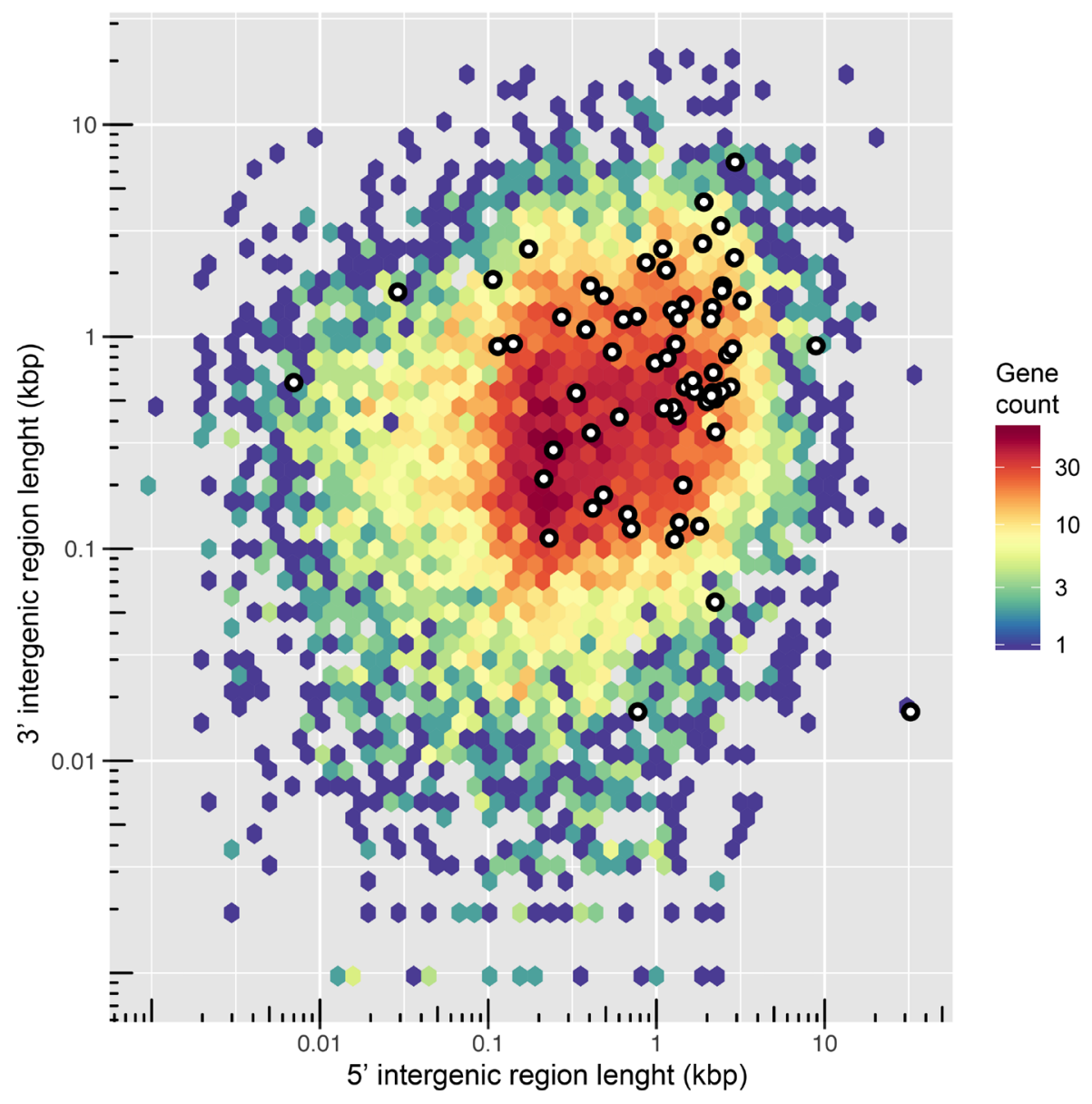

Figure 4. Genome spacing of predicted genes of Pfs1.

The distance between neighbouring genes was depicted by plotting the $5^{\prime}$ and $3^{\prime}$ intergenic distances (on a log10 scale) for each if the 13,227 predicted genes. The scale bar represents the number of genes in each bin, shown as a color-coded hexagonal heat map in which red indicates a gene dense and bleu a gene-poor region. The locations of putative Pfs effectors genes are indicated with white dots.

The genome of Pfs1 is highly gene dense and effectors show a modest but significant (Wilcoxon rank sum test, $p=1.914 \mathrm{e}^{-11}$ ) enrichment in the gene-spare regions of the genome (Fig. 4). The median 3' and 5' combined spacing for all genes is $925 \mathrm{bp}$, while for the selected effector genes it is $2976 \mathrm{bp}$. However, the difference in gene density between the effectors and core genes is not as strong as in the $P$. infestans two-speed genome [58]. 


\section{Comparative analysis of orthologs}

Eighteen phytopathogenic oomycete species, that represent a diverse taxonomic range and different lifestyles, were chosen for a comparative analysis with Pfs (Table 3). The objective of the comparison is to see whether the biotrophic lifestyle of downy mildew species, like Pfs, is reflected in the secretome. For the analysis, the secretome of Pfs was compared to that of closely related Phytophthora (hemibiotrophic), Plasmopara (biotrophic) and more distantly related Pythium (necrotrophic) and Albugo (biotrophic) species. First, the predicted proteins of each species were used to create a multigene phylogenetic tree to infer their taxonomic relationships using Orthofinder. In total, $86.9 \%(267,813)$ of all proteins were assigned to 14,484 orthogroups. Of those, 2383 had proteins from all species in the dataset of which 152 groups contained proteins corresponding to single copy proteins in each species. These single-copy orthologous proteins of each species were used to infer a Maximum-likelihood species tree (Fig. 5). 

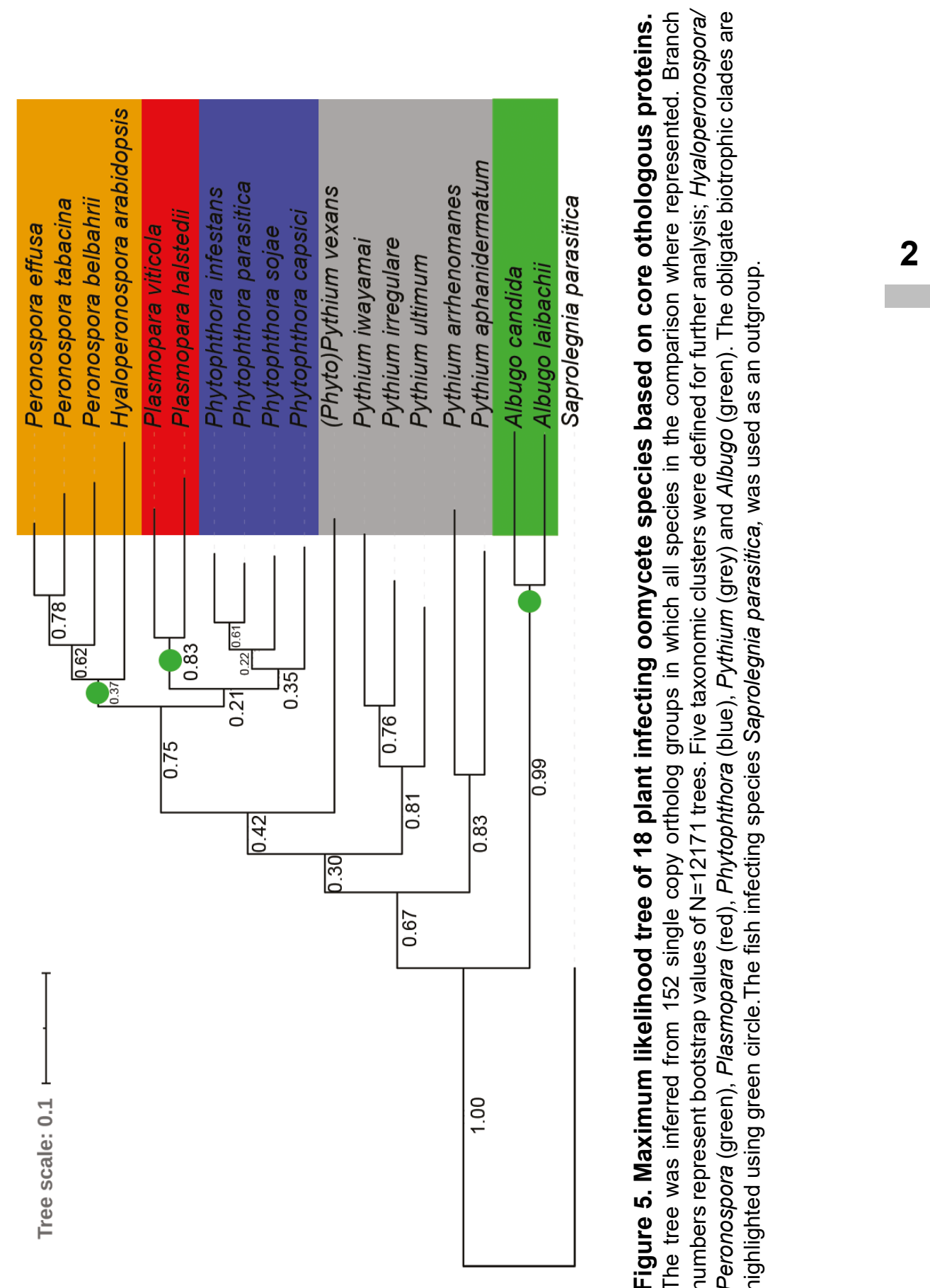

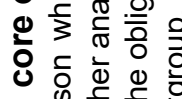

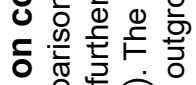

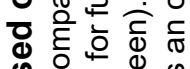

শิ

๑

ט.

¿

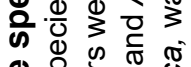

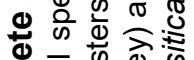

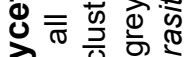

층 은 은

ठำ $\frac{1}{3}$ 을

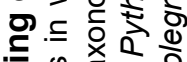

음

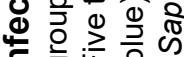

든

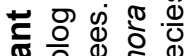

증 은

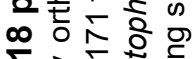

는 흥

- 은 I

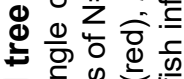

ช

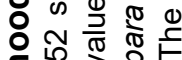

든 응 응

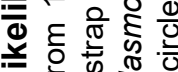

ह 을

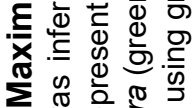

เ

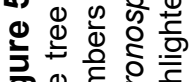

은 들 일 
The resulting tree shows that $P f s$ clusters with $H$. arabidopsidis (Hpa), P. tabacina (Pta) and P. belbahrii (Pbe). The closest relative of $P f s$, in this study, based on single-copy orthologs is the downy mildew of tobacco Pta, followed by the basil-infecting Pbe. Based on the tree, Hpa is more divergent from the former three downy mildew species within the Hyaloperonospora/Peronospora clade. The Plasmopara downy mildew species are in a different clade that is more closely related to the Phytophthora species used in this study. The separation between the Peronospora lineage and the Phytophthora/Plasmopara lineages is well supported with a bootstrap value of 0.75 . This clustering pattern is in line with the recent studies that suggest that the downy mildew species are not monophyletic within the Peronosporales [27, 173]. The Phytophthora species, although belonging to three different Phytophthora clades, are more closely related to each other than to the other species in this study. Phytopythium vexans appears as a sister group to the Phytophthora/ Peronospora lineage, which is in line with a recently published multi gene phylogeny [20]. The other five species of Pythium form two clusters, as previously observed [20]. The two Albugo species form a cluster that is separated from the other clades with maximum bootstrap support.

Based on the core ortholog protein tree, we grouped the species into five phylogenetically-related clades; Hyaloperonospora/ Peronospora, Plasmopara, Phytophthora, Pythium and Albugo for further analysis of the secretomes. Three of these clades only have obligate biotrophic species (Hyaloperonospora/Peronospora, Plasmopara and Albugo), whereas the Phytophthora cluster consists of hemi-biotrophs and Pythium cluster of necrotrophic species. (Phyto)Pythium vexans was included in the Pythium cluster. The fish-infecting oomycete Saprolegnia parasitica served as an outgroup for the phylogenetic tree and is not used for further comparison. 


\section{Secretome comparison}

For each species, the total number of proteins and the subset that is predicted to be secreted (signal peptide, no additional transmembrane domains, no ER retention signal) is shown in Table 3. Phytophthora species generally have a larger proteome than downy mildew species and secrete a larger percentage of the predicted proteins. The Phytophthora species in this study are predicted to secrete 1976 proteins on average, whereas the Plasmopara and Peronospora species secrete an average of 1461 and 703 proteins, respectively.

Table 3. Predicted secretomes of 18 oomycete species used in this study.

\begin{tabular}{|l|l|l|l|}
\hline & Predicted proteins & Secretome & $\%$ secreted \\
\hline P. effusa & 13227 & 552 & 4,2 \\
P. belbahrii & 9049 & 494 & 4,7 \\
H. arabidopsidis & 14321 & 999 & 7 \\
P. tabacina & 18447 & 798 & 4,3 \\
Pl. halstedii & 15498 & 1071 & 6,9 \\
Pl. viticola & 12201 & 1850 & 15,2 \\
Ph. infestans & 18138 & 1885 & 10,4 \\
Ph. parasitica & 27942 & 2250 & 8,1 \\
Ph. sojae & 26584 & 2337 & 8,8 \\
Ph.capsici & 19805 & 1433 & 7,2 \\
Py. arrhenomanes & 13805 & 913 & 6,6 \\
Py. aphanidermatum & 12312 & 928 & 7,5 \\
Py. irregulare & 13805 & 961 & 7 \\
Py. iawyamai & 15249 & 1067 & 7 \\
Py. vexans & 11958 & 863 & 7,2 \\
Py. ultimum & 15322 & 1071 & 7 \\
A. candida & 13310 & 888 & 6,8 \\
A. laibachii & 13804 & 679 & 4,9 \\
\hline
\end{tabular}

The total number of predicted proteins, those with a signal peptide (SP), proteins with SP but without additional transmembrane domains (TM), and the number of proteins with SP, no TM, and no C-terminal KDEL sequence are shown. In the final column the percentage of the proteome that is predicted to be secreted is highlighted. 


\section{Carbohydrate active enzymes and Pfam domains}

The secretome content was compared between species by looking at the carboydrate-active enzymes (CAZymes) and Pfam domains. CAZymes are, amongst others, involved in degrading and modifying plant cell walls, which is an important part of the infection process. The Pfam domain database represents a broad collection of protein families, including RxLR effectors, with diverse functions.

A total of 95 different CAZyme domains were found in the combined secretomes of the 18 oomycete species. The total number of CAZymes per species ranges from 35 in A. laibachii to 336 in $P$. sojae, and was lower in obligate biotrophic species (35 - 193) compared to Phytophthora species (197 - 336) (Table S7). A total of 1354 different Pfam domains were found in the combined secretomes of the oomycetes analyzed. The number of domains identified ranged from 304 in Al. candida to 1710 in Ph. parasitica. The total number as well as the relative number of Pfam domains in secretomes of obligate biotrophic species was lower in obligate biotrophic species compared to Phytophthora and Pythium (Table S8).

The presence and numbers of CAZyme and Pfam domains were compared between species using a Principal Component Analysis (PCA), a statistical reduction technique that determines what variables contribute most to the variation observed in a data set. We report the relative abundance of each CAZyme/Pfam domain to the total number of secreted Pfam/CAZyme domains per species, to account for the large variation in absolute numbers of proteins between the species (Fig. 6). PCAs based on the absolute numbers can be found in Fig. S2 and S3, which shows a similar pattern. The species clusters as depicted in Fig. 6 were confirmed using a PERMANOVA $(p<0.001)$. 

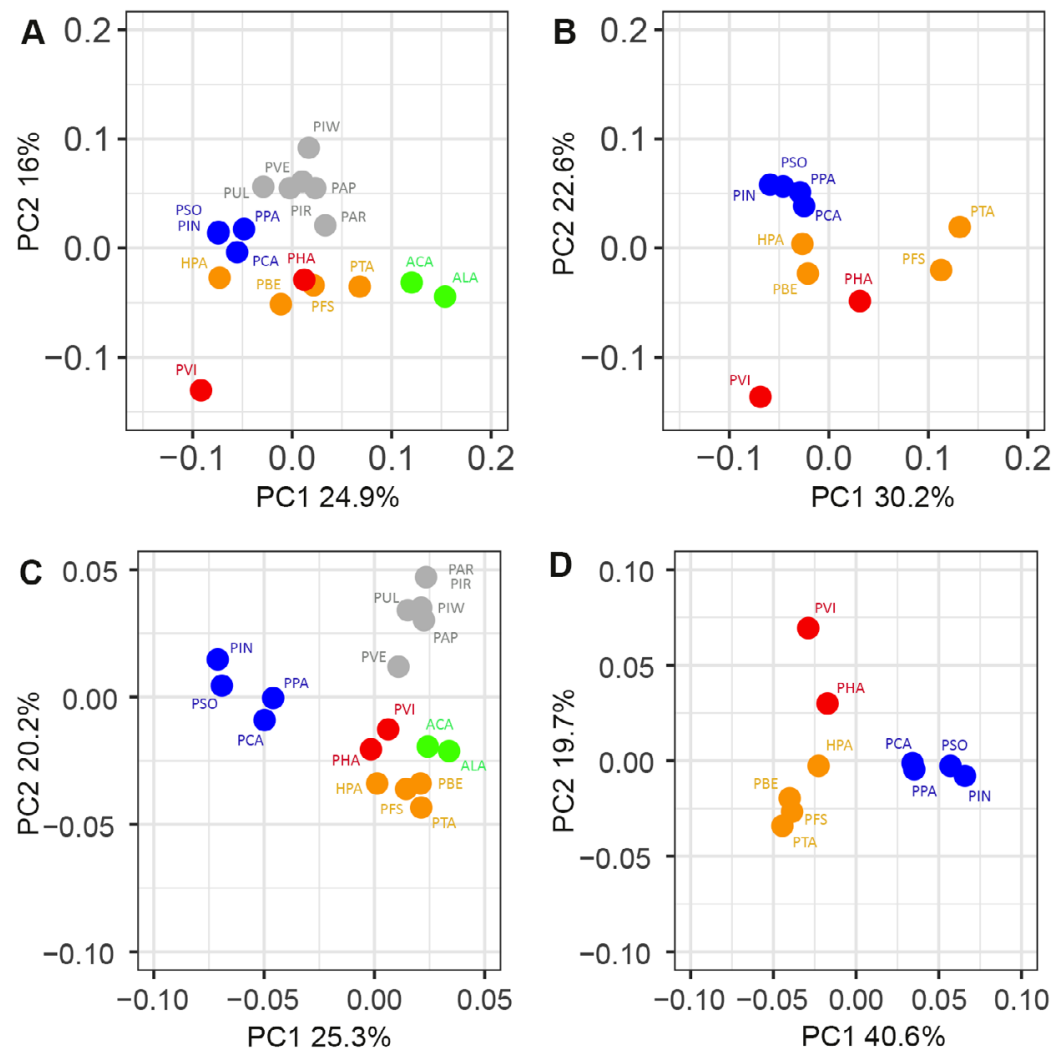

\footnotetext{
Hyaloperonospora/ Peronospora

Plasmopara
}

Phytophthora

Albugo

Pythium

\section{Figure 6. Principal component analysis (PCA) of variation in the}

\section{relative abundance of secreted CAZymes and Pfam domains.}

The variation in secreted CAZyme (AB) and Pfam (CD) domains along PC1 and PC2 is depicted in the figure. The PCAs include all of the 18 species (AC) or the Peronospora, Plasmopara and Phytophthora species only (BD). The PERMANOVA test shows that the grouping based on the CAZyme and Pfam domains is significant $(P<0.001)$. Species are grouped by color based on the classes that were defined in the phylogenetic tree (Fig. 5). Phytophthora (blue), Peronospora (yellow), Plasmopara (red), Albugo (green) and Pythium (grey). Abbr. PFS; Peronospora (P.) effusa, PBE; P. belbahrii, PTA; P. tabacina, HPA, Hyaloperonospora arabidopsidis, PHA; Plasmopara (PI.) halstedii, PVI; PI. vitiocola, PIN; Phytophthora (Ph.) infestans, PSO; Ph. sojae, PCA; Ph. capsici, PPA; Ph. parasitica, ACA; Albugo (A) candida, ALA; A. laibachii, PUL; Pythium (Py.) ultimum, PAR; Py. arrhenomanes, PAP; Py. Aphanidermatum, PIR; Py. irregulare, PIW; Py. lawyamai, PVE; Phytopythium vexans 
The CAZymes-based PCA supports the separate clusters of Albugo, Phytophthora and Pythium species as found in the core ortholog tree (Fig. 5). Remarkably, neither the Hyaloperonospora/Peronospora nor the Plasmopara species form a clear cluster, although the clustering is significant (PERMANOVA $p<0.001$ ). The variation along PC1 (Hyaloperonospora/Peronospora) and PC2 (Plasmopara) indicates that the secreted CAZyme domains vary largely between the species in these groups, despite their close phylogenetic relationship and same lifestyle.

2 The secreted CAZymes of the two Plasmopara species appear more similar to those of the Hyaloperonospora/Peronospora species than to the Phytophthora species, which is different from the results of the core ortholog protein comparison as shown in the phylogenetic tree (Fig. 5). To exclude the effect of the more distantly-related species on the separation between the downy mildew and Phytophthora species, the PCA was performed on the set without the Pythium and Albugo species (Fig. 6b). The pattern, as observed in the total set, is maintained when the more distantly related species are excluded from the analysis.

To look further into the properties of the secreted CAZymes we highlighted literature-curated domains of phytopathogenic oomycetes that are known to modify the main plant cell wall components; lignin, clulose and hemicellulose [69] (Fig. S4). We found that the secretomes differ more in terms of the absolute number of plant cell wall-degrading enzymes than in the relative occurrence of the different corresponding CAZyme catalytic activities per species. Secretomes of obligate biotrophic and hemibiotrophic/necrotrophic oomycetes have secreted proteins with similar functions (like breakdown of cellulose, pectin, hemicellulose etc.) but the numbers and diversity of those proteins in obligate biotrophic species are reduced.

The Pfam-based PCA shows a clear separation between lifestyles (Fig. 6cd). The Phytophthora species cluster together and separate from all other species along PC1 (25,3\%). The Pythium species form a cluster that separates clearly from the other species along PC2 $(20,2 \%)$. All biotrophic species, including both groups of downy mildews 
and the Albugo species, cluster together. Within the obligate biotrophic cluster the phylogenetic groups (Hyaloperonospora/Peronospora, Plasmopara, Albugo) as found in the core ortholog tree are still present but the differences are minor. To exclude the effect of the more distantly related species on the separation between the obligate biotrophs, the PCA was also performed without Pythium and Albugo species (Fig. 7b). The pattern observed in Fig. $6 \mathrm{C}$ is maintained when the more distantly related species are excluded from the analysis (Fig. 6d).

The repertoires of Pfam domains in the different groups of obligate biotrophs (Hyaloperonospora/Peronospora, Plasmopara and Albugo) are more similar than would be expected based on their taxonomic relationship. This could be the result of convergent evolution towards the obligate biotrophic lifestyle. Plasmopara and Hyaloperonospora/ Peronospora CAZyme repetoires are similar as well, but the Albugo species have a different CAZyme profile.

We conclude that a different composition and abundance in secreted Pfam domains is clearly associated with obligate biotrophy, suggesting it is the result of convergent evolution towards an obligate lifestyle.

To look further into the properties of the secreted CAZymes we highlighted literature-curated domains of phytopathogenic oomycetes that are known to modify the main plant cell wall components; lignin, cellulose and hemicellulose [174]. We found that the secretomes differ more in terms of the absolute number of plant cell wall-degrading enzymes than in the relative occurrence of the different corresponding CAZyme catalytic activities per species. Secretomes of obligate biotrophic and hemibiotrophic/necrotrophic oomycetes have secreted proteins with similar functions (like breakdown of cellulose, pectin, hemicellulose etc.) but the numbers and diversity of those proteins in obligate biotrophic species are reduced. 


\section{Seven Pfam domains contribute largely to the difference between obligate biotrophs and others.}

The Pfam domains that contribute to the variance in PC1 and PC2 were (Fig. 6 cd) identified using a biplot. In a biplot, the variables are presented as vectors, with their length reflecting their contribution. Many of the domains contribute to the differences between the biological groups, but seven of them stand out (Fig. 7. and Table 4).

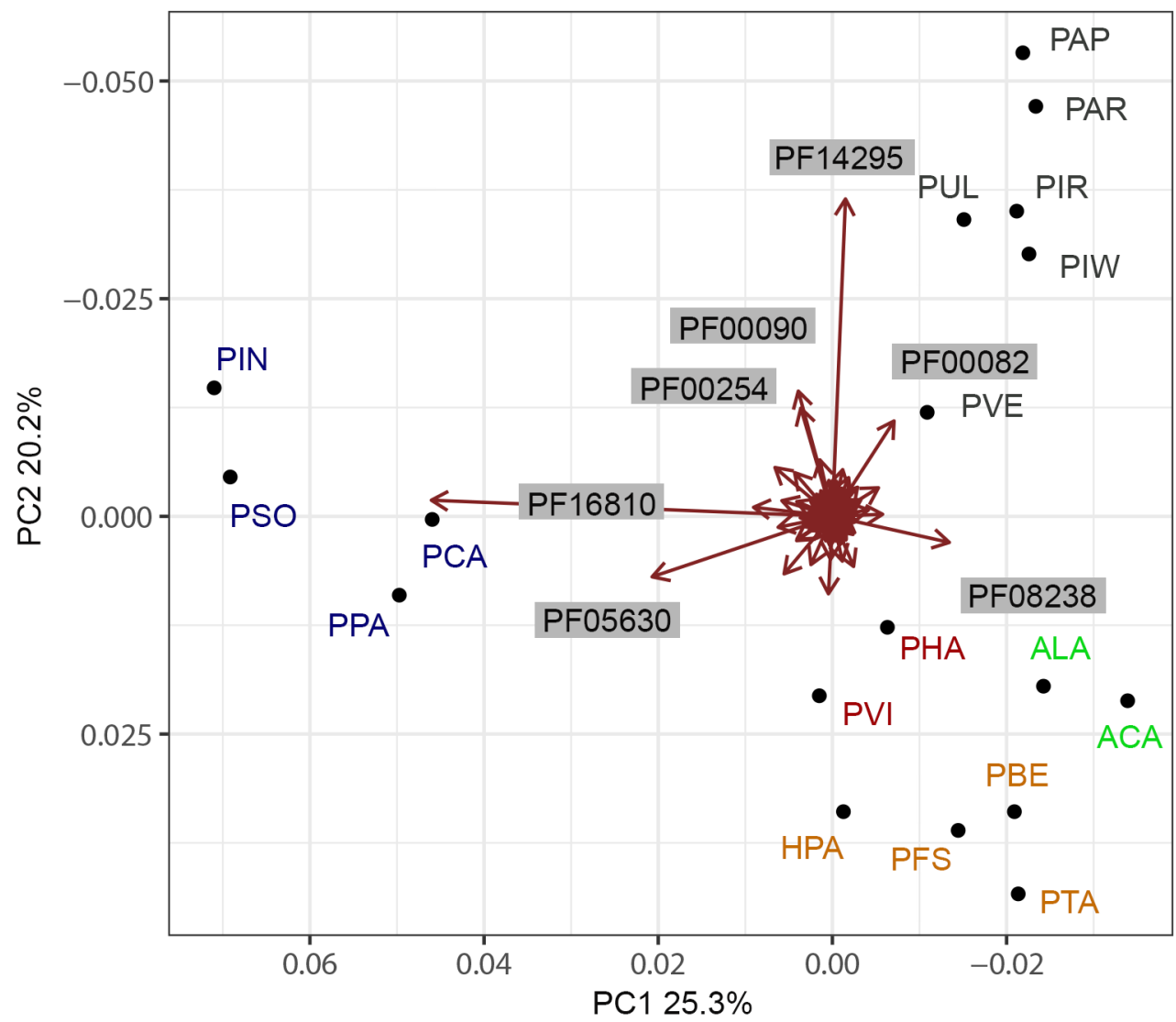

Figure 7. Pfam domains that strongly contribute to the variation in the relative abundance between species.

Although many domains contribute to the variation, PF16810, PF05630, PF08238, PF14295, PF00090, PF00254 and PF00082 are the domains that contribute most, as evidenced by the length of their vectors in the biplot. 
Two Pfam domains that have a higher relative abundance in Phytophthora contribute strongly to the separation between Phytophthora and the other species. The first, PF16810, represents a RxLR protein family with a conserved core $\alpha$-helical fold (WY-fold). Some of the proteins that this domain was based on have a known avirulence activity [77], i.e. they are recognized by plant resistance proteins. On average, 82 PF16810 domains were identified in Phytophthora species compared to 1.3 in Peronospora, 1.0 in Plasmopara and none in Albugo species. Using HMMer searches, many more WY-fold proteins can be identified in Plasmopara and Hyaloperonospora/Peronospora downy mildew species. However, these proteins do not match to the PF16810 Pfam domain that is based on a larger protein sequence as the HMM. 


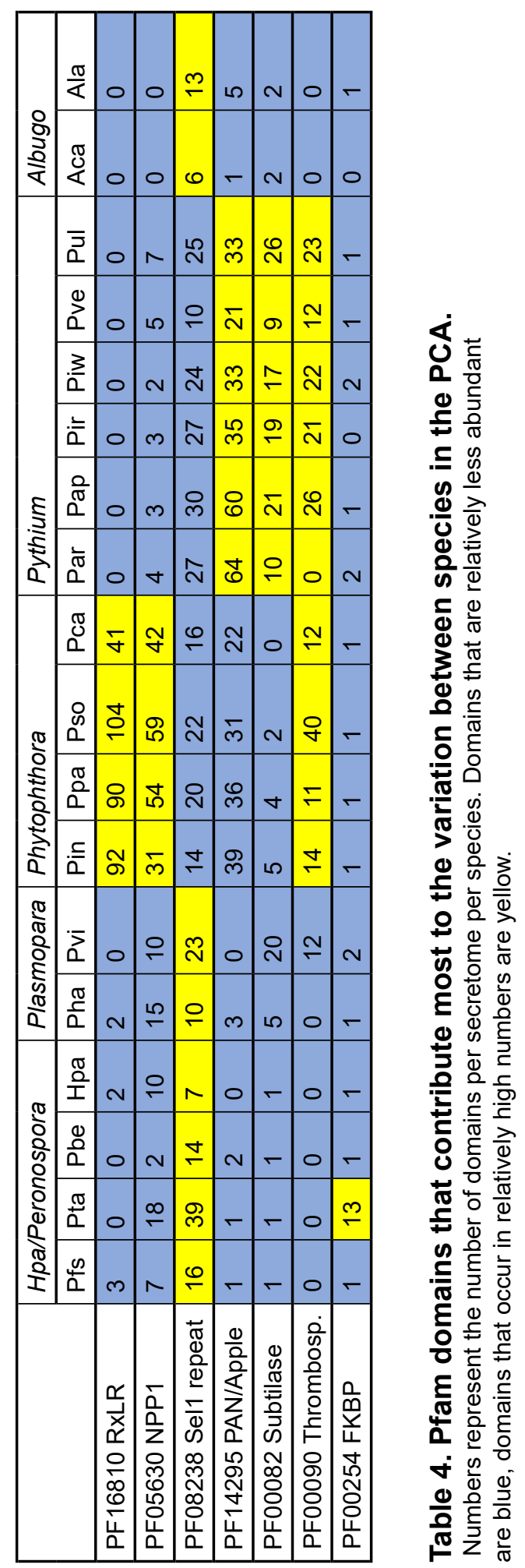


The second, PF05630, is a necrosis-inducing protein domain (NPP1) that is based on a protein of $P h$. parasitica [175]. This domain is conserved in proteins belonging to the family of Nep1-like proteins (NLPs) that occur in bacteria, fungi and oomycetes [176]. Infiltration of cytotoxic NLPs in eudicot plant species results in cytolysis and cell death, visible as necrosis [177]. Phytophthora species are known to have high numbers of recently expanded NLP genes in their genomes, encoding both cytotoxic and non-cytotoxic NLPs [176]. $H$. arabidopsidis and other obligate biotrophs tend to have lower numbers and only encode noncytotoxic NLPs [52, 176].

Domain PF08238 contributes to the distance between the Phytophthora and obligate biotrophic species and is relatively more abundant in the biotrophs (PC1). PF08238 is a Sel1 repeat domain that is found in bacterial as well as eukaryotic species. Proteins with Sel1 repeats are suggested to be involved in protein or carbohydrate recognition and ER-associated protein degradation in eukaryotes [178]. No function of proteins with a PF08238 domain is known for oomycete or fungal pathogens.

The distance between Pythium and the obligate biotrophic species along PC2 is largely caused by differences in four domains that are commonly reported in oomycete secretomes [173]. The first, PF14295, a PAN/Apple domain, is known to be associated with carbohydrate-binding module (CBM)-containing proteins that recognize and bind saccharide ligands in Ph. parasitica. Loss of these genes, as in the biotrophs, may facilitate the evasion of host recognition as some CBM proteins are known to induce plant defense [179]. Second, PF00082, is a subtilase domain, which is found in a family of serine proteases. Secreted serine proteases are ubiquitous in secretomes of plant pathogens [180]. Secreted proteases from fungal species have been shown to enhance infection success by degrading plant derived antimicrobial proteins [181]. A third is PF00090, a Thrombospondin type 1 domain that is present in large numbers in the secretome of Phytophthora and Pythium species but is absent from the secretomes of Hyaloperonospora/Peronospora species and Plasmopara halstedii. The function of proteins with this domain in oomycetes or plants 
is unknown. Finally, PF00254 contributes to the separation along PC1, which seems mainly caused by 13 occurences of the domain in the secretome of $P$. tabacina versus 2 or less in the secretomes of the other oomycete species.

\section{Over and under-representation of Pfam domains in obligate biotrophic species}

Statistical analysis of enrichment of Pfam domains, to identify underand over-represented domains in each group (Hyaloperonospora/ Peronospora, Plasmopara, Albugo) compared to Phytophthora, confirmed the pattern that was shown in the biplot. In total, 60 Pfam domains were found to be differentially abundant in obligate biotrophic species clusters compared to Phytophthora (Table 5). All of the seven Pfam domains that contributed most to the separation between phylogenetic groups in the PCA (Fig. 7 and Table 4) were also found to be differentially abundant in at least one obligate biotrophic cluster compared to Phytophthora in the enrichment analysis.

Previous studies identified Pfam domains that are associated with virulence in other phytopathogenic oomycete species like Pythium, Plasmopara, Peronospora and Phytophthora [182]. The occurrence of these known virulence-associated domains in the Pfs proteome is summarized in Fig. S5. We found that obligate biotrophic species have a lower total, as well as relative, number of secreted proteins with virulenceassociated domains compared to the other oomycete species. 
Table 5. Over and under-representation of Pfam domains in the secretomes of Hyaloperonospora/Peronospora (HP), Plasmopara (PI) and Albugo (Al) compared to Phytophthora species.

\begin{tabular}{|c|c|c|c|c|c|}
\hline Pfam & Name & Interpro\# & HP & PI & Al \\
\hline PF16810 & $\begin{array}{l}\text { RxLR protein, } \\
\text { Avirulence activity }\end{array}$ & IPR031825 & $8,30 e^{-24}$ & $2,70 e^{-16}$ & $3,90 e^{-07}$ \\
\hline PF14295 & PAN domain & IPR003609 & $1,80 e^{-07}$ & $1,80 e^{-04}$ & 16,913 \\
\hline PF00090 & $\begin{array}{l}\text { Thrombospondin type } \\
1\end{array}$ & IPR000884 & $4,50 e^{-05}$ & 77,4987 & 1,98225 \\
\hline PF05630 & $\begin{array}{l}\text { Necrosis inducing } \\
\text { protein (NPP1) }\end{array}$ & IPR008701 & $1,60 e^{-01}$ & 1,36788 & $2,13 e^{-03}$ \\
\hline PF08238 & Sel1 repeat & IPR006597 & $1,70 e^{-07}$ & 4,61559 & 1,07208 \\
\hline PF00254 & $\begin{array}{l}\text { FKBP-type cis-trans } \\
\text { isomerase }\end{array}$ & IPR001179 & $1,80 e^{-04}$ & & \\
\hline PF00050 & $\begin{array}{l}\text { Kazal-type serine } \\
\text { protease inhibitor }\end{array}$ & IPR002350 & $2,05 e^{-03}$ & & \\
\hline PF07974 & EGF-like domain & IPR013111 & $1,23 e^{-02}$ & & \\
\hline PF13456 & $\begin{array}{l}\text { Reverse transcriptase- } \\
\text { like }\end{array}$ & IPR002156 & $2,60 e^{-10}$ & & \\
\hline PF00300 & $\begin{array}{l}\text { Histidine phosphatase } \\
\text { superfamily }\end{array}$ & IPR013078 & $7,74 e^{-03}$ & & \\
\hline PF00665 & Integrase core domain & IPR001584 & $1,07 e^{-02}$ & & \\
\hline PF00571 & CBS domain & IPR000644 & $1,66 e^{-02}$ & & \\
\hline PF00089 & Trypsin & IPR001254 & & $1,10 e^{-12}$ & \\
\hline PF01833 & IPT/TIG domain & IPR002909 & & $8,00 e^{-12}$ & \\
\hline PF00082 & Subtilase family & IPR000209 & & $3,10 e^{-10}$ & \\
\hline PF01341 & $\begin{array}{l}\text { Glycosyl hydrolases } \\
\text { family } 6\end{array}$ & IPR016288 & & $3,30 e^{-05}$ & \\
\hline PF00182 & Chitinase class I & IPR000726 & & $2,60 e^{-04}$ & \\
\hline PF01670 & $\begin{array}{l}\text { Glycosyl hydrolase } \\
\text { family } 12\end{array}$ & IPR002594 & & $1,09 e^{-03}$ & \\
\hline PF03184 & $\begin{array}{l}\text { DDE superfamily } \\
\text { endonuclease }\end{array}$ & IPR004875 & & & $2,40 e^{-06}$ \\
\hline PF09818 & $\begin{array}{l}\text { Predicted ATPase of } \\
\text { the ABC class }\end{array}$ & IPR019195 & & & $4,60 e^{-06}$ \\
\hline PF00169 & $\mathrm{PH}$ domain & IPR001849 & & & $8,10 e^{-06}$ \\
\hline PF01764 & Lipase (class 3) & IPR002921 & & & $1,30 e^{-04}$ \\
\hline
\end{tabular}


Table 5. continued

\begin{tabular}{|c|c|c|c|c|c|}
\hline Pfam & Name & Interpro\# & HP & PI & Al \\
\hline PF00026 & $\begin{array}{l}\text { Eukaryotic aspartyl } \\
\text { protease }\end{array}$ & IPR033121 & & & $2,30 e^{-04}$ \\
\hline PF13405 & EF-hand domain & IPR002048 & & & $3,70 e^{-04}$ \\
\hline PF15924 & $\begin{array}{l}\text { ALG11 } \\
\text { mannosyltransferase }\end{array}$ & IPR031814 & & & $3,70 e^{-04}$ \\
\hline PF01546 & $\begin{array}{l}\text { Peptidase family M20/ } \\
\text { M25/M40 }\end{array}$ & IPR002933 & & & $3,70 \mathrm{e}^{-04}$ \\
\hline PF07687 & $\begin{array}{l}\text { Peptidase dimerisation } \\
\text { domain }\end{array}$ & IPR011650 & & & $3,70 e^{-04}$ \\
\hline PF03870 & $\begin{array}{l}\text { RNA polymerase } \\
\text { Rpb8 }\end{array}$ & IPR005570 & & & $3,70 e^{-04}$ \\
\hline PF13041 & PPR repeat family & IPR002885 & & & $3,70 e^{-04}$ \\
\hline PF00443 & $\begin{array}{l}\text { Ubiquitin carboxyl- } \\
\text { terminal hydrolase }\end{array}$ & IPR001394 & & & $1,38 e^{-03}$ \\
\hline PF10152 & $\begin{array}{l}\text { Subunit CCDC53 of } \\
\text { WASH complex }\end{array}$ & IPR019309 & & & $1,63 e^{-03}$ \\
\hline PF00041 & $\begin{array}{l}\text { Fibronectin type III } \\
\text { domain }\end{array}$ & IPR003961 & & & $2,29 \mathrm{e}^{-03}$ \\
\hline PF07727 & Reverse transcriptase & IPR013103 & & & $6,09 e^{-03}$ \\
\hline PF04130 & Spc97 / Spc98 family & IPR007259 & & & $2,15 \mathrm{e}^{-02}$ \\
\hline PF01753 & MYND finger & IPR002893 & & & $2,15 e^{-02}$ \\
\hline PF03577 & Peptidase family C69 & IPR005322 & & & $2,15 e^{-02}$ \\
\hline PF03388 & $\begin{array}{l}\text { Legume-like lectin } \\
\text { family }\end{array}$ & IPR005052 & & & $3,02 e^{-02}$ \\
\hline PF03133 & $\begin{array}{l}\text { Tubulin-tyrosine ligase } \\
\text { family }\end{array}$ & IPR004344 & & & $3,02 e^{-02}$ \\
\hline PF13181 & $\begin{array}{l}\text { Tetratricopeptide } \\
\text { repeat }\end{array}$ & IPR019734 & & & $3,02 e^{-02}$ \\
\hline PF01156 & Nucleoside hydrolase & IPR001910 & & & $3,02 e^{-02}$ \\
\hline PF06367 & $\begin{array}{l}\text { Diaphanous FH3 } \\
\text { Domain }\end{array}$ & IPR010472 & & & $3,02 e^{-02}$ \\
\hline PF04910 & $\begin{array}{l}\text { Transcriptional } \\
\text { repressor TCF25 }\end{array}$ & IPR006994 & & & $3,02 \mathrm{e}^{-02}$ \\
\hline
\end{tabular}


Table 5. continued

\begin{tabular}{|c|c|c|c|c|c|}
\hline Pfam & Name & Interpro" & HP & PI & Al \\
\hline \multirow[t]{2}{*}{ PF00044 } & $\begin{array}{l}\text { Glyceraldehyde 3-ph. } \\
\text { dehydrogenase }\end{array}$ & IPR020828 & & & $3,02 e^{-02}$ \\
\hline & Glyceraldehyde 3-ph. & & & & $3,02 e^{-02}$ \\
\hline PF02800 & dehydrogenase & IPR020829 & & & \\
\hline \multirow[t]{2}{*}{ PF01428 } & AN1-like Zinc finger & IPR000058 & & & $3,02 e^{-02}$ \\
\hline & Electron transfer FAD- & & & & $3,02 e^{-02}$ \\
\hline \multirow[t]{2}{*}{ PF00766 } & binding domain & IPR014731 & & & \\
\hline & Electron transfer & & & & $3,02 e^{-02}$ \\
\hline \multirow[t]{2}{*}{ PF01012 } & flavoprotein domain & IPR014730 & & & \\
\hline & UPF0160 & & & & $3,02 e^{-02}$ \\
\hline \multirow[t]{2}{*}{ PF03690 } & (uncharacterized) & IPR003226 & & & \\
\hline & Helicase C-terminal & & & & $3,02 e^{-02}$ \\
\hline \multirow[t]{2}{*}{ PF13307 } & domain & IPR006555 & & & \\
\hline & Microtubule-binding & & & & $3,02 e^{-02}$ \\
\hline PF08683 & calmodulin-reg & IPR014797 & & & \\
\hline PF01846 & FF domain & IPR002713 & & & $3,02 e^{-02}$ \\
\hline PF13418 & Galactose oxidase & & & & $3,02 e^{-02}$ \\
\hline \multirow[t]{2}{*}{ PF03776 } & MinE & IPR005527 & & & $3,02 e^{-02}$ \\
\hline & Iguana/Dzip1-like & & & & $3,02 \mathrm{e}^{-02}$ \\
\hline \multirow[t]{2}{*}{ PF13815 } & DAZ-interacting & IPR032714 & & & \\
\hline & Type III restriction & & & & $3,02 \mathrm{e}^{-02}$ \\
\hline PF04851 & enzyme & IPR006935 & & & \\
\hline \multirow[t]{2}{*}{ PF13831 } & PHD-finger & & & & $3,02 e^{-02}$ \\
\hline & Arp2/3 complex, p34- & & & & $3,02 e^{-02}$ \\
\hline PF04045 & Arc & IPR007188 & & & \\
\hline \multirow[t]{2}{*}{ PF08144 } & CPL (NUC119) domain & IPR012959 & & & $3,02 e^{-02}$ \\
\hline & POLO box duplicated & & & & $3,02 \mathrm{e}^{-02}$ \\
\hline \multirow[t]{3}{*}{ PF00659 } & region & IPR000959 & & & \\
\hline & SMP-30/ & & & & $4,76 \mathrm{E}^{-02}$ \\
\hline & Gluconolaconase/ & & & & \\
\hline PF08450 & LRE-like & IPR013658 & & & \\
\hline
\end{tabular}

Over (green) and under (blue)-representation was tested relative to the expected distribution of each Pfam domain. The abundance of each domain was compared between the species clusters using a Chi-square test with Bonferroni correction. Bonferroni

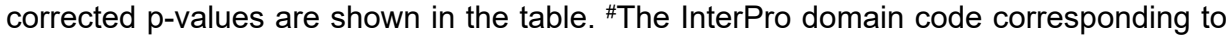
each Pfam domain is provided. 


\section{Host-translocated effectors}

The RxLR effector models in the Pfam database (PF16810 and PF16829) mentioned above cover only a small fraction of the predicted RxLR effectors in secretomes of phytopathogenic oomycetes. We predicted the total number of host-translocated effectors for each secretome using a Perl regex script and HMM searches (see methods), including RxLR effectors without WY domains and CRN effectors (Fig. 8). RxLR effector proteins were more abundant in Phytophthora compared to the obligate biotrophic species. On average $399 \mathrm{RxLR}$ effector proteins were found in Phytophthora whereas Plasmopara and Hyaloperonospora/Peronospora had 79 and 90 . The same pattern is evident for CRN effectors. The average number of CRN proteins in Hyaloperonospora/Peronospora is 11, while Plasmopara has 12 and Phytopthora 56. We conclude that downy mildew species (Hyaloperonospora/Peronospora and Plasmopara) have fewer host-translocated effectors compared to Phytophthora species. 
A

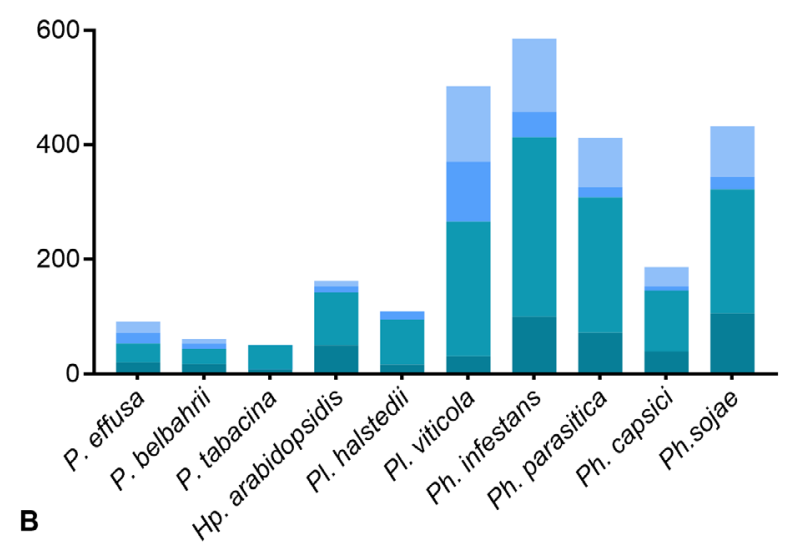
RxLR-(like) $+W Y+E E R(-$ like $)$
D RxLR(-like) $+W Y$
- RxLR + EER (-like)
- RxLR

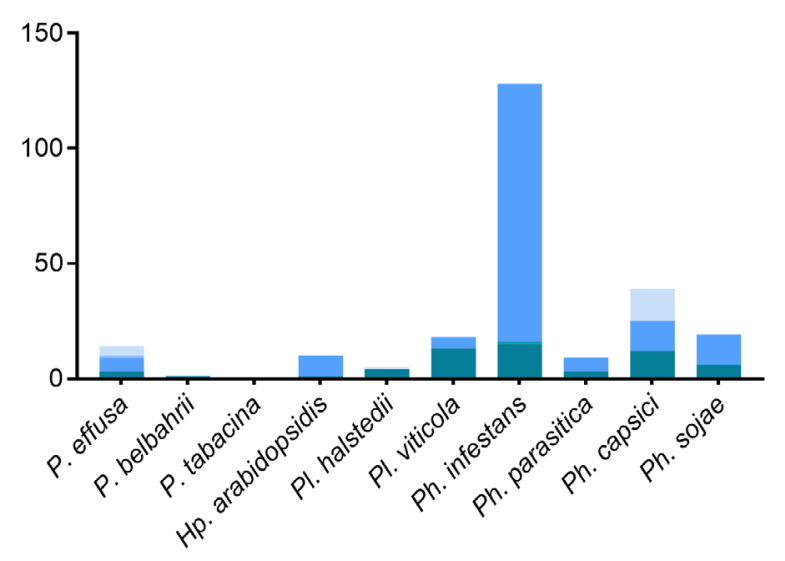
- LFLAK (-like) $+\mathrm{HVL}+\mathrm{HMM}$
LFLAK $(-$ like $)+$ HVL
LFLAK (-like) +HMM
- LFLAK
- HMM

Figure 8. Predicted (a) RxLR and (b) CRN effectors in the secretome of Hyaloperonospora/Peronospora, Plasmopara and Phytophthora species.

The predicted effectors are classified into four (RxLR) or five (CRN) categories, based on the additional domains they possess. Please note that the number of Pfs effectors is slightly different from the numbers reported before (Fig. S3). For this comparison we used HMM models that were previously published rather than the models trained for Pfs (Fig. S3). 


\section{Discussion \\ Taxonomic filtering}

The ability to sequence full genomes at high pace and relatively low cost has aided research in phytopathology dramatically. Over the past few years, the genomes of many phytopathogenic oomycetes have been sequenced and their genomes revealed an arsenal of protein coding genes with a putative virulence role. However, technical difficulties restricted the

2 sequencing and assembly of genomes of obligate biotrophic oomycetes that cannot be cultured axenically. Obligate biotrophic species can only grow on living host tissue so when collecting spores for DNA isolation DNA of other microbes and the host plant will inevitably contaminate the sample, which complicates the genome assembly. In this paper we use a metagenome filtering method resulting in the assembly of a relatively clean genome sequence of the obligate biotrophic downy mildew of spinach, Peronospora effusa.

To get a clean assembly, sequence that are derived from different species were filtered out and removed. Several methods were considered to identify and filter contigs or reads that were likely contaminants in our data. Initially we considered to filter contigs or reads based on their GC content, since this differs between genomes of oomycetes [183] and many other microbes [184]. In addition, the GC content is not constant over the genome, so filtering based on this could potentially remove valuable parts of the genome.

Alternatively, reads of non-oomycete origin could be identified by mapping them to databases with sequences of known taxonomy. For example, a database containing only oomycete or bacterial genomes. This is not ideal as the databases are incomplete and are likely to contain annotation errors. In addition, it could lead to the removal of novel parts of the downy mildew genome that are not present in other oomycetes, and which would hamper the study of valuable species-specific parts of your genome. 
The filtering we applied with the CAT tool does not classify a contig based on a single hit. Instead it determines the taxonomic origin of each ORF on an assembled contig or corrected PacBio read, providing a robust classification [122]. In our Pfs study, after filtering with the CATpipeline of the error-corrected $\mathrm{PacBio}$ reads, $50 \%$ remained, and were used in the assembly. Of the sequenced (unfiltered) Illumina reads, $56 \%$ could be aligned to the final assembly. This indicates that roughly half of our sequencing reads originated from other sources besides Pfs. Notably, while the classifications in the original CAT paper were only benchmarked on prokaryotic sequences [122], our study shows that the tool also performs well for classifying eukaryotic contigs. Thus, CAT may also be promising for classification of eukaryotes including oomycetes in metagenomic datasets, provided that long contigs, or corrected PacBio or Nanopore sequencing reads are available.

It should be noted that sequences of unknown taxonomy were maintained for the assembly, making it possible that these are still contaminants. When we compare the taxonomic distributions generated by Kaiju of the pre-assembly and final assembly, we see a dramatic reduction of sequences of bacterial origin (Fig. 3). The oomycete content according to Kaiju and the overall GC content of the final assembly is similar to that of genome assemblies of axenically-grown oomycetes. We can therefore conclude that the CAT filtering method, allowed the successful removal of sequences of non-oomycete origin.

\section{Hybrid assembly}

Most oomycete genomes sequenced to date were found to contain long repeat regions [185] that cannot be resolved using only a shortread technology such as Illumina. Long reads can potentially sequence over long repeats, and contribute to the contiguity of a genome assembly [186]. Therefore, our Illumina data was complemented with long read PacBio sequences in an attempt to close gaps between contigs. Although the inclusion of PacBio reads in our assembly improved the contiguity, the final result still consists of a large number of contigs, indicating that 
our PacBio reads were unable to span many repeat regions. Besides biological reasons for the large number of contigs, there could also be a technical reason. Prior to PacBio sequencing whole genome amplification (WGA) with random primers was performed as the initial sequencing attempt with non-amplified DNA barely yielded sequencing reads. WGA might create a bias, where some parts of the oomycete genome may be under-represented in the PacBio data.

\section{The genome of Pfs 1}

The assembled Pfs1 genome size is $32.4 \mathrm{Mb}$ divided over of 8,635 contigs. The genome is highly gene dense and contains in total 13,227 genes. Overall, the BUSCO analysis showed that this assembly contains most of the gene-space. Many of the 8,635 contigs were smaller than 1 $\mathrm{kb}$. However, the CAT filtering method performs best on relatively large contigs containing multiple ORFs. Therefore, small contigs could still contain sequences derived from other organisms. The removal of these small contigs results in only a small genome size reduction (1.9 Mb) and loss of gene models (597), but significantly reduces the number of contigs (by 5,027$)$. When we also account for genes that have a significant overlap (>20\%) with repeats in the genome (3983 gene models), or that were annotated as transposable elements (36 gene models that did not had an overlap with a repeat region) we come to 8,976 high-confidence gene models.

The genomes of $P f s$ race 13 and 14 have recently been published $[187,188]$, with a similar genome size $(32.1 \mathrm{Mb}$, and $30.8 \mathrm{Mb}$ respectively) and gene content ( 8000 gene models) compared to our Pfs1 genome assembly. Contrary to our assembly method, the input data for those genome assemblies were filtered by alignment to an oomycete and bacterial database to discard reads that do not belong to the oomycete genus. This filtering method could potentially lead to the incorporation of bacterial sequences that are not in the public databases. Besides, the positive filtering for oomycete scaffolds against NCBI nt database could have resulted in the loss of Pfs specific genome sequences. In addition, 
by filtering reads based on a database containing bacterial and fungal sequences, part of the Pfs genome yielded by horizontal gene transfer (HGT) may be discarded [189]. The CAT-tool overcomes this issue by determining the overall taxonomy of larger contigs based on multiple genes.

\section{Peronospora species have reduced genomes}

Recent sequencing of Peronospora species shows that they have remarkably small and compact genomes $(32.3-63.1 \mathrm{Mb})$ compared to Phytophthora (82- $240 \mathrm{Mb}$ ) species [5, 58, 60, 187]. The $k$-mer analysis predicts the Pfs1 genome to be $36.2 \mathrm{Mb}$ containing $8.8 \mathrm{Mb}$ of repeats (24\%). The predicted genome size of Pfs $R 13$ and $R 14$ based on $k$-mer analysis is $44.1-41.2 \mathrm{mb}$ (repeats; $24-22 \%$ ) [187]. The increased genome size of Phytophthora is attributed to an ancestral whole genome duplication in the lineage leading to Phytophthora and to an increase in the proportion of repetitive non-coding DNA [58, 190]. The duplication event has been proposed to have taken place after the speciation of $H$. arabidopsidis [191]. However new multigene phylogenies show that the Peronospora lineage has speciated after the divergence of Phytophthora clade 7 from clade 1 and 2. Notably, these three clades all contain species with duplicated genomes [19, 22, 27, 192]. This would suggest that an ancestral whole genome duplication before this speciation point would also apply to Peronospora, and would mean that duplication cannot account for the difference in genome size. The availability now of genomes of three Peronospora species for comparisons asks for a reevaluation of the timing of the duplication and subsequent speciation events.

Biologically, the question of how Peronospora species can be host-specific and obligate biotrophic while maintaining only a small and compact genome is interesting. It is argued that the trend in filamentous phytopathogens is towards large genomes with repetitive stretches to enhance genome plasticity [190]. Plasticity may enable host jumps and 
adaptations that favor the species for survival over species with small, less flexible genomes [190]. The reduced genomes of Peronospora species show an opposing trend that cannot be attributed to their obligate biotrophic lifestyle alone, as it is not evident in Plasmopara species (75 $\mathrm{Mb}-92 \mathrm{Mb}$ ) [22, 193]. Sequencing of multiple isolates of the same Peronospora species may shed light on genome plasticity at the species level.

\section{Secretome reflects biotrophic lifestyle Evolving biotrophy}

The biotrophic lifestyle has emerged on several independent occasions in the evolution of filamentous plant pathogens, in several branches of the tree of life. Convergent evolution is thought to be the main driving factor behind the development of biotrophy in such distantly related organisms [194]. However, it was shown that horizontal gene transfer can also occur between fungi and oomycetes, resulting in 21 fungal proteins in the secretome of $H$. arabidopsidis. Out of these 21 proteins, 13 were predicted to secreted, indicating that horizontal gene transfer may affect a species pathogenicity and interaction with the host $[195,196]$.

It was proposed that the critical step for adopting biotrophy in filamentous phytopathogens is the ability to create and maintain functional haustoria [63]To do so, a species needs to be able to avoid host recognition or suppress the host defense response. A proposed mechanism for avoidance of host recognition is the loss of proteins involved in cell wall degradation, as evidenced by the reduction of cell wall degrading enzymes in mutualistic species compared to biotrophs [197]. In this and other studies, we find a reduction of the number of cell wall degrading enzymes in obligate biotrophic species compared to hemibiotrophic Phytophthora species (Fig. S3) [59]. This is true for all three obligate biotrophic groups in this study (Hyaloperonospora/Peronospora, Plasmopara and Albugo) although the difference is less clear in Plasmopara. Possibly this reduction is the result of a similar selection pressure to reduce recognition by the host plant in the biotrophic species, 
where the hemi-biotrophic nature of the interaction between host and Phytophthora allows for slightly less caution in recognition avoidance.

The other mechanism of establishing a strong interaction is suppression or avoidance of the host defense response. Biotrophic infections are often accompanied by co-infection of species that are unable to infect the plant in the absence of the biotroph, indicating efficient defense suppression [15, 63]. We found enhanced numbers of secreted serine proteases (PF00082) (suppression) and reduced numbers of proteins with PAN/Apple domains that are known to be recognized by the plant immune system.

While the expansion of host translocated RxLR effectors is evident in both hemi-biotrophic and biotrophic species, their numbers are smaller in secretomes of obligate biotrophs. CRN effectors are especially reduced in secretomes of biotrophic species. As opposed to RxLR effectors, CRNs are an ancient class of effectors that are known to induce cell death. Obligate biotrophic species presumably lost them as they are not beneficial for their survival.

In this study we first showed that the CAT tool performs well for taxonomic filtering of eukaryotic contigs. We provided a clean reference genome of a race 1 isolate of the spinach infecting downy mildew, Pfs1. In a comparative approach, we found that the secretomes of the obligate biotrophic oomycetes are more similar to each other than to more closely related hemi-biotrophic species when comparing the presence and absence of functional domains, including the host translocated effectors. We conclude that adaptation to biotrophy is reflected in the secretome of oomycete species. 


\section{Acknowledgments}

We wish to thank the Topsector Horticulture and Starting Materials (TKI) for funding the project. BED was supported by the Netherlands Organisation for Scientific Research (NWO) Vidi grant 864.14.004. We thank Ronnie de Jonge (Utrecht University) for useful input for the orthology analysis, Bjorn Wouterse for helping out with the comparative and statistical analysis, and the Utrecht Sequencing Facility for providing

2 sequencing service and data. Utrecht Sequencing Facility is subsidized by the University Medical Center Utrecht, Hubrecht Institute, Utrecht University and The Netherlands X-omics Initiative (NWO).

\section{Supplemental Tables}

S1 Table. Species used for comparative secretomics. Table S1 is available online:

https://doi.org/10.17026/dans-zey-8aec

S3 Table. Repeat elements in the Pfs1 genome. Repeat elements identified in the Pfs1 genome, for each repeat type the total numbers and percentage are shown. In addition, also a detailed annotation for each repeat element is provided.

Table S3 is available online:

https://doi.org/10.17026/dans-zey-8aec 

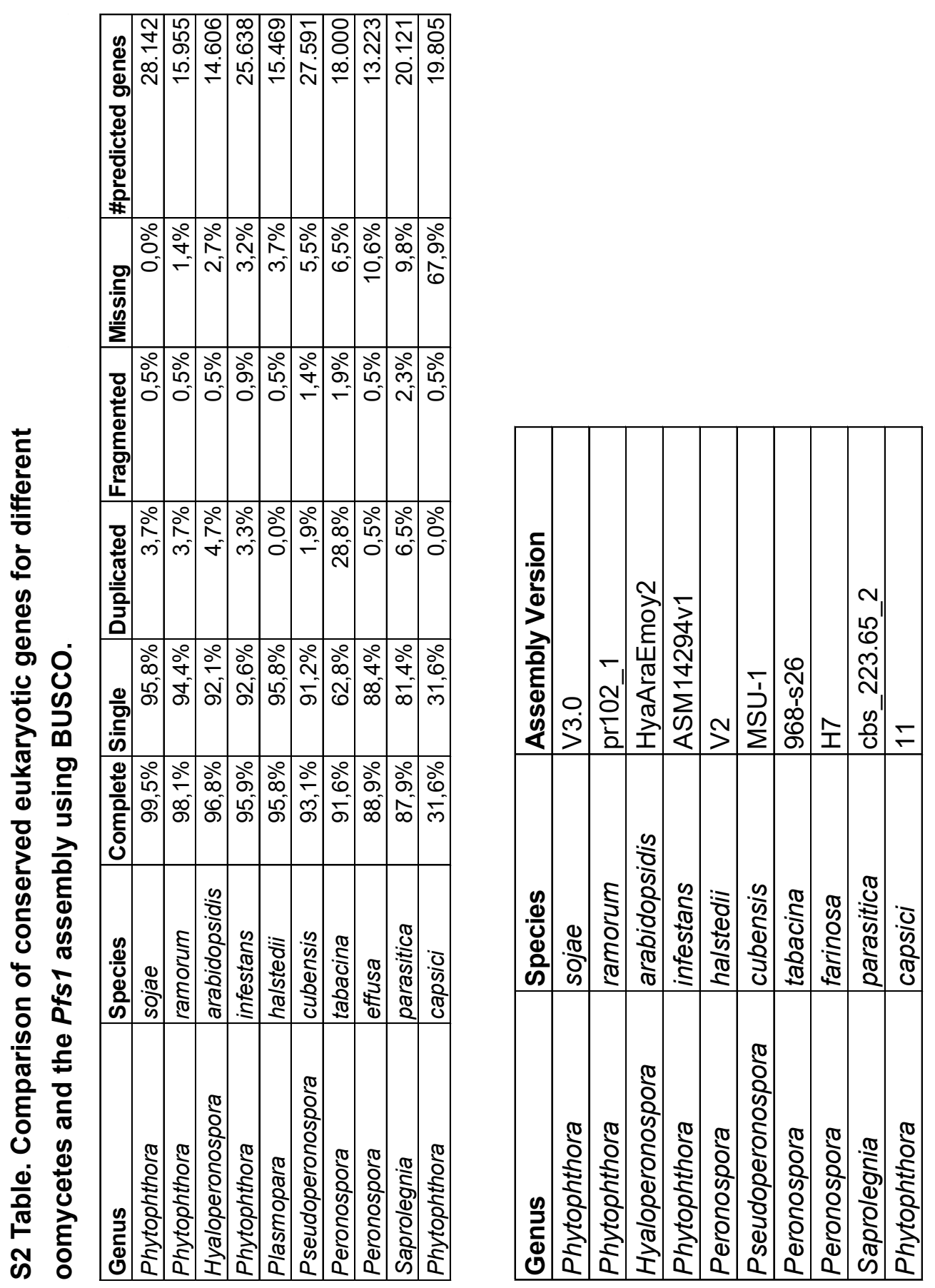


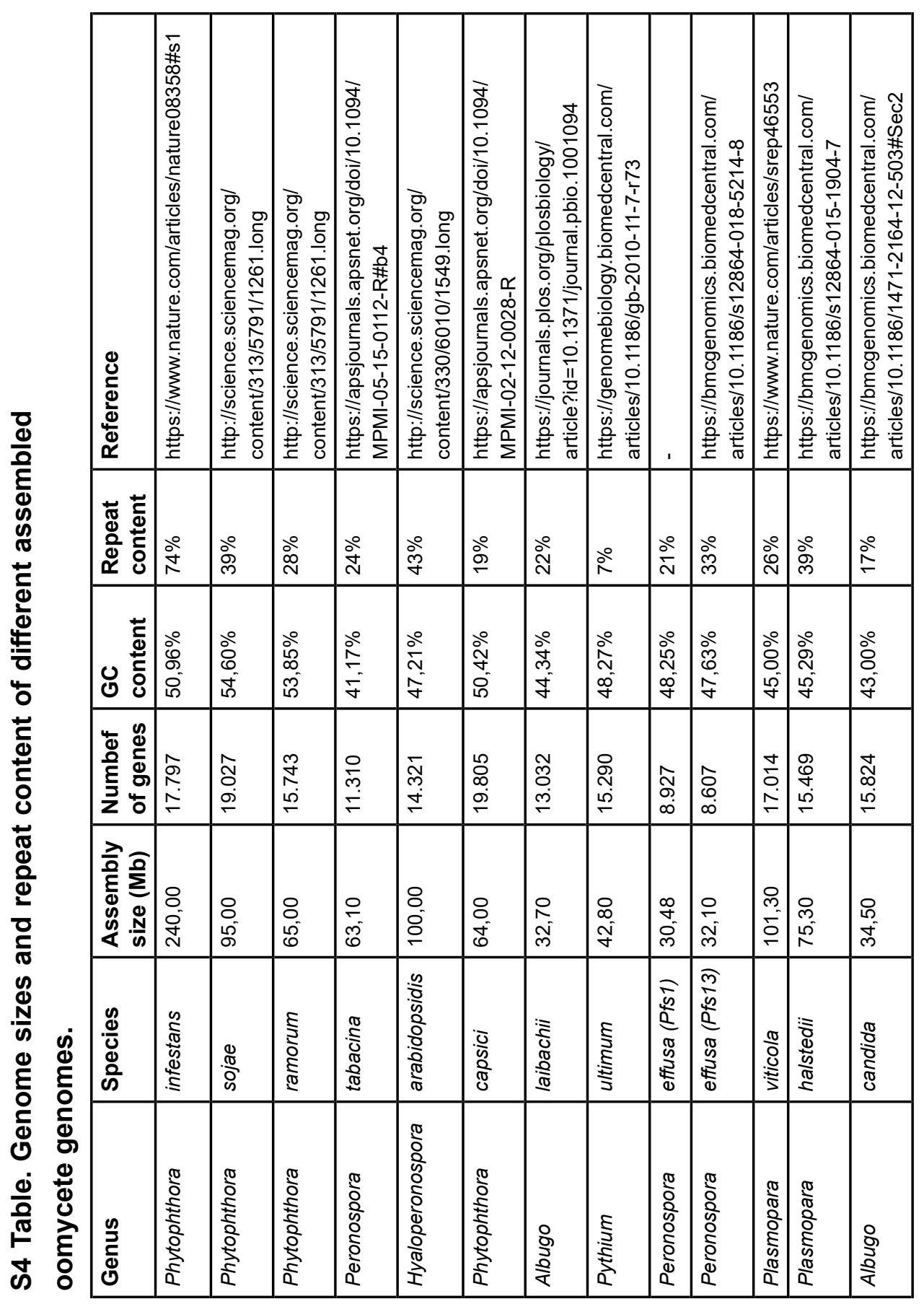


S5 Table. Putative annotations of the Pfs proteins as obtained with ANNIE. In addition, the presence of a N-terminal signal peptide for secretion, WY motif, TM motif and overlap with a repeat region are listed for each protein coding gene.

Table S5 is available online:

https://doi.org/10.17026/dans-zey-8aec

S6 Table. Overview of the host translocated effectors (RxLR and CRN) identified in the genome of Pfs1. Also, their respective functional domains and locations are listed per effector. Selected effectors that were used in the gene intergenic distance analysis are listed in the second tab.

Table S6 is available online:

https://doi.org/10.17026/dans-zey-8aec

S7 Table. Secreted CAZyme domains per species.

Table S7 is available online:

https://doi.org/10.17026/dans-zey-8aec

S8 Table. Secreted Pfam domains per species.

Table S8 is available online:

https://doi.org/10.17026/dans-zey-8aec 


\section{Supplemental Figures}
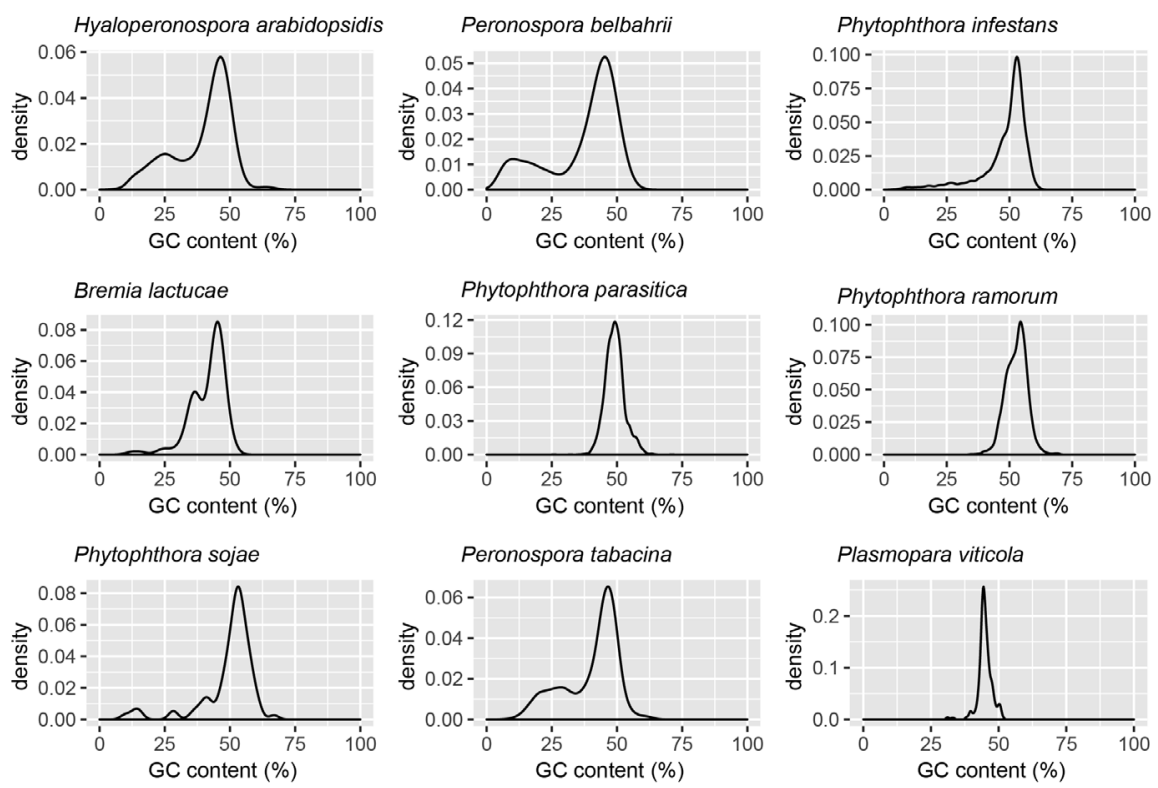

S1 Fig. GC plot of various oomycete assemblies on contigs larger than $1 \mathrm{~kb}$. 

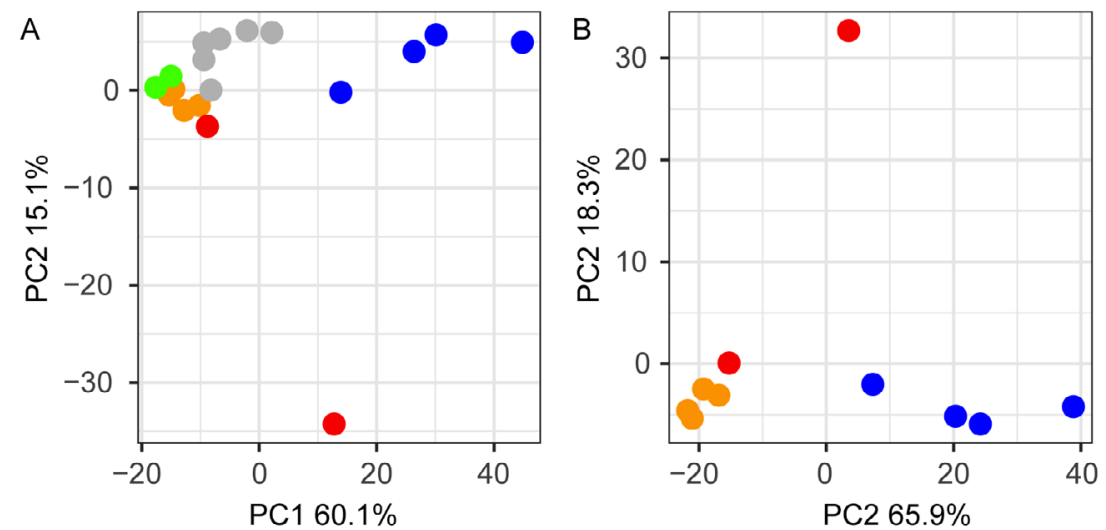

Hyaloperonosporal - Plasmopara
Peronospora

- Phytophthora Albugo Pythium

Figure S2. PCA on absolute numbers of secreted CAZyme domains.
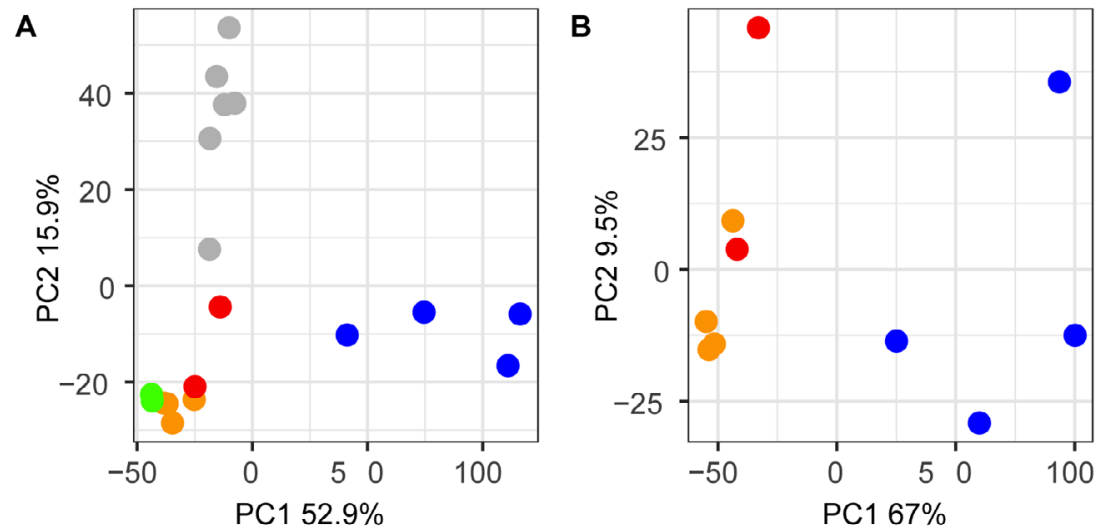

Hyaloperonospora/
Peronospora Plasmopara $\bullet$ Phytophthora Albugo $\bigcirc$ Pythium

Figure S3. PCA on absolute numbers of secreted Pfam domains. 
A
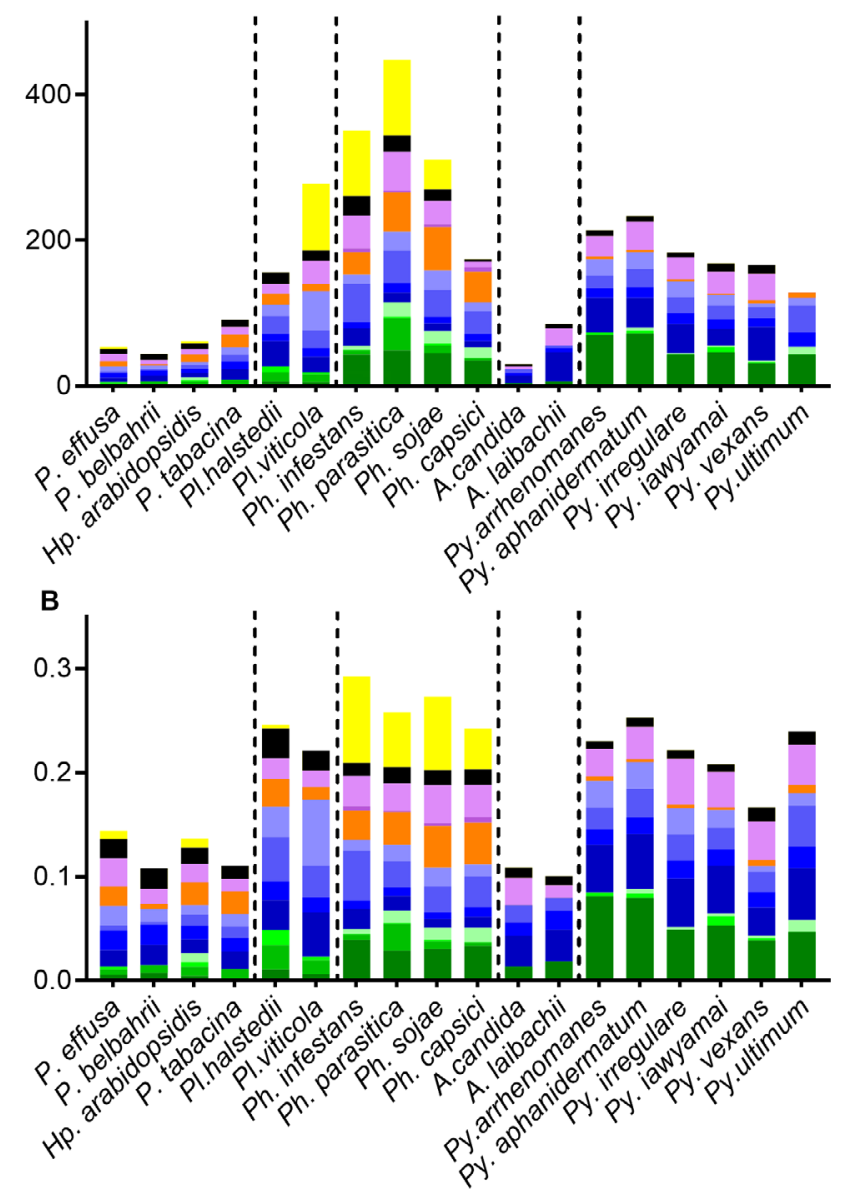

InterproRXLR

- cystein-rich secretory protein

elicitin

Pcf (Phytotoxin)

NPP1

- Trypsin

serine/cysteine protease inhibitor

a cysteine protease

- Protein degrading proteins

- Pectin lyase

- Ricin B lectin

- Jacalin

- CBEL

Figure S4. Secreted cell wall degrading proteins (CAZymes).

Numbers (a) of literature curated plant cell wall degrading enzymes per species. (b) The same data represented as fraction of the total number cell wall degrading protein domains per species. 
A

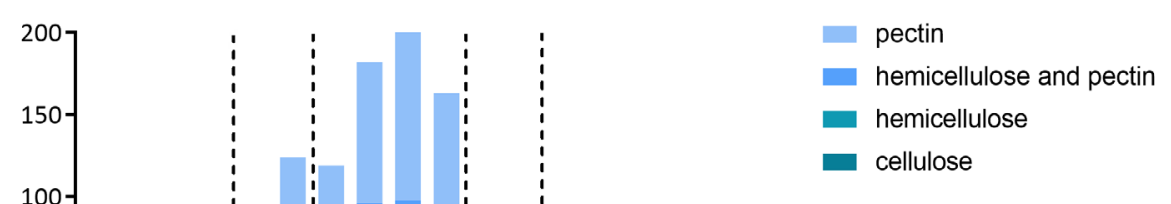

B

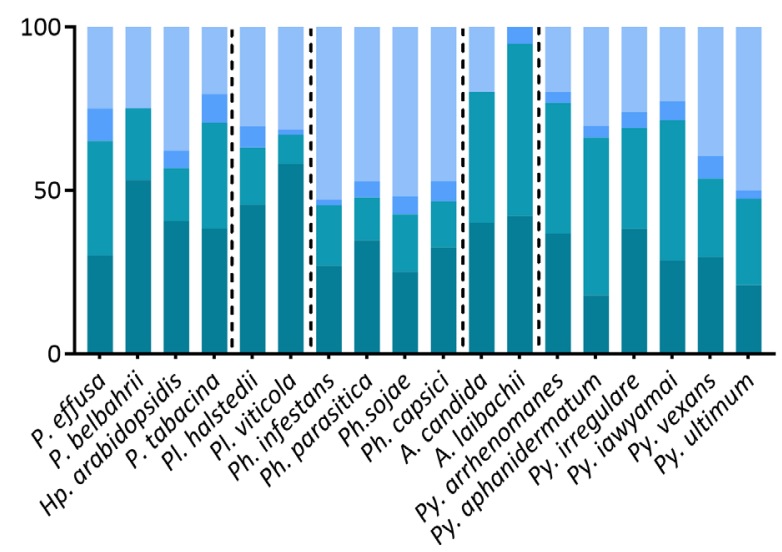

Figure S5. Secreted pathogenicity associated Pfam domains.

Occurrence of Pfam domains known to be involved in pathogenicity within the secretome of each species. Figure (a) shows the absolute number of Pfam domains, while (b) shows the number relative to the total number of Pfam domains per species. 

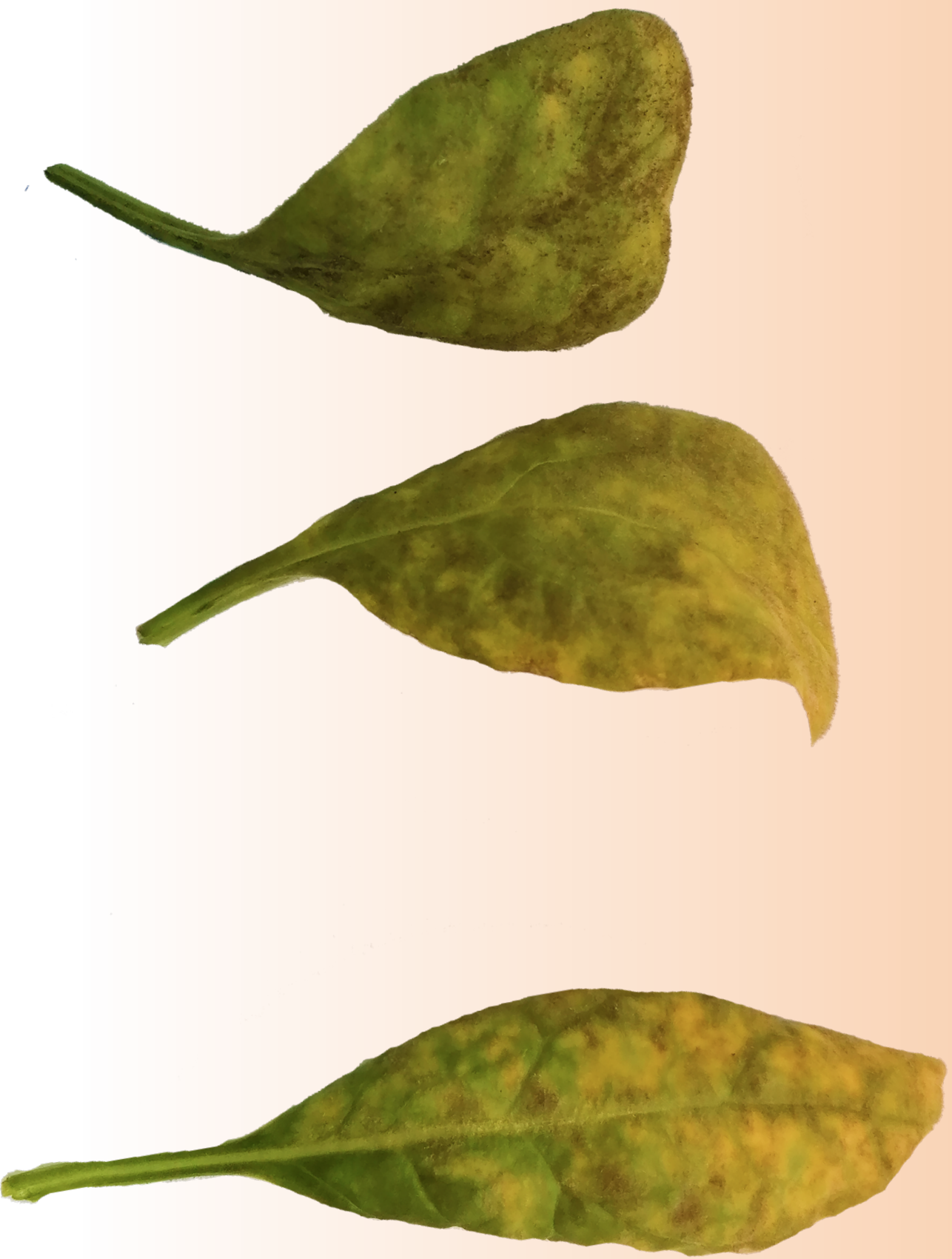


\section{3 \\ Identification of plant interactors of host- translocated effectors of Peronospora effusa}

Manon Neilen, Guido Van den Ackerveken

Plant-Microbe Interactions, Department of Biology, Utrecht University, Padualaan 8, 3584 CH Utrecht, the Netherlands 


\section{Abstract}

The obligate biotrophic oomycete Peronospora effusa (Pfs), which causes downy mildew on spinach, has an arsenal of host-translocated effectors that are presumed to manipulate plant cell processes during infection. Comparative analysis of its predicted proteome showed that 39 of them are unique to Pfs as they have no detectable orthologs in other species tested. Of the other candidate host-translocated effectors, 10 are downy mildews-specific, 23 occur in downy mildews and other Peronosporales species, and 40 are also found in more distantly-related oomycetes such as Pythium and Albugo species. Twenty-six candidate effectors were used as baits in a yeast two-hybrid screen of an Arabidopsis prey library. The screen revealed 40 interactions between 18 Pfs proteins and putative plant interactor proteins. An interaction network supplemented

3 with reported protein-protein interactions from the plant-pathogen literature showed that 9 interactors of Pfs effectors were also targeted by effectors of other species. These highly connected plant proteins like CSN5A, TCP14, and FBN1A likely play important regulatory roles in the plant cell during infection. Other Pfs effectors interact with less connected proteins. Some of these, like HBI1, two NAC transcription factors and two prenylated Rab acceptor (PRA1) proteins, have been linked to disease susceptibility in other species before but for most of them their biological function is unknown. This study identified the first plant proteins that interact with effectors of the spinach downy mildew and provides a first glimpse into the possible targets of host-translocated effectors of Pfs and their potential role in the infection process. 


\section{Introduction}

Phytopathogens secrete proteins and other molecules, named effectors, to establish and maintain successful infections. Effectors have evolved to manipulate plant processes to the benefit of the pathogen. An important activity of host-translocated effectors is to suppress plant immunity [198]. The plant immune system recognizes and responds to pathogenassociated molecular patterns (PAMPs) using receptors that trigger immune responses [80]. Pattern-triggered immunity (PTI) encompasses callose deposition, altered hormone signaling and gene transcription and the release of reactive oxygen species (ROS) and antimicrobial compounds, all to limit infection of plant tissues by pathogens [41, 42, 43].

Effector proteins of phytopathogens act in plant tissues either in the extracellular space (apoplastic effectors) or inside host cells (host-translocated effectors). Known activities of apoplastic effectors include inhibition of hydrolytic enzymes and proteases, induction of necrosis and protection of the pathogen cell wall [40], [199]. In oomycete phytopathogens two main types of host-translocated effector proteins are RxLR and Crinkler (CRN) effectors [55]. RXLR and CRN effectors are characterized by the presence of a signal peptide, a conserved RxLR or LFLAK (CRN) domain at the N-terminus and a variable $\mathrm{C}$-terminal effector domain which is responsible for the function of the effector in the host cell $[57,66]$. The RxLR and CRN motifs are often accompanied by EER and HVL motifs respectively [56, 200] and together they are likely required for the translocation into host cells $[56,65]$. Recently, it was shown that in an oomycete the RxLR motif likely guides effectors to a non-conventional secretion and translocation route that bypasses the oomycete Golgi system [69].

The C-terminal effector domain of CRN and RxLR effectors is responsible for the virulence function and usually lacks known protein domains or considerable conservation with other proteins [40]. However, a conserved tertiary structure, an a-helical fold, termed the WY-fold is found in Phytophthora and downy mildew species [60, 61, 77]. The WY- 
fold is hypothesized to provide molecular stability to the proteins, while maintaining flexibility to evolve and avoid recognition by the plant immune system [77]. For a number of host-translocated effectors it was shown that they interact with plant proteins to suppress immunity, cell death and RNA silencing [90, 103, 201, 202]. However, the virulence function of most host-translocated oomycete effectors is currently unknown.

Effector protein repertoires are highly variable between species. Effector genes are continuously lost (deleted), gained, and modified resulting in fast changing and dynamic gene repertoires and little conservation between effector repertoires of different species [75, 199]. RxLR effector proteins, for instance, have evolved and expanded within the Peronosporales resulting in hundreds of RxLR protein-encoding genes in the genomes of species like Phytophthora infestans (563),

3 Plasmopara viticola (>190) and Peronospora tabacina (120) [22, 58, 60, 203]. Rapid modification of effector proteins helps to avoid recognition by the plant immune system because pathogen success depends primarily on its ability to avoid recognition.

Although effector repertoires of different species have limited similarity, a number of host-translocated RxLR effectors are reported to be conserved among pathogenic oomycete species. Possibly, these effectors are especially important for pathogen virulence and are therefore under negative selection to maintain their function. Proof of concept of this idea was shown for the Hyaloperonospora arabidopsidis (Hpa) effector HaRxL96 and its homolog PsAvh163 from Phytophthora sojae. Both effectors suppress immunity in soybean and enhance susceptibility to virulent and avirulent Hpa when stably expressed in Arabidopsis. These findings demonstrate that evolutionarily conserved effectors from different oomycete species can have similar functions, even in non-host plant species [204]. The similar function of conserved effectors likely results from interaction with conserved plant proteins. Studying the plant proteins that are targeted by effectors can provide insights into the plant processes that effectors interfere with. 
An extensive interaction network of Arabidopsis proteins and pathogen effectors of different species was published in 2011 [205, 206]. The systematic yeast two-hybrid $(\mathrm{Y} 2 \mathrm{H})$ screens underlying this network resulted in the finding that 18 Arabidopsis proteins are targeted by effectors from multiple evolutionary distinct phytopathogens, including the oomycete Hpa [206]. The network provided a start for unravelling plant protein function and possible manipulation of plant processes by pathogen host-translocated effectors.

Here we set out to characterize the host-translocated RxLR and CRN repertoire of the spinach downy mildew Peronospora effusa (Pfs) and identify interacting plant proteins. Pfs is an obligate biotrophic oomycete that was first identified in 1824. Resistance-breaking isolates of the species have rapidly evolved over the last 3 decades following the introduction of new resistant spinach cultivars [30, 31, 32, 188]. The genome of the reference race 1 of $P f s$ encodes 99 candidate RxLR and 13 candidate $\mathrm{CRN}$ effectors. In a two-step approach we first classified the host-translocated effector repertoire based on their conservation with proteins of other oomycete species. This information was used to make a selection of interesting host-translocated effector proteins. In the second step we performed $\mathrm{Y} 2 \mathrm{H}$ interaction screens on 26 Pfs effectors to identify interacting plant proteins that could represent effector-targets. 


\section{Results and Discussion The Pfs 1 RxLR and CRN effector repertoire}

The predicted proteome of Pfs1 was previously mined for candidate RxLR and CRN host-translocated effectors using a regex script and HMM searches for WY folds and CRN motifs (Chapter 2). A total of 99 RxLR and $14 \mathrm{CRN}$-candidate effectors were identified by these criteria (Fig. 1). Note that one candidate effector, Pfs1|3487, has both a canonical RxLR and LFLAK motif and hence qualifies as RxLR as well as CRN candidate effector. The total number of proteins that are considered candidate effectors therefore totals 112 , not 113.

We found 64 secreted proteins with a canonical RxLR motif. Of these, 21 have WY domains and EER-like motifs, 7 have WY domains, and 13 have an EER motif. A total of 35 secreted proteins with degenerate

3 RxLR-like motifs ( $28 \times L R$ and $7 \mathrm{RxL}$ ) were found of which 28 lacked the first argenine ( $x L R)$ and 7 lacked the second $(R x L)$. Please note that these 35 proteins were selected because they contained additional domains commonly found in RXLR effectors; of them 17 have an EER-like motif, 17 have one or more WY domains and one protein has both (Fig. 1a). The genome of Pfs 1 has 5 canonical CRN effectors of which only two were recognized by the CRN HMM search. In addition, 6 secreted proteins with a degenerate LFLAK domain (3 L.LAK or 3 LF.AK) were found. Finally, 3 proteins were identified by the CRN HMM search as potential effectors and were hence included, despite lacking a recognizable translocation domain. Six of the candidate CRN effector proteins had an HVL motif (Fig. 1b). The inclusion of proteins that have degenerate translocation domains is justified because the exact requirements of translocation signals are still under debate. 
A

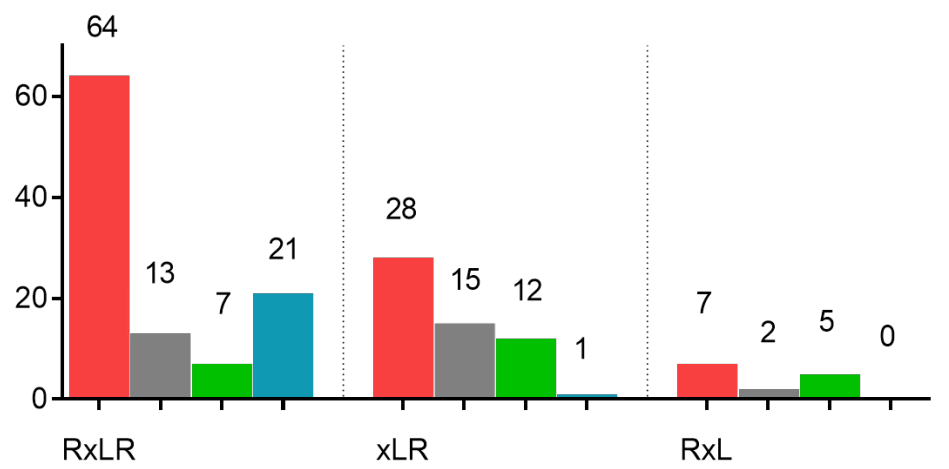

B

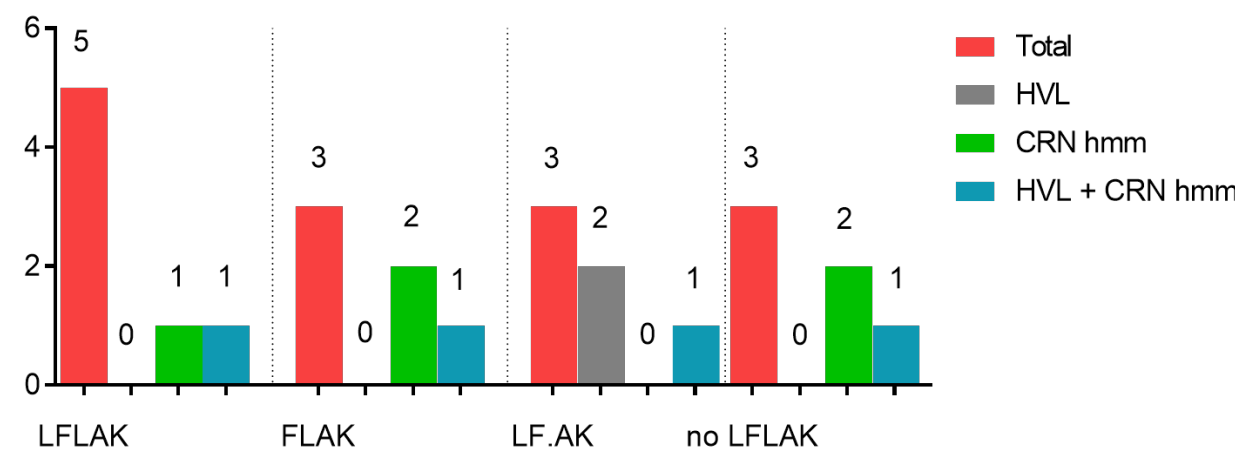

Figure 1. RxLR and CRN (-like) motifs observed in candidate host-translocated effectors identified in the genome of Pfs1. The candidate host-translocated effectors are grouped based on the variation in the putative translocation domain (RxLR or LFLAK). The number of proteins that have additional effector features are shown. For (degenerate) RxLR (A) the presence of a WY domain (green), EER-like motif (grey) or both (blue-green) is shown. For candidate CRN effectors the presence of an HVL (grey) motif and the recognition of the protein by the CRN HMM (green) or both (blue-green) are depicted.

\section{Secretome comparison identifies conserved and species-specific host-translocated effectors.}

The analysis of orthogoups (described in Chapter 2) identified orthology between Pfs proteins and proteins of other species. Here we use this data to study the conservation of host-translocated effectors of Pfs. Of the 112 effectors, 19 neither had orthologs (in other species) nor 
paralogs (within Pfs). These unique Pfs host-translocated effectors were therefore not placed in any orthogroup. The other 93 candidate effectors were assigned to 50 orthogroups of which 46 groups had RxLR effector candidates, 3 groups had CRN effector candidates and 1 group had both RxLR and CRN candidates. The latter has one predicted Pfs protein that qualifies as a RxLR as well as a CRN effector. One should note that also other proteins reside in these groups that do not all have the features of host-translocated-effectors, but nevertheless are orthologous.

We differentiated the orthogroups that have candidate Pfs hosttranslocated effectors into four classes; the Pfs-specific (not conserved), downy mildew-specific, Peronosporales-specific and broadly conserved orthogroups. The Pfs-specific effectors also include those that are not in any orthogroup at all. Of 112 Pfs host-translocated effectors 39 are Pfs-specific, 10 downy mildew-specific, 23 Peronosporales-specific, and 40 are more broadly conserved as they are in orthogroups that include proteins from Albugo and/or Pythium species (Fig. 2). In the next paragraphs these four different effector conservation classes are further discussed while highlighting some interesting orthogroups.

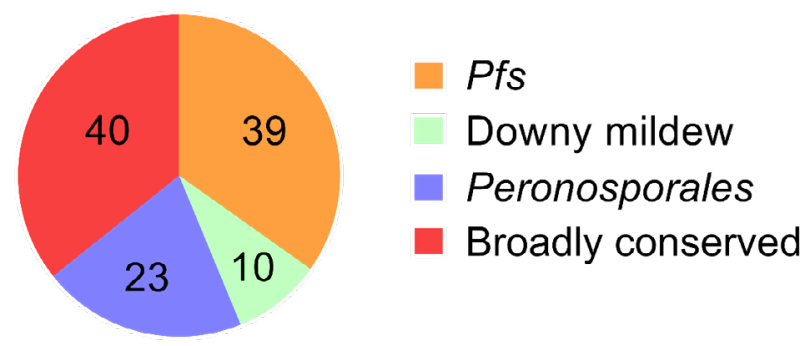

\section{Total $=112$}

Figure 2. Classification of the 112 candidate Pfs host-translocated effectors into four conservation classes.

Based on a comparative analysis including 18 oomycete species, the candidate hosttranslocated effectors of Pfs were classified as species-, downy mildew-, Peronosporalesspecific or broadly conserved. 
All of the 39 Pfs-specific candidate host-translocated effectors are RxLR proteins (Table S1). Nineteen are not related to any other protein within the proteome of Pfs1. The other 20 effectors were found in orthogroups that have between 3 and 17 paralogous Pfs proteins, both predicted effectors and other proteins. One interesting Pfs-specific effector group has eight proteins, of which seven are candidate RxLR effectors. Manual curation of the eighth protein identified an alternative gene model that has a signal peptide and is therefore also a candidate effector [207]. Polymorphisms, observed between Pfs races, in two of the candidate effectors in this group are associated with the breaking of resistance locus RFP2 [207].

The 10 downy mildew-specific host-translocated effectors are in 6 small orthogroups that have up to 12 proteins. Each group is only represented by two or three of the six downy mildew species represented in the study (Table 1). No Plasmopara proteins were found in these groups, which is in line with recent findings indicating that these species are not monophyletic with the other Hyaloperonospora/Peronospora downy mildew species [27]. In orthogroup OG0009188, the number of $P f s$ proteins is largely expanded. The orthogroup of twelve has eleven $P f s$ and one P. tabacina protein (Fig. 3). Three of the Pfs proteins are predicted to be secreted, of which only two are candidate effectors because the third one (Pfs1|10501) lacks a detectable RXLR sequence. Although the majority of proteins are not predicted to be secreted, 11 of them have WY-folds typical for RxLR effectors. Six of them have an additional degenerative RxLR motif. Manual curation led to an $\mathrm{N}$-terminal extension for only one protein, Pfs1|4091 (Suppl information 1). The extended protein has a signal peptide and combined with the $x L R$ and 3 WY domains, it is a candidate host-translocated effector. 


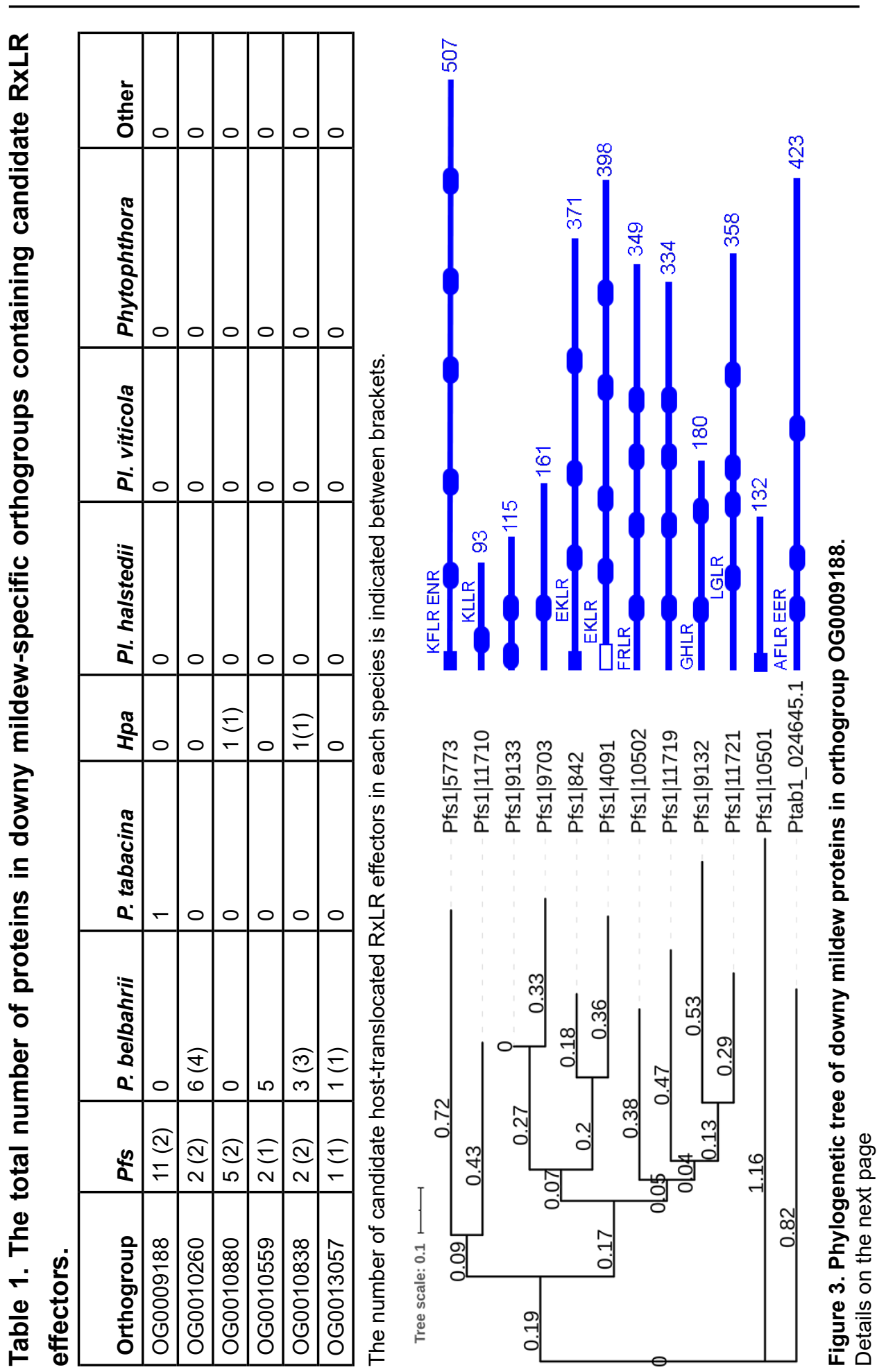


Figure 3. The tree includes a schematic representation of the protein showing the signal peptide (N-terminal square), RxLR like sequences and the WY domains (rounded squares) for each protein. Three proteins have a signal peptide ( $\mathrm{N}$-terminal filled square). Manual curation of Pfs1|4091 identified an additional start codon that yields a protein with a signal peptide (open square). Proteins are drawn to scale, except for the WY domain signs that are not drawn to scale but start at the accurate position. The number of amino acids is indicated at the C-terminus of the protein. Neighbor-Joining tree, Jukes-Cantor distance model. Number of substitutions per site are displayed at the branches).

The 23 candidate Peronosporales-specific RxLR effectors of Pfs grouped into 9 orthogroups. These groups contain proteins of both downy mildew and Phytophthora species, but not from more distantly-related species. Orthogroup OG0000009 is a major group that totals over 350 proteins, of which the numbers of downy mildew proteins $(1-35)$ are lower compared to that of Phytophthora species $(69-100)$. The group contains the PSR2 proteins of $P$. sojae and $P$. infestans that have WY domains, enhance virulence, and are known to suppress RNA silencing activity in plants $[95,110]$. PSR-effectors are a rare example of conserved host-translocated effector proteins in oomycetes. Pfs has 35 proteins in this group of which 14 are candidate effectors. All but two Pfs proteins have at least one WY-fold, a feature they share with the proteins of other species in this orthogroup. The protein alignment shows that only some residues in the WY-folds and their flanking amino acids are conserved between the proteins (Supporting information 1). Only $3(0.2 \%)$ of the amino acids in the alignment are identical in all proteins. The 21 Pfs 1 proteins that are not considered host-translocated effectors either lack a signal peptide (7) or RxLR motif (14).

Peronosporales-specific orthogroup OG0007491 is a group of 16 proteins that harbors HaRxL44, a known Hpa RxLR effector that was described to interact with Mediator Subunit 19a (MED19a) of Arabidopsis [91]. The interaction was found to result in the degradation of MED19a, leading to enhanced ethylene/jasmonic acid signaling and ultimately suppression of salicylic acid triggered immunity. The orthogroup contains two Pfs1 proteins that have duplicated within the Pfs genome. Recent duplication also occurred within the genomes of the other downy mildew species Peronospora belbahrii and Hpa (Fig. 4a). 
The reported RxLR-like motif 'RFL' of HaRxL44 is conserved in all proteins in this group except Pfs1|12209 ('RSL') [91]. Remarkably, the Hpa effectors have a downstream canonical RxLR motif that is accompanied by an EER(-like) motif in HaRxL44, HaRxL45 and HaRxLL108. The canonical RxLR motif and EER-like motif are not conserved between the Hpa and Pfs proteins (nor other proteins in the group, not shown) (Fig. 4b). To our knowledge, it has not been tested whether either or both of the motifs are required for translocation of HaRxL44.

Twelve of the orthologs in this group, including the Pfs proteins, have a predicted nuclear localization signal (NLS) (Fig. 4b). NLS are stretches of basic amino acids that are recognized and bound by importin- $\alpha$, a host protein that ultimately mediates the import into the nucleus [208]. Therefore, it is likely that the effectors target the host 3 nucleus, which was indeed confirmed for HaRxL44, 45 and HaRxLL108 $[209,210]$. 


\section{A}

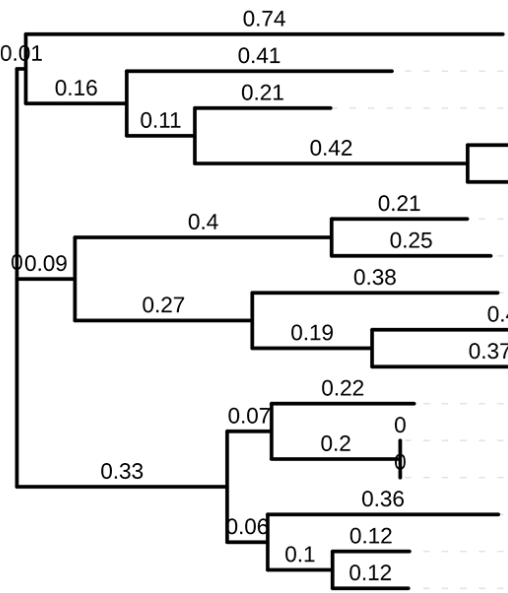

Hpa_HaRxL45

Hpa_HaRxL44

Hpa_HaRxLL108

Hpa_HaRxLL434

Hpa_HaRxL89

Pfs_Pfs1|12209-00001

Pfs_Pfs1|10382-00001

Pbe_PBEL 08345

Pbe_PBEL 02957

Pbe_PBEL 30210.1

PITG 04266T0

XP 008900182.1

XP 008913213.1

Pso_jgi Physo3 286166

XP 008916607.1

PITG 07586T0

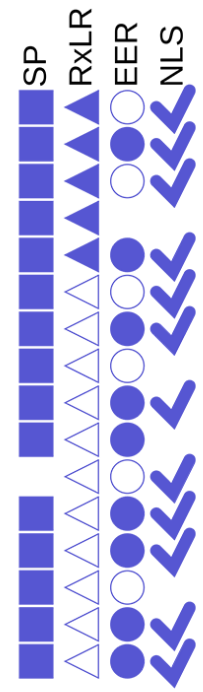

B
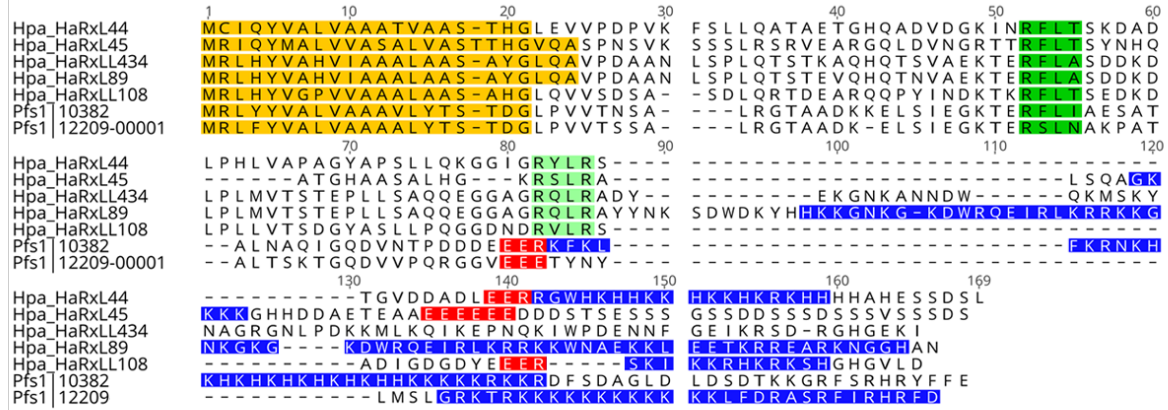

Figure 4. Phylogenetic tree of the Peronosporales-specific orthogroup OG0007491 of Hpa protein HaRxL44.

The majority of the proteins have a signal peptide (square) and a nuclear localization signal (checkmark). All proteins have a conserved RxLR-like ( $R x L)$ motif (open triangle). The Hpa proteins have an additional canonical RxLR mtoif (filled triangle). Most proteins have an EER or EER-like motif (closed vs. open circle). The alignment (b) of the Hpa and Pfs proteins shows that the RxL but not the RxLR motif is conserved between Hpa and $P f s$ proteins. The domains are highlighted in yellow (signal peptide), green ( $\mathrm{xL}$ motif), light green (RxLR motif) red (EER(-like)) motif and blue (basic amino acid residue-rich region contain a predicted NLS area). The Pfs proteins and HaRxL44 are marked bold. (Neighbor-Joining tree, Jukes-Cantor distance model. Number of substitutions per site are displayed at the branches). 
The final class, the broadly conserved effectors, has a total of 40 candidate effectors, that is all $13 \mathrm{CRN}$ and 27 of the RxLR proteins. These broadly conserved effectors are in large groups that also contain proteins from more distantly-related oomycetes, Pythium and Albugo, besides before mentioned downy mildew and Peronosporales species. The genomes of Pythium and Albugo species have small numbers of RxLRlike proteins and their contribution to virulence is not yet understood. A role in virulence is likely, because Albugo RxLR effectors were shown to suppress immunity and recently Pythium RxLR-like proteins were found to induce defense response in $N$. benthamiana [63, 64]. CRN effectors have evolved earlier in evolution and are known to exist within the order of Peronosporales as well as Albuginales and the Pythiales/Pythiaceae species [56]Seven of the thirteen candidate Pfs CRN effectors reside 3 in one orthogroup (OG0000033). The group has 234 proteins from Pfs, Hpa, Plasmopara, Phytophthora, Pythium and Albugo species. The other Peronospora species, Pta and Pbe are not represented in this group. The Pfs proteins are divided into four groups in the tree (Fig. 5). All 7 Pfs candidate CRN proteins are in one group (IV), together with 8 other Pfs proteins. No proteins of other species are present in this group, indicating that the expansion of these paralogs occurred within Pfs. Hpa, the closest relative of Pfs in this orthogroup, has one clade of 7 proteins that has specifically expanded within Hpa. The Hpa proteins are CRN homologs that have a RxLR motif (RELRLFLAK or a degenerative variant of that) [75]. The expansion of CRN proteins in downy mildew species is surprising because a number of CRN proteins are known to cause cell death, a condition that would be disadvantages for these obligate biotrophs [56]. 


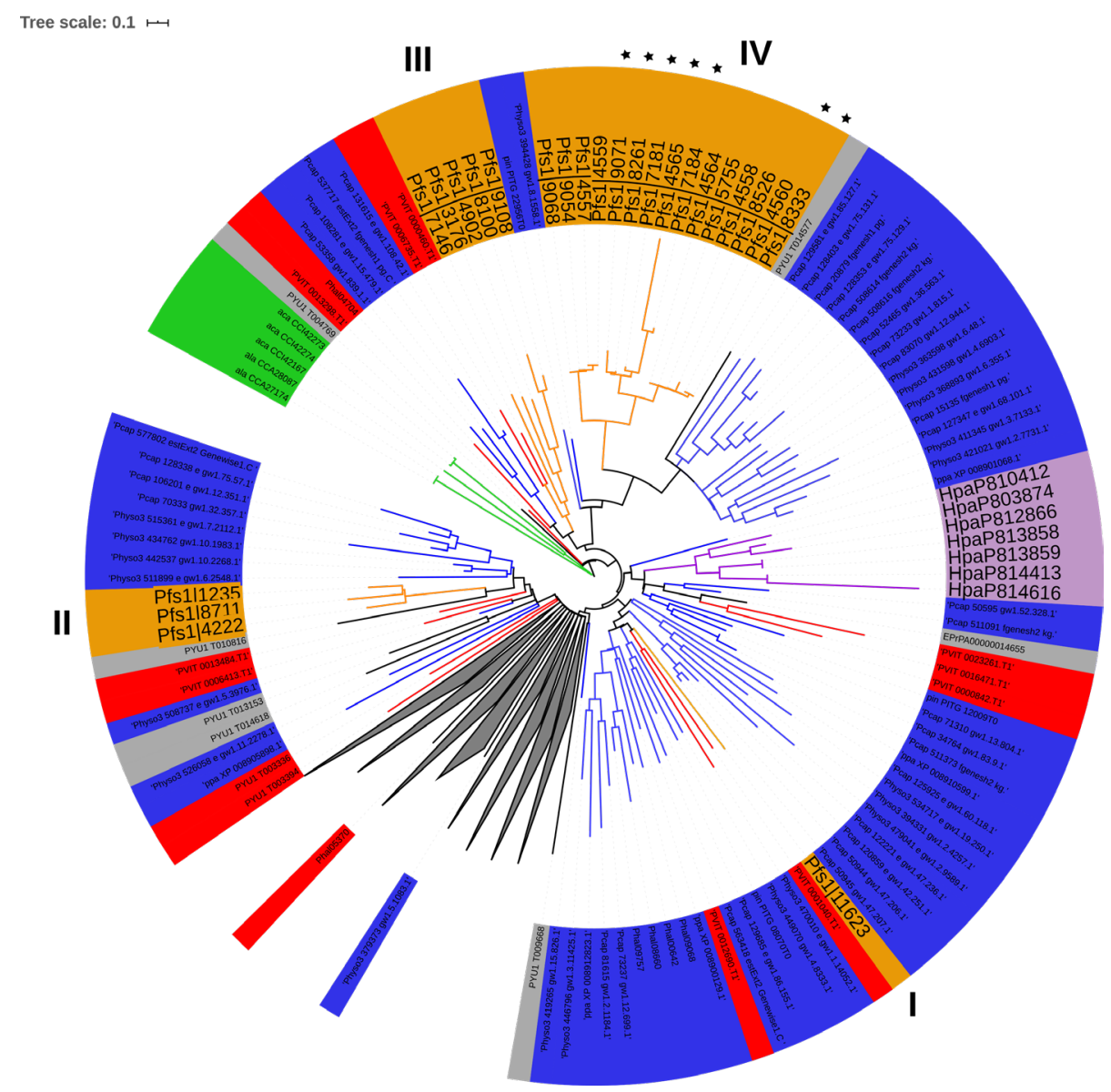

Figure 5. Rooted tree of the broadly conserved proteins of orthogroup OG0000033 that contains 7 candidate Pfs host-translocated CRN effectors. The total of 24 Pfs proteins (orange) are in four groups (I-IV). All 7 candidate hosttranslocated CRN effectors $\left({ }^{*}\right)$ are in clade IV that has expanded within Pfs. Eight clades without Pfs proteins (114 proteins) are collapsed. Phytophthora $=$ blue, Plasmopara $=$ red, $\mathrm{Hpa}=$ purple Pythium $=$ grey, Albugo $=$ green. The tree is created using DendroBLAST within Orthofinder and is rooted at the Albugo clade [211]. The tree was created using iTOL [212]. 


\section{Selection of candidate host-translocated effectors for interaction studies}

The challenge in effector biology is to understand how individual hosttranslocated effectors contribute to infection of the host. We used the orthology data to focus our research on a selection of host-translocated effectors that are interesting because of their conservation with other species and the presence of canonical translocation motifs. In total, 40 candidate host-translocated effector proteins of Pfs1 were selected for functional analysis (Table S2). Of them, 6 are Pfs-specific, 4 only occur in downy mildew species, 14 are conserved within the Peronosporales and 17 are broadly conserved. The selected proteins include $28 \mathrm{RxLR}, 2 \mathrm{CRN}$ effectors and Pfs $1 \mid 3487$ that has both a RxLR and CRN translocation domain. In addition, we selected $2 \mathrm{CRN}$-like proteins without a signal

3 peptide, 4 candidate host-translocated effectors that were predicted from previous Pfs 1 genome assemblies and one protein with degenerate RxLR and CRN motifs but no other effector features. Finally, 4 proteins were selected because they are in orthogroups with selected candidate Pfs host-translocated effectors. These proteins have multiple WY domains but lacked a RxLR(-like motif). From now on, all above mentioned proteins will be referred to as 'effector baits'.

\section{Yeast two-hybrid screening identifies Pfs effector-interacting plant proteins}

The effector baits were amplified from mRNA derived from Pfs1-infected spinach plants. Effector baits were cloned, without the signal peptide coding sequence, into the gateway pDEST22 vector for expression in yeast. In total, 33 effector bait sequences were successfully cloned and transformed into yeast. None of the candidate effector-fusions autoactivated the HIS reporter gene in the absence of a prey protein. The effector-constructs were used as baits in a yeast two-hybrid screen with a prey library of oligo-dT primed cDNA of Arabidopsis.

Out of 33 cloned effector baits, 26 were successfully mated in the yeast two-hybrid screens. For the six baits that were not mated 
successfully the required number of colonies ( $>1$ million) to be screened was not sufficient. The effector baits usually had highly-specific interactions with plant proteins. For eight effector baits only one plant interactor was found, while 10 interacted with multiple unique plant proteins. Four plant proteins were found to interact with multiple effector baits. For 9 effector baits, no interactors were obtained from the screen.

Selected bait and prey vectors were retransformed into yeast to verify their interaction. A total of 40 unique plant proteins (51 unique colonies) were found to interact with 18 effector candidates (Table 2 and Table 3). The identification of plant proteins that interact with Pfs effectors provide a first insight into the possible virulence targets of $P f s$. Importantly, these results need to be verified in planta, to assess the biological relevance. Within the $\mathrm{Y} 2 \mathrm{H}$ system, both bait and prey proteins are directed to the nucleus, so it is possible to identify interactions between proteins that are spatially separated under natural conditions [213]. The further verification of the subcellular localization of $P f s$ effectors and their interacting plant proteins is further discussed in chapter 4. 
Table 2. Number of interacting plant proteins per candidate Pfs effector detected in $\mathrm{Y} 2 \mathrm{H}$ screening (ranked by number of plant interactors).

\begin{tabular}{|c|c|c|c|c|}
\hline Effector & Motifs and folds & Conservation & Orthogroup & $\begin{array}{l}\# \text { of plan } \\
\text { interactors }\end{array}$ \\
\hline Pfs1|10492 & RxLR EER WY & Peronosporales & OG0000009 & 8 \\
\hline Pfs1|6786 & RXLR EER WY & Pfs-specific & OG0010381 & 7 \\
\hline Pfs $1 \mid 1800$ & RxLR EER & Peronosporales & OG0007294 & 5 \\
\hline Pfs1|3869 & RxLR & Downy mildew & OG0010260 & 5 \\
\hline Pfs1|9071 & LFLAK & Broad & OG0000033 & 5 \\
\hline Pfs $1 \mid 3172$ & RxLR EER TM & Downy mildew & OG0010838 & 3 \\
\hline Pfs $1 \mid 3210$ & xLR EER & Peronosporales & OG0006720 & 3 \\
\hline Pfs1|7215 & RxLR & Broad & OG0000021 & 3 \\
\hline Pfs1|3487 & RxLR LFLAK HVL & Broad & OG0000437 & 2 \\
\hline Pfs $1 \mid 4005^{*}$ & LFLAK & Broad & OG0000657 & 2 \\
\hline Pfs1|10453 & RxLR EER & Broad & OG0000023 & 1 \\
\hline Pfs $1 \mid 10763^{*}$ & RxLR EER WY & Pfs-specific & OG0007292 & 1 \\
\hline Pfs1|2936 & RxLR & Broad & OG0000062 & 1 \\
\hline Pfs1|3069 & xLR WY & Peronosporales & OG0000009 & 1 \\
\hline Pfs $1 \mid 805$ & RxLR WY & Peronosporales & OG0000009 & 1 \\
\hline Pfs $1 \mid 8598$ & xLR EER & Broad & OG0003089 & 1 \\
\hline Pfs1|9976 & LF.AK HVL & Broad & OG0000222 & 1 \\
\hline TR14313** & RxLR EER WY & Downy mildew & OG0001021 & 1 \\
\hline Pfs1|10418 & $x L R$ & Broad & OG0000255 & 0 \\
\hline Pfs1|129 & WY no RxLR & Peronosporales & OG0000009 & 0 \\
\hline Pfs1|4263 & RXLR EER WY & Pfs-specific & OG0010381 & 0 \\
\hline Pfs $1 \mid 9414$ & RxLR EER WY & Pfs-specific & OG0033384 & 0 \\
\hline Pfs $1 \mid 964^{*}$ & LFLAK & Broad & OG0000723 & 0 \\
\hline Pfs1|9237 & RxLR EER WY & Pfs-specific & OG0007292 & 0 \\
\hline Pfs $1 \mid 8028$ & RXLR EER WY & Peronosporales & OG0000009 & 0 \\
\hline Pfs1|8288a & RxLR WY & Peronosporales & OG0000009 & 0 \\
\hline Pfs $1 \mid 8804$ & RxLR EER & Broad & OG0004556 & 0 \\
\hline Pfs1|1192 & RxLR EER WY & Peronosporales & OG0000009 & ND \\
\hline Pfs1|12059 & RxLR WY & Peronosporales & OG0000009 & ND \\
\hline Pfs1|13089 & RXLR EER WY & Peronosporales & OG0000009 & ND \\
\hline Pfs1|8208a & RXLR EER WY & Peronosporales & OG0010393 & ND \\
\hline Pfs $1 \mid 8804$ & RxLR EER & broad & OG0004556 & ND \\
\hline Pfs $1 \mid 8905^{*}$ & RXLR EER WY & Pfs specific & OG0007292 & ND \\
\hline
\end{tabular}

Asterisks (*) mark proteins without signal peptides. 'EER' indicates EER-like motifs, TM is a transmembrane domain and '.' is any amino acid accept leucine (L). **The full name of TR14313 is TR14313|c0_g2_i1|m.24305-00001. For proteins marked 'ND' (not done) no successful mating was performed. 


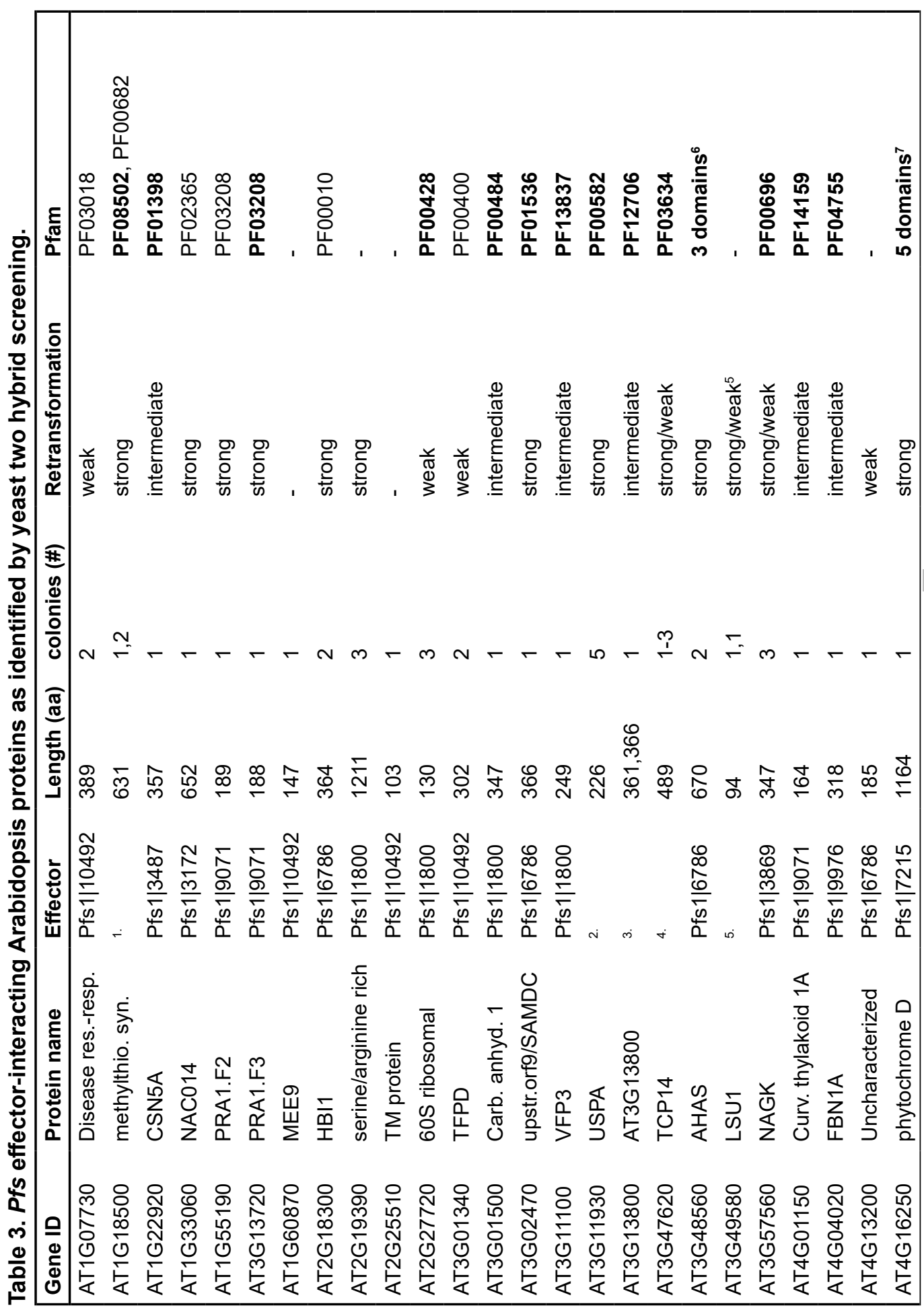



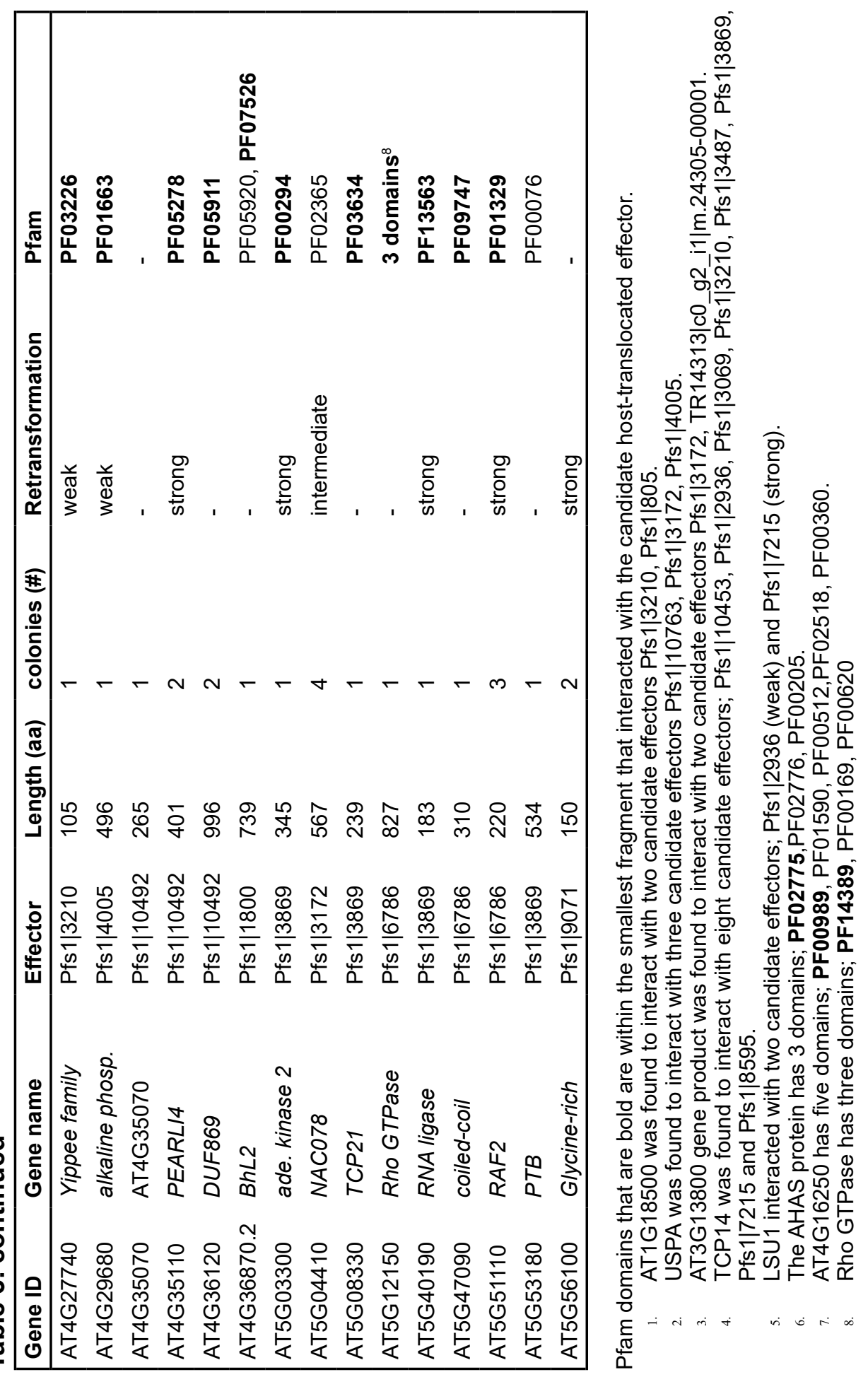


\section{Interaction network of Arabidopsis proteins targeted by host-translocated Pfs effectors}

A public database, EffectorK [214] was mined for experimentally-verified interactions between the Arabidopsis proteins identified in the Pfs effector $\mathrm{Y} 2 \mathrm{H}$ screens and other proteins. The EffectorK interaction dataset includes experimentally-confirmed protein-protein interactions from 2035 peer-reviewed publications and contains Arabidopsis-Arabidopsis as well as Arabidopsis-effector protein interactions. Effectors from bacterial (Pseudomonas, Agrobacterium, Xanthomonas, Ralstonia), fungal (Golovinomyces) and oomycete ( $H$. arabidopsidis) phytopathogens are represented [205, 206, 214, 215, 216, 217, 218]. The Pfs effector interactome supplemented with the published interactions was visualized in a Pfs interaction network (Fig. 6).

We obtained 1220 literature curated interactions between the Pfs

effector-interactors and other proteins from the database, of which 1089 with plant proteins and 131 with effectors of other pathogen species. The interactions between proteins can give an idea about the function of Pfs effector-interactors in the plant. Possibly the host-translocated effectors interfere with this function.

Previous studies suggested that effectors, of a wide range of pathogens, target similar plant proteins [206]. These proteins are characterized by their high connectivity within the protein interaction network [219]. The Pfs $\mathrm{Y} 2 \mathrm{H}$ interaction network is centered around six 'hubs' of highly connected proteins; low sulfur upregulated 1 (LSU1), TEOSINTE BRANCHED, CYCLOIDEA AND PROLIFERATING CELL FACTORS 14 (TCP14), virF interacting protein 3, (VFP3), constitutive photomorphogenesis 5A (CSN5A), fibrillin, and Prenylated Rab acceptor 1 (PRA1). Proteins in each of these hubs are targeted by effectors of other pathogens as well. 


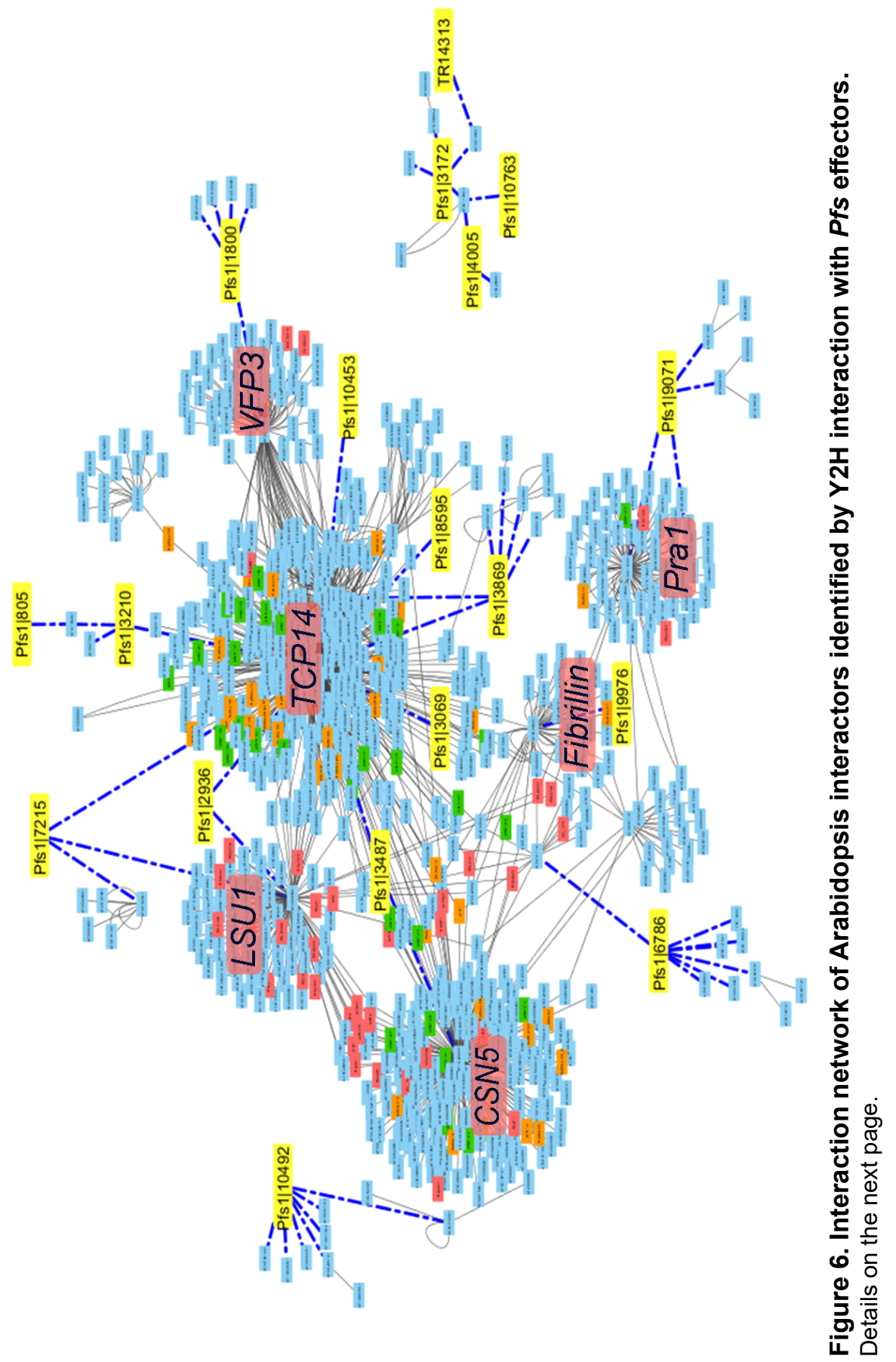




\section{Figure 6 continued}

The network includes published interactions between Arabidopsis (blue) Pfs (yellow), Hpa (orange), fungal (Golovinomyces, green) and bacterial (Pseudomonas, Agrobacterium, Xanthomonas, Ralstonia, red) effectors. The interactions identified in this study are indicated with dashed blue lines and the highly connected proteins are highlighted (light red box). $L S U 1=$ low sulfur upregulated $1, T C P 14=$ TEOSINTE BRANCHED, CYCLOIDEA AND PROLIFERATING CELL FACTORS 14, VFP3 virF interacting protein 3, Pra1 $=$ prenylated Rab acceptor $1, F B N 1 A=$ Fibrillin precursor protein $1 \mathrm{~A}, C S N 5 A=$ constitutive photomorphogenesis $5 \mathrm{~A}$.

Nine of the Pfs1 effector protein-interactors identified in the $\mathrm{Y} 2 \mathrm{H}$ screens were previously described to interact with effectors from other species (Table 4). CSN5A, PRA1.F3 (Prenylated Rab acceptor 1.F3), TCP14 and fibrillin 1a (FBN1a) even interact with effectors from species of three kingdoms of life represented in the database. VFP3 and LSU1 are targeted by bacterial and Pfs effectors. These nine candidate effector proteins are highly connected within the overall Arabidopsis interactome and are likely important regulators of cellular processes and possibly linked to plant immunity or other aspects of the infection process. 


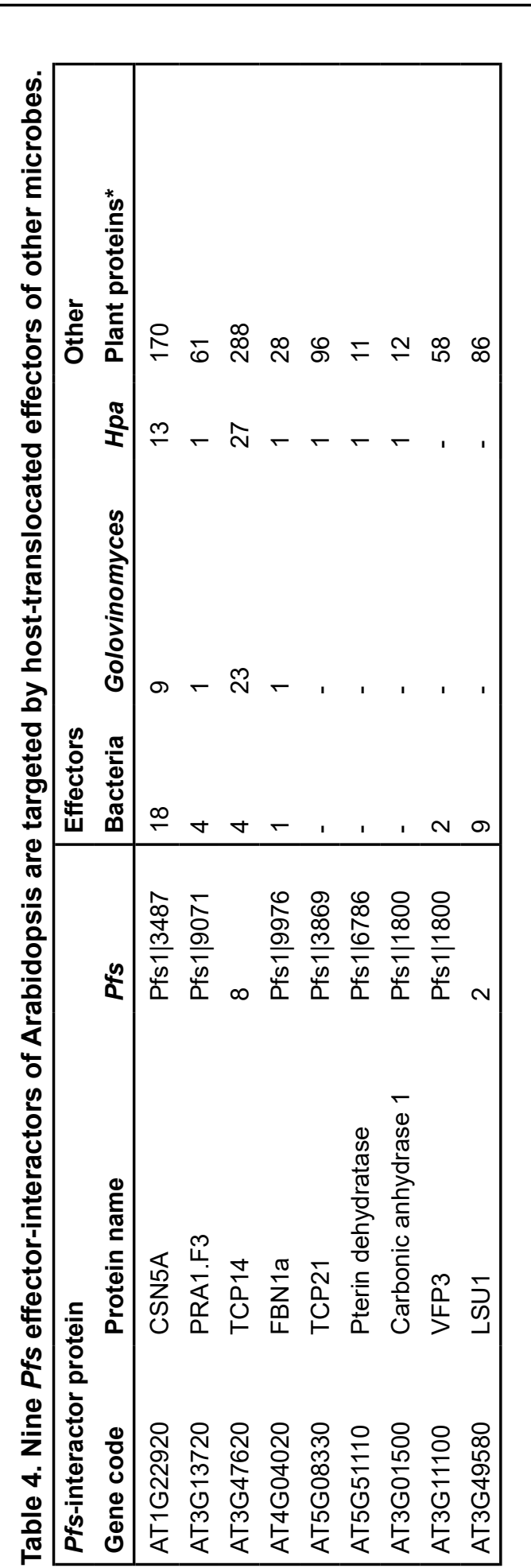

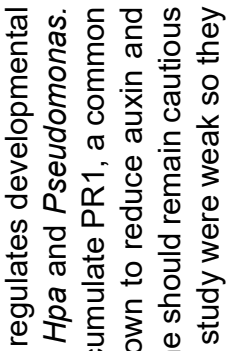

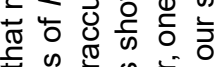

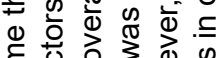

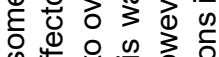
응 웡 웅 은 은

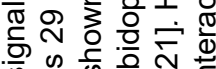

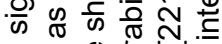

\section{3}



गั

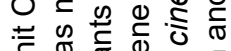

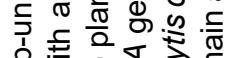
की कूष

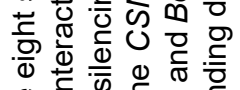
d.$\subseteq \frac{1}{5}$ प응 को 는

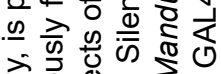
ㅎํㄹ원

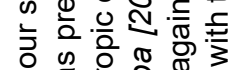

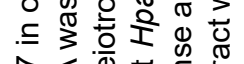

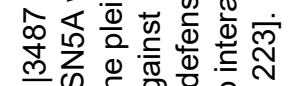
Бٓ


ब.

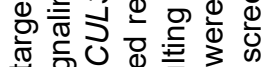

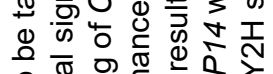

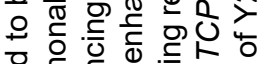

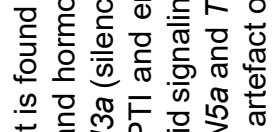

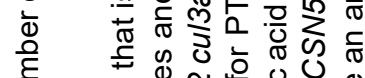
ᄃ

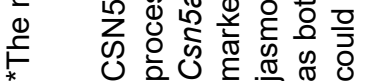


The majority of the Pfs effector-interacting proteins were less connected in our network. As many as 27 Arabidopsis interactors have between one and five connections with other proteins. Six of these lesser connected host-translocated effectors are connected to the bigger 'hubs' by one of their interactors but also have interactors that are not linked to the hubs. An interesting example is host-translocated effector Pfs $1 \mid 6768$ that is linked to the fibrillin centered hub of the network through its interaction with Rubisco accumulation factor 2 (RAF2, AT5G51110). RAF2 is required for Rubisco biogenesis, its link to fibrillin is unknown [224] Of the other six interactors of Pfs $1 \mid 6786$, five do not interact with any protein other than Pfs1|6786. Remarkably, gene expression of five of the Pfs1|6786 interacting protein encoding genes, including RAF2, were reported to be down-regulated upon treatment of Arabidopsis plants with salicylic acid (SA), suggesting a response to immune signaling [225]. The interacting proteins include transcriptional regulators like the negative regulator of defense BhLh64/HB/1 (AT2G18300) [226], a coiled-coil protein of unknown function (AT5G47090) and an upstream open reading frame that encodes a peptide that regulates SAMDC1 transcription [227, 228]. Overexpression of $S A M D C 1$ was shown to elevate spermine levels and induce defense-related gene expression and higher resistance to Pseudomonas syringae and Hpa [229]. The other PFS1|6786 interactorproteins are involved in metabolic processes like amino acid synthesis (acetolactate synthase (AHAS), AT3G48560) or have no known functions (AT4G13200, AT5G12150) [225, 230, 231].

Although the function of the Pfs1/6786 interactors are reasonably well described, the function of the majority of lesser connected Pfs interactor-proteins is unknown. For instance, Pfs1|10492 was found to interact with seven Arabidopsis proteins. The proteins are not reported to interact with each other and for five of them, their molecular function is unknown. One of the two proteins that have been more extensively studied is Pfs effector-interactor protein AT3G01340. It interacts with its paralog AT2G30050 that links this effector to the CSN5A signaling hub of the network. AT3G01340 and AT2G30050 are the paralogs of the budding 
yeast Sec13 protein that mediates the formation of COPII coated vesicles that transport proteins from the ER to the Golgi system [232]. The other studied interactor AT2G25510 does not have a known molecular function but was associated with defense against $H$. arabidopsidis because of its high expression levels in Arabidopsis downy mildew resistant 5 and 6 (dmr5 and dmr6 ) mutants identified in a ethyl methane sulfonate (EMS) screen [233].

Other Pfs host-translocated effectors with a link to a large hub in the network and additional independent interactors are Pfs1|9071, Pfs1|3869, Pfs1|1800. The low complexity interaction network of these proteins makes them interesting targets for further functional analysis as their function may be easier to comprehend than that of highly connected proteins.

Four candidate host-translocated effectors are not connected to the main network at all. They from a separate cluster because Pfs $1 \mid 3172$ shares interactor proteins with the other three host-translocated effectors. Pfs1|4005, Pfs1|10763 and Pfs1|3172 all target AT3G11930, a protein that has a universal stress protein domain A (USPA) [234]. This domain is omnipresent in both prokaryotes and eukaryotes and is thought the be involved in the response to environmental stimuli. The network shows that it interacts with AT2G47710, that has a USPA domain as well. The protein is targeted by three Pfs host-translocated effectors but not any of the other effectors in the network, so it may be a Pfs-specific interactor target. Pfs1|3172 and TR14313|c0_g2_i1|m.24305-00001 both interacted with AT3G13800 in the Y2H screen. AT3G13800 has a Metallo-hydrolase/ oxidoreductase superfamily protein domain but its molecular function is otherwise unknown. Besides the shared Arabidopsis interactor proteins, Pfs1|3271 interacts with two NAC transcription factors, NAC014 and NAC078. Both NACs have a C-terminal transmembrane domain, just like Pfs1|3172. NACs in this family have been shown to be effector targets of $P$. infestans, Hpa and Bremia lactucae effectors [86, 169, 206]. Their possible function in immunity will be discussed in chapter 4 . 


\section{Prioritizing plant interactors for further validation}

The $\mathrm{Y} 2 \mathrm{H}$-based effector interaction network provides a first insight into the cellular processes that may be targeted by host-translocated Pfs effectors. Further research is needed to understand the role of the effectors and their targets in the infection process. We used the $\mathrm{Y} 2 \mathrm{H}$ and interaction network to select fourteen interactors, targeted by eight candidate effectors, that are of particular interest for further characterization (Table 5). In the selection we prioritized $\mathrm{Y} 2 \mathrm{H}$ interactors that were represented by multiple unique gene fragments and/or showed strong interaction with the effector upon retransformation, over weaker interactors represented by single fragments. Interactions between multiple unique fragments and an effector protein served as independent replicas of the interaction. We selected nine effector-interactors that had less than ten connections in the network. Five other interactors had between fourteen and 110 connections but could be interesting because they have been published to interact with effectors of other species or are known to affect disease susceptibility. The further characterization of these fourteen high confidence interactors and their eight effector proteins is the topic of chapter $\mathbf{4}$ of this thesis. 
Chapter 3

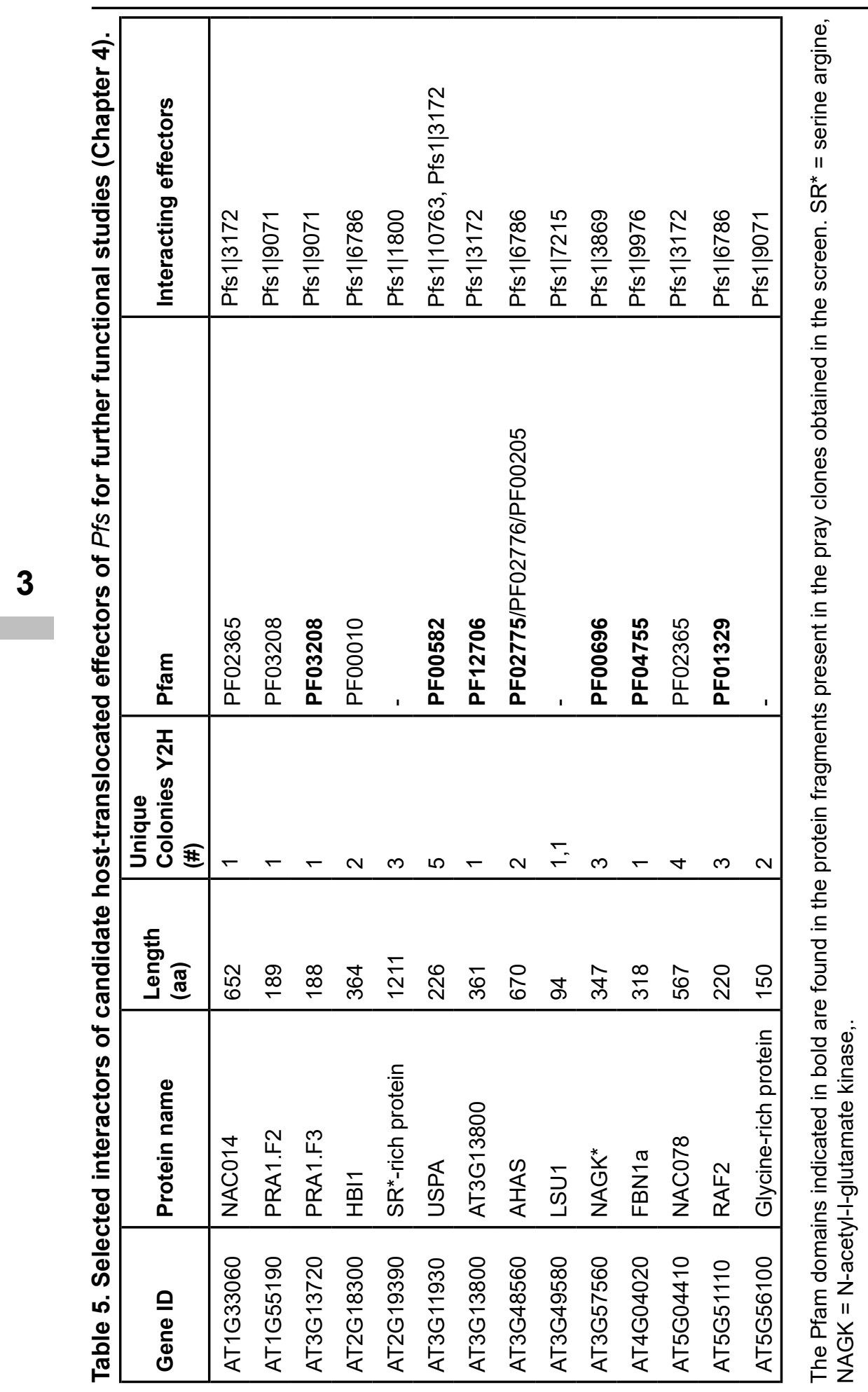




\section{Conclusion}

Comparative analysis of the candidate RxLR and CRN effectors of $P f s 1$ revealed that 39 effectors are Pfs specific, 10 are downy mildew specific, 23 are Peronosporales specific and 40 more broadly conserved. Candidate effector Pfs1|10382 is in an orthogroups with $H$. arabidopsis effector HaRxL44 and possibly has a similar function. Using $\mathrm{Y} 2 \mathrm{H}$ assays, 40 Arabidopsis proteins that interact with 26 Pfs candidate effectors were identified, to our knowledge representing the first known plant interactors of $P f s$ effectors. The interaction data were supplemented with published interactions with other proteins, providing an interaction network that gave insight into connectivity and possible processes in which the plant interactors may be involved. Based on the interaction strength in the $\mathrm{Y} 2 \mathrm{H}$ assays and the information in the interaction network, fourteen high confidence plant interactors and eight corresponding Pfs 1 were selected to study their interaction in planta.

\section{Material and Methods Computational analysis of secreted proteins for comparative genomics}

The clustering of orthologous proteins using Orthofinder, the secretome prediction and the effector identification were described in chapter 2. The proteome of Pfs was compared to that of four downy mildew, four Phytophthora, two Albugo and 5 Pythium species (Chapter 2, Table S1).

\section{Selection of candidate host-translocated effectors for functional validation}

Candidate host-translocated effectors were prioritized based on their conservational status and the presence of known motifs and domains in their amino acid sequence. Candidate host-translocated effectors that are conserved between downy mildew or Peronosporales species and canonical effectors were prioritized over candidate host-translocated effectors that were unique to Pfs or had degenerative motifs. Three 
additional candidate host-translocated effectors were identified by manual annotation of genes in the final assembly of $P f s 1$ (Supplemental data 1).

\section{Yeast-two hybrid library construction}

An oligo-dT primed Arabidopsis thaliana Col-0 cDNA library was produced using Invitrogen Custom Services (Invitrogen, Carlsbad, California). Ten day old seedlings were exposed to five different treatments; (1) infection with $H$. arabidopsidis Cala2 (incompatible, harvested 3 days post infection (dpi)) or (2) $\mathrm{H}$. arabidopsidis Noco2 (compatible, (3 dpi)), (3) treatment with BTH (24 hours, sprayed with $0,1 \mathrm{mg} / \mu \mathrm{l}),(4)$ infiltration with the NLP3-derived peptide pattern nlp24 (24 hours), or (5) untreated. Plant material was harvested for RNA isolation using phenol/chloroform extraction. RNA samples were mixed at a 1:1:1:1:1 ratio, using $2 \mathrm{mg}$ of

RNA for each sample. A three frame uncut cDNA library was made using oligo-dT primers and cloned into pENTR22 entry vectors using Gateway technology [235]. Cloning of the cDNA library resulted in $168 \times 10^{6}$ colony forming units with an average insert size of $1.5 \mathrm{~kb}$. The inserts were then recombined into $\mathrm{Y} 2 \mathrm{H}$ prey vector pDEST22 to create GAL4 activation domain fusions. Transformation into $E$. coli $\mathrm{DH} 10 \mathrm{~B}$ resulted in $7.9 \times 10^{6}$ colony forming units with an average insert size of $1.55 \mathrm{~kb}$.

\section{Yeast-two hybrid bait construction}

Peronospora effusa reference race 1 (Pfs1) was provided by the Dutch breeding company Rijk Zwaan Breeding BV in 2014. The growth conditions and infection protocol are described in chapter 2. At 4, 5, 6 and 7 days post infection leaf samples were harvested for mRNA isolation using the Qiagen mRNA isolation kit. cDNA was generated using oligo-dT primers at standard settings. Effector coding sequences were amplified from the predicted signal peptide cleavage site (SignalP v4.0, setting 0.34). A start codon was added to the forward primer (Table S3). The effectors were recombined into the $\mathrm{Y} 2 \mathrm{H}$ bait vector pDEST32 to create DNA-binding domain (DBD) effector protein fusions. All entry clones were verified by Sanger sequencing. The insert size of the destination vectors was confirmed using colony PCR. 


\section{Yeast strains and transformation}

The bait and prey vectors in this study were transformed into yeast strain Y8800 and Y9300, respectively. To make competent yeast cells, yeast was grown overnight in $250 \mathrm{ml}$ of YEPD at $20^{\circ} \mathrm{C}$ while shaking. The medium was transferred to a $28^{\circ} \mathrm{C}$ shaking incubator and grown until the $\mathrm{OD}_{600}$ was between 0.2 and 0.8 . The yeast cells were collected by centrifuging the medium at $1800 \mathrm{rpm}$ for five minutes. The YEPD was discarded and

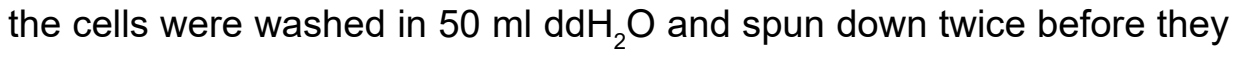
were dissolved in 50ml TE/LiAc (10 mM Tris, $1 \mathrm{mM}$ EDTA, $100 \mathrm{mM}$ LiAc). After a final centrifugation, the now competent cells were dissolved into $\mathrm{TE} / \mathrm{LiAc}$ to a final $\mathrm{OD}_{600}$ of 50 . For a single transformation reaction, $20 \mu \mathrm{l}$ of competent yeast cells was added to a premixed reaction mix of $82 \mu \mathrm{l}$ $60 \%$ PEG, $11 \mu \mathrm{l}$ of $10 \times \mathrm{TE}, 13 \mu \mathrm{l} 1 \mathrm{M} \mathrm{LiAc}, 20 \mu \mathrm{l}$ of salmon sperm DNA and $200 \mathrm{ng}$ of plasmid DNA. The salmon sperm DNA was heated at $95^{\circ} \mathrm{C}$ for five minutes and kept on ice prior to use. Transformation reactions were incubated at $30^{\circ} \mathrm{C}$ for 30 minutes and transformed to a $42^{\circ} \mathrm{C}$ water bath for 15 minutes. One $\mathrm{ml}$ of $\mathrm{ddH}_{2} \mathrm{O}$ was added to each tube and the cells were spun down at $5000 \mathrm{rpm}$ for 30 seconds to collect the yeast. The yeast pellet was resuspended in $\mathrm{dd}_{2} \mathrm{O}$ and plated on synthetic complete (Sc) plates with the appropriate marker (Sc-Trp for bait and Sc-Leu for prey). Plates were incubated at $30^{\circ} \mathrm{C}$ and colonies would be visible after three days.

The library transformation was done in a similar fashion, just increasing the volume of the competent cells to $9.6 \mathrm{ml}$ and the other components of the reaction to ratio. A total of $121,8 \mu \mathrm{g}$ of library DNA was used. The medium was plated on Sc -Leu-Trp plates to select for cells that obtained the bait as well as a prey vector. The transformation yielded $>1 \times 10^{6}$ colony forming units (cfu's) The yeast strains were harvested in YEPD $+20 \%$ glycerol. $1 \mathrm{ml}$ aliquots of $\mathrm{OD}_{600} 40$ were stored at $-80^{\circ} \mathrm{C}$. 


\section{Library screen}

The library screen was performed using the mating method [236]. A yeast library aliquot of $1 \mathrm{ml}$ was thawed on ice, transferred to $100 \mathrm{ml}$ YEPD and incubated on a shaker at $28^{\circ} \mathrm{C}$ for one hour. Cells were centrifuged at 1800 rpm for 5 minutes and washed in $\mathrm{ddH}_{2} \mathrm{O}$ twice. Cells were resuspended in YEPD to $O D_{600}=3$.

The pDEST32 bait vectors with effector proteins were grown overnight in $20 \mathrm{ml}$ of YEPD with ampicillin in $50 \mathrm{ml}$ Falcon tubes while shaking at $20^{\circ} \mathrm{C}$ until they reached the exponential growth stage $(0.2-$ $0.8 \mathrm{OD}$ ) and collected by centrifugation as described above.

For each mating reaction $3 \mathrm{ml}$ of library and $6 \mathrm{OD}$ (so, in case of $\mathrm{OD}_{600}=0.5,12 \mathrm{ml}$ is used.) of bait transformed yeast were mixed, centrifuged, resuspended in $300 \mu$ of $\mathrm{ddH}_{2} \mathrm{O}$ and plated on a YEPD (+

$3100 \mu \mathrm{g} / \mathrm{ml}$ ampicillin) plate. After 4 hours of incubation at $30^{\circ} \mathrm{C}$ yeast was collected from the plates, suspended in $\mathrm{ddH}_{2} \mathrm{O}$ and collected by centrifugation. The cells were resuspended in $600 \mu \mathrm{lddH}_{2} \mathrm{O}$ and plated on synthetic complete (SC) plates without leucine (Leu) tryptophan (Trp) and histidine (His) supplemented with ampicillin (Amp). A 1:10,000 dilution was plated onto SC - Leu - Trp to monitor the number of diploid colonies screened. A minimum of 1 million colonies was screened for each bait. After four days of incubation the colonies were picked from the Sc -Leu -Trp -His plates and suspended in $15 \mu \mathrm{ddH}_{2} 0$. The dissolved colonies were than spotted on two fresh SC -Leu -Trp -His. One plate was used for replica plating onto SC -Leu -Trp - His plates supplemented with 2 or $5 \mathrm{mM}$ of the histidine biosynthesis inhibitor 3-amonio-1,2,4 -triazole (3-AT) (ForMedium) to test the strength of the interaction. Plates were incubated for two days and pictures were taken to document the growth of the yeast.

The other plate was used for colony PCR. Yeast colonies were lightly picked and transferred to $30 \mu \mathrm{l}$ of $0.02 \mathrm{M} \mathrm{NaOH}$. The samples were heated $\left(99^{\circ} \mathrm{C}, 10\right.$ minutes), cells were collected using centrifugation and $1 \mu$ of the supernatant was used for colony PCR. The colony PCR reactions were $10 \mu \mathrm{l}$ each and DNA was amplified using 
Dreamtaq DNA polymerase (ThermoScientific) and primers for pDEST22 (Forward; TATAACGCGTTTGGAATCACT, Reverse; ACCTTGATTGGAGACTTGAC). Prey PCR products were purified (Agencourt AMPure XP beads) and Sanger sequenced.

Sequences were analyzed using Geneious $v$ 9. Arabidopsis coding sequences that were out of frame with the GAL4-AD domain or where less than 50 amino acids in length were discarded. The corresponding full-length Arabidopsis genes were identified by finding the best hit in the NCBI non-redundant database using BlastN.

For every identified gene a representative colony was grown in $3 \mathrm{ml}$ of YEPD. To isolate the plasmids the culture was centrifuged (2000 rpm, 2 minutes), resuspended in $1 \mathrm{ml}$ of TE (10 mM Tris-HCl, 1 mM EDTA, $\mathrm{pH}$ 7.5). After another round of centrifugation, the cells were suspended in $200 \mu \mathrm{l}$ Resuspension buffer with RNAse A (Macherey Nagel NucleoSpin Plasmid EasyPure, Mini kit for easy plasmid DNA purification), $15 \mathrm{U}$ of Zymolase-20T (Amsbio \#120491-1) and $2 \mu \mathrm{l}$ of 2-Mercaptoethanol. Samples were incubated at $37^{\circ} \mathrm{C}$ for an hour before plasmid isolation with the GenElute plasmid miniprep kit (Sigma). Plasmids were amplified via transformation into DH5a cells. The obtained plasmid DNA (from a single colony) were used in the retransformation assay.

The interaction between bait and prey was verified by transforming both plasmids into yeast strain Y8930. Colonies were selected on SC -Leu -Trp - His plates and replica plated on SC -Leu -Trp -His + 2 and 5 mM 3-AT.

\section{Network analysis}

All datasets from the newly published EffectorK database were downloaded and mined for interactions that involved the Arabidopsis proteins that interacted with the Pfs effector baits in this study [214]. A network was created using cytoscape v3.7.9. and improved using Photoshop v1.9.1 to highlight the more connected interactors. 


\section{Acknowledgements}

We thank Ronnie de Jonge for his useful input for the orthology analysis, Pim Vergeer for creating the Arabidopsis yeast two-hybrid library and Alexandra Pelgrom for her help during the transformation of the library and her expertise in yeast mating. Additionally, we thank TKI T\&U (Topsector Tuinbouw \& Uitgangsmaterialen), Enza Zaden, Rijk Zwaan, Syngenta and Pop Vriend for funding the project.

\section{Supplemental Tables.}

\section{Table S1. RxLR and CRN effectors of Pfs1}

Table $\mathrm{S} 1$ is available from:

https://doi.org/10.17026/dans-zey-8aec 


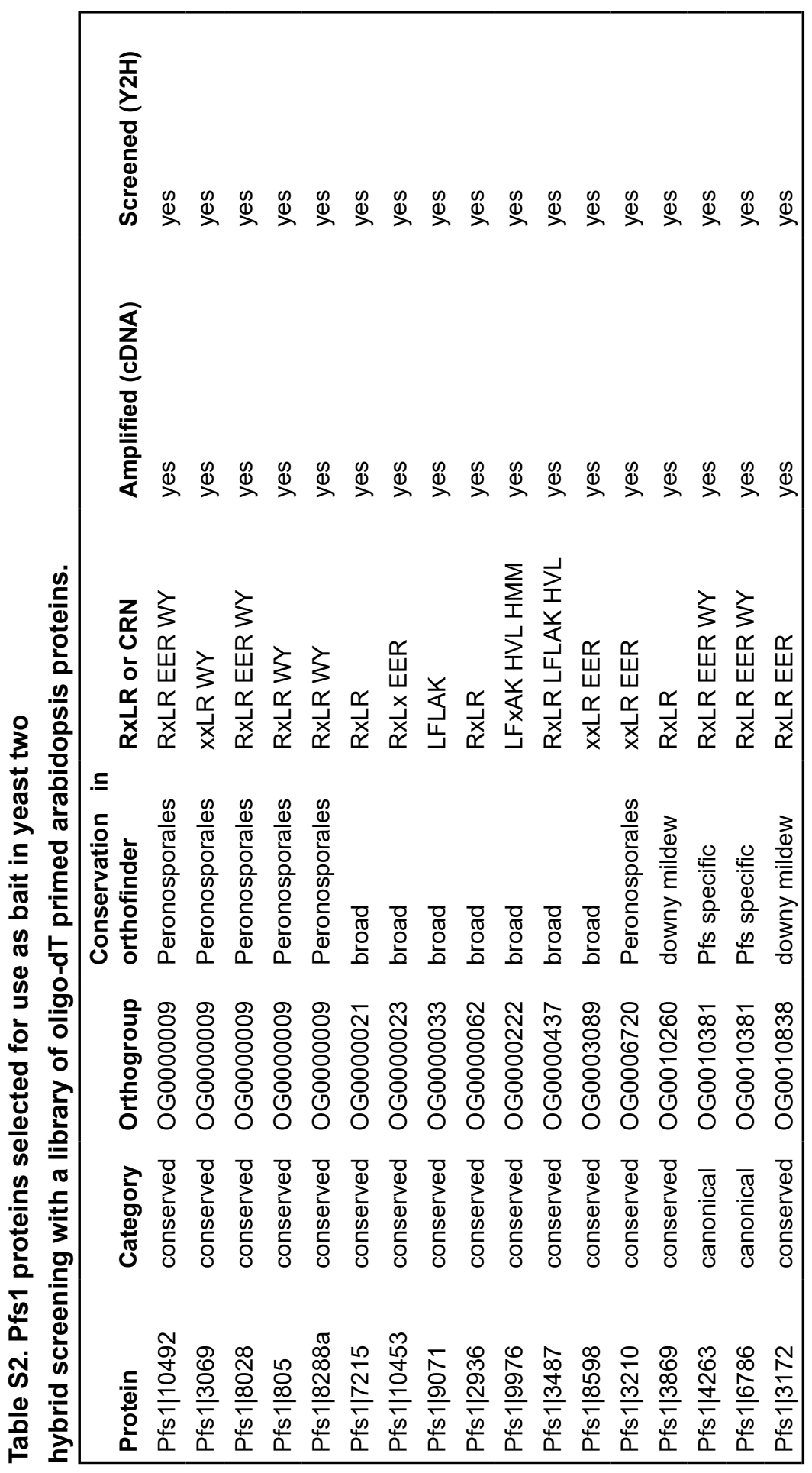




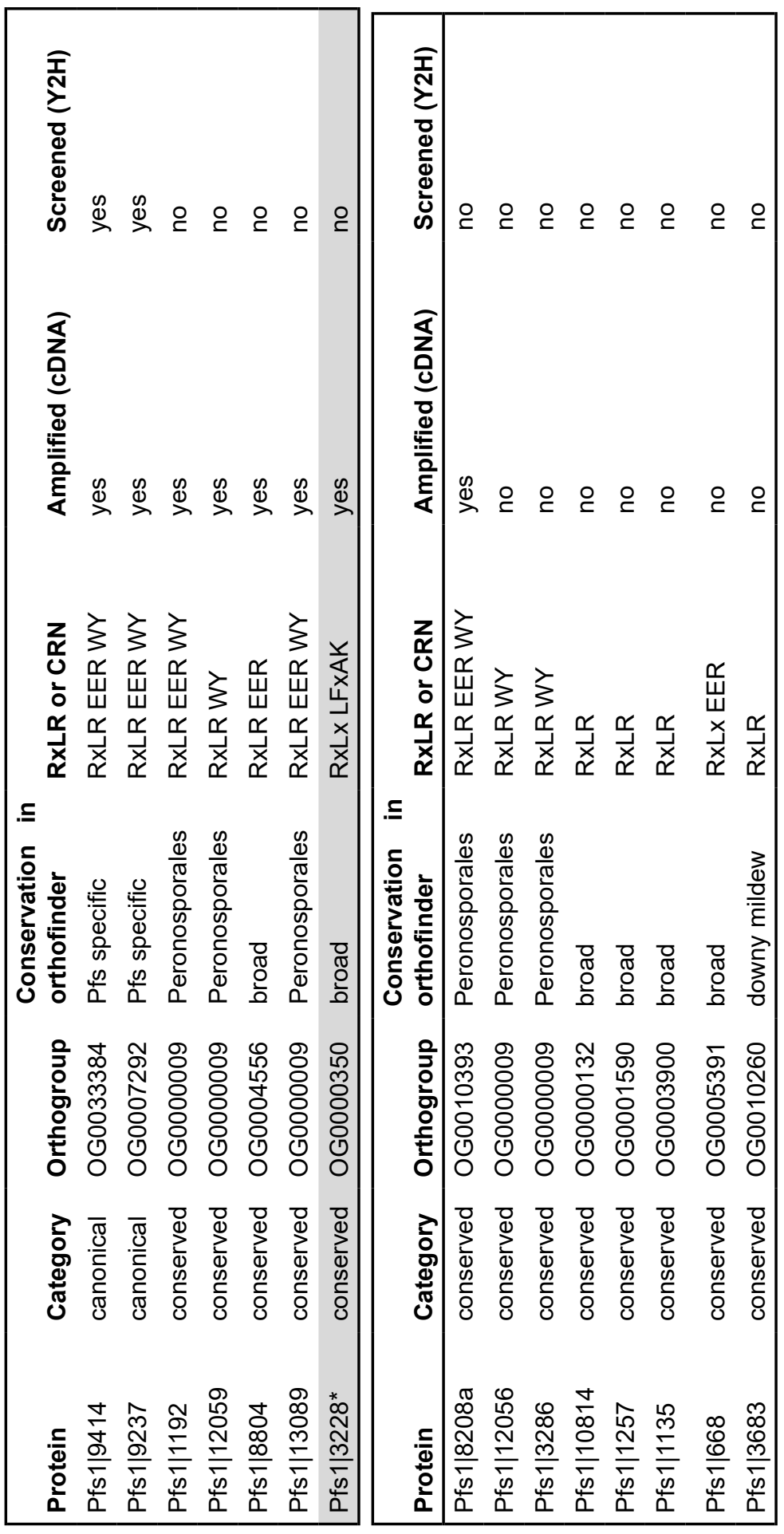




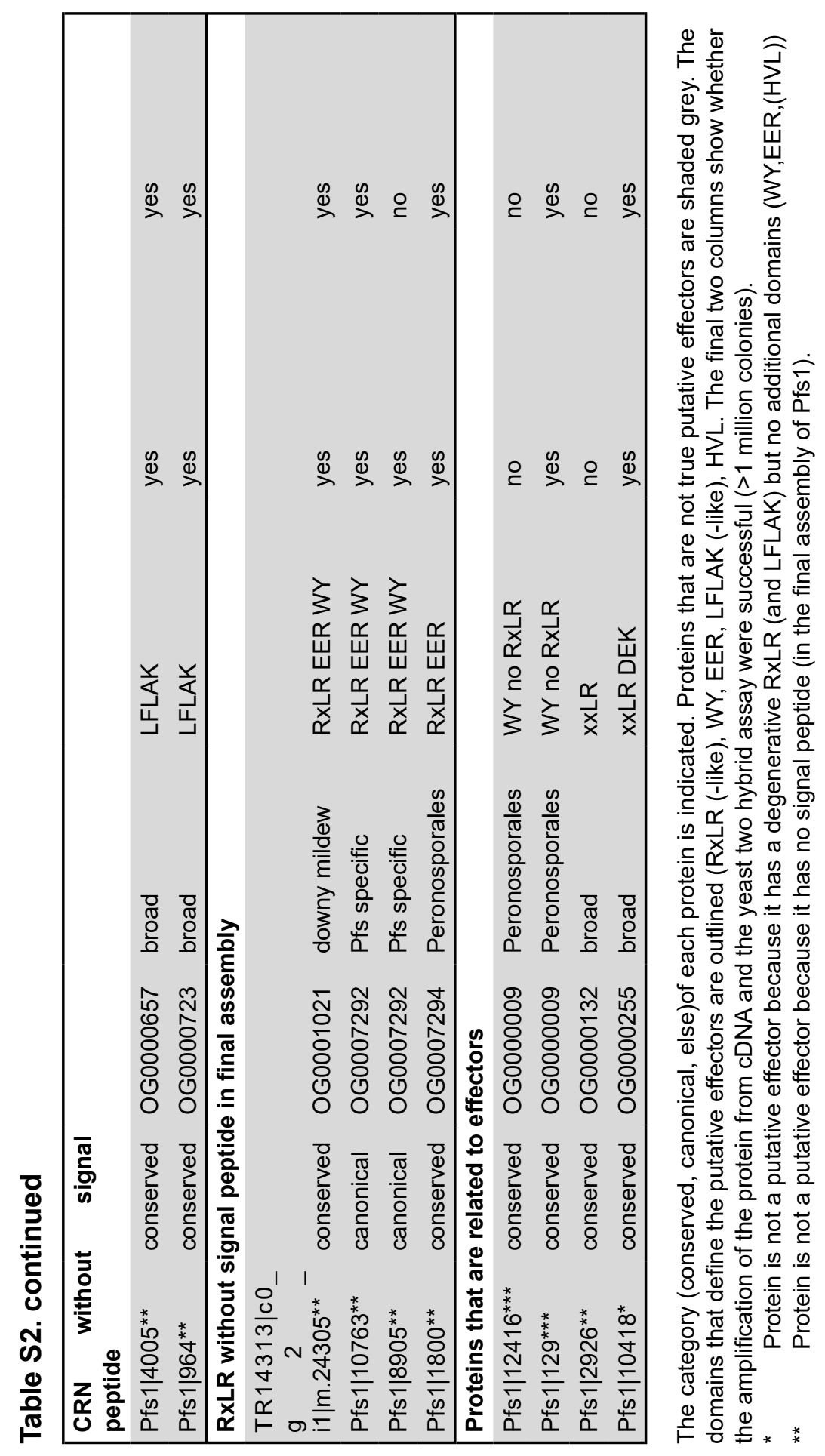


Table S3. Primers

\begin{tabular}{|c|c|}
\hline Gene name & Forward primer \\
\hline pfs1|10492 & GGGGACAAGTTTGTACAAAAAAGCAGGCTCAATGAGTCCAAGTACTATGAAG \\
\hline pfs1|1192 & GGGGACAAGTTTGTACAAAAAAGCAGGCTCAATGACCCCCAAGTC \\
\hline pfs1|3286 & GGGGACAAGTTTGTACAAAAAAGCAGGCTCAATGGTGGAAACTCATAAGTC \\
\hline pfs $1 \mid 3487$ & GGGGACAAGTTTGTACAAAAAAGCAGGCTCAATGCTCTCGGTGCG \\
\hline pfs $1 \mid 8028$ & GGGGACAAGTTTGTACAAAAAAGCAGGCTCAATGAGTCCAAGCACTGT \\
\hline pfs1|8288a & GGGGACAAGTTTGTACAAAAAAGCAGGCTCAATGAATTCCGCGCC \\
\hline pfs1|8804 & GGGGACAAGTTTGTACAAAAAAGCAGGCTCAATGTTGTTTGTGCCTATG \\
\hline pfs1|10453 & GGGGACAAGTTTGTACAAAAAAGCAGGCTTCATGACCAATTTGGAACGACAAACCC \\
\hline pfs $1 \mid 805$ & GGGGACAAGTTTGTACAAAAAAGCAGGCTTCATGAGTCCAAGCACTGTCGAGC \\
\hline pfs1|2936 & GGGGACAAGTTTGTACAAAAAAGCAGGCTTCATGCAACACCTAGTGGCTCCAC \\
\hline pfs1|10418 & GGGGACAAGTTTGTACAAAAAAGCAGGCTTCATGCACCAAGTTGTGCGGAATCC \\
\hline pfs1|8598 & GGGGACAAGTTTGTACAAAAAAGCAGGCTTCATGCGAAATCAATTACGGGGTGCG \\
\hline pfs1|3683 & GGGGACAAGTTTGTACAAAAAAGCAGGCTTCATGGATGTTCCGCTTTCCCTACAG \\
\hline pfs1|12056 & GGGGACAAGTTTGTACAAAAAAGCAGGCTTCATGGCACAATCGAGCAGTGTCAT \\
\hline pfs1|3210 & GGGGACAAGTTTGTACAAAAAAGCAGGCTTCATGGCCAATCTTCGTTCGGAAAAGA \\
\hline pfs $1 \mid 668$ & GGGGACAAGTTTGTACAAAAAAGCAGGCTTCATGGGTAAAGACTATTACGAGATCTTGGG \\
\hline pfs $1 \mid 3228$ & CACCATGGTAACGTTAATATGTGCGA \\
\hline pfs1|9071 & CACCATGATGCTAAAGCTTTTTTGCGTCG \\
\hline pfs1|964 & CACCATGATGGCAAAGCTGTTGTGCGT \\
\hline pfs1|4005 & CACCATGATGTACATGATGTCACTAGCTC \\
\hline pfs1|9976 & GGGGACAAGTTTGTACAAAAAAGCAGGCTTAATGGCCTGCACGATTAAGAAAG \\
\hline pfs1|13089 & CACCATGGGCGACACTCCCATCCCT \\
\hline TR14313* & CACCATGACAAGATCACCTGAGGATGC \\
\hline pfs $1 \mid 1800$ & CACCATGGCTGCCCCGGCAGCTCA \\
\hline pfs1|8905 & CACCATGGTAATGATATGGAGTCCACAG \\
\hline pfs $1 \mid 4263$ & CACCATGGGTGCACATACAGAGCCGT \\
\hline
\end{tabular}




\begin{tabular}{|c|c|}
\hline Gene name & Forward primer \\
\hline pfs1|9237 & CACCATGGTTGTGCTAATATGGAGTCCA \\
\hline pfs $1 \mid 10763$ & GGGGACAAGTTTGTACAAAAAAGCAGGCTTAATGGTTGTGCTAATATGGAGTCCA \\
\hline pfs $1 \mid 6786$ & GGGGACAAGTTTGTACAAAAAAGCAGGCTTAATGCATGTCACAGATGACCAATATG \\
\hline pfs $1 \mid 3172$ & GGGGACAAGTTTGTACAAAAAAGCAGGCTTAATGTGCGGCTGCTTCATTCGTG \\
\hline pfs1|9414 & CACCATGGATCTGTCACCAGAGGATGC \\
\hline pfs $1 \mid 1135$ & GGGGACAAGTTTGTACAAAAAAGCAGGCTTCATGTCGACCAACAGCACACCTCT \\
\hline pfs $1 \mid 1257$ & GGGGACAAGTTTGTACAAAAAAGCAGGCTTCATGTTTGTGAATTTACCTCTTGCTTTCGT \\
\hline pfs $1 \mid 3069$ & GGGGACAAGTTTGTACAAAAAAGCAGGCTTCATGAAGATAACAACTTCCGTCGTTG \\
\hline pfs1|129 & GGGGACAAGTTTGTACAAAAAAGCAGGCTTCATGGAGCCAACACCTTTCGT \\
\hline pfs $1 \mid 12416$ & GGGGACAAGTTTGTACAAAAAAGCAGGCTTCATGACCAAAGTCGAGCCAACAACTT \\
\hline pfs $1 \mid 3869$ & GGGGACAAGTTTGTACAAAAAAGCAGGCTTCATGGATGTCCCGCACTTCGCGCATC \\
\hline pfs $1 \mid 7215$ & GGGGACAAGTTTGTACAAAAAAGCAGGCTTCATGGCCGAAGGGACCGAGCTGACAC \\
\hline pfs $1 \mid 10814$ & GGGGACAAGTTTGTACAAAAAAGCAGGCTTCATGTCGGGTGTTGGGTCTCCG \\
\hline pfs1|2926 & GGGGACAAGTTTGTACAAAAAAGCAGGCTTCATGACTCGCTACAGAGCGAAAGAGG \\
\hline pfs $1 \mid 12059$ & GGGGACAAGTTTGTACAAAAAAGCAGGCTTCATGTTGCTCGCATATTCAGGTTC \\
\hline Gene name & Reverse primer \\
\hline pfs $1 \mid 10492$ & GGGGACCACTTTGTACAAGAAAGCTGGGTCCTATCAATTTTTCATCGGCGGCT \\
\hline pfs $1 \mid 1192$ & GGGGACCACTTTGTACAAGAAAGCTGGGTCCTACTAACCATGAACATCGAAATTGCT \\
\hline pfs $1 \mid 3286$ & GGGGACCACTTTGTACAAGAAAGCTGGGTCCTATTAAAACATCTTTATCAGTGCATCAGC \\
\hline pfs $1 \mid 3487$ & GGGGACCACTTTGTACAAGAAAGCTGGGTCCTATCACTGGCCGTATTTCGTCTTG \\
\hline pfs $1 \mid 8028$ & GGGGACCACTTTGTACAAGAAAGCTGGGTCCTACTAATTTTCCGTCGGTGACTCG \\
\hline pfs $1 \mid 8288 a$ & GGGGACCACTTTGTACAAGAAAGCTGGGTCCTATAAGCCACTTTCGTATCAGCT \\
\hline pfs $1 \mid 8804$ & GGGGACCACTTTGTACAAGAAAGCTGGGTCCTATTACCCACTTGTGTCGTCAA \\
\hline pfs $1 \mid 10453$ & GGGGACCACTTTGTACAAGAAAGCTGGGTCCTATTACAAAAGACACTCAAACCACTGC \\
\hline pfs $1 \mid 805$ & GGGGACCACTTTGTACAAGAAAGCTGGGTCCTATCACAATCTTCTTTTATCCACGAGT \\
\hline pfs1|2936 & GGGGACCACTTTGTACAAGAAAGCTGGGTCCTATTAAGCGAAGTCTGAGCGATCG \\
\hline
\end{tabular}




\begin{tabular}{|c|c|}
\hline \multicolumn{2}{|c|}{ Table S3. continued } \\
\hline Gene name & Reverse primer \\
\hline pfs1|10418 & GGGGACCACTTTGTACAAGAAAGCTGGGTCCTATCAGTTACTCACAATATGGTGGTG \\
\hline pfs1|8598 & GGGGACCACTTTGTACAAGAAAGCTGGGTCCTATCATTCCTCGCTAAACTCTGTCC \\
\hline pfs1|3683 & GGGGACCACTTTGTACAAGAAAGCTGGGTCCTATTACAATATACTTAGGTTGATGAGGCC \\
\hline pfs1|12056 & GGGGACCACTTTGTACAAGAAAGCTGGGTCCTATTACTTTTCCGAGTAAGGTACTACA \\
\hline pfs $1 \mid 3210$ & GGGGACCACTTTGTACAAGAAAGCTGGGTCCTACTACATACTCCCATTACTCGGCG \\
\hline pfs $1 \mid 668$ & GGGGACCACTTTGTACAAGAAAGCTGGGTCCTATTACTTCATCGAAAATAACTTGCGA \\
\hline pfs1|3228 & TCAATCGACCGCCGGAGTC \\
\hline pfs1|9071 & TTATGACGTTAAAAATGTCTCAAG \\
\hline pfs $1 \mid 964$ & TCATCTTGAAGCTTGCCTTCC \\
\hline pfs1|4005 & TTATAACAGTGTCAGACCACTG \\
\hline pfs1|9976 & TCATTTCACAATTGAGTCATCG \\
\hline pfs1|13089 & TCACGCGTATTGAGCCTTAAT \\
\hline TR14313* & CTACTTGTCGCCCAGAAACG \\
\hline pfs $1 \mid 1800$ & CTATGGCGATTCGTCATGGT \\
\hline pfs1|8905 & CTACTGACCATCGTCTTTTCG \\
\hline pfs $1 \mid 4263$ & TCTAAGGTATTAAATCTTTCCCA \\
\hline pfs1|9237 & CTACTGACCATCGTCTTCTC \\
\hline pfs1|10763 & GGGGACCACTTTGTACAAGAAAGCTGGGTCCTACTGACCATCGTCGATAC \\
\hline pfs $1 \mid 6786$ & GGGGACCACTTTGTACAAGAAAGCTGGGTCCTACTCCGGAAGCGACTTTA \\
\hline pfs1|3172 & GGGGACCACTTTGTACAAGAAAGCTGGGTCCTAAACGGTAGTCGTCGATC \\
\hline pfs1|9414 & TCACGAGCTTAGGTCGAGTT \\
\hline pfs1|1135 & GGGGACCACTTTGTACAAGAAAGCTGGGTCCTACTATTTCTCATGTCTTTCGTTGTCTGA \\
\hline pfs1|1257 & GGGGACCACTTTGTACAAGAAAGCTGGGTCCTATTAGGCAAGAGCTGTACGTTGG \\
\hline pfs1|3069 & GGGGACCACTTTGTACAAGAAAGCTGGGTCTTACTCTGCTTCCCGCATTC \\
\hline pfs1|129 & GGGGACCACTTTGTACAAGAAAGCTGGGTCTCAACTCGATTCCTTGTTGCC \\
\hline pfs1|12416 & GGGGACCACTTTGTACAAGAAAGCTGGGTCCTAATCTTCCTCCСTCTTTGTCTT \\
\hline Gene name & Reverse primer \\
\hline pfs1|3869 & GGGGACCACTTTGTACAAGAAAGCTGGGTCTCACAGATTCACAATAGCATCCAA \\
\hline pfs1|7215 & GGGGACCACTTTGTACAAGAAAGCTGGGTCTTACGAGCACTTCTCCGATTT \\
\hline pfs $1 \mid 10814$ & GGGGACCACTTTGTACAAGAAAGCTGGGTCTCAATTTATAGCGGTCCG \\
\hline pfs1|2926 & GGGGACCACTTTGTACAAGAAAGCTGGGTCTCATTCACGGCCCTCATCAT \\
\hline pfs1|12059 & GGGGACCACTTTGTACAAGAAAGCTGGGTCCTACCTGTCTTGTGGTACAAATCCA \\
\hline
\end{tabular}

Primers used for amplification of effectors for Gateway BP and entry cloning. 


\section{Supplemental information 1}

Upon manual curation an additional start codon was identified (bold) for protein Pfs1|1800-00002 and Pfs1|4091. The newly predicted sequences have a signal peptides (italics). The putative translocation domain is underlined.

$>$ Pfs $1 \mid 1800$

MRLLCVLVMAVAALTASNSAAPAAQATDDIKLWQANPQDRIDTMDALHIKTRPKRMLRGVSNEDYDVDNEVDNDSSTSDTFLEDRIQQKLAKKSVRLQLYEVWYDCRFTVEKVSKHLTISSSNKLFPTYHALKKGYERYCARPHDKPIRPVSDETCKESYLAQILDNHDESP

$>$ Pfs $1 \mid 4091$

MRLDSILFVAVLFANLSISSTRSMSTVVKSVTFNQEDVPAHGLGVKTTNEERTLGSPVVEKAAEEITSNVPESLEGVLNLQNEMEKLRLGEDKSIMDNPHFNNWVRDLAKTIDTSSAAKEVITKVSELYGDEGLLKMLNILIKDEVGEVEGVLQGELKSALMASWEKQEMSARGVFEQLKLDQETDHTLYIQL LTMWVRYTKENNLETKTVITSYDGNISLMLFEGLSEIEGTEEIVKSLQDSLMTFWAGRELSAEDLFSTLKLDNGGSKSNYDMWVKYVAQEFDTLQPTKEVILKSSEVFGEEGLLKMLNALDLKEVGQDIQSELTSALMASWKEKEKKPVEVLMLLESELMPEFNHKVNIGRIKMWNQYVNENFEYPGLVELLAMGQIKNIAHKTIILEDLKQINLAAKDARKNSENAE

Although the Pfs1|10763 has a 33 amino acid hydrophobic N-terminus (italics), the signal prediction for this protein is just below the threshold. The effector bait for this protein lacks the hydrophobic part. The putative translocation domain is underlined. 
$>\mathrm{PfS} 1 \mid 10763$

MACTIKKVKHLFWYMVLVTVVVLIWSPQTMVKMVVASQHAPRDARENRRLRPSSYMKEGVNGGDEERRLNIDLNLPALPDNLFKVLKIDPEVGEKIEATIPEVGEKLEAKVPEVDENFEGWIPEVDEKIEATIPEVDENVKSLLKNTRLHEWANF PKDTTVEEYINAAIVKLTELVTKNGKETVASMLKAAKNDPATKQIAANLERILVLSHELNEDAFKMLSVGQADLRAFLASPLLKNYLELHLQLTGDFSLGPLVTMLLEHYSDAALAKKLLEVHSKDAAAEMAEEVSTYHTDHWENTYSGEILFERLGLKKAGIHMVDSPVWDTWTMYMAMRKGINKFAEPDLAEVAIPVLRKNFHDKVLLDWFNAAKTDAGVTRAGFLKTALDEARTAEGKAPLTISKRKGIDNELLVPTKRQRIDDGQ 

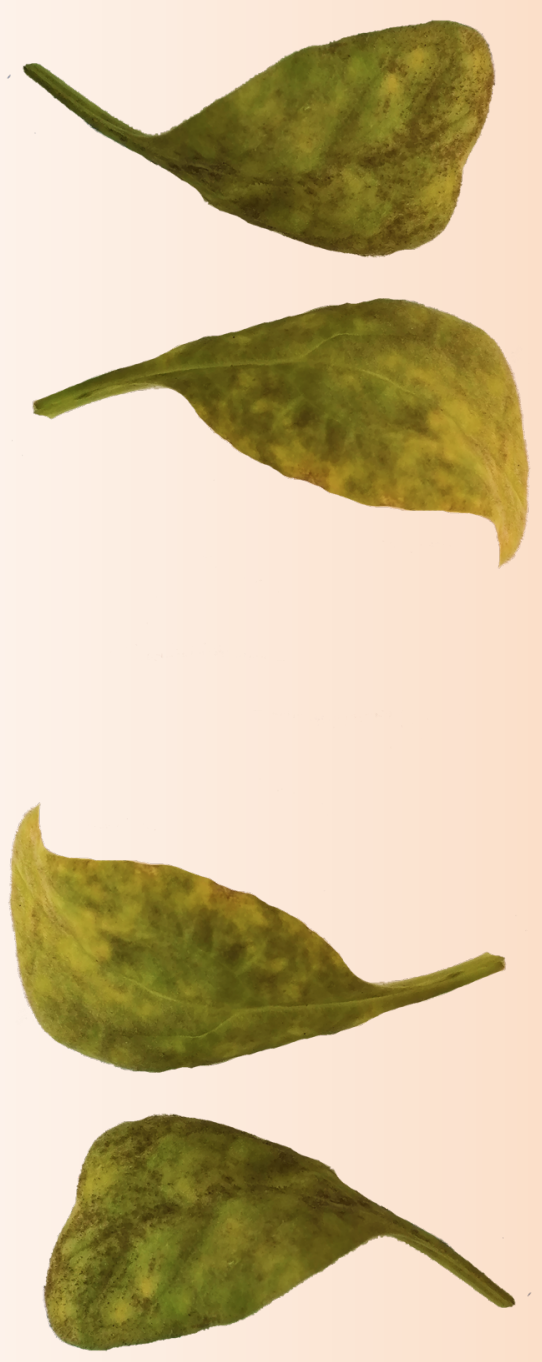


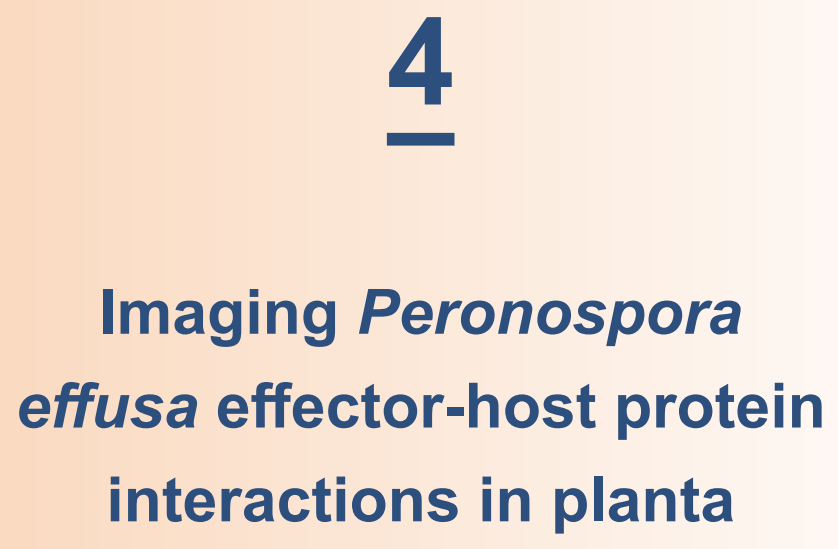

Manon Neilen ${ }^{1}$, Claudia Meisrimler ${ }^{1,2}$,

Guido Van den Ackerveken ${ }^{1}$

${ }^{1}$ Plant-Microbe Interactions, Department of Biology, Utrecht University, Padualaan 8, 3584 CH Utrecht, the Netherlands

${ }^{2}$ Current address: School of Biology, University of Canterbury, Private bag 4800, Christchurch 8140 , New Zealand. 


\section{Abstract}

Phytopathogenic oomycetes secrete effector proteins into plant tissue to establish and maintain their infection. These effectors can either act in the apoplast or translocate into host cells and function there. Hosttranslocated effectors, like RxLR and Crinkler (CRN) effectors, are known to interact with plant components like RNA and proteins to manipulate cell processes. The genome of Peronospora effusa (Pfs), the obligate biotrophic oomycete that causes downy mildew on spinach, encodes over 100 candidate RxLR and CRN effectors. Previously, interacting Arabidopsis proteins of 18 effectors were identified by yeast twohybrid screening. Eight candidate effectors that interacted with fourteen Arabidopsis protein fragments were selected for further functional validation. The subcellular localization of the effectors and their interactors was visualized by expression of fluorescent fusion proteins in Nicotiana benthamiana leaves. All but two of the 14 tested Arabidopsis interactors and their spinach orthologs localized with the candidate effector in the same subcellular compartment. The subcellular localization of six plant interactors and two effector proteins was altered upon co-expression of the effector and interactor proteins. Furthermore, seven plant proteins showed a more stable signal when co-expressed with the effector. 


\section{Introduction}

Peronospora effusa (Pfs) is an obligate biotrophic oomycete that causes downy mildew on spinach and is a major threat to spinach production worldwide [30, 32]. Its hyphae can penetrate the leaf tissue and grow between plant cells, which they invaginate to form haustoria, the sites of interaction between the oomycete and plant cells [37]. The pathogen secretes proteins and other molecules to establish and maintain a successful infection [237]. These secreted proteins are called effectors and have evolved to establish compatibility between the pathogen and the spinach host. Effectors can either function between (apoplast) or within plant cells (host-translocated effectors) [40]. Host-translocated effectors are known to interact with plant proteins to suppress immunity and alter cellular processes for the benefit of the pathogen [238]. Two types of host-translocated effectors are known in oomycetes, the RxLR and Crinkler (CRN) effectors [56, 65].

It was shown that RxLR and CRN effectors of downy mildew species localize to many different subcellular compartments in plant cells. Hyaloperonospora arabidopsidis RxLR effectors were found to mainly target two cellular compartments; the nucleus (66\% of tested effectors) and membranes [209]. Similar targeting of host-translocated effectors was found in the grapevine downy mildew Plasmopara viticola and sunflower downy mildew Plasmopara halstedii [239]. Remarkably, P. viticola also has three effectors that localize to chloroplasts and mitochondria [240], while a number of $P$. halstedii effectors were found to target chloroplasts, the tonoplast, endoplasmic reticulum (ER) and the Golgi system. Several CRN effectors were found to localize to the plant nucleus upon their expression in planta [56] The next challenge is to elucidate why individual host-translocated effectors show such a specific localization in plant cells and how that contributes to pathogen virulence.

Previously we identified $99 R \times L R$ and $14 C R N$ candidate genes in the genome of the $P$. effusa reference race 1 (Pfs1) (Chapter 1 and 2 ). In a first step towards understanding the function of effectors in plant cells, Arabidopsis protein fragments that interact with 18 candidate effectors 
were identified in a yeast two-hybrid ( $\mathrm{Y} 2 \mathrm{H})$ screen (Chapter 3). Here we describe the further analysis of eight candidate effectors and their 14 interacting Arabidopsis proteins. The spinach orthologs of the Arabidopsis proteins were identified and the subcellular distribution of all plant and effector proteins was assessed using transient in planta expression of fluorescent protein fusions. Co-expression of host interactors and effectors showed that subcellular localization of 12 Arabidopsis and all 14 spinach proteins overlapped with that of the effector, supporting their presumed interaction in planta. Furthermore, upon co-expression we observed the re-localization of two effector proteins and six interacting plant proteins. Another 7 plant proteins were stabilized upon co-expression with the effector. These results strongly suggest that the identified protein-protein interactions are indeed occurring in planta.

\section{Results}

\section{Identification of spinach orthologs of Arabidopsis interactors}

Orthologs of the Arabidopsis interactors were identified in the published

4 proteome of spinach variety Sp75 [241] using a reciprocal BlastP search (TAIR10 Arabidopsis proteome). For ten Arabidopsis proteins we identified spinach proteins that gave a reciprocal best hit, suggesting they are orthologs (Table 1). For four other Arabidopsis interactors, low sulphur upregulated 1 (LSU1, At3g49580), serine-arginine rich protein (SR-rich, At2g19390), glycin-rich protein (glycin-rich, At5g56100), HOMOLOG OF BEE2 INTERACTING WITH IBH 1 (HBI1, At2g18300), the highestscoring spinach proteins were more closely related to other Arabidopsis proteins in the reciprocal blast, suggesting a multiple-to-one orthology between the Arabidopsis and spinach proteins. The relations between the Arabidopsis and spinach proteins that were not reciprocally matched were clarified using protein trees generated with all significantly related predicted proteins of both species as identified by a BlastP search (Fig. S1). 
In the case of HBI1 we found a 2-to-1 orthology between HBI1, At4g34530 and spinach protein (Spo) Spo06524. Even though Spo06524 is the spinach protein with the highest similarity to $\mathrm{HBI} 1$, it is more similar to the Arabidopsis HBI1 paralog At4g34530. In addition, a 2-to-1 orthology was found between At2g19390, At4g29790 and Spo24468 and a 6-to-1 orthology was observed for At5g56100 and five Arabidopsis paralogs with a single spinach protein, Spo12707. However, the similarity significance score of At5g56100 and Spo12707 (BlastP E-value; 1.48e-03) is above $1 \mathrm{e}^{-05}$ and their potential orthology should thus be considered with caution (Fig. S1). For LSU1 the protein tree did not improve our understanding of orthology to spinach because of the unresolved branches. The best BlastP hit, Spo22402, was therefore included as the ortholog. Both Arabidopsis Prenylated Rab acceptor 1 interactors, PRA1.F2 and PRA1. F3, had the same spinach protein, Spo20420, as a reciprocal best hit. In total,13 spinach proteins, referred to as "protein orthologs", were added to the 14 Arabidopsis interactors for further analysis. The protein alignments between the Arabidopsis and spinach orthologs are shown in Fig. S2. 
Table 1. Spinach protein orthologs or best BlastP hits of Arabidopsis effector-interactors.

\begin{tabular}{|c|c|c|c|c|c|c|}
\hline $\begin{array}{l}\text { Arabidopsis } \\
\text { effector- } \\
\text { interactor }\end{array}$ & At-code & $\begin{array}{l}\text { Best } \\
\text { BlastP hit } \\
\text { Spinach }\end{array}$ & E-value & $\begin{array}{l}\text { Reciprocal B } \\
\text { BlastP hit } \\
\text { Arabidopsis }\end{array}$ & E-value & $\begin{array}{l}\text { Reciprocal } \\
\text { best hit }\end{array}$ \\
\hline NAC014 & At1g33060 & Spo22390 & $3.85 \mathrm{e}^{-88}$ & At1g33060 & $7.51 \mathrm{e}^{-88}$ & Yes \\
\hline PRA1.F2 & At1g55190 & Spo20420 & $1.50 e^{-60}$ & At1g55190 & $1.06 e^{-61}$ & Yes \\
\hline USPA & At3g11930 & Spo22595 & $8.65 e^{-65}$ & At3g11930 & $5.69 e^{-66}$ & Yes \\
\hline PRA1.F3 & At3g13720 & Spo20420 & $3.10 e^{-62}$ & At3g13720 & $1.10 e^{-5} 5$ & Yes \\
\hline AT3G13800 & At3g13800 & Spo20405 & 0 & At3g13800 & 0 & Yes \\
\hline AHAS & At3t48560 & Spo20077 & 0 & At3g48560 & 0 & Yes \\
\hline NAGK & At3g57560 & Spo15075 & $5.17 e^{-152}$ & At3g57560 & $1.03 e^{-151}$ & Yes \\
\hline FBN1a & At4g04020 & Spo20112 & $2.96 \mathrm{e}^{-127}$ & At4g04020 & $4.39 \mathrm{e}^{-127}$ & Yes \\
\hline NAC078 & At5g04410 & Spo16524 & $3.86 e^{-128}$ & At5g04410 & $1.85 e^{-123}$ & Yes \\
\hline RAF2 & At5g51110 & Spo14154 & $4.10 e^{-65}$ & At5g51110 & $7.89 e^{-69}$ & Yes \\
\hline SR-rich & At2g19390 & Spo24468 & $7.11 e^{-121}$ & At5g22450 & $4.59 e^{-167}$ & No \\
\hline LSU1 & At3g49580 & Spo22402 & $3.22 e^{-10}$ & At5g24655 & $1.33 e^{-18}$ & No \\
\hline Glycin rich & At5g56100 & Spo12707 & $1.48 e^{-03}$ & At4g25140 & $4.09 e^{-52}$ & No \\
\hline $\mathrm{HBI} 1$ & At2g18300 & Spo06524 & $1.75 e^{-52}$ & At4g34530 & $8.32 e^{-64}$ & No \\
\hline
\end{tabular}

Subcellular localization of candidate Pfs host-translocated effectors and their interactors in planta

The coding sequence of the candidate host-translocated effectors (without signal peptide), Arabidopsis interactors and spinach orthologs were cloned into plant expression vectors for making fusions to fluorescent proteins (FPs). Effectors were fused to yellow fluorescent protein (YFP) and the interacting plant proteins to cyan fluorescent protein (CFP) at their N-terminal unless otherwise indicated. The fusion proteins were produced in Nicotiana benthamiana by transient Agrobacterium-mediated expression to examine the subcellular localization of the proteins. Originally, we attempted to produce the fusion proteins in spinach leaves, but found that the level of expression was much lower compared to $N$. benthamiana, and in most cases insufficient for microscopic analysis of 
subcellular localization (Fig. S3). Hence, the gene fusion proteins were visualized in $N$. benthamiana leaves.

Six candidate host-translocated effectors were found to localize in punctate structures (PS) (Fig. 1). Two of the six PS-localized effector fusions, Pfs1|1800 and Pfs1|3869, were also detected in the nucleus and cytoplasm, and one (Pfs1|3172) also in the endoplasmic reticulum (ER). PS in plant cells could represent structures like Golgi associated vesicles, lipid bodies [242], autophagosomes [243] or vacuoles [244]. The PS to which the candidate host-translocated effectors localize are very similar in size and shape to Golgi vesicles, as demonstrated by their resemblance to the Golgi marker, soybean $\alpha-1,2-$ mannosidase I [245, 246] (Table 2). However, we cannot be entirely sure as PS are also observed in mitochondria and chloroplasts as demonstrated by the localization of the yeast cytochrome oxidase subunit IV (ScCOX41-29GFP) that serves as a mitochondrial marker in N. benthamiana [247].

Of the eight tested effector fusion proteins, two localized elsewhere. Pfs1|10763 was located solely and strongly in large nuclear speckles, while Pfs1|9071 was visible in the nucleus and cytoplasm (Fig. 1). The experimentally-determined localization of the fusion proteins was compared to the predicted effector localization as assigned by the machine learning algorithm LOCALIZER, that has a specific configuration for effector proteins and by the deep learning algorithm Deeploc. Surprisingly, the predicted and experimental localization matched only for Pfs1|3172 that localizes to Golgi-like PS (Table 2). 

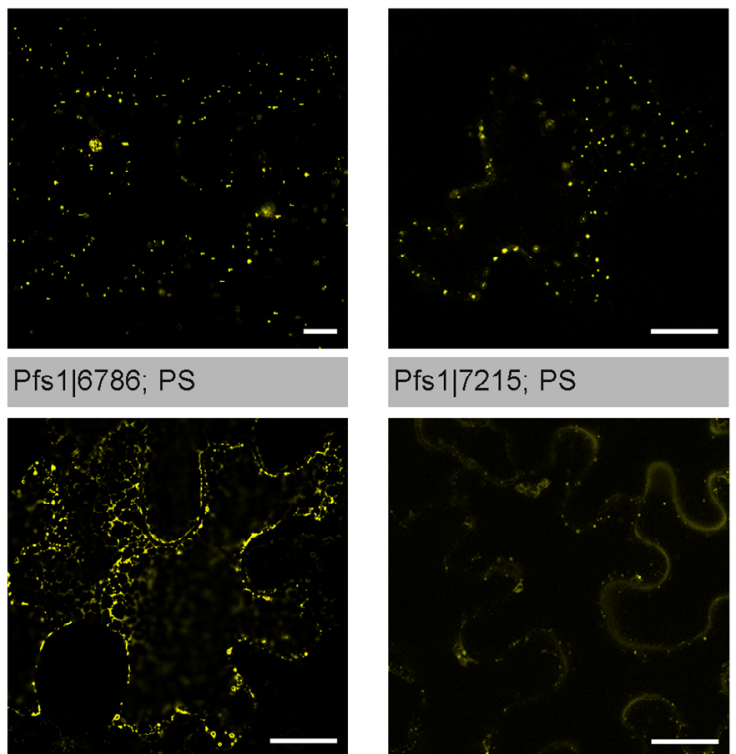

Pfs $1 \mid 3172 ; E R$
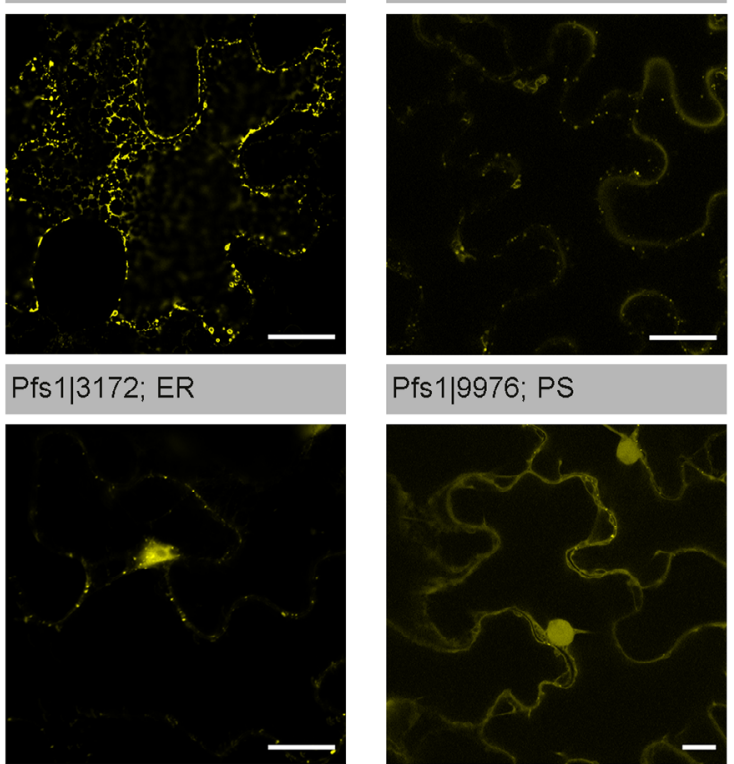

Pfs1|9976; PS
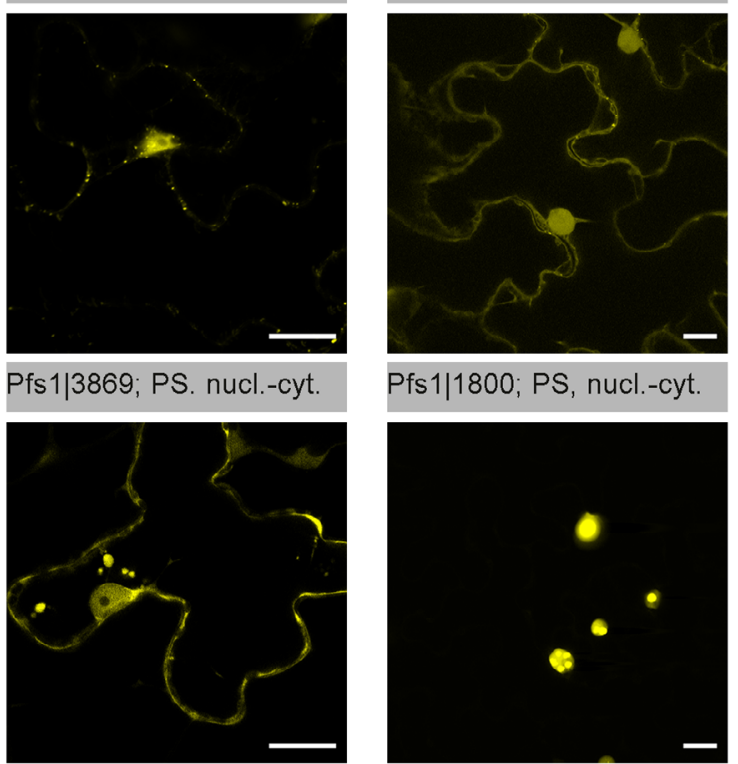

Pfs1|1800; PS, nucl.-cyt.

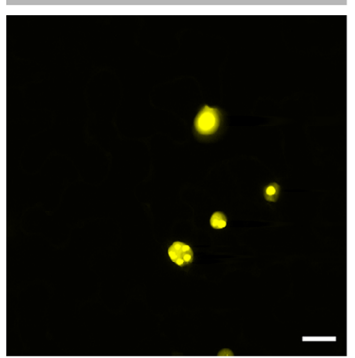

Pfs $1 \mid 9071$; nucl.-cytoplasm

Pfs1|10763; nucl. speckles

Figure 1. Subcellular localization of eight candidate Pfs1 effectors in $\mathbf{N}$. benthamiana.

Pfs1 effectors fused to YFP (N-terminal) were produced in $N$. benthamiana leaves by Agrobacterium-mediated transient expression. Protein localization is indicated in the figure. Images were taken $\sim 48$ hours after infiltration. Images are representative for $n \geq 2$ observations, except for Pfs1|6786 (5 cells) and Pfs $1 \mid 3869$ (3 cells) that were only imaged once. Scale bars $=20 \mu \mathrm{m}$. 
Table 2. Experimental and predicted subcellular localization of $\mathbf{N}$-terminal YFP fluorescent protein fusions of individually expressed candidate effector proteins of Pfs 1 in N. benthamiana

\begin{tabular}{llll}
\hline Effector & Subcellular localization & LOCALIZER & Deeploc \\
\hline Pfs1|9071 & nucleo-cytoplasmic & - & mitochondrium (soluble) \\
Pfs $1 \mid 9976$ & punctate structures (PS) & nucleus & cytoplasm (soluble) \\
Pfs $1 \mid 1800$ & PS, nucleo-cytoplasmic & - & plastid (membrane) \\
Pfs $1 \mid 10763$ & large nuclear speckles & nucleus & extracellular (soluble) \\
Pfs $1 \mid 6786$ & PS & - & mitochondrium \\
& & & (membrane) \\
Pfs $1 \mid 3172$ & ER & - & Golgi (membrane) \\
Pfs $1 \mid 3869$ & PS, nucleo-cytoplasmic & - & extracellular (soluble) \\
Pfs $1 \mid 7215$ & PS & - & extracellular (soluble) \\
\hline
\end{tabular}

LOCALIZER predicts the localization of effector proteins to chloroplast, mitochondria (transit peptide) and nucleus (nuclear localization signal (NLS)). For effectors, it does not consider the first 20 amino acids. Note that effector protein sequences with the signal peptide sequences removed were used for Deeploc predictions.

Similarly, the Arabidopsis interactors and their spinach orthologs were each individually expressed in $N$. benthamiana in the absence of the corresponding effector protein. The observed localization was compared to subcellular localization data retrieved from SUBA4 (Table 3) [248]. The SUBA consensus (SUBAcon) prediction is the localization retrieved from the combined predicted and experimentally observed (if available) subcellular localizations. The data from the SUBA4 experimental database are separately shown. For six of the fifteen Arabidopsis proteins the observed and reported localization overlapped, but for eight interactors a different localization was observed. For NAC078, (NO APICAL MERISTEM (NAM), ARABIDOPSIS THALIANA TRANSCRIPTION ACTIVATION FACTOR (ATAF1/2) and CUP-SHAPED COTYLEDON (CUC2) 078, At5g04410) we were not able to determine the localization, as the fusion protein could not be detected.

Nine out of fourteen Arabidopsis interactors were found to localize in PS in the imaged $N$. benthamiana cells (Fig. S4, Table 3). All PSlocalized interactors were also found in other subcellular compartments, like chloroplasts (1x), the ER (4x), the ER, nucleus and cytoplasm (1x), the 
ER and nucleus (1x) the ER and cytoplasm (1x), and nucleus (1x). Two interactors, NAC014 (At1g33060) and HBI1 (At2g18300) were observed in nuclear speckles. Nuclear speckles are visible as dot-like structures within the nucleoplasm [249]. Both interactors were hard to observe as their protein levels were low if present at all, suggesting that the fusion proteins may be unstable, or their cellular levels strongly regulated. Universal stress protein A (USPA) (At3g11930) was found to be soluble and was observed in the cytoplasm and nucleus. Acetohydroxyacid synthase (AHAS, At3g48560) was observed in plastids.

Ten of the fourteen tested spinach fusion proteins localized at least partially to the same cell compartments as their Arabidopsis orthologs. Three could not be compared because the spinach (3x) or Arabidopsis (1x) proteins could not be visualized due to low intensity of the fluorescent signal upon expression of the fusion proteins. For a single Arabidopsisspinach pair, the protein localizations were remarkably different: the Arabidopsis AHAS fusion protein was observed in chloroplasts and PS while its ortholog Spo20077 localized to the PS only. 


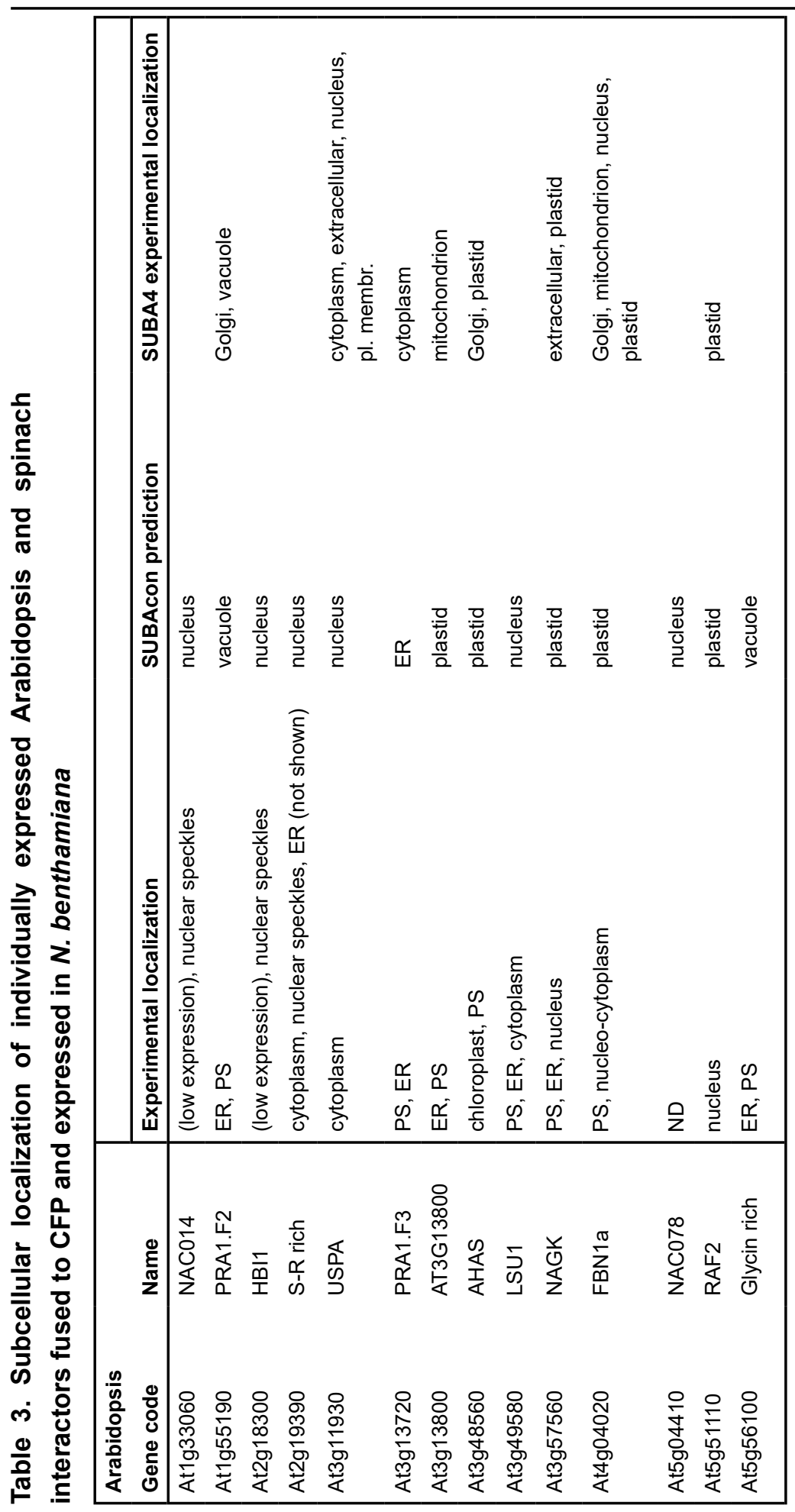




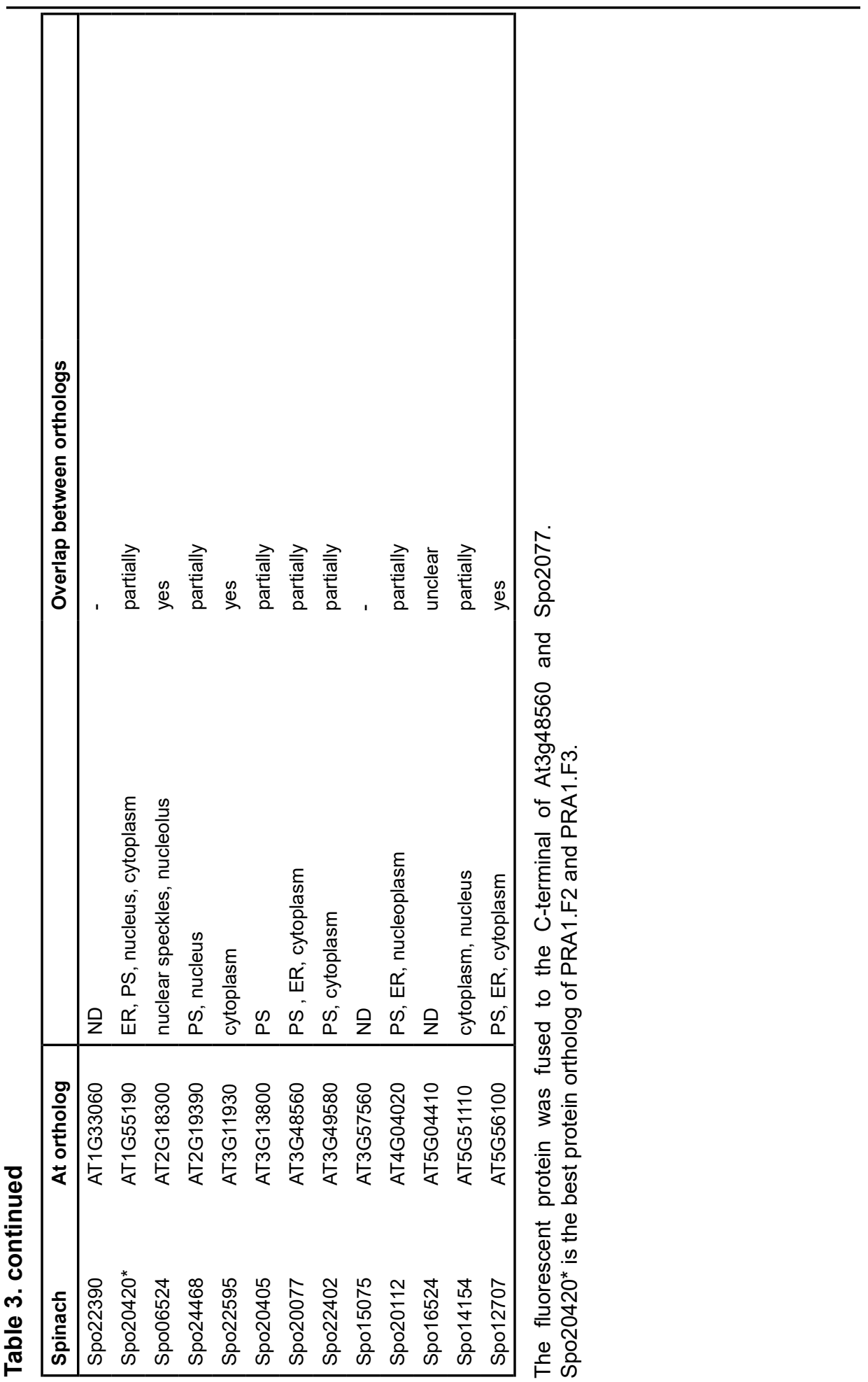




\section{Co-localization of candidate effectors and their interactors}

The results above describe the subcellular localization of the individual candidate effectors and their interactors. It is important to determine the subcellular localization of effector and corresponding interactor proteins when they are co-expressed in the same cells. When such co-localization experiments demonstrate the presence of both interaction partners in the same subcellular compartment, it supports their proposed interaction. Also, co-expression may alter the localization or protein stability of one or either interaction partners. We observed that all but two of the Arabidopsis and all spinach interactor fusions tested localized, at least partially, to the same subcellular compartment as the corresponding candidate host-translocated effector (Table 4). The two exceptions, PRA1. F2 (At1g55190) and PRA1.F3 (At3g13720) localize to the ER and PS, whereas the candidate effector Pfs $1 \mid 9071$ is observed in the nucleus. The observation that these proteins do not co-localize, and their localization is not altered by co-expression suggests that PRA1.F2/F3 and Pfs1|9071 do not interact in planta and could thus be considered false positive $\mathrm{Y} 2 \mathrm{H}$ interactors. Interestingly, the localization of the ortholog of PRA1.F2 and PRA1.F3 in spinach, Spo20420, was in the ER like PRA1.F2/F3, but also in the cytoplasm. Therefore, the spinach ortholog co-localized with effector Pfs1|9071 in the cytoplasm. The presence of intact Spo20420 fused to CFP was confirmed by Western blot detection, indicating that the cytoplasmic signal is not derived from free CFP resulting from fusion protein break-down (Table S1). 


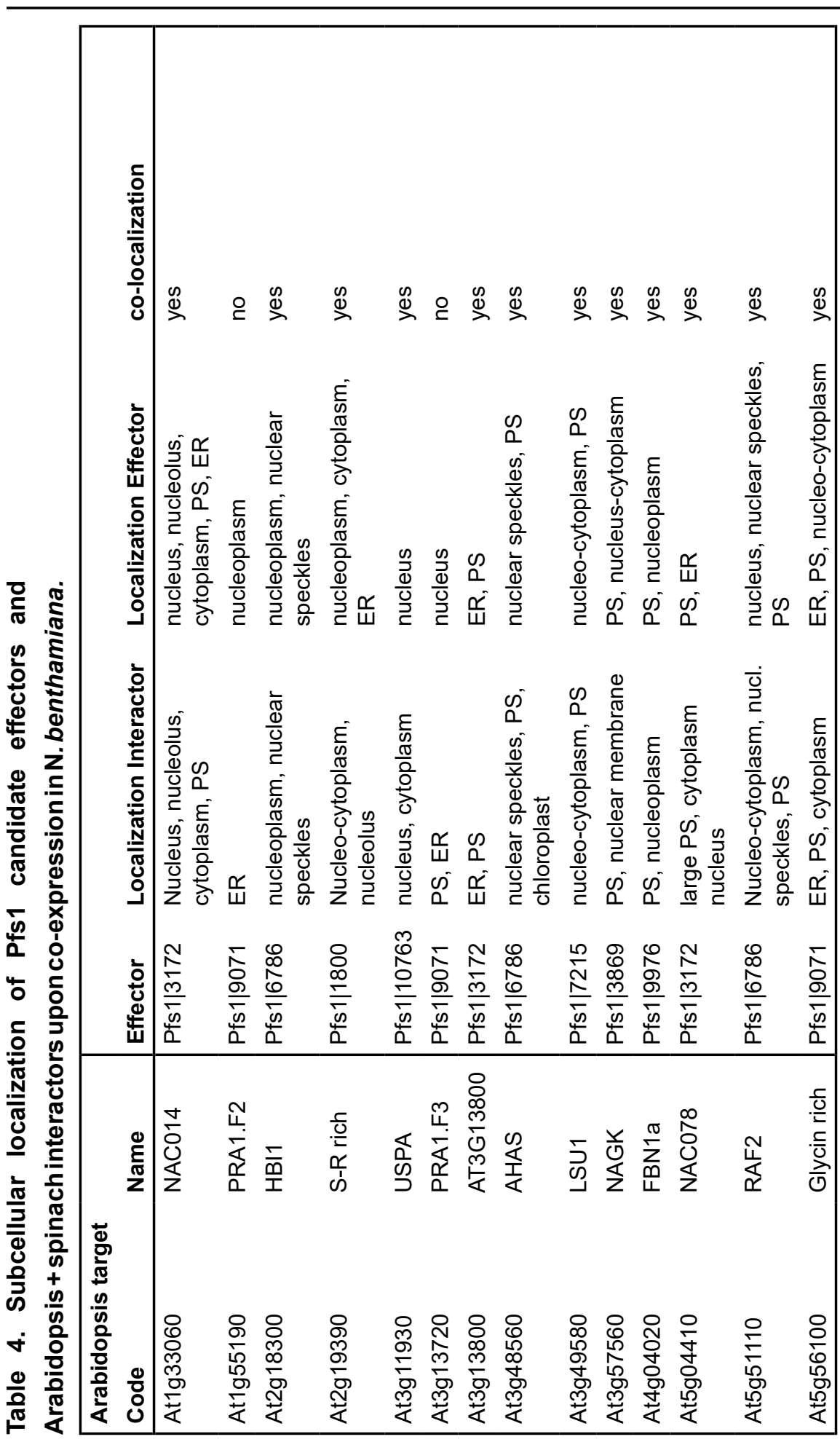




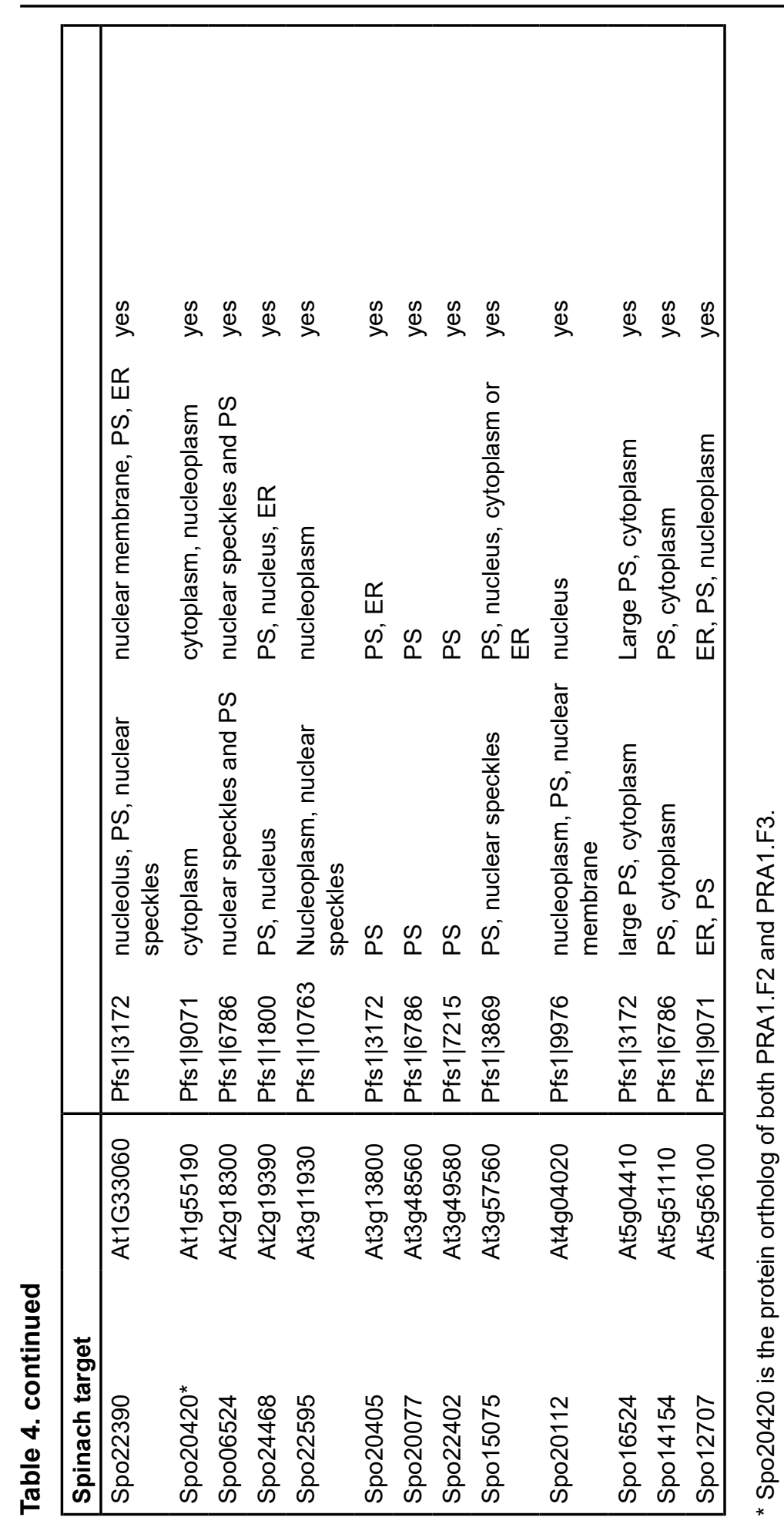




\section{Stabilization of interacting proteins upon effector co-expression}

Three Arabidopsis (NAC014, NAC078 and HBI1) and three spinach fusion proteins (NAC04, NAC078 and NAGK-ortholog Spo15075) were barely visible by confocal microscopy when individually expressed in $N$. benthamiana. The fluorescent signal was generally low, and if fluorescence was observed, it was often concentrated in large (as opposed to the small PS) cytoplasmic dots. The origin of the large dots is unclear but they resemble the subcellular localization of proteasome core particle subunit PAG1 [250] which could suggest that the proteins are targeted for degradation by the proteasome.

Surprisingly, all of these unstable interactors could be successfully imaged when co-expressed with their cognate candidate host-translocated effector. The fluorescent signals of the effector co-infiltrated plant fusion proteins were more abundant within the transformed cells compared to the signals of the individual interactors.

Protein stabilization after co-expression with the candidate host-translocated effector Pfs1|3869 was observed for spinach protein Spo15075. When co-expressed the Spo15075 fusion protein was clearly visible in PS, nucleoplasm and nuclear speckles, while individually it was only observed in large fluorescent dots (Fig. 2). The presence of the candidate effector appears to stabilize the interactor signal. Colocalization of the interactor and candidate effector occurred in the PS and the nucleoplasm but not in the nuclear speckles. For the Arabidopsis ortholog ACETYLGLUTAMATE KINASE (NAGK, AT3G57560) there was no difference if it was expressed individually or together with the candidate effector; in both cases it localized to the PS, the nucleoplasm and possibly the ER. 


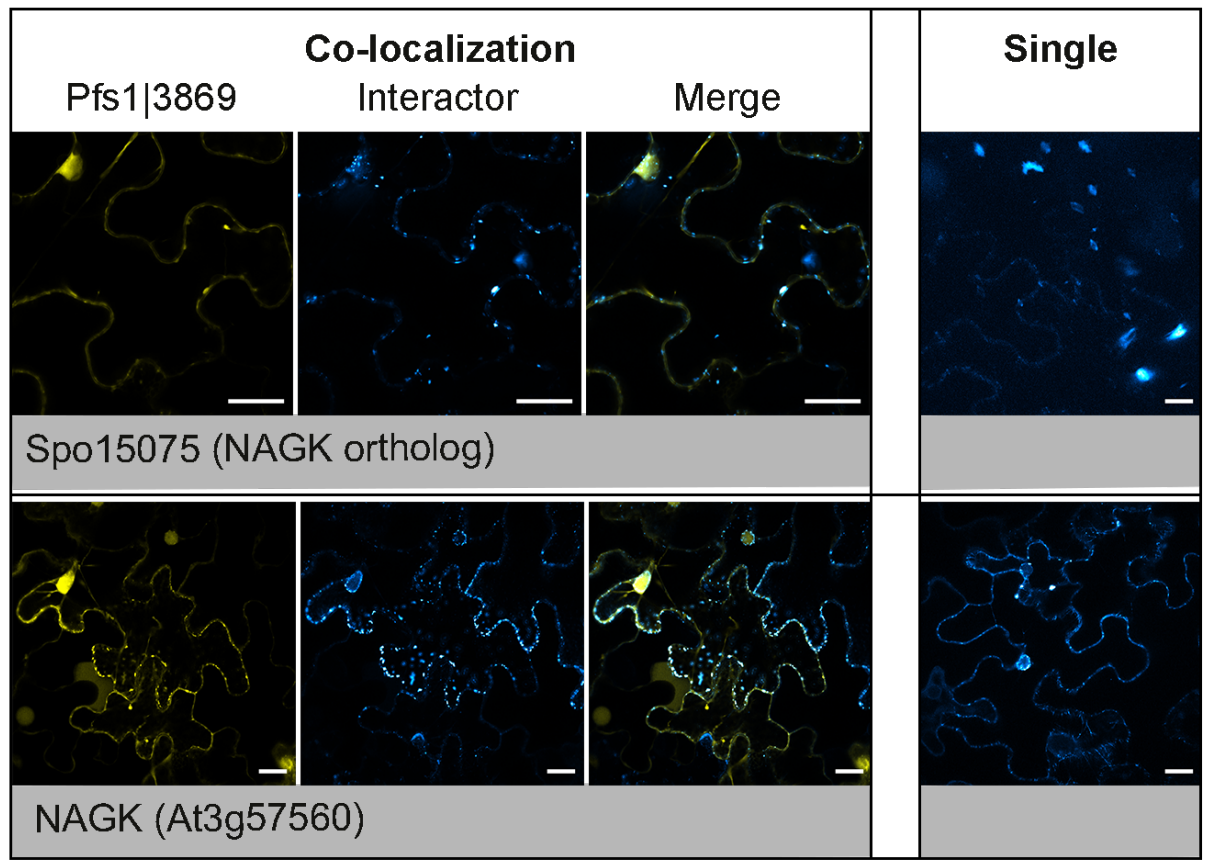

Figure 2. Co-localization of Arabidopsis NAGK (At3g57560) and its protein ortholog Spo15075 with candidate effector Pfs1|3869 in N. benthamiana. Plant interactors fused to CFP and candidate effectors fused to YFP (N-terminus) were transiently expressed in N. benthamiana. The Spo15075 fusion protein was observed in large cytoplasmic speckles when expressed independent of effector Pfs 1|3869 but was clearly visible in PS, nucleoplasm and nuclear speckles upon co-expression. Subcellular localization and intensity of the Arabidopsis NAGK fusion protein was not altered upon co-expression with the candidate effector. Images were taken $\sim 72$ hours after infiltration and are representative of $>8$ cells. Scale bars $=20 \mu \mathrm{m}$.

Another example of stabilization of interactor proteins is for the NAC transcription factors NAC014 and NAC078. Despite numerous attempts, no clear localization was found for NAC014 and NAC078 nor for the corresponding orthologs Spo22390 and Spo14154, respectively, due to low intensity of the signal. In our studies, NAC014 and Spo14154 (NAC078 ortholog) could be weakly detected in nuclear speckles in one sample (Fig. 3) but this was not reproduced. When co-expressed with Pfs1|3172, the interactor fusions could be visualized (Fig. 3). NAC014 was observed in the nucleus (nuclear membrane and nucleolus), 
cytoplasm, ER and PS. Co-localization with Pfs1|3172 was observed in all compartments. The spinach NAC014 ortholog, Spo22390, was primarily observed in PS that clearly overlay the ER, and in the cytoplasm. Colocalization with the effector was observed in the cytoplasm. NAC078 localized in the nucleus and cytoplasm, PS and in large cytoplasmic speckles. Pfs1|3172 co-localized with NAC078 in the large cytoplasmic speckles, nucleus and cytoplasm. The effector was clearly localized in the ER together with a weak NAC078 signal. The localization of the NAC078 ortholog, Spo16524, resembled that of NAC078 but with a more pronounced ER localization.

In a study in lettuce (Lactuca sativa), it was previously shown that NAC transcription factors interact with host-translocated effectors of the downy mildew pathogen Bremia lactucae. It was found that a truncated fusion protein of LsNAC069, lacking the NAC domain, was more stable compared to the full length protein [169]. Therefore, a truncated version of Arabidopsis NAC014 expressed as a CFP-tagged fusion protein was expressed in N. benthamiana. All NAC protein fragments that interacted with Pfs $1 \mid 3172$ in the $\mathrm{Y} 2 \mathrm{H}$ assays lacked the NAC domain and showed a strong interaction with the effector so the NAC domain is not required 4 for that (Chapter 3). The truncated protein fusion was, indeed, more stable and showed a strong signal in the ER, cytoplasm and PS when individually expressed (Fig. 4). Also, the presence of the truncated but not the full-length protein could be confirmed by western blot analysis using an antibody against the FP (Table S1). When co-expressed with effector $\mathrm{Pfs} \mid 3172$ co-localization was observed in the ER, PS and potentially the nucleus. 


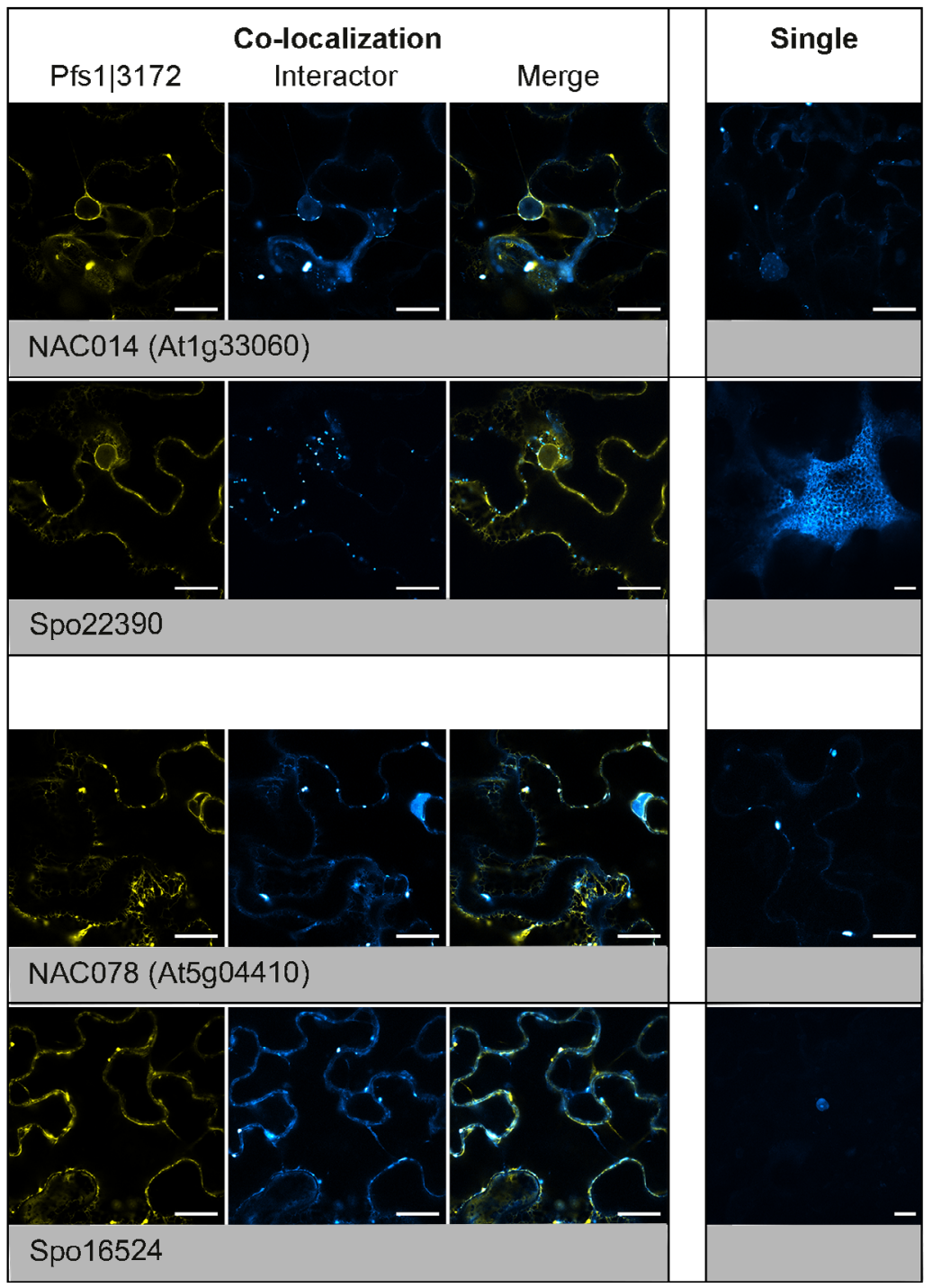

Figure 3. Stabilization of NAC transcription factor fusions by co-expression with candidate effector Pfs1|3172 in $N$. benthamiana.

Plant interactors fused to CFP and candidate effectors fused to YFP (N-terminus) were transiently expressed in $N$. benthamiana. The subcellular localization of both Arabidopsis and spinach NAC transcription factors was observed at higher intensity when co-expressed with candidate effector Pfs $1 \mid 3172$. Images were taken $\sim 48$ hours after infiltration. Images are representative of $>8$. Scale bars $=20 \mu \mathrm{m}$. 


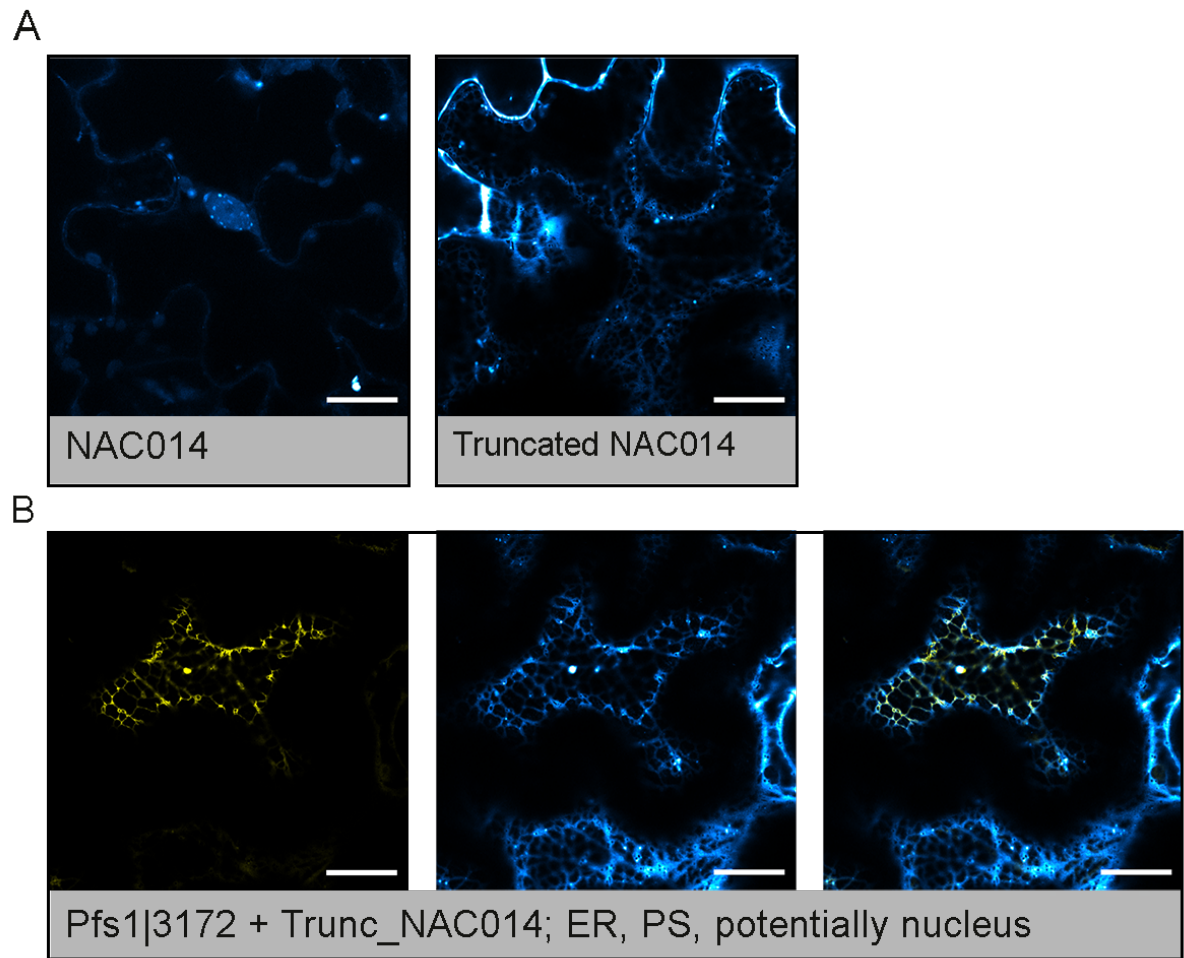

Figure 4. Subcellular localization of individually and co-expressed full

4 length and truncated NAC014 (At1g033060) proteins in N. benthamiana.

Plant interactors fused to CFP and candidate effectors fused to YFP (N-terminus) were transiently expressed in N. benthamiana. (A) The signal of the truncated protein fusion is stronger compared to the full length one. (B) Co-localization of the truncated NAC014 and Pfs1 candidate effector Pfs $1 \mid 3172$ is observed in the ER, PS, and potentially in the nucleus. Images were taken $\sim 48$ hours after infiltration and are representative of 3 cells (NAC014, 2 technical replicates) 10 cells (truncated NAC014), 4 cells (co-expression). Scale bars $=20 \mu \mathrm{m}$. 


\section{Co-expression of effector and interactor candidates results in re-localization of proteins}

RxLR effector candidate Pfs $1 \mid 6786$ was found to interact with multiple Arabidopsis proteins in the $\mathrm{Y} 2 \mathrm{H}$ screen (Chapter 3). Interestingly, the co-expression studies showed that the presence of the effector alters subcellular localization of the Arabidopsis AHAS and spinach Spo6524 (HBI1 ortholog) proteins. Moreover, the subcellular localization of the candidate effector itself changed upon co-expression with the interactors. The effector fusion protein predominantly localized in PS when expressed individually and shifted to a predominantly nuclear localization when co-expressed with AHAS (At3g48560), HBI1, Spo06524, and Rubisco Assembly Factor 2 (RAF2, At5g51110) (Fig. 5). Within the nucleus, the candidate effector was observed in the nucleoplasm, and in nuclear speckles, but remained visible in PS as well (Fig. 5).

Pfs1|6786 interactors AHAS and Spo6524 (HBI1 ortholog), changed localization upon co-expression as well. The signal of AHAS shifted from plastids and PS when expressed individually to nuclear speckles, PS and plastids when co-expressed with Pfs $1 \mid 6786$. The spinach ortholog (Spo20077) did not clearly localize to the plastids nor changed localization when co-expressed with Pfs1|6786. Nevertheless, Spo20077 co-localized with Pfs1|6786 to the PS. Presently, it is not clear whether the observed PS were located in the cytoplasm, plastid, mitochondria or the ER-Golgi system.

In a first experiment, Spo06524 (HBI1 ortholog) localized to plant nuclear speckles and nucleolus when expressed individually and shifted to PS and nuclear speckles when co-expressed with Pfs1|6786. Imaging the Arabidopsis HBI1 fusion protein was particularly difficult due to a low fluorescent signal, resulting in the observation of only four transformed cells in five independent replicates. Co-expression of Pfs1|6786 and $\mathrm{HBI} 1$ seemed to stabilize $\mathrm{HBI} 1$ and alter the subcellular localization of HBI1 ortholog Spo06524. The HBI1 fusion protein was observed in nuclear speckles, both in the individual and co-expression assays. Co- 
expression resulted in relocalization of the predominantly PS-localized Pfs 1 |6786 effector to the nucleus. Co-expression of effector candidate and interactors seemed to stabilize HBI1 and alter the subcellular localization of the $\mathrm{HBI}$ ortholog Spo06524.

The final interactor that altered the subcellular localization of Pfs1|6786, RAF2, showed a strong nuclear signal in individual- and in co-expression experiments. However, cells that showed PS localization of RAF2 and Pfs1|6786 and no nuclear signal from Pfs1|6786 upon coexpression were observed as well. The ortholog Spo14154 predominantly localized to the nucleus when expressed individually, but co-expression with Pfs1|6786 resulted in a PS signal and large fluorescent speckles in the cytoplasm. Both for RAF2 as for Spo14154, the subcellular localization of the fluorescent signal was unstable, and the obtained results did not allow to draw a conclusion about their localization and potential re-localization. Remarkably, both effector Pfs1|6786 and RAF2 were strongly concentrated in the nucleolus and nuclear speckles when co-expressed. The concentration of the fluorescent signals in nuclei in the Spo14154 co-localization and the RAF2/Spo14154 individual localization experiments was too strong to observe the subnuclear localization of the 4 proteins (Fig. 5). 
A

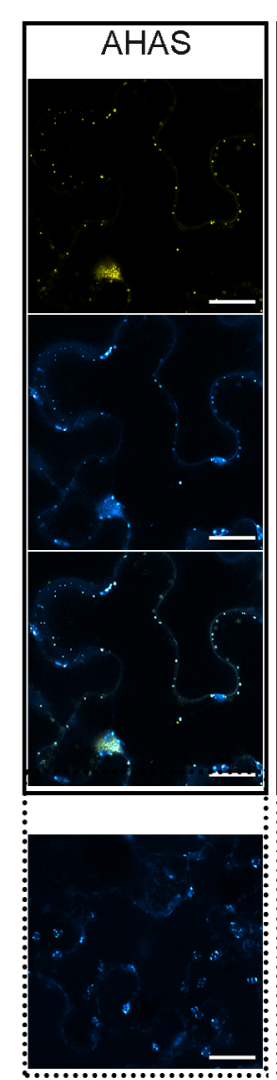

Co-localization

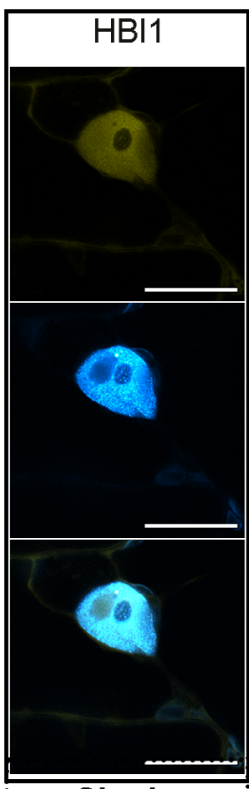

Single

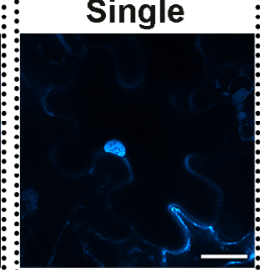

Single

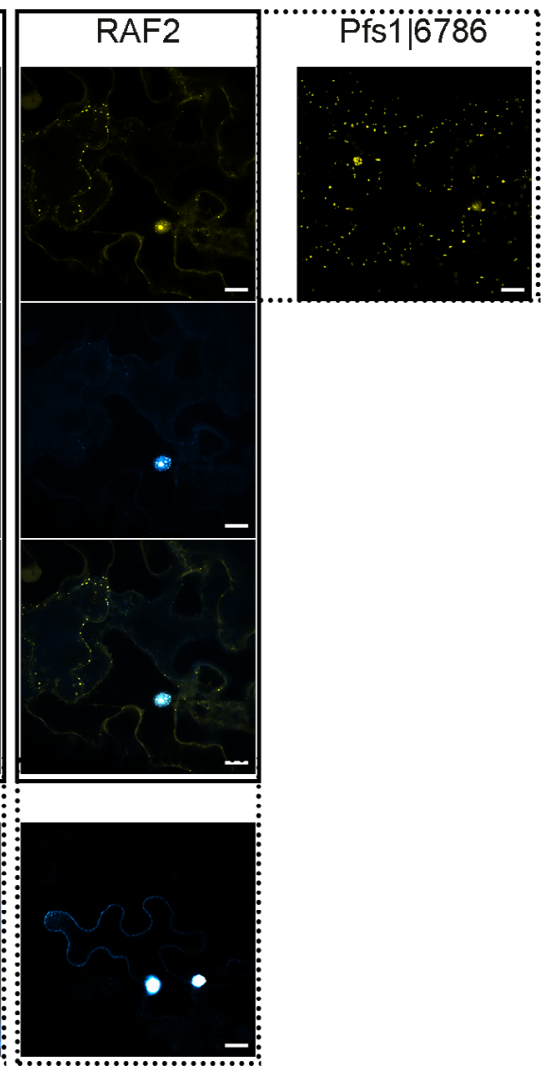

Figure 5. The subcellular localization of candidate effector Pfs $1 \mid 6786$ and four out of six of its candidate interactor proteins changed upon coexpression in $\mathbf{N}$. benthamiana cells.

The subcellular localization of candidate effector Pfs $1 / 6786$ and its three tested Arabidopsis interactors (A) and their spinach orthologs (B) are shown. The subcellular localization of Pfs $1 \mid 6786$ changed from PS to the nucleus (nucleoplasm, nucleolus and nuclear speckles) upon co-expression with AHAS, HBI1 and RAF2 (a, dashed line). The subcellular localization of AHAS (A), Spo06524 (b) and possibly RAF2 (A) and Spo14154 (B) where altered upon co-expression. The expression of HBI was stabilized upon coexpression. The co-expression experiments are representative of 2 cells (HBI1), 7 cells (Spo06524), 8 cells (Spo14154). Images were taken $\sim 48$ hours post infiltration. Scale bars $=20 \mu \mathrm{m}$. 
Co-expression of candidate RxLR effector Pfs1|10763 with its interactors USPA and ortholog Spo22595 induced re-localization and stabilization of the plant proteins (Fig. 6). During individual expression, both plant proteins were visible in the cytoplasm. The signal of Spo22595 could only be observed in a few cells. USPA was occasionally visible in the nucleoplasm. USP and Spo22595 are small $\sim 25$ and $23 \mathrm{kDa}$ proteins. Even when fused to the $27 \mathrm{kDa}$ CFP protein, they might passively move through the nuclear pores into the nucleus [251, 252]. Upon co-expression with Pfs1|10763, both interactors showed a very strong nuclear signal. The Arabidopsis interactor still occurred in the cytoplasm, whereas the spinach ortholog was only visible in the nucleus.

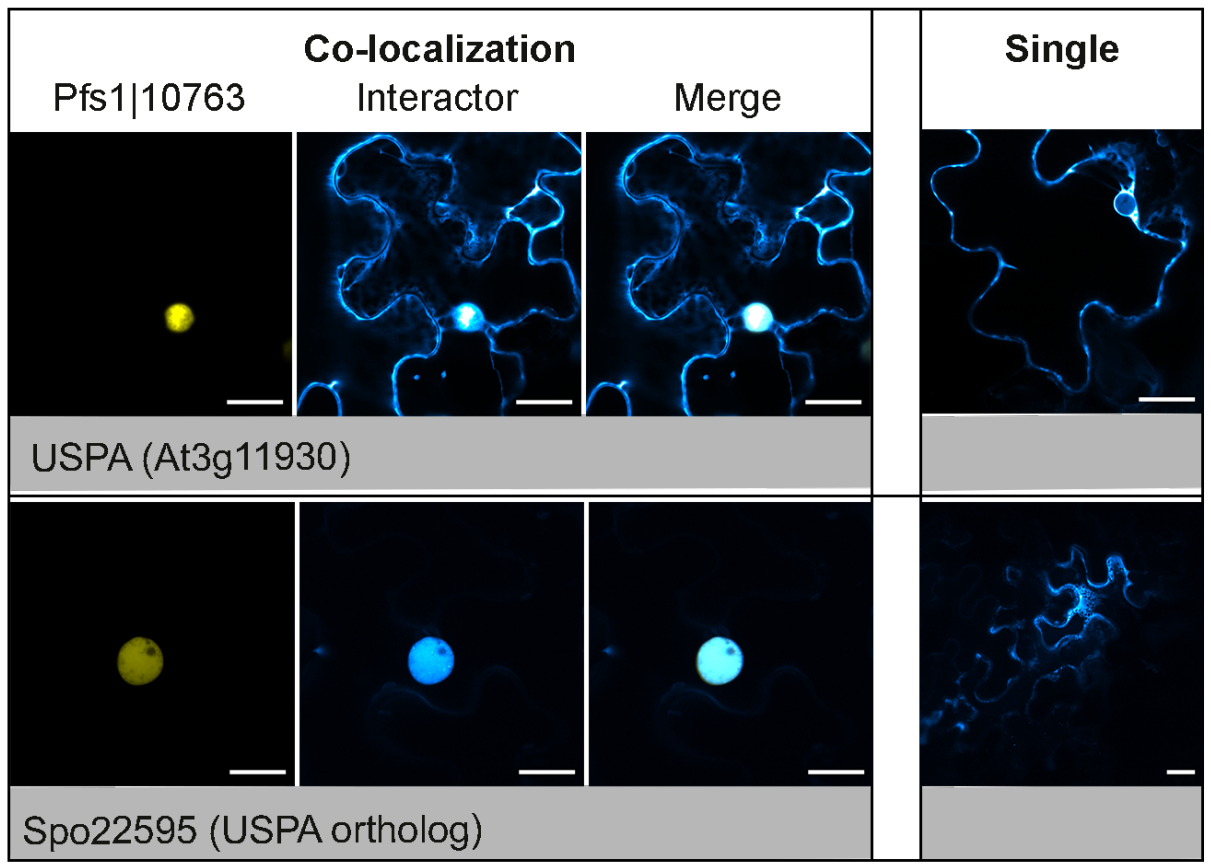

Figure 6. Nuclear co-localization of Arabidopsis USPA (At3g11930) and its protein ortholog Spo22595 with candidate effector Pfs1|10763 in $N$. benthamiana.

Plant interactors fused to CFP and candidate effectors fused to YFP (N-terminus) were transiently expressed in N. benthamiana. The expression of USP and Spo22595, shifted from cytoplasm to nuclear upon co-expression with Pfs1|10763. Images were taken $\sim 48$ hours after infiltration and are representative of $>4$ cells for the individual samples. Scale bars $=20 \mu \mathrm{m}$. 
Re-localization of both the interactor and candidate hosttranslocated effector protein was observed upon co-expression of candidate CRN effector Pfs1|9976 and Fibrillin 1a (FBN1a) (AT4g04020). The FBN1a and Pfs1|9976 fusion proteins re-localized from cytoplasm to the nucleoplasm, whereas localization to PS was maintained (Fig. 7). Co-expression of Pfs1|9976 and FBN1a directed the proteins towards the nucleus. Co-expression of FBN1a ortholog Spo20112 and Pfs1|9976 resulted in relocation of the candidate effector but not the interactor to the nucleus. Spo20112 was observed in PS, ER and nucleus so it colocalized with Pfs1|9976 in the nucleus. 


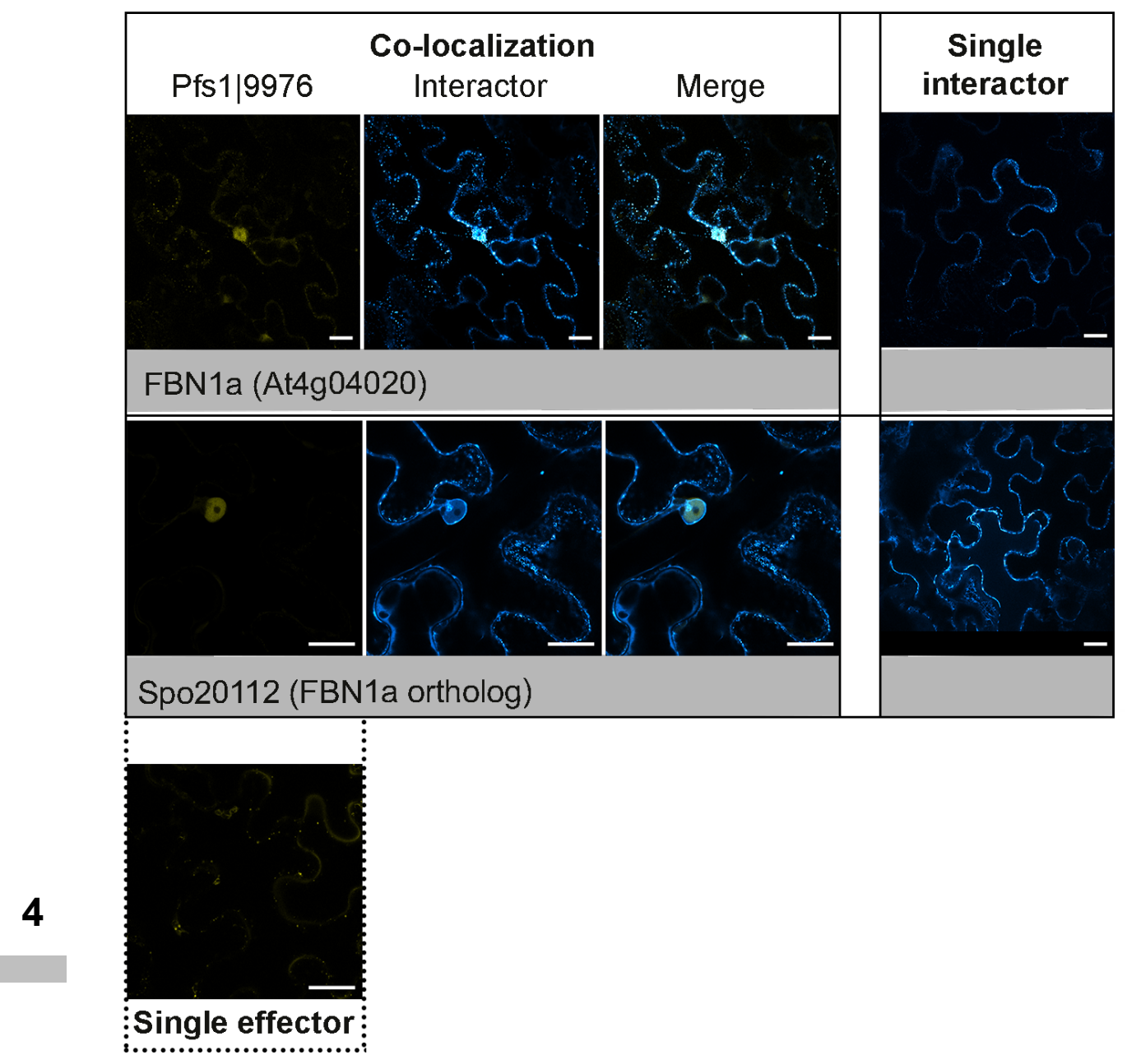

Figure 7. Co-localization of Arabidopsis FBN1a (At4g04020) and its protein ortholog Spo15075 with candidate effector Pfs1|9971 in N. benthamiana.

Plant interactors fused to CFP and candidate effectors fused to YFP (N-terminus) were transiently expressed in $N$. benthamiana. The expression of FBN1a, not Spo15075, shifted from PS and cytoplasm to PS and nucleus upon co-expression with Pfs1|9971. Pfs1|9971 re-localized from PS to the nucleoplasm upon co-expression with both FBN1a and Spo15075. Images were taken $\sim 72$ hours after infiltration and are representative of $>5$ cells. Scale bars $=20 \mu \mathrm{m}$. 
Finally, the SR-rich protein (At2g19390) showed a subnuclear re-localization from the nuclear speckles to the nucleoplasm where it co-localized with the RxLR effector Pfs $1 \mid 1800$ (Fig. 8). The ortholog Spo24468 was also observed in the nucleus when expressed individually.



Figure 8. Co-localization of Arabidopsis SR-rich protein (At2g19390) and its protein ortholog Spo24468 with candidate effector Pfs1|1800 in N. benthamiana.

Plant interactors fused to CFP and candidate effectors fused to YFP (N-terminus) were transiently expressed in N. benthamiana. The expression of At2g19390, not Spo24468, shifted from nuclear speckles and cytoplasm to nucleo-cytoplasm upon co-expression with Pfs 1|1800. Images were taken $\sim 48$ hours after infiltration and are representative of $>5$ cells. Scale bars $=20 \mu \mathrm{m}$.

The interactor proteins that did not stabilize or re-localize upon co-expression with the candidate host-translocated effectors are summarized in Fig. S5. LSU1 and its ortholog Spo22402 co-localized with Pfs1|7215 in the nucleus and cytoplasm and PS. At5g56100 and Spo12707 co-localized in the ER and PS and also in the nucleus and cytoplasm with Pfs1|7215. The co-localization of candidate effector and interactor support their putative interaction in planta. 


\section{Discussion}

Oomycete phytopathogens translocate effectors into plant cells to establish infection $[56,65]$. Inside the plant cells, RxLR and CRN proteins interact with plant proteins and other components to manipulate plant processes, like immune signaling, metabolism and protein transport to the benefit of the oomycete $[41,42,43]$. Recently, it was demonstrated that delivery of RxLR effector Pi04314, an RxLR effector of $P$. infestans was independent of the conventional ER-to Golgi secretion system and that the RxLR motif was required for translocation [69]. CRN effectors translocation is dependent on the N-terminal LFLAK domain but the exact mechanism of translocation is unknown [56] Within plant cells, hosttranslocated effectors target different subcellular compartments where they interfere with plant processes. Some effectors contain sorting signals like nuclear localization signals or transit peptide-like sequences that target the protein to different organelles within the plant cell [56, 253, 254, 255]. Other effectors lack these sorting signal and possibly piggy-back on interacting host proteins that are targeted to specific compartments.

We visualized the subcellular localization of the first eight candidate host-translocated effectors of Pfs 1 by detecting fluorescent effector-fusion proteins in $N$. benthamiana epidermal cells using confocal microscopy. RxLR effectors Pfs1|3869, Pfs1|1800, Pfs1|10763 and CRN effector Pfs1|9071 localized to the nucleus or showed a nucleocytoplasmic localization. Studies in other species showed that the nucleus and cytoplasm are common sites of effector function. All CRNs studied so far and the majority of RxLR effectors of other oomycete species localized in the nucleus or cytoplasm [56, 240, 256]. A multitude of subnuclear localizations are reported for both RxLR and CRN effectors, something we also observe in our small subset of effectors. In the individual subcellular localization experiments we found Pfs1|3869, Pfs1|1800 and Pfs1|10763 in the nucleoplasm and Pfs1|10763 in large nuclear speckles. Additionally, in co-expression experiments, candidate effectors were observed in small nuclear speckles (Pfs1|6786), the nuclear membrane and nucleolus (Pfs1|3172). The other effectors localized to the ER (Pfs1|3172) and 
PS (Pfs1|6786 and Pfs1|7215, Pfs1|9976). Co-expression of the fusion proteins with molecular markers for ER, Golgi, plastoglobuli, lipid bodies, autophagosomes or vacuoles should be performed to confirm the origin of the PS. Remarkably, candidate host-translocated CRN effector Pfs1|9976 did only localize to the nucleus when co-expressed with FBN1a, but not in experiments expressing only the effector - where it was observed in PS.

The subcellular localization observed for seven out of eight of the effectors did not match the predicted DeepLoc localizations. The DeepLoc predictions are based on a neural network-trained on experimentally verified eukaryotic proteins derived from Uniprot. The prediction does not explicitly take into account known sorting signals like signal or transit peptides. In studies of other oomycete species, poor prediction of subcellular localization was also encountered for host-translocated effectors [148]. The latter is addressed by LOCALIZER, a machine learning method that uses a sliding window approach to predict NLS, and chloroplast/mitochondrial transit peptides [257]. As opposed to other prediction methods like TargetP [258], LOCALIZER does not assume that a transit peptides starts at the first residue after the signal peptide. This likely improves the prediction because it was shown that signal and transit peptides are in some cases separated by so-called 'pro-domains' of varying length [257, 259]. LOCALIZER predicted nuclear localization for Pfs1|9976 and nuclear and chloroplast localization for Pfs1|10763, which is not in line with the observed localization in the individual expression experiments. However, Pfs1|9976 relocalized to the nucleus upon coexpression with FBN1a. The prediction of localization of effector proteins is likely complicated by translocation from one organism to another and the ability of the usually small effector proteins to co-migrate through the cells with interacting proteins. Additionally, the signal peptide is likely removed during translocation and it is unknown whether the $\mathrm{N}$-terminus is further processed during transport [260].

Within plant cells, host-translocated effectors interact with plant proteins to manipulate host processes to the benefit of the oomycete. A prerequisite for interaction is that the effector and interactor reside in 
the same subcellular compartment. We found that twelve of the fourteen Arabidopsis and all of the thirteen spinach orthologs localized at least partially to the same subcellular compartments as the effector that was used as bait to identify them in the $\mathrm{Y} 2 \mathrm{H}$ screen, supporting their proposed interaction (Chapter 3). Interestingly, the fluorescent signal of interactor-fusion proteins that were barely visible upon individual transient expression, stabilized upon co-expression with the candidate effector. This suggests that the presence of, or interaction with the effector stabilizes the plant proteins. Additionally, some interactor proteins and two candidate host-translocated effectors (Pfs1|6786, Pfs1|9976) showed a different subcellular localization in the co-expression compared to individual expression experiments.

It is tempting to speculate and hypothesize on the putative processes that are affected by the Pfs effectors. The plant proteins targeted by the candidate host-translocated Pfs 1 effectors can be roughly divided into three functional classes, based on their alleged molecular function. The functional classes are; cellular programming, metabolic homeostasis and "other" or "unknown" for proteins that have no clear possible link to immunity. Manipulation of cellular programming

4 can be accomplished at different levels; directly through manipulation of transcription or posttranscriptional processes or indirectly through manipulation of signaling pathways, e.g. in hormonal or immune signaling (Chapter 1). Below, a number of effector interactions and their possible impact on cellular processes are freely discussed (sometimes providing speculative ideas).

\section{Cellular programming - manipulation of hormone and immune signaling}

Candidate CRN effector Pfs1|9976 was found to interact with FBN1a, a fibrillin protein that is found in plastids, primarily in lipid particles called plastoglobuli [261]. Plastoglobuli have a membrane lipid monolayer and contain lipids and proteins [262]. Their exact biological function is unclear, but they were observed to grow during developmental processes 
and stress responses [262]. Plastids, particularly chloroplasts are sites of synthesis of phytohormones (jasmonic and salicylic acid), defense compounds, reactive oxygen species and nitric oxide and thus play an important role in plant immunity [263].

We observed the FBN1a fusion protein in PS that possibly represent plastoglobuli. The spinach ortholog of FBN1a was also observed in the ER and nucleus. Localization of fibrillin in the nucleus was reported before (Table 1). FBN1a orthologs in species other than Arabidopsis were found to be differentially expressed upon pathogen invasion. FBN1a orthologs were upregulated upon infection with obligate biotrophic as well as necrotic fungal and bacterial pathogens in cucumber, tomato and grape [264, 265]. RNA silencing of FBN1a ortholog LeCHRC (Lycopersicon esculentum carotenoid-associated protein) in tomato plants increased susceptibility to the necrotrophic fungus Botrytis cinerea, while RNAi lines overexpression LeCHRC (sense) were more resistant to infection [264]. FBN1a can be considered a common effector target as it was found to interact with effectors from bacterial, fungal and oomycete species (Chapter 3, [219]). Interestingly, a role for FBN1a was implicated in jasmonic acid biosynthesis. The application of exogenous methyl jasmonate was shown to abolish the effect of knock-down of FBN12. Also, FBN1a was found to co-localize with the biosynthetic enzyme allene oxide synthase in plastoglobules [266]. Jasmonic acid antagonizes salicylic acid-mediated immune responses against biotrophic pathogens [267]. We observed re-localization of FBN1a and Pfs1|9971 from PS to the nucleoplasm upon co-expression with candidate host-translocated CRN effector Pfs1|9971. Interestingly, the effector nor de interactor were observed in the nucleus when expressed individually. Given the published information, a re-localization of FBN1a away from plastoglobuli does not provide a clear benefit to Pfs. If FBN1a positively regulates jasmonic acid synthesis than re-location results in lower levels of the hormone. Additional factors like timing, co-expression with other effectors and cell type targeting may affect the outcome of binding of FBN1a by Pfs1|9971. Alternatively, translocation of proteins from chloroplast to the nucleus 
is observed during retrograde signaling. Chloroplasts may function as environmental sensors that mediate stress, like pathogen infection, by signaling that leads to alternative regulation of genes [263]. However, other than translocation of FBN1a to the nucleus upon co-expression with CRN effector Pfs1|9976 there is no indication of such a role for FBN1a.

Candidate host-translocated RxLR effector Pfs1|7215 interacts with LSU1. As its name implies, LSU1 proteins are upregulated in response to S-deficiency [268]. Mutant Isu1 plants produce reduced amounts of reactive oxygen species (ROS) resulting in impaired stomatal closure in response to low sulphur conditions [269]. LSU1 is an effector target of evolutionary diverse pathogens, suggesting that the protein has a yet unknown role in plant immunity [206, 219]. Possibly effectors manipulate LSU1 to alter ROS production, and hence the redox state of the cell, which serves as a stress signal and plays an activating role in plant immunity [270]. Plastid localized bacterial effectors were shown to sabotage the activating interaction between LSU1 and the superoxide dismutase FSD2, hampering the conversion of $\mathrm{O}_{2}$ to $\mathrm{H}_{2} \mathrm{O}_{2}$. Pseudomonas syringae pv. tomato (Pst) DC3000 infection resulted in reduced chloroplast localization of LSU1 during low sulfur (S) stress. Plants that lacked LSU1 4 expression showed a moderately enhanced susceptibility to Pst under low S-stress but not under standard conditions [269]. We observed the LSU1 fusion protein primarily in the cytoplasm and in PS. Spinach ortholog Sp022403 was only observed in PS. Both proteins co-localized with Pfs1|7215 in PS and LSU1 also in the nucleo-cytoplasm. The colocalization between Pfs1|7215 and LSU1 suggests that Pfs, through effector Pfs1|7215, could interfere with ROS production and stomatal closure as well. 


\section{Cellular programming - transcriptional manipulation}

Pfs $1 \mid 3172$ is a candidate RxLR effector that has a C-terminal transmembrane domain and interacted with two membrane-bound NAC transcription factors in the $\mathrm{Y} 2 \mathrm{H}$ screens. The transcription factors have a highly conserved N-terminal DNA-binding NAC domain and a more variable C-terminal transcriptional regulation domain [271] More than 100 NAC transcription factor genes are present in the genome of Arabidopsis. At least nine of them have been linked to different aspects of immunity, like hormone signaling and stomatal closure [272]. The identification of AtNAC014 and AtNAC078 as interactors of Pfs1|3172 may add these two candidates to this list. The transmembrane domain anchors the transcription factors to the plasma- or ER-membrane rendering it inactive as a transcription factor [273]. The membrane-bound NACs are proteolytically cleaved upon developmental and environmental cues, enabling the NAC transcription factor to move into the nucleus to regulate gene transcription $[86,169]$.

We observed co-localization of the host-translocated effector and the (truncated) NAC014 transcription factor in the ER, PS and potentially in the nucleus. The full-length spinach and Arabidopsis NACs stabilized upon co-expression with the effector and were observed in the ER (NAC014), PS and nucleus/cytoplasm (Spo22390, NAC078). The observed subcellular localization patterns resemble that of known oomycete effectors that interact with tail anchored NAC transcription factors of lettuce (LsNAC069) (Lactuca sativa) and potato (StNTP1, StNTP2) (Solanum tuberosum). These effectors were found to prevent Phytophthora culture filtrate-induced re-localization of the NAC transcription factors to the nucleus. $N$. benthamiana plants in which NbNTP1, NbNTP2 were silenced, showed increased susceptible to $P$. infestans infection and silencing of the Pi03192 effector gene reduced virulence of $P$. infestans [86]. Higher susceptibility against the corresponding oomycete $B$. lactucae was not observed in LsNAC069silenced $L$. sativa transformants [169]. The $B$. lactucae, $P$. infestans and in the current study also the Pfs host-translocated effectors, all interact 
with tail-anchored NAC transcription factors from species other than their host, (i.e., P. infestans Pi03192 interacts with lettuce NACs, Bremia effectors interact with Arabidopsis and potato NACs and Pfs effectors interact with Arabidopsis NACS) suggesting that tail-anchored NAC transcription factors are conserved oomycete effector targets.

$\mathrm{HBI} 1$ is a bHLH transcription factor that plays an important role in the growth-defense trade-off in plants as it positively regulates leaf cell expansion and negatively regulates ROS production upon exposure to PAMPs [226, 274]. HBI1 interacted with candidate RxLR effector Pfs1|6786. Interestingly, the subcellular localization of Pfs $1 \mid 6786$ changed from predominantly PS to predominantly nuclear upon co-expression with HBI1, Spo6524 (HBI1 ortholog), AHAS, and RAF2. We found that the $\mathrm{HBI} 1$ protein is stabilized in the nucleus by the presence of effector Pfs1|6786. When expressed individually, the HBI1 fusion protein was hard to observe due to low signal and if visible was observed in the cytosol and nucleus. Stabilization of HBI1 could be beneficial for Pfs 1 , as HBI1 is a negative regulator of PAMP-triggered immunity. Despite its clear role in basal immunity, $\mathrm{HBI} 1$ is not targeted by many pathogen effectors, in fact, to our knowledge Pfs $1 \mid 6786$ is the first effector reported to target and 4 stabilize HBI1. The spinach ortholog of HBI1, Spo06524 shows a similar, nuclear distribution when individually expressed but is in small PS, just like the effector when co-expressed. One should note that Spo06524 is not a reciprocal best hit of $\mathrm{HBI} 1$ and may have a different function.

\section{Cellular programming - posttranscriptional manipulation}

RxLR effector Pfs1|1800 was found to interact with a protein (At2g19390) of unknown function that is rich in serine and arginine dipeptides. Serinearginine (SR)-rich proteins are known to be involved in alternative splicing of mRNAs [275] However, the plant interactor of Pfs1|1800 lacks RNA binding domains that characterize known SR proteins. In At2g19390, the SR dipeptides are distributed throughout the protein, rather than at the C-terminus in SR-rich proteins with known splicing activity [276]. 
We found that At2g19390 was localized to small nuclear speckles while ortholog Spo24486, localizes to large nuclear speckles. Nuclear speckles are sites of storage and assembly of splicing factors that are observed near active transcription sites. Active cells possess many small speckles, whereas large speckles are associated with inhibition of transcription and splicing [249]. Upon co-expression with effector Pfs $1 \mid 1800$, the subnuclear localization of the At2g19390 protein changes from speckles to the nucleoplasm. Co-expression of the spinach ortholog and effector leads to their co-localization in large nuclear speckles. It will be interesting to test if the effector interferes with RNA processing in the plant cell nucleus.

RxLR effector Pfs1|10763 interacts with the At3g11930 protein that has a universal stress protein A domain (USPA). USPA is an ancient domain that was likely present in the last universal common ancestor of all extant life [277]. Bacterial USPA proteins mediate cell survival under a variety of abiotic stresses [278]. The Arabidopsis genome encodes 44 proteins with USP domains [234], of which At3g53990 was shown to exhibit a redox-dependent chaperone function that enhances plant tolerance to heat shock and oxidative stress [279]. Comparative analysis showed that the interactor USPA has conserved ATP binding residues [234], but functional data on this protein is lacking. Co-expression with Pfs1|10763 resulted in a change of subcellular localization of At3g11930 from nucleo-cytoplasmic to nuclear, where it is present in large nuclear speckles. The spinach ortholog Spo22595 was unstable but showed a clear signal from the nucleoplasm in the presence of the effector, which is also detected there. The altered localization of the plant interactors when the effector is present prompts further research into the function At3g11930 during downy mildew infection and its role as a potential effector target. 


\section{Manipulation of metabolic homeostasis}

AHAS or Acetohydroxyacid synthase is a protein that catalyzes the first step in the synthesis of the branched-chain amino acids valine, leucine and isoleucine [280]. A study in tomato showed that a near-isogenic line carrying the Ol-1 resistance locus expresses an AHAS homolog that is involved in resistance to the powdery mildew fungus Oidium neolycopersici. The resistance seems to be the result of altered AHASmediated amino acid homeostasis, i.e. altered levels of branched-chain amino acids in cells, rather than a basal or resistance gene-based defense mechanism [230]. Tomato plants treated with the AHAS-inhibiting herbicide chlorsulfuron were more susceptibility to Oidium neolycopersici infection. Chlorsulfuron treatment was shown to reduce levels of valine and leucine in soybean suspension cultures. The observed plastid localization when individually expressed is in line with published research [280]. We observed a shift of the AHAS-fusion protein to the nucleoplasm when co-expressed with Pfs1|6786, while it was still visible in plastids/ PS. The outcome of interference of Pfs1|6786 with the synthesis of branched-chain amino acids and an effect of that on Pfs virulence is hard to predict, because amino acid synthesis requires multiple proteins and is negatively regulated by the amino acids produced [230]. Also, the plant needs the amino acids for its own metabolism and functioning. The spinach ortholog Spo20077 was observed in the cytoplasm and PS but not in in the plastid lumen. Co-expression did not alter the localization of Pfs1|6786 nor Spo20077.

RAF2 is a Rubisco assembly chaperone that was first identified in maize. RAF2 associates with the Rubisco small subunit and possibly facilitates assembly with the large subunit by releasing the small subunit from the chaperone complex [224] Surprisingly we observed RAF2 primarily in the nucleus rather than in chloroplasts, both in the individual and co-expression experiments where the presence of RAF2 appears to direct Pfs1|6786 to the nucleus as well. Likely, the N-terminal fluorescent tag prohibited the transit into the chloroplasts. The signal is not only in the nucleoplasm, which could occur by simple diffusion but also strongly 
in nuclear speckles - it is possible that RAF2 resides in the nucleus as well. Localization was observed in the majority of the cells $(>10)$ and a band of the correct molecular weight (of the protein including the transit peptide) was observed on the western blot. The localization of the $\mathrm{N}$-terminal fusion protein to the nucleus, therefore, seems genuine. It will be important to look into mesophyll cells that have more chloroplasts, using C-terminally fused fluorescent proteins, to see whether the protein and effector localize to chloroplasts as well.

The final metabolism-related interactor protein is NAGK, a protein that is involved in the synthesis of arginine in chloroplasts. Under high nitrogen conditions, NAGK is bound by the plastid signal transduction protein PII to induce arginine synthesis. Binding between these proteins is reduced when little nitrogen is available or when arginine levels increase [281]. Arginine is the substrate for production of nitric oxide (NO) by nitrogen oxide synthase NOS1 [282]. PAMP-treatment in Arabidopsis plants was shown to trigger a NO-burst which is emergingly recognized as a plant PAMP-triggered immunity reaction, analogous to that in animals [282]. Plants with a mutated AtNOS1 are not able to convert arginine to NO and were more susceptible to Pseudomonas syringae pv. tomato DC3000 [282]. We observed NAGK in the ER and PS. The protein was expressed with an $\mathrm{N}$-terminal tag that likely prohibits the entry of the fusion protein into the chloroplast, although the PS structures may represent chloroplast plastoglobuli. When co-localized with the effector, NAGK localizes to PS of different sizes and no signal is observed from the ER. The spinach ortholog stabilized upon co-expression with Pfs1|3869 and was observed in PS, nucleoplasm and nuclear speckles. 


\section{Other effector-interacting proteins}

Little is known about the interactor At5g56100 that is annotated as 'glycine-rich/oleosin'. Oleosins are proteins that locate on the surface of lipid bodies that store lipids in seeds to facilitate their germination and seedling growth [283]. However, this protein is oleosin-like and it is not known whether it localizes to these bodies. We observed the protein in the ER and in PS in epidermal cells. It co-localizes with the candidate host-translocated CRN effector in the ER, PS and cytoplasm. Despite its limited similarity, the subcellular localization pattern of spinach ortholog Spo12707 is similar to that of At5g56100. Little is known about the mode of action of CRN effectors. A sparse example of CRN-interacting proteins is described for $P$. sojae effectors CRN115 and CRN63 that were shown to interact with plant catalases to regulate $\mathrm{H}_{2} \mathrm{O}_{2}$ production elicited by CRN63 and overcome host immunity [103] .

The final protein that was found to co-localize with an effector is At3g13800, a protein with a Metallo-beta-lactamase (IPR001279) domain of unknown function. At3g13800 and its spinach ortholog co-localize with the membrane bound Pfs $1 \mid 3172$ effector in the ER and in PS. Further research should focus on the molecular role of At3g13800 and its effect on plant susceptibility and pathogen infection. 


\section{Conclusion}

In this chapter we visualized the subcellular localization of the first eight candidate host-translocated effectors of Pfs. We found that in most cases the effectors co-localize with their putative interacting plant proteins and their identified spinach orthologs. Co-expression in some cases let to stabilization and re-localization of either or both of the interacting proteins within plant cells. Future research should validate the interaction between the proteins in planta and set out to understand the molecular roles of the plant proteins in immunity and the ways the host-translocated effectors manipulate these functions.

\section{Methods}

\section{Reciprocal blast analysis of candidate spinach orthologs}

A reciprocal blastP search was performed to identify the spinach protein orthologs of the Arabidopsis interactor proteins. Arabidopsis and spinach protein Blast libraries were created using TAIR10_pep_20110103_ representative_gene_model" (arabidopsis.org) and 'spinach protein sequences V1' (http://www.spinachbase.org/) in Geneious 9.1 .8 (https:// www.geneious.com) [284]. The Arabidopsis interactor proteins were used as the query in the first search, the best spinach protein hits (E-value $<1.0 \mathrm{e}-05$ ) were considered candidate protein orthologs. The reciprocal blast was performed using the significant spinach hits on the library of Arabidopsis proteins. In case no reciprocal best hit was identified, a protein tree was created to clarify possible multiple-to-one or multipleto-multiple orthology. For each Arabidopsis interactor all significant reciprocal BlastP hits were aligned (MUSCLE [285] and Neighbor-Joining trees (Tamura-Nei distance model) were created using standard settings (Geneious 9.1.8). Trees were visualized using iTOL [212]. 


\section{Prediction of subcellular localization}

The subcellular localization of candidate effectors without their signal peptides was predicted using LOCALIZER 1.0.4 [257] and Deeploc 1.0 [286] using standard settings. The subcellular localization of interacting Arabidopsis proteins was retrieved from Suba4 [248].

\section{Cloning}

mRNA was isolated from infected Arabidopsis Col-0 and spinach Viroflay plants using the Qiagen mRNA isolation kit according to protocol. cDNA was generated using Maxima $\mathrm{H}$ Minus Reverse Transcriptase and oligo(dT) primers at standard settings. Primers were designed to include attachment sites to aid Gateway BP or TOPO cloning (Table S2). Effectors and interactors were amplified from cDNA of infected plants and cloned into a pGEM-T-Easy using BP clonase according to the Gateway protocol or in the pENTR/D-TOPO vector according to the TOPO cloning kit. Vectors were transformed into $E$. coli $\mathrm{DH} 5 \alpha$ for selection using ampicillin as a selection marker. Correct insertion of the coding sequences into the vector was verified by Sanger sequencing (Macrogen). The entry vectors were used to transfer the genes of interest to YFP- and CFP-containing expression vectors from the pB7WG2 vector series for expression from the $35 \mathrm{~S}$ promoter of fluorescent fusion proteins in planta [235].

\section{Transformation of Agrobacterium for expression of fusion proteins in planta}

The pB7WG2 series expression vectors were transformed into Agrobacterium tumefaciens strain AGL1 using heat shock to allow for large scale transformation of plasmids. Cells were grown in $200 \mathrm{ml}$ of LB with $25 \mu \mathrm{g} / \mathrm{ml}$ rifampicin and $15 \mathrm{ug} / \mathrm{ml}$ tetracycline at $28^{\circ} \mathrm{C}$ overnight until the cells were in the exponential growth phase (OD600 $=0.5-0.8)$. Cell were chilled on ice for 10 minutes and collected at $4700 \mathrm{rpm}$ at $4^{\circ} \mathrm{C}$. Supernatant was discarded and the cells were suspended in $2 \mathrm{ml}$ of ice cold $20 \mathrm{mM} \mathrm{CaCl} 2$ and aliquoted in $100 \mu \mathrm{l}$ samples that were snap frozen in liquid nitrogen. To transform the Agrobacterium cells, between 100 
and $1000 \mathrm{ng}$ of plasmid was added to each thawed aliquot which was then again snap frozen in liquid nitrogen for 5 minutes. Cells were heat shocked for 5 minutes at $37^{\circ} \mathrm{C}$ degrees, transferred to a $15 \mathrm{ml}$ tube with $1 \mathrm{ml}$ of LB and incubated for 1 hour at $28^{\circ} \mathrm{C}$ while shaking. The sample was plated on LB supplemented with the appropriate antibiotics at $28^{\circ} \mathrm{C}$ to form colonies within two days. The insert size of the transformed Agrobacterium colonies was verified using colony PCR with plasmid specific primers.

\section{Transient expression in $\mathbf{N}$. benthamiana}

$N$. benthamiana plants were grown in long day conditions (16 hours of light at $22{ }^{\circ} \mathrm{C}$ and 8 hours of darkness at $20^{\circ} \mathrm{C}$ ). Agrobacterium with the desired plasmid was grown overnight at $28^{\circ} \mathrm{C}$ in $\mathrm{LB}+50 \mathrm{nM}$ spectinomycin (spec), $50 \mathrm{nM}$ carbenicillin (carb) \& $20 \mathrm{nM}$ rifampicin (rif). Cells were pelleted and resuspended into $7.5 \mathrm{ml}$ of induction medium (1x M9 salts, $1 \%$ glucose) + appropriate antibiotics (spec, carb, rif) and incubated for 3 hours at $28^{\circ} \mathrm{C}$. Cells were pelleted and resuspended in infiltration medium (0.5x Murashige \& Skoog salts, $10 \mathrm{mM}$ 2-(N-Morpholino) ethanesulfonic acid sodium salt (MES), $0.5 \%$ sucrose, $0.5 \%$ fructose and $150 \mu \mathrm{M}$ of acetosyringone). Three leaves of 3 week-old short day grown spinach or five week-old $N$. benthamiana plants were infiltrated with Agrobacterium at an OD600 of 0.6. Effectors and targets were expressed separately and in combination, with the OD600 kept at 0.6 for the mixtures. The infiltrated plants were grown for 48 hours before examination under the confocal microscope. The number of technical replicates and the number of transformed cells observed per construct are indicated in Table S2. 


\section{Confocal microscopy}

Expression of fluorescent proteins was assessed under a Zeiss $₫$ LSM700 confocal microscope using the $63 x$ objective with oil emersion (Objective Plan-Apochromat 63x/1.40 Oil DIC). Excitation for CFP was set at 405 and YFP at $488 \mathrm{~nm}$. The emission wavelength for both CFP and YFP was set at $523 \mathrm{~nm}$ with a detection limit of 493-550 nm. Images were analyzed with ZEN lite v2.3 software (Zeiss microscopy) and the overview figures were created using Photoshop v. 1.9. Table S3 lists the number of technical replicates and numbers of cells visualized per protein and per host-translocated effector-plant interactor combination.

\section{Western blot detection of effector protein and plant protein fusions}

Three leaf punches $(6 \mathrm{~mm})$ of the infiltrated $N$. benthamiana plants used in the confocal assays were snap frozen in liquid nitrogen and grinded using a mortar and pestle. $100 \mu$ of lysis buffer $(10 \mathrm{mM}$ Tris- $\mathrm{HCl} \mathrm{pH}$ 7.6, $150 \mathrm{mM} \mathrm{NaCl}, 0.5 \mathrm{mM}$, EDTA, 0.25\% (v/v) Triton-X 100, 0.25\% (v/v) Tween-20, $5 \mathrm{mM}$ DTT, 4\% (w/v) polyvinylpyrrolidone) was added to the grinded sample and incubated at $4^{\circ} \mathrm{C}$ for 30 minutes while shaking. After incubation the sample was centrifuged $\left(4^{\circ} \mathrm{C}\right.$ for 30 minutes, $\left.7000 \mathrm{rpm}\right)$ and the supernatant for another 10 minutes under the same conditions. Laemlli buffer $(33,3 \mu \mathrm{l})$ was added and the sample was cooked for 10 minutes at $95^{\circ} \mathrm{C}$ and centrifuged for 0 minutes at $6600 \mathrm{rpm}$. Samples were loaded to a pre-cast Invitrogen Bolt Bis-Tris Plus gradient gel $4-12 \%$. Gel loading and western blotting was performed according to standard protocol. Poncou staining was performed to validate the total level of the proteins. A horse radish peroxidase conjugated GFP antibody (Chromotek) was used to visualize the fusion proteins in the sample. 


\section{Acknowledgements}

I wish to thank the students that did the majority of the cloning; Martijn Breeuwsma and Kim Nijhof. Thank you, Kim Baremans, Frederike Bijlmer, Zuzanna Stępień, Joyce Elberse and Annemiek Andel for your work related to this chapter. Additionally, we thank TKI T\&U (Topsector Tuinbouw \& Uitgangsmaterialen), Enza Zaden, Rijk Zwaan, Syngenta and Pop Vriend for funding the project. 


\section{Supplemental tables}

Table S1. Western blot using an antibody against the fluorescent protein for detection of intact fusion proteins in Nicotiana benthamiana leaves.

\begin{tabular}{|c|c|c|c|}
\hline & Protein & Size (KDa) & observed in western blot \\
\hline \multirow{16}{*}{$\begin{array}{l}\frac{0}{0} \\
\frac{0}{0} \\
\frac{0}{0} \\
\frac{0}{\pi} \\
\frac{\pi}{\alpha}\end{array}$} & AT1G33060 (1) & 54 & no \\
\hline & AT1G33060_trunc & 53 & yes \\
\hline & AT1G55190 & 21 & no \\
\hline & AT2G18300 (1) & 41 & no \\
\hline & AT2G19390 (1) & 133 & no \\
\hline & AT3G11930 (2) & 25 & yes \\
\hline & AT3G13720 & 21 & no \\
\hline & AT3G13800 (1) & 40 & no \\
\hline & AT3G48560 & 73 & yes \\
\hline & AT3G49580 (1) & 11 & yes \\
\hline & AT3G57560 & 37 & no \\
\hline & AT4G04020 & 35 & yes \\
\hline & AT4G35110 (3) & 45 & yes (low amount) \\
\hline & AT5G04410 & 19 & no \\
\hline & AT5G51110 (1) & 24 & yes \\
\hline & AT5G56100 & 16 & yes \\
\hline \multirow{15}{*}{ 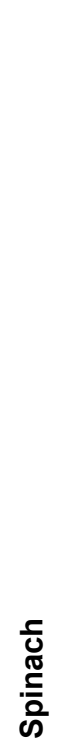 } & Spo22390 & 22 & no \\
\hline & Spo22390 (variant) & 22 & yes \\
\hline & Spo20420* & 22 & yes \\
\hline & Spo06524 & 52 & no \\
\hline & Spo24468 & 137 & no \\
\hline & Spo22595 & 23 & no \\
\hline & Spo20405 & 40 & yes \\
\hline & Spo20077 & 73 & yes \\
\hline & Spo22402 & 14 & yes \\
\hline & Spo15075 & 44 & yes \\
\hline & Spo20112 & 35 & yes \\
\hline & Spo18795 & 40 & not available \\
\hline & Spo16524 & 19 & no \\
\hline & Spo14154 & 24 & yes \\
\hline & Spo12707 & 16 & no \\
\hline
\end{tabular}




\section{Table S1 continued}

\begin{tabular}{|c|c|c|c|}
\hline 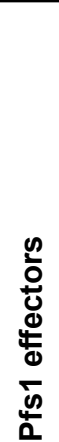 & \begin{tabular}{|l|} 
pfs1|9071 \\
pfs1|9976 \\
pfs1|1800 \\
pfs1|10763 \\
pfs1|6786 \\
pfs1|3172 \\
pfs1|10492 \\
pfs1|3869 \\
pfs117215
\end{tabular} & $\begin{array}{l}11 \\
69 \\
20 \\
48 \\
41 \\
18 \\
80 \\
20 \\
19\end{array}$ & $\begin{array}{l}\text { yes } \\
\text { no } \\
\text { yes } \\
\text { yes } \\
\text { yes } \\
\text { yes } \\
\text { not available } \\
\text { yes } \\
\text { yes }\end{array}$ \\
\hline
\end{tabular}


Table S2. Overview of the number of technical replicates and the number of transformed cells observed in the(co-)expression experiments of fluorescent fusion proteins in $\mathbf{N}$. benthamiana leaves.

\begin{tabular}{|c|c|c|c|c|c|}
\hline Gene code & Protein name & $\begin{array}{l}\text { Individu } \\
\text { Techn. } \\
\text { repl. }\end{array}$ & $\begin{array}{l}\text { al expression } \\
\text { Cumulative \# } \\
\text { cells }\end{array}$ & $\begin{array}{l}\text { Co-expressi } \\
\text { Techn. repl. }\end{array}$ & $\begin{array}{l}\text { Cumulative } \\
\text { \# cells }\end{array}$ \\
\hline At1g33060 & NAC014 & 2 & 3 & 1 & 8 \\
\hline At1g55190 & PRA1.F2 & 1 & 9 & 1 & 4 \\
\hline At2g18300 & $\mathrm{HBI} 1$ & 5 & 4 & 1 & 2 \\
\hline At2g19390 & S-R rich & 1 & 9 & 1 & 5 \\
\hline At3g11930 & USPA & 2 & 4 & 1 & 7 \\
\hline At3g13720 & PRA1.F3 & 1 & 8 & 1 & 5 \\
\hline At3g13800 & AT3G13800 & 1 & 5 & 1 & 8 \\
\hline At3g48560 & AHAS & 1 & 7 & 2 & 9 \\
\hline At3g49580 & LSU1 & 3 & 13 & 3 & 17 \\
\hline At3g57560 & NAGK & 1 & 6 & 1 & 9 \\
\hline At4g04020 & FBN1a & 1 & 7 & 1 & 7 \\
\hline At5g04410 & NAC078 & 2 & 3 & 2 & 9 \\
\hline At5g51110 & RAF2 & 1 & 13 & 1 & 10 \\
\hline At5g56100 & Glycin rich & 3 & 16 & 2 & 20 \\
\hline $\begin{array}{l}\text { AT1G33060_ } \\
\text { trunc. }\end{array}$ & NAC014 trunc. & 2 & 10 & 1 & 4 \\
\hline
\end{tabular}


Table S2. continued

\begin{tabular}{|c|c|c|c|c|c|}
\hline Gene code & Protein name & $\begin{array}{l}\text { Individue } \\
\text { Techn. } \\
\text { repl. }\end{array}$ & $\begin{array}{l}\text { al expression } \\
\text { Cumulative \# } \\
\text { cells }\end{array}$ & $\begin{array}{l}\text { Co-expressic } \\
\text { Techn. repl. }\end{array}$ & $\begin{array}{l}\text { Cumulative } \\
\text { \# cells }\end{array}$ \\
\hline Spo22390 & AT1G33060 & 2 & 2 & 1 & 3 \\
\hline Spo20420* & AT1G55190 & 2 & 7 & 1 & 6 \\
\hline Spo06524 & AT2G18300 & 2 & 5 & 1 & 7 \\
\hline Spo24468 & AT2G19390 & 2 & 7 & 3 & 10 \\
\hline Spo22595 & AT3G11930 & 2 & 5 & 1 & 7 \\
\hline Spo20405 & АT3G13800 & 1 & 4 & 1 & 6 \\
\hline Spo20077 & AT3G48560 & 1 & 4 & 2 & 8 \\
\hline Spo22402 & AT3G49580 & 2 & 16 & 3 & 16 \\
\hline Spo15075 & AT3G57560 & 2 & 2 & 1 & 8 \\
\hline Spo20112 & AT4G04020 & 1 & 7 & 1 & 5 \\
\hline Spo16524 & AT5G04410 & 2 & 3 & 1 & 9 \\
\hline Spo14154 & AT5G51110 & 1 & 4 & 1 & 8 \\
\hline Spo12707 & AT5G56100 & 3 & 9 & 3 & 10 \\
\hline Pfs1|3172 & & 2 & 10 & & \\
\hline Pfs1|9071 & & 3 & 13 & & \\
\hline Pfs1|6786 & & 1 & 5 & & \\
\hline Pfs1|1800 & & 2 & 13 & & \\
\hline pfs1|10763 & & 2 & 15 & & \\
\hline Pfs1|7215 & & 4 & 18 & & \\
\hline Pfs1|3869 & & 1 & 3 & & \\
\hline Pfs1|9976 & & 2 & 7 & & \\
\hline
\end{tabular}




\section{Supplemental figures}

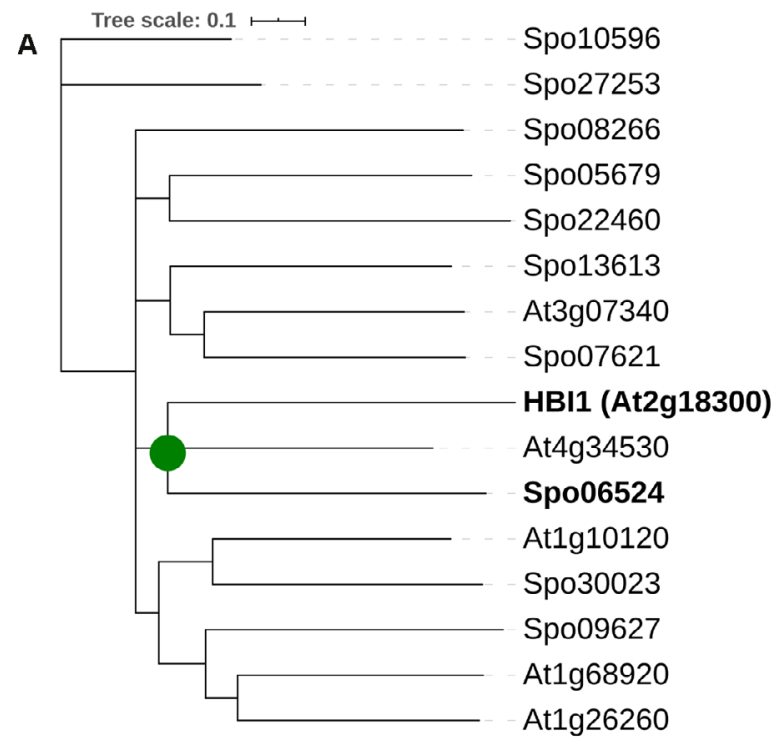

B
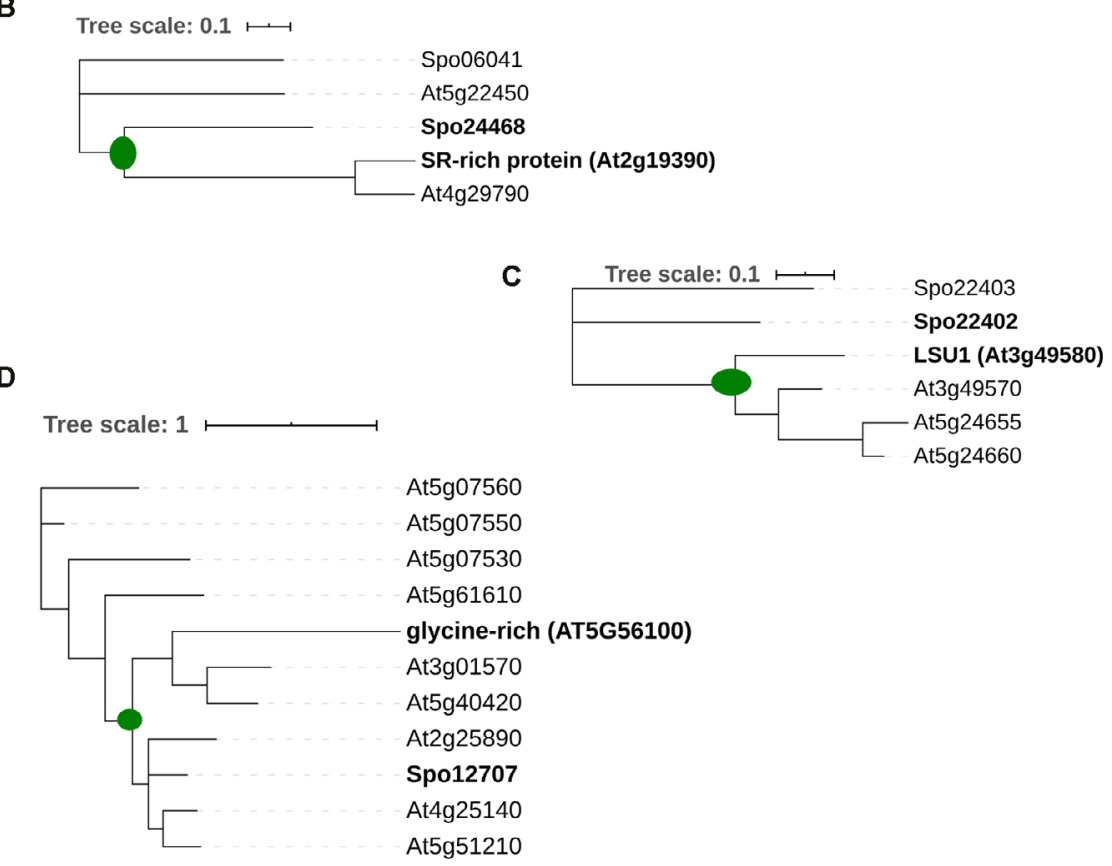

Figure S1. Protein tree of Arabidopsis effector-interactors, their protein paralogs and spinach orthologs.

Green dots represent the last shared branch between the spinach and Arabidopsis protein. 
Figure S2. Protein alignments of Arabidopsis interactor proteins and the selected spinach Sp75 orthologs

Available at:

https://doi.org/10.17026/dans-zey-8aec

\section{Expression in}

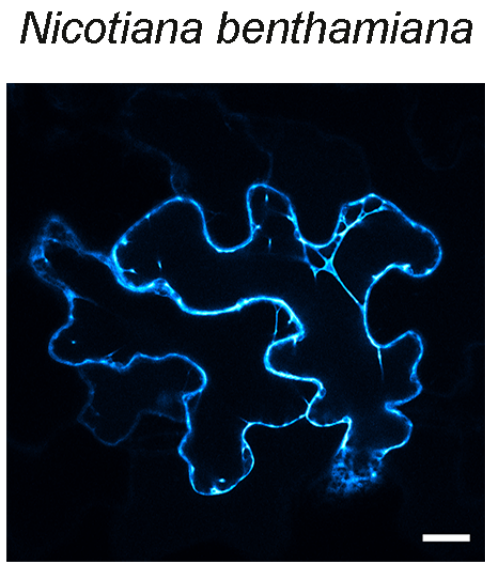

Spinach race Viroflay

\section{At3g49580; ER, Golgi}
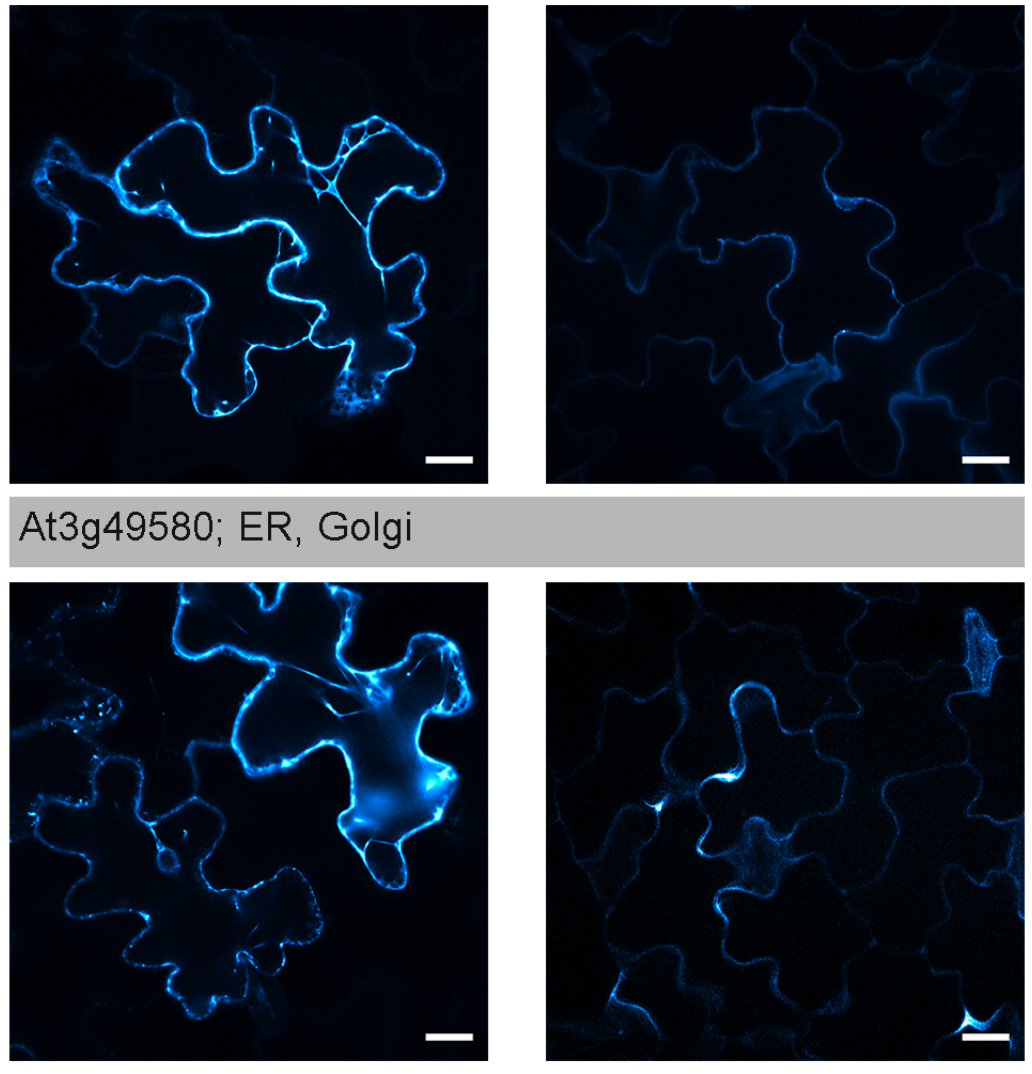

\section{Spo12707; Golgi, ER, cytosol}

Figure S3. Expression of fusion proteins in $\mathbf{N}$. benthamiana (left) and spinach variety Viroflay (right).

Each line represents the same protein in each of the plant species. Pictures are taken at 3 days post infection. Scale bars are $20 \mu \mathrm{m}$. 




$$
\text { Spinach ortholog }
$$


Spo20420; ER, PS, nucl.,cyto.
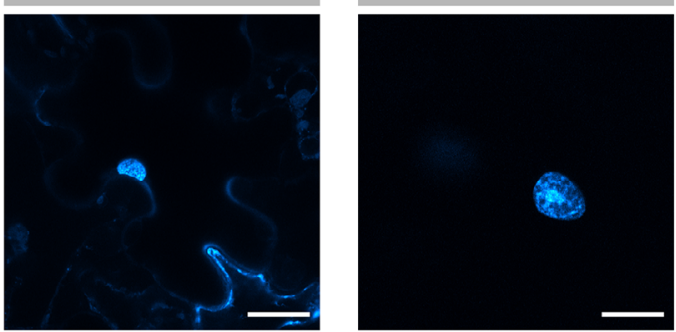

$\mathrm{HBI} 1$; NS

Spo06524; NS, nucleolus

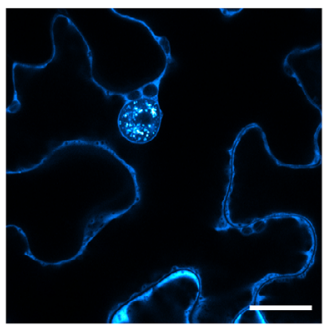

At2g19390; NS, cyt., ER

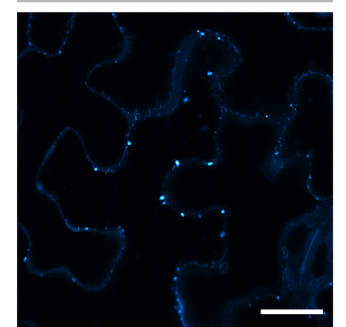

Spo24468; PS, nucleus

Figure S4. Subcellular localization of Arabidopsis interactors and their spinach orthologs in $N$. benthamiana.

Plant interactors fused to CFP (N-terminus) were produced in N. benthamiana. AHAS and ortholog Sp020077 were fused at its C- terminus to CFP. Images were taken $\sim 48$ to 72 hours after infiltration. Scale bars $=20 \mu \mathrm{m}$. 
Interactor Arabidopsis

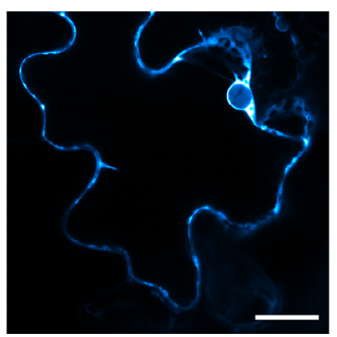

USPA; cytoplasm

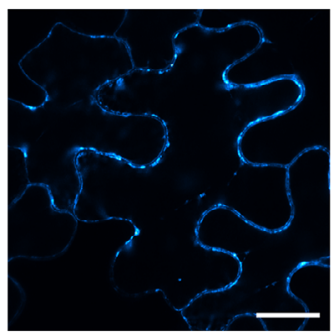

PRA1.F3; PS, ER

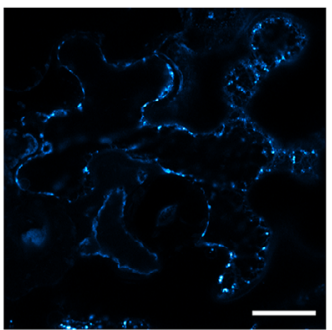

At3g13800; ER, PS

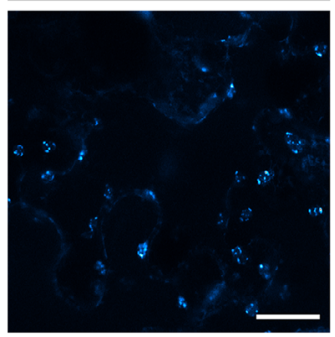

AHAS; chloroplasts, PS
Spinach ortholog

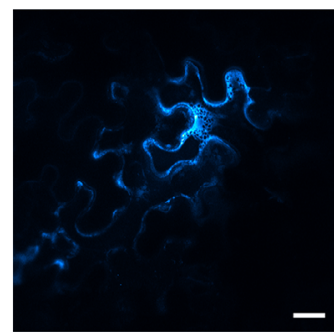

Spo22595; cytoplasm

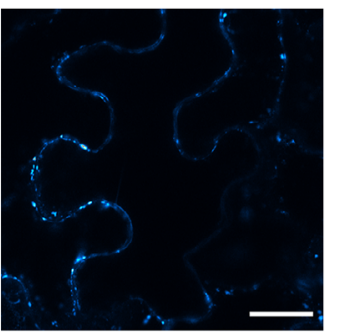

\section{Spo20405; PS}

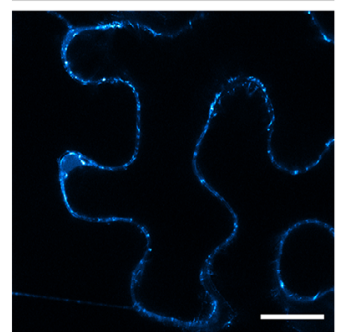

Spo20077; PS, ER, cyto.

Figure S4 (continued). Subcellular localization of Arabidopsis interactors and their spinach orthologs in $\mathbf{N}$. benthamiana.

Plant interactors fused to CFP (N-terminus) were produced in N. benthamiana. AHAS and ortholog Sp020077 were fused at its C- terminus to CFP. Images were taken $\sim 48$ to 72 hours after infiltration. Scale bars $=20 \mu \mathrm{m}$. 
Interactor Arabidopsis

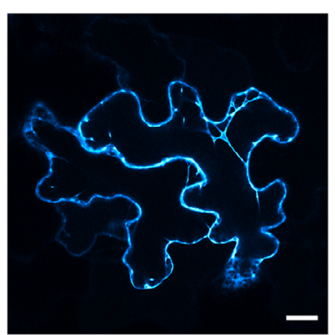

LSU1; PS, ER, cyto.

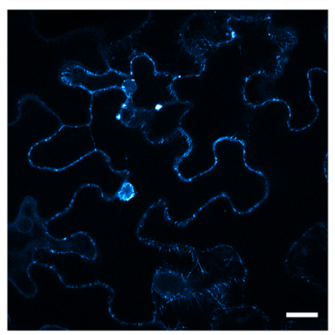

NAGK; PS, ER, nucl.

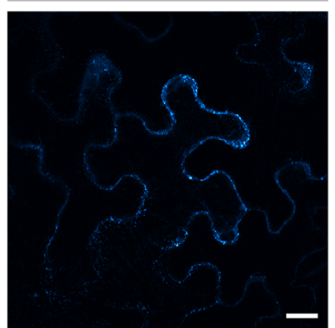

FBN1a; PS, nucleo-cyto.

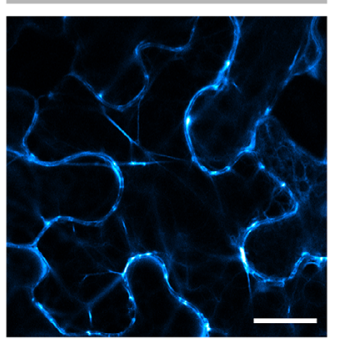

At5g56100; ER, PS
Spinach ortholog

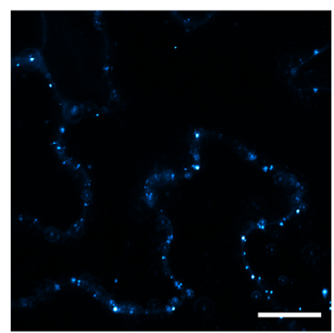

Sp022402; PS, cyto.

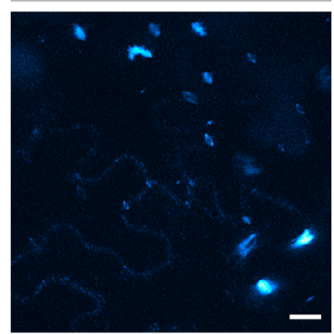

Spo15075; unclear

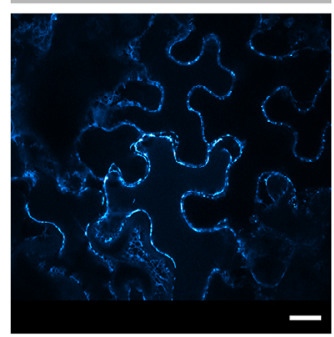

Spo20112; PS, ER, nucl.

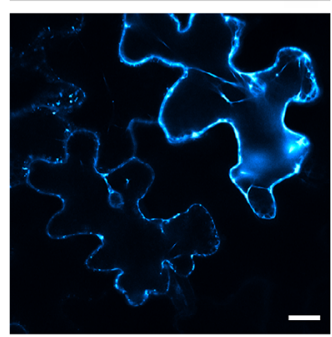

Sp012707; PS, ER, cyto.

Figure S4 (continued). Subcellular localization of Arabidopsis interactors and their spinach orthologs in $N$. benthamiana.

Plant interactors fused to CFP (N-terminus) were produced in N. benthamiana. AHAS and ortholog Sp020077 were fused at its C- terminus to CFP. Images were taken $\sim 48$ to 72 hours after infiltration. Scale bars $=20 \mu \mathrm{m}$. 


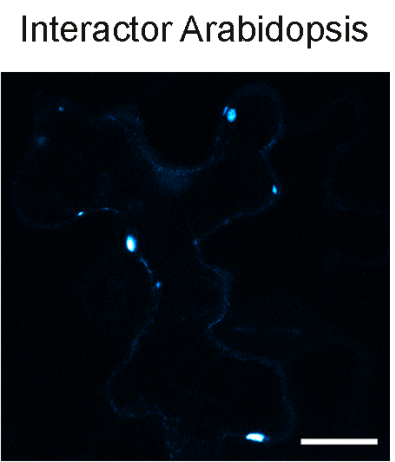

NAC078; unclear

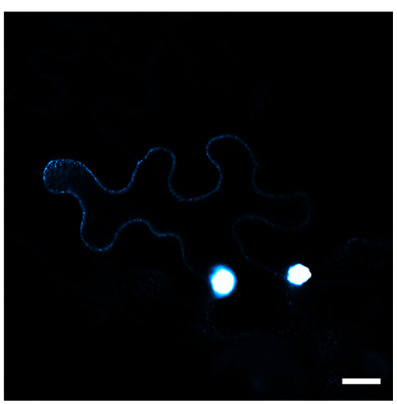

RAF2; nucleus
Spinach ortholog

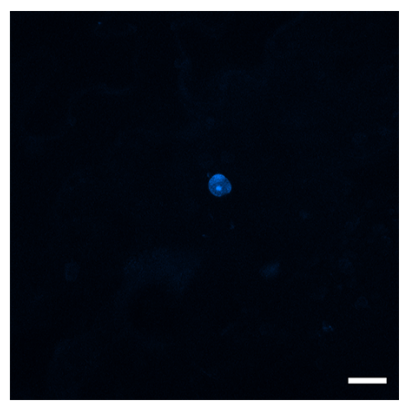

Spo16524; unclear

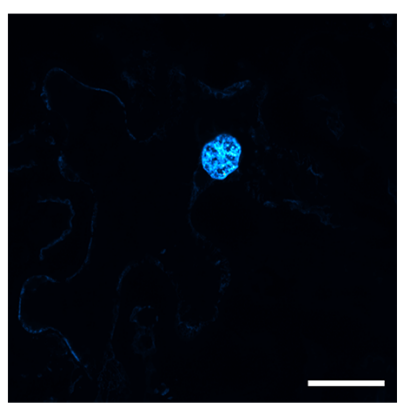

Spo14154; nucleus, cyt

Figure S4 (continued). Subcellular localization of Arabidopsis interactors and their spinach orthologs in

N. benthamiana.

Plant interactors fused to CFP (N-terminus) were produced in N. benthamiana. AHAS and ortholog Sp020077 were fused at its C- terminus to CFP. Images were taken $\sim 48$ to 72 hours after infiltration. Scale bars $=20 \mu \mathrm{m}$. 

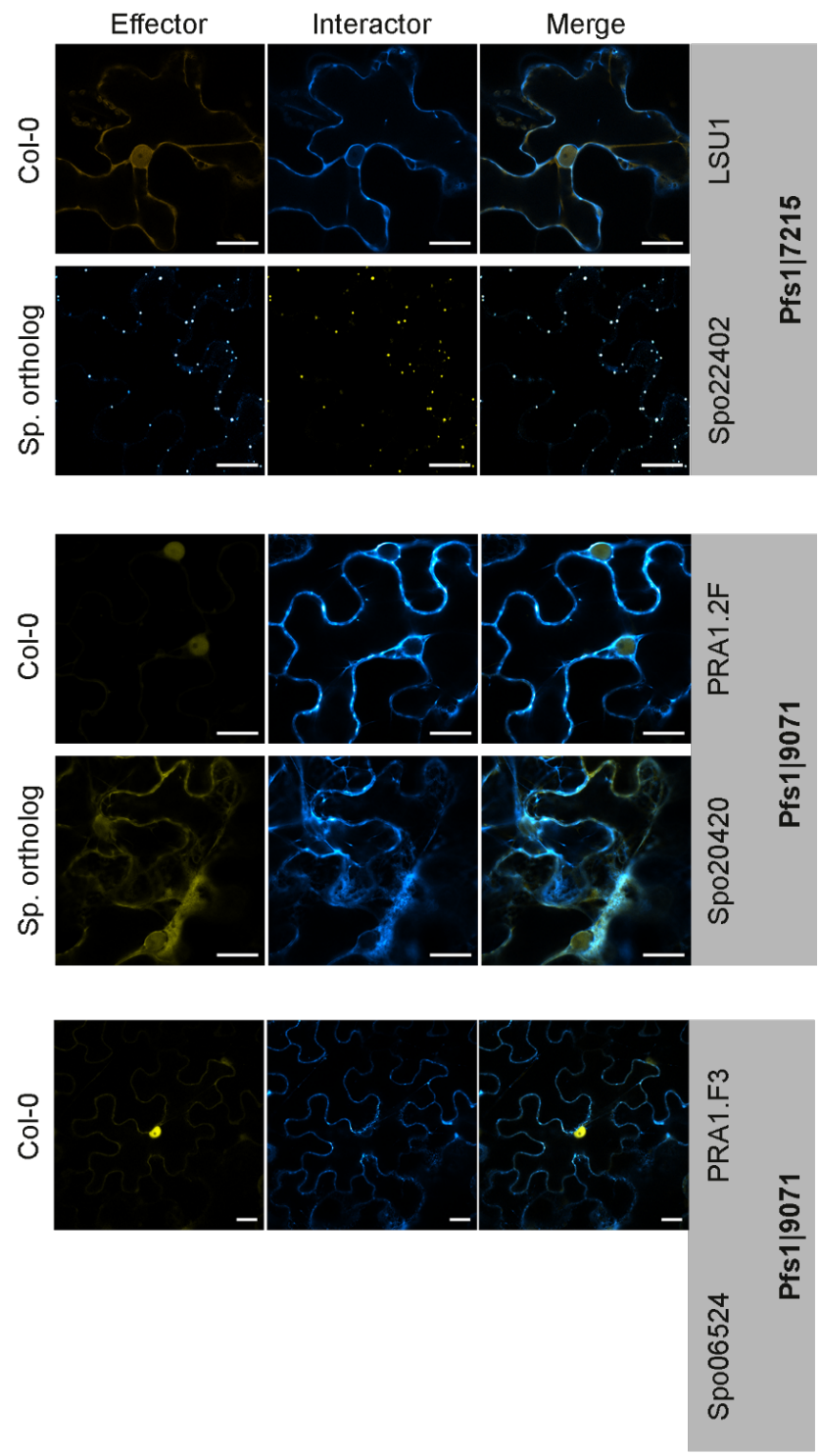

Figure S5. Co-localization of 5 Arabidopsis interactors and their spinach orthologs with candidate effectors of Pfs.

Plant interactors fused to CFP and candidate effectors fused to YFP (N-terminus) were transiently expressed in $N$. benthamiana. Co-localization was observed for all interactors except PRA1.F2 and F3. Subcellular localization of neither the interactors nor the effector candidate changed upon co-expression. Images were taken $\sim 48$ hours after infiltration and are representative of $>5$ cells. Scale bars $=20 \mu \mathrm{m}$. 

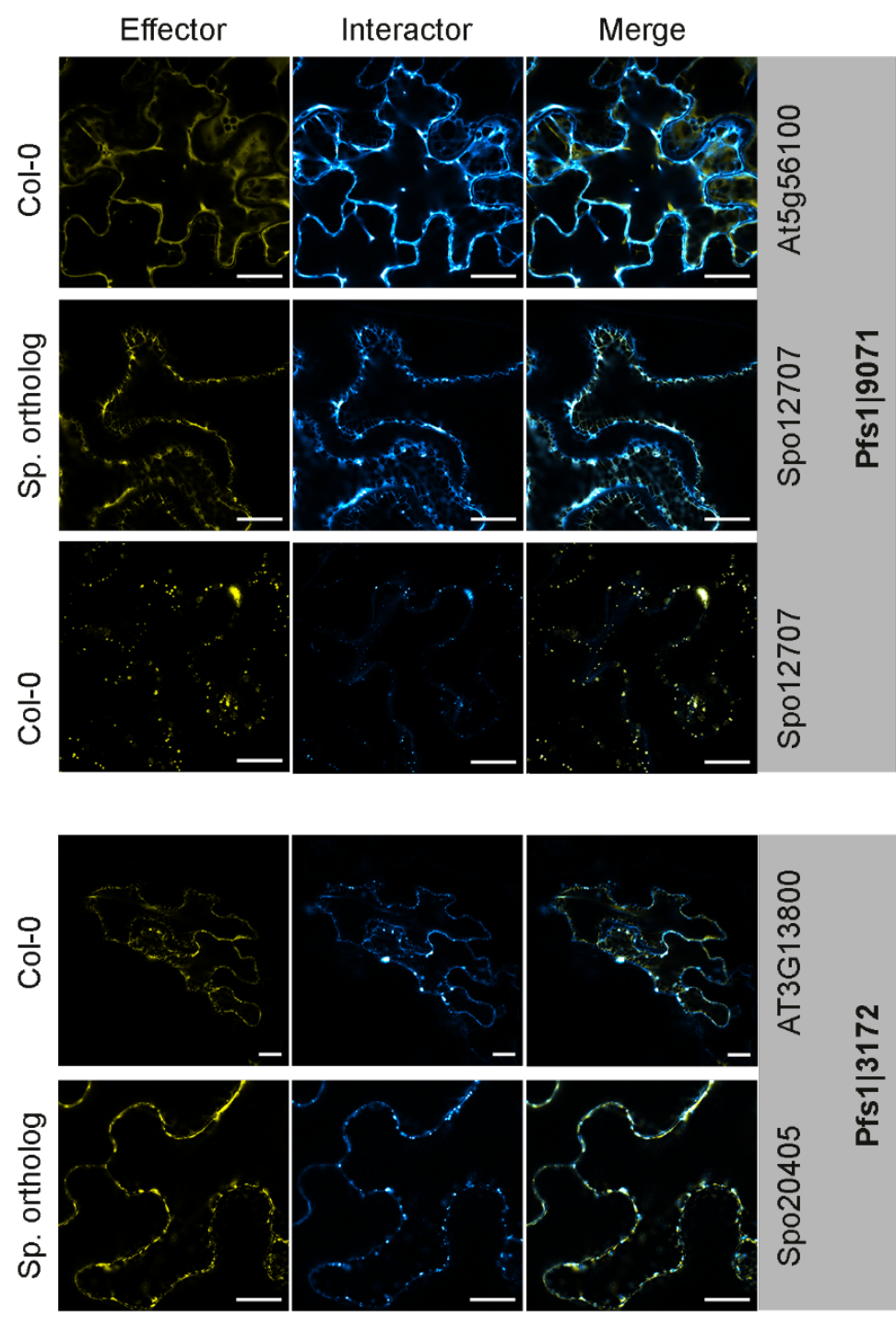

Figure S5 continued. Co-localization of 5 Arabidopsis interactors and their spinach orthologs with candidate effectors of $P f s$.

Plant interactors fused to CFP and candidate effectors fused to YFP (N-terminus) were transiently expressed in N. benthamiana. Co-localization was observed for all interactors except PRA1.F2 and F3. Subcellular localization of neither the interactors nor the effector candidate changed upon co-expression. Images were taken $\sim 48$ hours after infiltration and are representative of $>5$ cells. Scale bars $=20 \mu \mathrm{m}$. 


$$
2
$$




\section{$\underline{5}$}

General

discussion

Manon Neilen ${ }^{1}$, Guido Van den Ackerveken ${ }^{1}$

${ }^{1}$ Plant-Microbe Interactions, Department of Biology, Utrecht University, Padualaan 8, 3584 CH Utrecht, the Netherlands 


\section{General discussion}

The molecular interaction between phytopathogenic oomycetes and plants is a well-orchestrated and highly dynamic process [287, 288, 289]. As a basis for studying the molecular interactions between Peronospora effusa (Pfs) and spinach we sequenced reference race 1 of the spinach downy mildew (Chapter 2). During infection oomycetes secrete large numbers of effector proteins that help suppress the plant immune response and establish and maintain the infection [40]. Two types of hosttranslocated effectors are known in downy mildew species; the RxLR and CRN effectors [56, 120, 121]. In my thesis I describe a comparative analysis that revealed the conservation of these host-translocated effectors between oomycete species. In addition, plant proteins interacting with Pfs effectors were identified and characterized to get insights into possible molecular mechanisms enabling Pfs infection. Here I discuss the underlying genomic evolution of pathogenicity in oomycetes and put our findings on RxLR and CRN effectors in a broader context of susceptibility of spinach to Pfs.

\section{Evolutionary genomics of oomycete phytopathogens \\ Evolution of pathogenicity}

Intriguingly, the ability of eukaryotic microbes to cause disease (on plants) evolved independently in fungal and oomycete species [290] Additionally, species with intermediate lifestyles, like the hemi-biotrophic Phytophthora

5 species, are observed. Phytophthora infection has an initial biotrophic phase that is succeeded by a necrotrophic one [291].

It is generally thought that early saprotrophic species (that feed on already death plant tissue) have evolved to live off living plant tissue [290]DBoth saprotrophic and pathogenic fungi and oomycetes have filamentous hyphae and soilborne and waterborne spores, traits that may form the basis for the evolution of pathogenicity in both kingdoms [292]. Pathogenic species of both kingdoms have developed specialized feeding hyphae (haustoria) to accommodate their interaction with plants. 
Now, the growing number of available genomes of phytopathogens allows studying genomic signatures of pathogen lifestyles. Both the necrotrophic and biotrophic lifestyles require specialized genes and well-orchestrated gene expression to enable the successful infection of plants [293].

Comparing genomes of phytopathogenic species to that of saprotrophic ones provides insight into the molecular differences that underly the pathogenic lifestyle. Proteomes of phytopathogenic oomycetes and fungi were found to contain more hydrolytic enzymes, proteins with domains involved in carbohydrate metabolism, nutrient uptake, signaling and suppression of the host immunity response compared to nonpathogenic eukaryotic species [53, 294]. Many of the enriched domains were found in secreted proteins that are anticipated to be directly involved in virulence [53]. Among them are the host-translocated RxLR and CRN effectors in oomycetes that are discussed in the 'effector biology' part of this discussion.

Functional differences between proteomes of necrotrophic and (obligate/hemi) biotrophic species are also observed. They include enrichment of transporter functions in necrotrophs, enzymes involved in amino acid metabolism in hemi-biotrophs and nucleic acid metabolism and signal transduction (GTPase, lysophospholipase and tyrosine kinase activity) in obligate biotrophic species [294]. Also, obligate biotrophs were shown to have lost genes involved in metabolic pathways for the production of nutrients that can be obtained from the host. For examples, genes encoding sulfite, nitrite and nitrate reductases, a nitrate transporter and genes required for synthesis of arachidonic acid and polyamine oxidase were not found in the Hyaloperonospora arabidopsidis genome [59]. The nitrate reductases are also missing from the genomes of $P f s$ and other downy mildew species [29, 137, 187, 290]. The loss of genes encoding enzymes involved in processing and uptake of inorganic nitrates is also observed in Albugo laibachii and several fungal species (Melampsora larici-populina, Blumeria graminis, Puccinia. f. sp. graminis ) [290, 295]. It is the only example of a pathway that is lost in a broad range of pathogens, as the majority of the metabolic pathways are maintained in 
pathogen genomes [290]. These losses are considered a consequence of the obligate biotrophic lifestyle rather than a causal factor [197].

In chapter 2 we compared the secretomes of oomycete phytopathogens with different lifestyles. We found that evolutionary distinct biotrophic species, i.e. Albugo, Peronospora and Plasmopara showed similar sets of secreted proteins in terms of presence of Pfam domains. Secretomes of obligate biotrophs had fewer Nep-1 like proteins, proteins with RxLR domains (PF16810) and proteins with Sel1 repeat domains (PF08238) compared to hemi-biotrophic Phytophthora species. The reduction or loss of especially cytotoxic NLPs may aid the obligate biotrophic life, as they cause cytolysis and cell death [177]. Reduced numbers of proteins with PAN/Apple (PF14295), subtilase (PF00082) and Thrombospondin type 1 (PF00090) domains were observed in the secretomes of the obligate biotrophs compared to Pythium species. The PAN/Apple and subtilase domains are implicated in evasion of recognition and degradation of plant antimicrobial compounds respectively [179, 180]. PF00090-containing proteins have no known function and are abundant in the secretome of Pythium and Phytophthora species but absent the biotrophic species except for Plasmopara viticola.

\section{Genome size}

An interesting observation is that many filamentous pathogens have larger genomes than their non-pathogenic relatives [190, 296]. This observation contrasts the general idea of parasites and symbionts that evolve compact minimal genomes that lack unnecessary genes and entire metabolic pathways because metabolites can directly be obtained from the host [297, 298]. Among the largest phytopathogen genomes sequenced are those of the oomycete $P$. infestans $(240 \mathrm{Mb})$ and powdery mildew fungus Blumeria graminis (174 Mb) [58, 299]. Genome expansion is largely attributed to repetitive DNA, as a result of the amplification of transposable elements [58, 295, 296]. Transposable elements are mobile stretches of repetitive DNA that can 'travel' and amplify within genomes thereby altering DNA content and gene activity. Transposable 
elements increase genome size and induce genomic variation that may be advantageous for the adaptation of species [190]. The repetitive elements are often unevenly distributed across genomes resulting in distinct gene-dense, repeat-poor and gene-sparse repeat-rich genome areas [172, 300].

The gene-sparse, repeat-rich regions were found to evolve more rapidly than the rest of the genome resulting in an evolutionary pattern termed the 'two-speed genome' [190, 300]. These dynamic regions serve as a cradle for adaptive evolution while the rest of the genome contains more conserved genes that function in basal growth and developmental processes. Host-translocated RxLR and CRN effector genes often reside in the repeat rich areas of the genome resulting in their large expansion and rapid evolution [58]. The Pfs host-translocated effectors are modestly enriched in the gene sparse regions of the genome of Pfs 1 but the gene density difference between the effectors and core genes is not as strong as in the $P$. infestans genome (Chapter 2).

Pathogens with flexible genomes can more easily cope with resistant hosts by losing or altering genes and can even adapt to infect a new host plant species. Assuming that it is only a matter of time before a host plant species evolves resistance to a pathogen, pathogens with flexible genomes are able to more frequently jump to a susceptible or new host and are thus more likely to persist over evolutionary time. This flexibility is proposed to outweigh the disadvantages of a large and costly genome [190].

Genome flexibility is argued to be beneficial especially for biotrophic pathogenic species as they depend on their host and have to avoid recognition by the ever adapting host immune system [290] However, genome sequencing revealed an opposing trend in the genome sizes of downy mildews of the Peronospora genus and other obligate biotrophic oomycete and fungal species (Chapter 2, [60, 137, 187, 301, 302]). 
The Peronospora species sequenced so far, including Pfs, have small genomes, ranging between from $\sim 30$ to $60 \mathrm{Mb}[60,137]$. We found that the assembled Pfs 1 reference genome size is $32.4 \mathrm{Mb}$ (the estimated size based on $k$-mer analysis $36.2 \mathrm{Mb}$ ), which is comparable to the Pfs genome size reported by Fletcher et al ( $\sim 39 \mathrm{Mb})$, based on measured nuclear DNA using flow cytometry [187]. We estimated the repeat content of Pfs 1 to be $\sim 27 \%$ (8.8Mb) (Chapter 2) which is low compared to that of for instance $P$. infestans (74\%), similarly to the repeat content of other Peronospora species [58]. The relatively low content of repetitive DNA may be underestimated as the genome is quite fragmented and repetitive DNA is particularly hard to assemble. Given the proposed advantages of large flexible genomes, this trend in downy mildews is remarkable. Yet, Peronospora is a successful genus with many species that are virulent on a wide range of plant species [17].

Alternatively to genome variation by repetitive DNA and transposable elements, sexual reproduction could increase variation in the genomes of downy mildews [290]Genetic recombination in smut fungi is facilitated strictly through sexual reproduction, which may explain why their genomes are small yet flexible enough to adapt. Although oomycetes can reproduce sexually they are thought to primarily propagate through asexual reproduction, suggesting that the contribution of sexual reproduction to genetic variation is smaller compared to that in smut fungi [8]. It is not clear how sufficient genome flexibility is maintained in these relatively small downy mildew genomes. Possibly, the small genomes reflect reduction of unnecessary genes while maintaining enough flexibility required for survival. We observed that a smaller proportion ( $4-7 \%)$ of the proteome of Hyaloperonospora/Peronospora is secreted compared to Phytophthora species ( 7-10\%) (chapter 2). The secretomes of these oomycete species are functionally comparable in terms of carbohydrate active enzymes but the numbers and thus diversity of secreted proteins is reduced in downy mildew species. This reduction may help to avoid recognition by the plant immune system and contribute to the reduced genome size. 


\section{Genomics and taxonomy}

Systematics is perhaps best described as the science that satisfies the human desire to order its natural surroundings into conceivable, orderly units. The idea that similar species descent from a common ancestor, first proposed by Charles Darwin, gave way to phylogenetic inference of the 'tree of life'. Systematics classifies species into monophyletic groups, i.e. groups that are derived from a common ancestor (first proposed by Henning) and names them accordingly (taxonomy) [303]. In taxonomy species are organized into ranks; genera, then families, orders, classes and so on in order of inclusiveness. Traditionally, taxonomy is based on morphological and biological traits [304]. The emergence of molecular genetics provides an additional, powerful trait to help solve evolutionary relationships. In many cases, molecular genetic data showed that traditionally recognized clades are not monophyletic. In those cases, morphological and biological similarity may result from convergent evolution or genetic drift rather than ancestry, thereby complicating the definition of families, genera and even species.

Within the oomycetes these discrepancies in taxonomy are especially evident in the large and diverse taxa of Pythiaceae and Peronosporales. While some authors divide the crown oomycete species into three orders; Pythiales, Peronosporales and Albuginales [4, 292], others prefer to place the phytopathogenic Pythium species in a family (Pythiaceae) within the Peronosporales order [6, 20]. This lack of consensus is nicely illustrated by the number of hits obtained from Google Scholar searches for the different orders and families and combinations of those (Table 1). The combination 'Pythiaceae -Peronosporales' (4.540) is used slightly more often in scientific literature than 'Pythiales, Peronsporales' (3.790). Remarkably, 4.200 hits are obtained for the combination 'Pythiales, Pythiaceae'; a Pythiaceae family within a Pythiales order. 
Table 1. Usage of the taxonomic classifications 'Pythiaceae' or 'Pythiales' in scientific publications (based on Google Scholar).

\begin{tabular}{|l|l|}
\hline Query & Number of hits \\
\hline Pythiales & 5.870 \\
\hline Pythiaceae & 8.170 \\
\hline Peronosporales & 13.200 \\
\hline Peronosporales, Pythiales & 3.790 \\
\hline Peronosporales, Pythiaceae & 4.540 \\
\hline Pythiales, Pythiaceae & 4.200 \\
\hline
\end{tabular}

Number of Google Scholar hits for different queries, obtained in May 2020.

It is widely accepted that the Pythiales or Pythiaceae are not monophyletic but there is a lack of consensus on how to deal with the taxonomy of the many species [20,305]. Following Hennings idea of taxonomy informed on monophyletic groups, there is no basis for defining an order of Pythiales that includes all currently identified Pythium species. Multigene phylogenies show that Pythium species like Pythium ultimum and Pythium splendens are not monophyletic with the other Pythium species but are more closely related to Phytopthora species. For the same reason, a monophyletic Pythiaceae family (that encompasses all Pythium species) within the Peronosporales order is not supported [20]. Dividing the Pythium species into two families within the Peronosporales order or renaming Pythium clade I,F,G species to solve the polyphyly would provide an intuitive organization of Pythium species that will aid further communication about the crown oomycete taxa.

Similarly, the large group of Phytophthora species is now widely accepted to be paraphyletic, with the downy mildew species embedded among the different Phytophthora clades, asking for re-evaluation of their taxonomy [306] In multiple gene/protein and full genome comparisons in this (Chapter 2) and other studies [27, 173], downy mildew species were suggested not be monophyletic. The lineage of downy mildew species with pyriform haustoria (in the genera Plasmopara, Bremia) was found not to be monophyletic to the lineage that encompasses the graminicolous grass-infecting downy mildews, the downy mildews with colored conidia 
(like Peronospora) and the brassicolous downy mildews. Future studies including more species and using curated sets of conserved genes are needed to get an unambiguous answer to the Peronosporales phylogeny.

\section{Effector biology}

Genomes of phytopathogenic oomycetes encode large numbers of effector proteins. These proteins are secreted from the oomycete and can function either outside or inside the plant cell. Many effectors have evolved to manipulate plant processes to avoid or suppress plant immunity [80, 198], e.g the responses triggered by recognition of pathogen-associated molecular patterns (PTI) or effectors (ETI). Two types of host-translocated effectors are known from Peronsporales species, the RxLR and CRN effectors. For some of those effectors of Peronsporalean species other than Pfs the molecular role in virulence was studied in more detail (Chapter 1) but the function of the majority of RxLR and CRN proteins in still unknown.

The sequencing and assembly of the Pfs1 reference genome cleared the way for identifying its host-translocated effectors and studying their individual contribution to the interaction with the spinach host. We identified 99 candidate host-translocated RxLR and 14 candidate CRN effectors in the secretome of Pfs1 (Chapter 2). The work of Joël Klein, that is complementary to the work described in this thesis, described the search polymorphisms in effectors that are associated with the breaking of resistance in spinach [207]. Resistance to specific races of pathogens is generally mediated by intracellular R-proteins that recognize hosttranslocated effector proteins [287]. Intracellular nucleotide-binding leucine-rich repeat receptors (NB-LLR receptors) directly or indirectly recognize effectors [307]. The recognition triggers an immune response termed effector-triggered immunity (ETI) [80]. However, pathogens such as Pfs did not evolve effectors to be recognized, but rather to function in manipulation of host processes to allow successful infection. In this thesis I describe the study of the molecular interaction between individual RxLR and $\mathrm{CRN}$ effectors and plant proteins in a first step towards understanding 
how these effectors contribute to disease.

The combined works provide an integrated view of Pfs effectors related, on the one hand to breaking of resistance, and on the other hand to the molecular interactions with plant proteins.

\section{The search for resistance breaking effectors in the Pfs}

The recognition of an effector mediated by a plant R-gene confers a very strong immune response that induces resistance to the pathogen [308]. When recognition occurs, this overrules the virulence function of the effector and the activated plant immune system reduces the fitness of the pathogen. The detection of effectors by the plant leads to diversifying selection pressure so that recognized effectors may be lost or altered or new effectors suppressing the immune response evolve. Individual effectors are usually not crucial for survival and there is redundancy in effector repertoires [200, 309]. In Pfs, these dynamics lead to the continuous emergence of new races that break R-gene mediated resistance of newly bred spinach varieties [30, 32]. Within the work of Joël Klein the genomes of 16 races and 8 field isolates of Pfs have been sequenced [207].

Effector polymorphisms between the races were used to identify effectors that may be recognized by resistance loci using a differential set of spinach lines. Most strikingly, deletion of candidate effector Pfs1|1792 was found to be associated with the breaking of resistance conferred by RPF3 and polymorphisms in three other effectors were associated to breaking RPF5 (effector Pfs1|3839) and to RPF2 (effectors Pfs1|6786 and Pfs1|9985) resistance. Systematic screening of effector proteins can be employed to identify R-genes, in cultivated and wild plant species, by assessing their response to effectors and linking it to genomic loci in the plants [310]. This is especially instrumental in resistance breeding of crops.

The downside of R-gene mediated resistance is that though it is very effective, it is usually not durable [288]. Many resistances rely on 
the recognition of single effectors that are often not crucial for pathogen success [311]. Pathogens have large effector sets that have redundant functions, so upon recognition they can easily loose or alter effectors to avoid recognition.

\section{Towards the virulence function of Pfs effectors}

To better understand the role of individual host-translocated effectors I studied their possible virulence function by identifying their putative plant targets. In other words, how do effectors interfere with plant processes to make plants susceptible to Pfs? To this end, I first studied if effectors are present in related species to identify those that are conserved between species. Little conservation of effector proteins between related species is observed, potentially as a result of positive selection to avoid the recognition by the plant immune system [75]. Still, a number of RxLR effectors are conserved among phytopathogenic oomycetes, possibly because they contribute significantly to virulence [312]. Although conserved effectors may be the 'odd ones out' due to positive selection to avoid recognition, the fact that some are conserved makes them interesting targets. These effectors may target conserved interacting plant proteins that are involved in the infection process and contribute to plant disease susceptibility. The identification of Arabidopsis proteins that interact with Pfs effectors supports the idea of conserved plant targets across species (Chapter 3). These so called "Achilles heel" effectors can be used to identify and understand molecular manipulation of plants [308].

We identified the potential virulence targets of 18 candidate Pfs host-translocated effectors from a yeast two-hybrid library of Arabidopsis protein fragments (Chapter 3). The spinach ortholog of each Arabidopsis prey protein was identified within the proteome of spinach line Sp75 [241]. The interaction between the spinach orthologs and the Pfs hosttranslocated effectors will have to be confirmed in future $\mathrm{Y} 2 \mathrm{H}$ screens. For six plant preys and their spinach orthologs fused to fluorescent proteins we showed that they reside in the same subcellular compartment as the 
effector when co-expressed in N. benthamiana. For five of them we found that the presence of the candidate effector induced re-localization of the target to a different subcellular compartment (Chapter 4). The subcellular localization of two effectors, Pfs1|6786 and CRN Pfs1|9976, also changed upon co-expression with the plant interactors. Altered localization upon co-expression is suggestive of physical interaction between the proteins. A next step would be to confirm the interaction in planta by using coimmunoprecipitation (co-IP) or Fröster resonance energy transfer by fluorescence lifetime imaging (FRET-FLIM) [313].

Once the interaction is established the question that remains is what the effect is of binding of the plant protein by the candidate effector on processes in the plant and susceptibility to Pfs. Studies in chapter 4 provide a first idea on the processes affected. The effectorinteractor proteins roughly fall into 5 categories; immune signaling (hormonal, transcriptional, ROS) defense, post-transcriptional regulation, metabolism, and other. To our knowledge Pfs1|6786 and Pfs1|3869 are the first oomycete effectors that could be involved in the manipulation of amino acid metabolism.

The outcome of the interaction between effector and protein depends on the function of the plant protein and the effect of the interaction on that functionality. Effectors are commonly observed to target proteins to suppress immunity, thus enhancing plant susceptibility [198]. Additionally oomycete pathogens exploit plant proteins termed susceptibility genes (S-genes) to promote disease [90, 99, 314]. Host genes that contribute to susceptibility of plants to pathogens are termed susceptibility (S) genes [315]. Examples include Rho-like GTPases (OsRAC4 and OsRAC5) that are required for full susceptibility of rice to Magnaporte grisea [316] and Arabidopsis to powdery mildew (ROP6) [317], proteins involved in amino acid metabolism [318] SWEET transporters that transport glucose and sucrose across cell during infection [319, 320] and negative regulators of immunity [314]. S-genes are interesting targets for plant breeding because the loss or mutation will limit or abolish pathogen infection. It is expected that S-gene based resistance is more durable than R-gene 
based resistance because the pathogens has to overcome a dependency on a host factor rather than evading recognition by an R-gene [308]. For that, a pathogen has to acquire a new function which is more difficult than to lose one [296]. This is especially evident in biotrophic species that depend on host factors, such as particular metabolites that they cannot produce themselves [314, 315].

\section{Combining inter-and intra-specific comparative effector data}

The above-mentioned complementary approaches to asses intra-species variation to identify potential recognized effectors and inter-species conservation to identify Achilles heel effectors can be combined to build a further framework of effector conservation. Apart from the polymorphisms in different $P f s$ races, 41 candidate effectors were found to be conserved in all sequenced $P f s$ isolates [207]. That is, the protein sequence of those 41 effector proteins had no homozygous amino acid changes between Pfs isolates. When we overlay these data with the comparative study described in chapter 3, we observe that fifteen of these conserved effector proteins are unique to Pfs and are possibly important in the compatible interaction between Pfs and spinach. The other proteins were conserved in orthogroups that have proteins of Pfs and other downy mildews (6), other Peronosporales (4) or proteins from more distantly related species used in the comparison (16) (Albugo, Pythium, Peronosporales). Their conservation within Pfs races points to an important virulence function within Pfs while effector conservation between species points to targeting of conserved plant proteins in multiple plant species. Studying plant proteins targeted by conserved effectors may reveal molecular mechanisms employed in a broader range of plant species. Table 2 provides a shortlist of interesting candidate effectors, conserved within Pfs and between Pfs and other species for future study on their role in the infection process. Their putative translocation domains, WY-folds, transmembrane helices and conservation with proteins of other species and identified interactors as described in chapter $\mathbf{3}$ are indicated. 


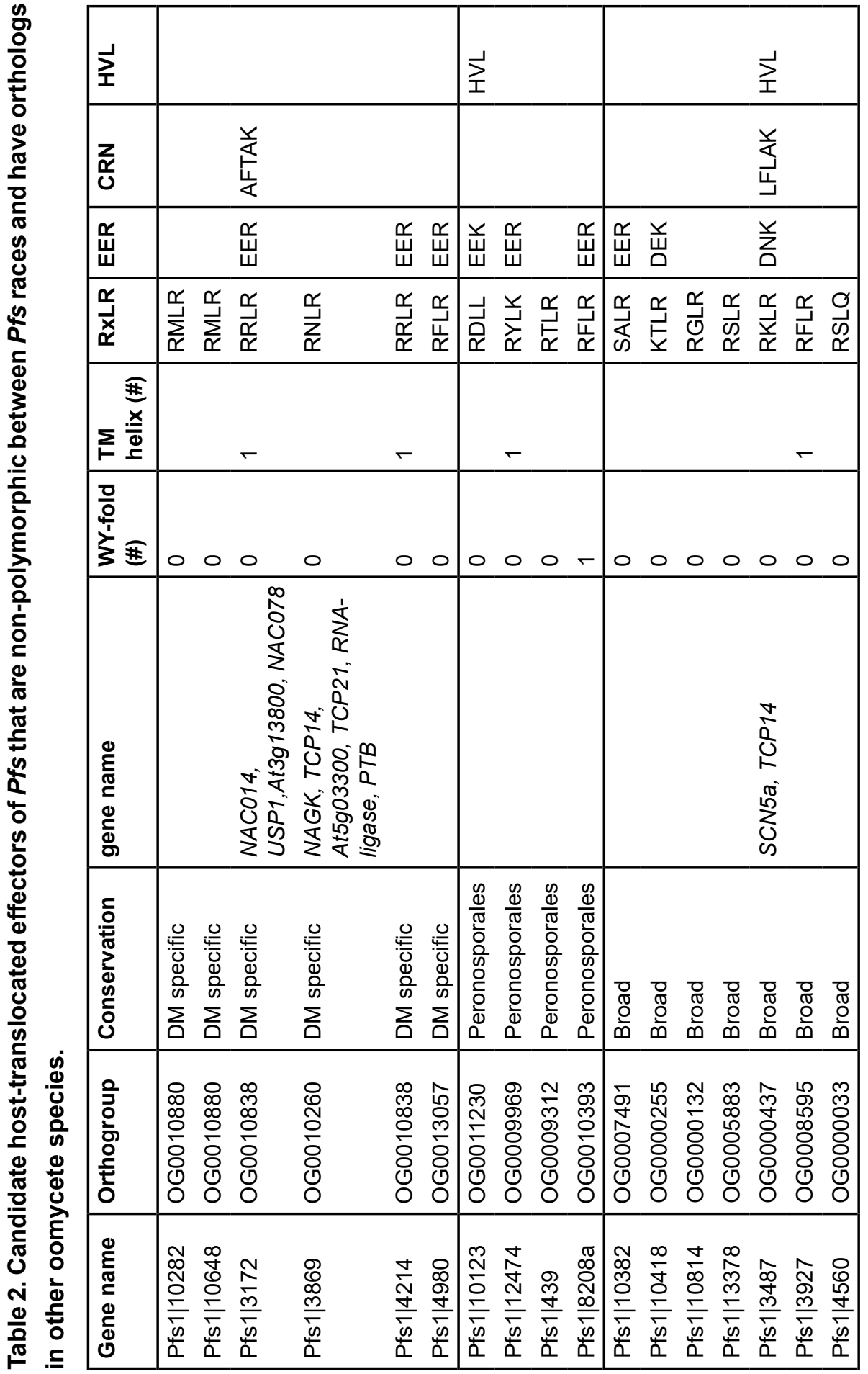




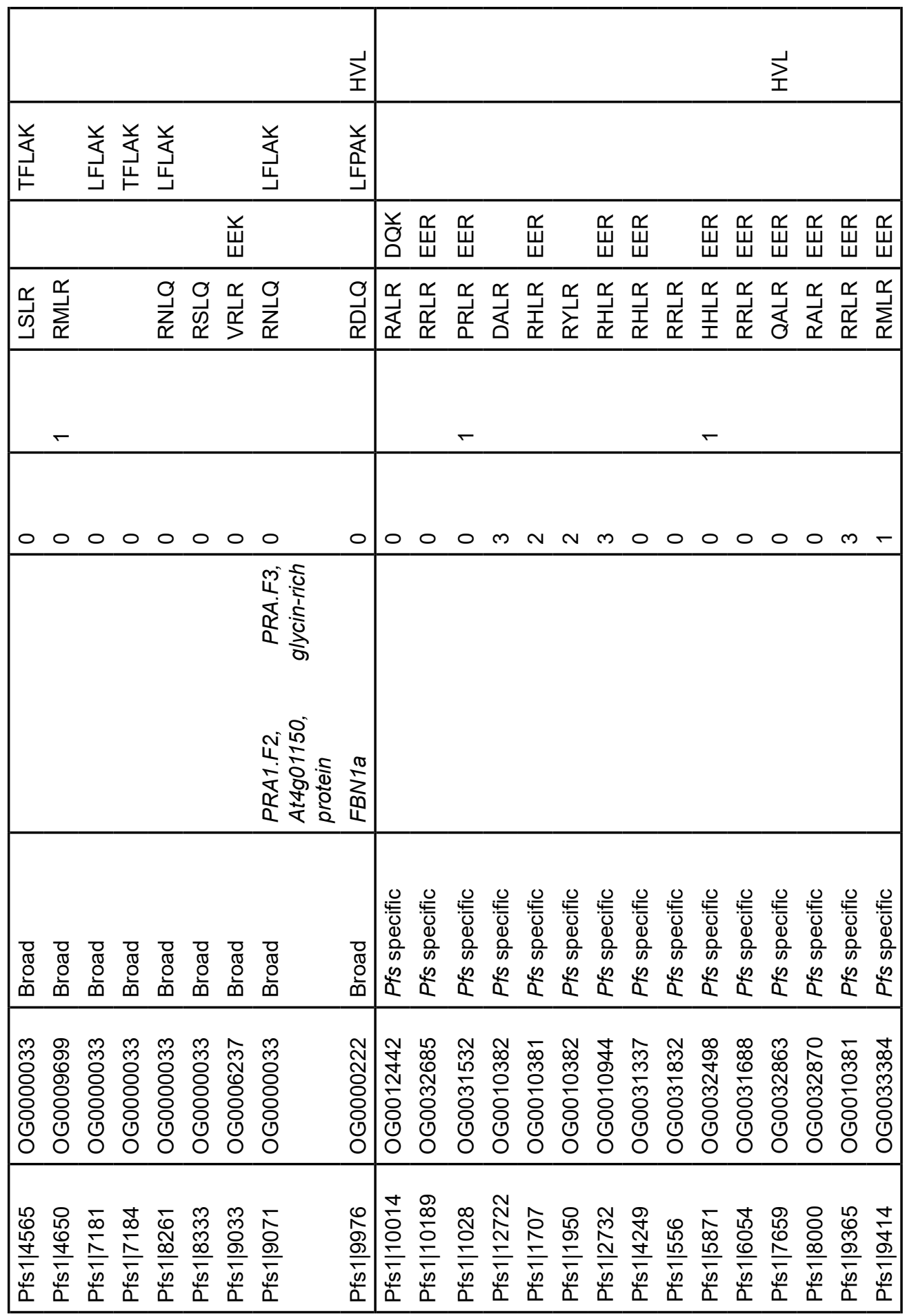


Five (Pfs1|3172, Pfs1|3869, Pfs1|3487, Pfs1|9071 and Pfs1|9976) of the eight candidate effectors studied in this thesis are included in the list of conserved effectors in table 2 (Chapter 3 and 4). For example, Pfs1|3172 was found to interact with two membrane-anchored NAC transcription factors, AtNAC078 and AtNAC014. Pfs $1 \mid 3172$ is conserved between $H$. arabidopsidis, $P$. belbahrii and Pfs. The proteins have a conserved RxLR-EER motif and a C-terminal transmembrane domain. It is likely that these downy mildew proteins have similar functions in their respective host plants.

An interesting candidate identified using this shortlist is candidate RxLR effector Pfs1|439. It is conserved between $P$. parasitica, P. capsici, P. sojae, P. belbahrii, Plasmopara halstedii and Plasmopara viticola (Fig. 1). All proteins except Phal02046 are predicted to be secreted and the $\mathrm{RxLR}$ domain, located in the $\mathrm{N}$-terminus, is strongly conserved. The C-terminal effector domain is more diversified although some residues are conserved, which is a common pattern for effector proteins [321]. The intra and inter species conservation of Pfs1|439 makes the effector an interesting candidate for further study of its virulence function. Studying the interaction between these conserved effectors and their interactors ay aid the identification of conserved S-genes that may be employed for resistance breeding. 


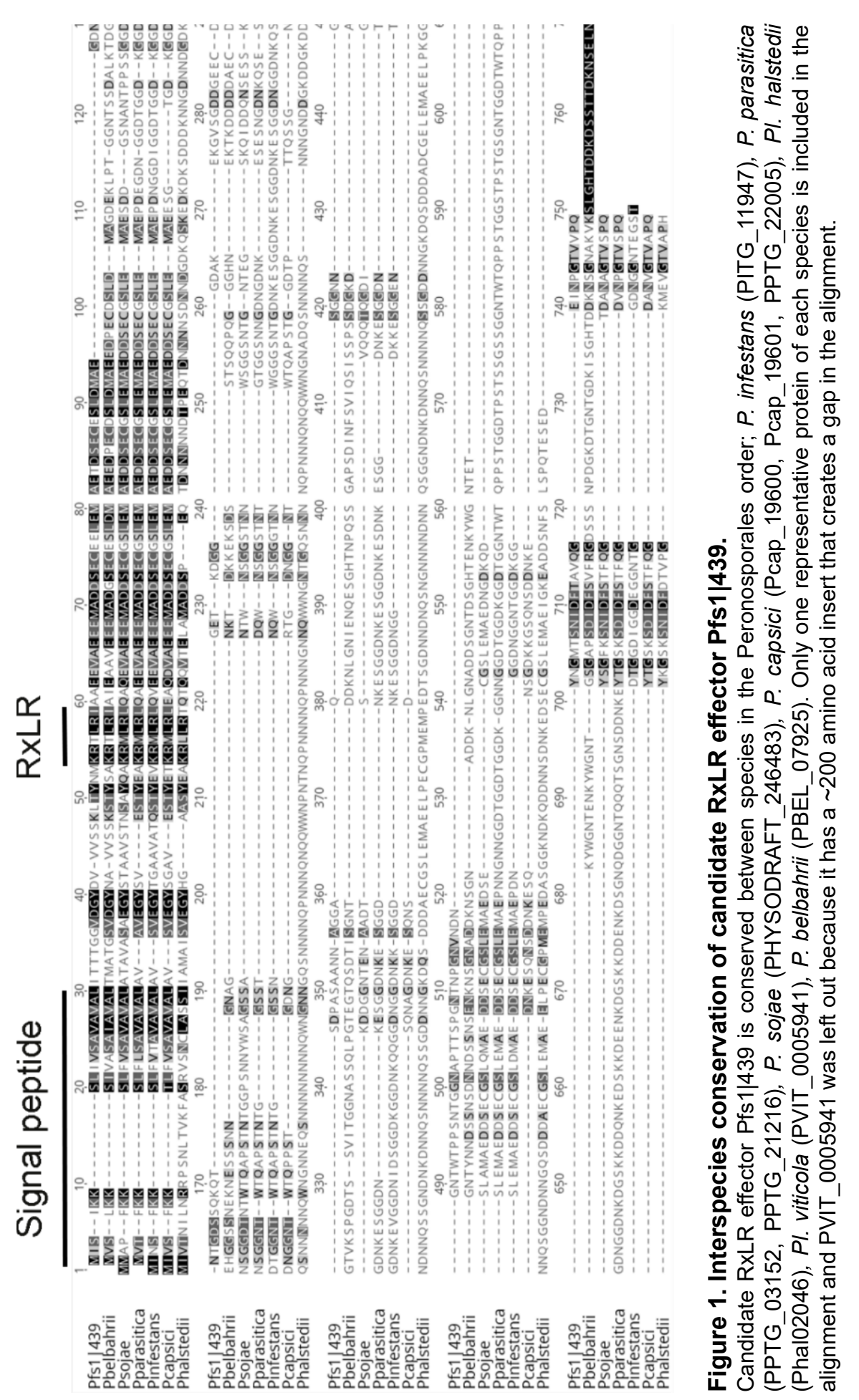




\section{Outlook}

The sequencing and assembly of the reference genome of Pfs1 gave way to the identification and characterization of RxLR and CRN effectors. In this thesis I describe the identification of the potential virulence targets of the first 27 candidate host-translocated effector proteins. Eight candidate effectors and fourteen interacting plant proteins were further characterized to provide a first insight into the possible role of individual effectors during Pfs-infection. This study confirmed co-localization of the majority of the studied plant proteins with the candidate effectors within Benthamiana nicotiana cells. In some cases, co-expression of the interactor and effector induced subcellular relocalization of either protein, an additional indication of a physical interaction between the proteins. After confirmation of the interaction by co-IP or FRET-FLIM the question that remains is how the interaction with the effector alters the function of the plant protein during infection. A quick way to mimic the inhibition or stabilization of a plant protein by its interaction with the effector is to study plants in which the interactor proteins are mutated, silenced or overexpressed. Disease assays on Arabidopsis T-DNA insertion lines, mutated in genes encoding interactors identified in chapter 3 and further studied in chapter $\mathbf{4}$, using disease assays with $H$. arabidopsidis could quickly reveal differences in susceptibility to downy mildew infection. The advantage of using $H$. arabidopsidis is that the T-DNA insertion lines are available, and the finding of altered susceptibility would suggest a role of the plant protein in susceptibility that is not restricted to the Pfs - spinach

5 interaction. Ideally, the assays should be performed in spinach because the effector interactors may be specific for Pfs effectors and hence may not have an effect on the susceptibility of Arabidopsis to $H$. arabidopsidis. Silencing of the interactor genes in spinach could be achieved by RNAi mutagenesis.

Developing reliable and high-throughput effector screening assays for application in spinach is of crucial important to improve the understanding of $P f s$ effector functions. Especially the delivery of proteins into spinach tissue should be optimized. We found that expression of 
fluorescent effector-fusions in spinach leaves yielded few transformed cells and lower levels of expression compared to fusion proteins expressed in $N$. benthamiana using the same vector (CaMV 35S promotor) (Chapter 4). Perhaps the expression levels could be improved by using a different promotor.

To improve effector delivery, other systems of protein delivery systems could be explored. Previously the expression of Hpa RxLR29 fused to a type III secretion signal in Pseudomonas syringae pv. tomato (Pst) was shown to suppress activation of the plant immune response, indicating the potential for the use of bacteria for delivery of oomycete effectors in planta [321]. Plant transformation methods independent of Agrobacterium T-DNA transfer, like particle bombardment, may also be tested and optimized for spinach plants [322]. (Stable) transformation or genome editing of spinach plants would aid the functional analysis of interactor genes in disease susceptibility in future experiments.

Finally, research on obligate biotrophic pathogens like Pfs would greatly benefit from the ability to manipulate and transform the pathogen. Systematic introduction or knock-out of host-translocated effectors in different $P f s$ races would greatly enhance the understanding of the contribution of individual effectors to virulence. Transformation of Phytophthora protoplasts or zoospores with plasmids containing effectors fused to fluorescent proteins is now quite well established, although not easy [323, 324]. Additional challenges include wrong localization of proteins, formation of insoluble aggregates and instability of transgene expression [325]. Transformation protocols have been adjusted for specific species and sometimes races to improve expression, hopefully this will be accomplished for obligate biotrophic oomycete species in the near future as well. 
Revealing the function of conserved effectors will greatly aid resistance breeding in crops. Most forms of resistance used in breeding, so far, are based on recognition of effectors by R-loci. Due to redundancy in the effector repertoire, oomycetes can easily loose effectors that are recognized, hence avoiding their recognition. However, conserved effectors may not be dispensable as they form the Achilles heel of the pathogen, so R-genes against those effectors may be more durable.

Additionally, employing natural or induced variation in the plant proteins that interact with conserved effectors to avoid interaction possibly results in more durable resistance of the plant. When the interaction between effector and plant protein is abolished the effector will no longer be able to manipulate the underlying plant process resulting in reduced fitness of the pathogen or elimination of the infection all together. If the interacting plant protein is a conserved effector target, variation in its sequence may be applicable to a wider range of crop species and pathogens [308]. Taken together, the study of conserved effectors and their interacting plant proteins provides a great opportunity to understand the molecular interaction between plants and pathogens and translate that knowledge to resistance breeding. 


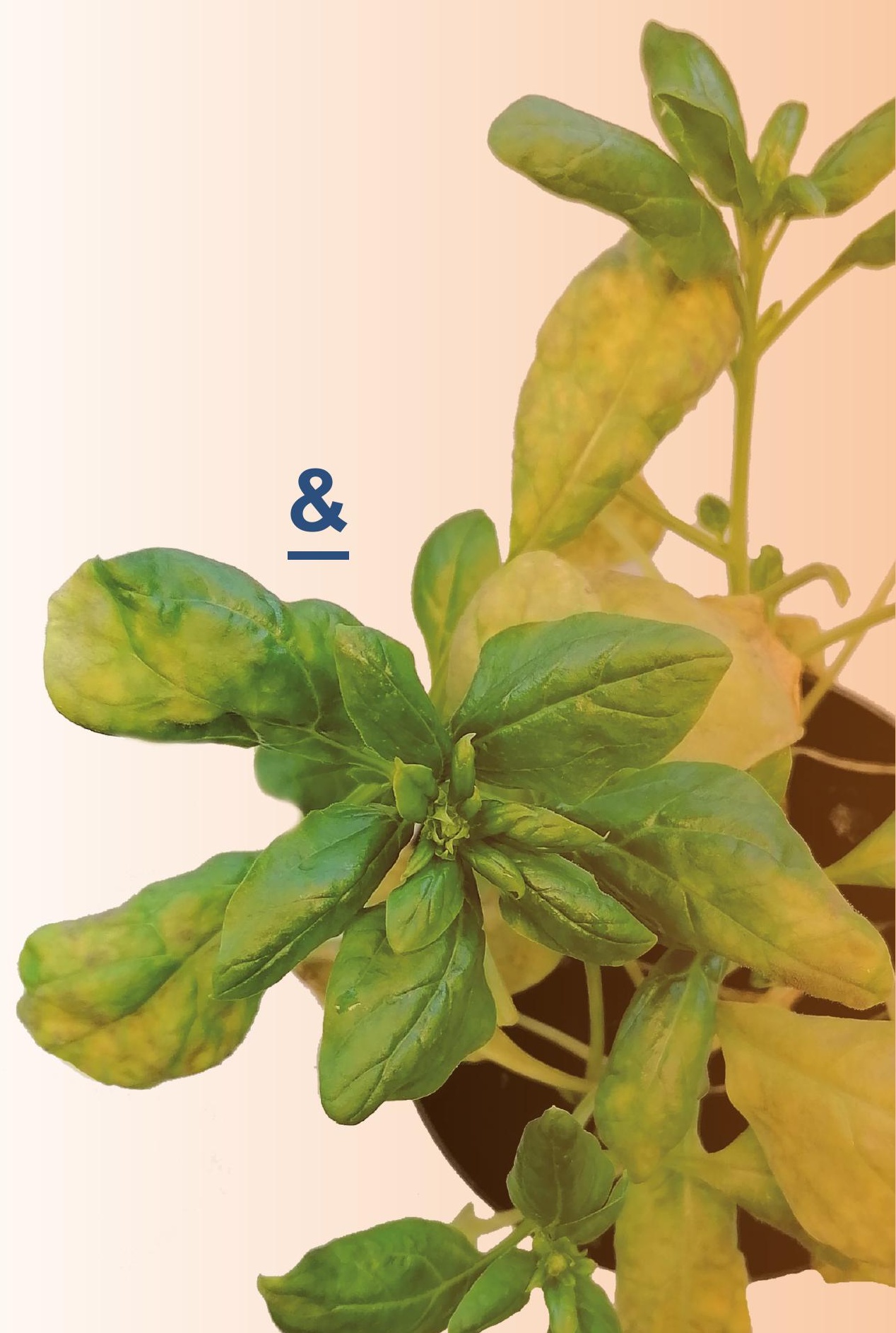


References

Summary

Samenvatting

\section{Acknowledgement I \\ Dankwoord}

About the author

List of publications 


\section{References}

1. Choi Y-J, Thines M. (2014). (2288) Proposal to reject the name Botrytis farinosa (Peronospora farinosa)(Peronosporaceae: Oomycetes). Taxon; 63:675-676.

2. Lee SC, Ristaino JB, Heitman J. (2012). Parallels in intercellular communication in oomycete and fungal pathogens of plants and humans. PLOS Pathog; 8:e1003028.

3. Baldauf S. (2003). The deep roots of eukaryotes. Science; 300:1703-1706.

4. McGowan J, Fitzpatrick DA. (2020). Recent advances in oomycete genomics. Adv Genet; 105:175-228.

5. Baldauf SL, Roger AJ, Wenk-Siefert I, et al. (2000). A kingdom-level phylogeny of eukaryotes based on combined protein data. Science; 290:972-7.

6. Thines M. (2014). Phylogeny and evolution of plant pathogenic oomycetes - a global overview. European Journal of Plant Pathology; 138:431-447.

7. Thines M, Kamoun S. (2010). Oomycete-plant coevolution: recent advances and future prospects. Curr Opin Plant Biol; 13:427-33.

8. Kamoun S, Furzer O, Jones JD, et al. (2015). The Top 10 oomycete pathogens in molecular plant pathology. Mol Plant Pathol; 16:413-34.

9. Diéguez-Uribeondo J, García MA, Cerenius L, et al. (2009). Phylogenetic relationships among plant and animal parasites, and saprotrophs in Aphanomyces (Oomycetes). Fungal Genetics and Biology; 46:365-376.

10. Gaulin E, Jacquet C, Bottin A, Dumas B. (2007). Root rot disease of legumes caused by Aphanomyces euteiches. Molecular Plant Pathology; 8:539-548.

11. Holub E, Brose E, Tör M, et al. (1995). Phenotypic and genotypic variation in the interaction between Arabidopsis thaliana and Albugo candida. Molecular plant-microbe interactions: MPMI; 8:916-928.

12. Heller A, Thines M. (2009). Evidence for the importance of enzymatic digestion of epidermal walls during subepidermal sporulation and pustule opening in white blister rusts (Albuginaceae). Mycological research; 113:657-667.

13. Pétrie GA. (1975). Prevalence of oospores of Albugo cruciferarum in Brassica seed samples from western Canada, 1967-73. Canadian Plant Disease Survey; 55:19-24.

14. Thines M, Choi Y-J, Kemen E, et al. (2009). A new species of Albugo parasitic to Arabidopsis thaliana reveals new evolutionary patterns in white blister rusts (Albuginaceae). Persoonia; 22:123.

15. Cooper AJ, Latunde-Dada AO, Woods-Tör A, et al. (2008). Basic compatibility of Albugo candida in Arabidopsis thaliana and Brassica juncea causes broadspectrum suppression of innate immunity. Mol Plant-Microbe Interact; 21:745756. 
16. Prince DC, Rallapalli G, Xu D, et al. (2017). Albugo-imposed changes to tryptophan-derived antimicrobial metabolite biosynthesis may contribute to suppression of non-host resistance to Phytophthora infestans in Arabidopsis thaliana. BMC Biology; 15:20.

17. Thines M, Choi YJ. (2016). Evolution, diversity, and taxonomy of the Peronosporaceae, with focus on the genus Peronospora. Phytopathology; 106:6-18.

18. Levesque CA, Brouwer H, Cano L, et al. (2010). Genome sequence of the necrotrophic plant pathogen Pythium ultimum reveals original pathogenicity mechanisms and effector repertoire. Genome Biol; 11:R73.

19. McCarthy CGP, Fitzpatrick DA. (2017). Phylogenomic reconstruction of the oomycete phylogeny derived from 37 genomes. mSphere; 2.

20. Ascunce MS, Huguet-Tapia JC, Ortiz-Urquiza A, et al. (2017). Phylogenomic analysis supports multiple instances of polyphyly in the oomycete peronosporalean lineage. Molecular Phylogenetics and Evolution; 114:199-211.

21. Turner RS. (2005). After the famine: Plant pathology, Phytophthora infestans, and the late blight of potatoes, 1845-1960. Historical Studies in the Physical and Biological Sciences; 35:341-370.

22. Dussert Y, Mazet ID, Couture C, et al. (2019). A high-quality grapevine downy mildew genome assembly reveals rapidly evolving and lineage-specific putative host adaptation genes. Genome Biol Evol; 11:954-969.

23. Correll JC, Bluhm BH, Feng C, et al. (2011). Spinach: better management of downy mildew and white rust through genomics. European Journal of Plant Pathology; 129:193-205.

24. Beakes GW, Glockling SL, Sekimoto S. (2012). The evolutionary phylogeny of the oomycete "fungi". Protoplasma; 249:3-19.

25. Thines M. (2009). Bridging the gulf: Phytophthora and downy mildews are connected by rare grass parasites. PLOS ONE; 4:e4790-e4790.

26. Ye W, Wang Y, Shen D, et al. (2016). Sequencing of the Litchi Downy Blight Pathogen Reveals It Is a Phytophthora Species With Downy Mildew-Like Characteristics. Molecular Plant-Microbe Interactions; 29:573-583.

27. Bourret TB, Choudhury RA, Mehl HK, et al. (2018). Multiple origins of downy mildews and mito-nuclear discordance within the paraphyletic genus Phytophthora. PLOS ONE; 13:e0192502.

28. Stassen JHM, Seidl MF, Vergeer PWJ, et al. (2012). Effector identification in the lettuce downy mildew Bremia lactucae by massively parallel transcriptome sequencing. Molecular Plant Pathology; 13:719-731.

29. Sharma R, Xia X, Cano LM, et al. (2015). Genome analyses of the sunflower pathogen Plasmopara halstedii provide insights into effector evolution in downy mildews and Phytophthora. BMC Genomics; 16:741. 
30. Kandel SL, Mou B, Shishkoff N, et al. (2019). Spinach downy mildew: advances in our understanding of the disease cycle and prospects for disease management. Plant Dis; 103:791-803.

31. Greville RK. Flora Edinensis. 1824.

32. Ribera A, Bai Y, Wolters A-MA, et al. (2020). A review on the genetic resources, domestication and breeding history of spinach (Spinacia oleracea L.). Euphytica; 216:48.

33. Inaba T, Morinaka T. (1984). Heterothallism in Peronospora effusa. Phytopathology; 74:214-216.

34. Van Asch M, Frinking H. (1988). Heterothallism in Peronospora farinosa f. sp. spinaciae. Transactions of the british mycological society; 91:692-693.

35. Kunjeti SG, Anchieta A, Subbarao KV, et al. (2016). Plasmolysis and vital staining reveal viable oospores of Peronospora effusa in spinach seed lots. Plant Dis; 100:59-65.

36. Manners J, Gay J. (1983). The host-parasite interface and nutrient transfer in biotrophic parasitism. Biochemical plant pathology:163-195.

37. Hardham AR. (2007). Cell biology of plant-oomycete interactions. Cellular Microbiology; 9:31-39.

38. Caillaud M-C, Wirthmueller L, Sklenar J, et al. (2014). The plasmodesmal protein PDLP1 localises to haustoria-associated membranes during downy mildew infection and regulates callose deposition. PLOS pathogens; 10:e1004496.

39. Raaymakers TM, Van den Ackerveken G. (2016). Extracellular recognition of oomycetes during biotrophic infection of plants. Front Plant Sci; 7:906 - 917.

40. Kamoun S. (2006). A catalogue of the effector secretome of plant pathogenic oomycetes. Annu Rev Phytopathol; 44:41-60.

41. Schwessinger B, Zipfel C. (2008). News from the frontline: recent insights into PAMP-triggered immunity in plants. Current Opinion in Plant Biology; 11:389395.

42. Altenbach D, Robatzek S. (2007). Pattern Recognition Receptors: From the Cell Surface to Intracellular Dynamics. Mol Plant Microbe Interact; 20:10311039.

43. Tsuda K, Katagiri F. (2010). Comparing signaling mechanisms engaged in pattern-triggered and effector-triggered immunity. Current Opinion in Plant Biology; 13:459-465.

44. Rocafort M, Fudal I, Mesarich CH. (2020). Apoplastic effector proteins of plantassociated fungi and oomycetes. Current Opinion in Plant Biology; 56:9-19.

45. Rovenich H, Zuccaro A, Thomma BPHJ. (2016). Convergent evolution of filamentous microbes towards evasion of glycan-triggered immunity. New Phytologist; 212:896-901.

46. Ruiz-Herrera J, Ortiz-Castellanos L. (2019). Cell wall glucans of fungi. A review. The Cell Surface; 5:100022. 
47. Fesel PH, Zuccaro A. (2016). $\beta$-glucan: Crucial component of the fungal cell wall and elusive MAMP in plants. Fungal Genetics and Biology; 90:53-60.

48. Tian M, Win J, Song J, et al. (2007). A Phytophthora infestans cystatin-like protein targets a novel tomato papain-like apoplastic protease. Plant Physiol; 143:364-77.

49. Mikes V, Milat M-L, Ponchet M, et al. (1998). Elicitins, proteinaceous elicitors of plant defense, are a new class of sterol carrier proteins. Biochemical and biophysical research communications; 245:133-139.

50. Nine things to know about elicitins, (2016).

51. Seidl MF, Van den Ackerveken G. (2019). Activity and Phylogenetics of the Broadly Occurring Family of Microbial Nep1-Like Proteins. Annual Review of Phytopathology; 57:367-386.

52. Cabral A, Oome S, Sander N, et al. (2012). Nontoxic Nep1-like proteins of the downy mildew pathogen Hyaloperonospora arabidopsidis: repression of necrosis-inducing activity by a surface-exposed region. Mol Plant-Microbe Interact; 25:697-708.

53. Seidl MF, Van Den Ackerveken G, Govers F, Snel B. (2011). A Domain-Centric Analysis of Oomycete Plant Pathogen Genomes Reveals Unique Protein Organization. Plant Physiol; 155:628-44.

54. Kanneganti T-D, Huitema E, Cakir C, Kamoun S. (2006). Synergistic interactions of the plant cell death pathways induced by Phytophthora infestans Nep1-like protein PiNPP1. 1 and INF1 elicitin. Molecular plant-microbe interactions; 19:854-863.

55. Rehmany AP, Gordon A, Rose LE, et al. (2005). Differential recognition of highly divergent downy mildew avirulence gene alleles by RPP1 resistance genes from two Arabidopsis lines. The Plant Cell; 17:1839-1850.

56. Schornack S, van Damme M, Bozkurt TO, et al. (2010). Ancient class of translocated oomycete effectors targets the host nucleus. Proc Natl Acad Sci U $S A ; 107: 17421-6$.

57. Schornack S, Huitema E, Cano LM, et al. (2009). Ten things to know about oomycete effectors. Mol Plant Pathol; 10:795-803.

58. Haas BJ, Kamoun S, Zody MC, et al. (2009). Genome sequence and analysis of the Irish potato famine pathogen Phytophthora infestans. Nature; 461:393-8.

59. Baxter L, Tripathy S, Ishaque N, et al. (2010). Signatures of adaptation to obligate biotrophy in the Hyaloperonospora arabidopsidis genome. Science; 330:1549-1551.

60. Derevnina L, Chin-Wo-Reyes S, Martin F, et al. (2015). Genome sequence and architecture of the tobacco downy mildew pathogen Peronospora tabacina. Mol Plant-Microbe Interact; 28:1198-215.

61. Fletcher K, Gil J, Bertier LD, et al. (2019). Genomic signatures of somatic hybrid vigor due to heterokaryosis in the oomycete pathogen, Bremia lactucae. bioRxiv:516526. 
62. Thines M, Sharma R, Rodenburg SYA, et al. (2020). The Genome of Peronospora belbahrii reveals high heterozygosity, a low number of canonical effectors, and TC-rich promoters. Molecular Plant-Microbe Interactions; 33:742753.

63. Kemen E, Gardiner A, Schultz-Larsen T, et al. (2011). Gene gain and loss during evolution of obligate parasitism in the white rust pathogen of Arabidopsis thaliana. PLOS Biol; 9:e1001094.

64. Ai G, Yang K, Ye W, et al. (2020). Prediction and Characterization of RXLR Effectors in Pythium Species. Molecular Plant-Microbe Interactions.

65. Whisson SC, Boevink PC, Moleleki L, et al. (2007). A translocation signal for delivery of oomycete effector proteins into host plant cells. Nature; 450:115-118.

66. Anderson RG, Deb D, Fedkenheuer K, McDowell JM. (2015). Recent Progress in RXLR Effector Research. Mol Plant-Microbe Interact; 28:1063-72.

67. Dou D, Kale SD, Wang X, et al. (2008). RXLR-mediated entry of Phytophthora sojae effector Avr1b into soybean cells does not require pathogen-encoded machinery. The Plant Cell; 20:1930-1947.

68. Driouich A, Jauneau A, Staehelin LA. (1997). 7-Dehydrobrefeldin A, a naturally occurring brefeldin A derivative, inhibits secretion and causes a cis-totrans breakdown of Golgi stacks in plant cells. Plant physiology; 113:487-492.

69. Wang S, Boevink PC, Welsh L, et al. (2017). Delivery of cytoplasmic and apoplastic effectors from Phytophthora infestans haustoria by distinct secretion pathways. New Phytol; 216:205-215.

70. Giraldo MC, Dagdas YF, Gupta YK, et al. (2013). Two distinct secretion systems facilitate tissue invasion by the rice blast fungus Magnaporthe oryzae. Nature communications; 4:1-12.

71. Van den Ackerveken G. (2017). Seeing is believing: imaging the delivery of pathogen effectors during plant infection. New Phytologist; 216:8-10.

72. Micali CO, Neumann U, Grunewald D, et al. (2011). Biogenesis of a specialized plant-fungal interface during host cell internalization of Golovinomyces orontii haustoria. Cellular Microbiology; 13:210-226.

73. Koh S, André A, Edwards H, et al. (2005). Arabidopsis thaliana subcellular responses to compatible Erysiphe cichoracearum infections. The Plant Journal; 44:516-529.

74. Lo Presti L, Kahmann R. (2017). How filamentous plant pathogen effectors are translocated to host cells. Curr Opin Plant Biol; 38:19-24.

75. Win J, Morgan W, Bos J, et al. (2007). Adaptive evolution has targeted the C-terminal domain of the RXLR effectors of plant pathogenic oomycetes. Plant Cell; 19:2349-69.

76. Mukhi N, Gorenkin D, Banfield MJ. (2020). Exploring folds, evolution and host interactions: understanding effector structure/function in disease and immunity. New Phytol. 
77. Boutemy LS, King SR, Win J, et al. (2011). Structures of Phytophthora RXLR effector proteins: a conserved but adaptable fold underpins functional diversity. J Biol Chem; 286:35834-42.

78. Maqbool A, Hughes RK, Dagdas YF, et al. (2016). Structural basis of host autophagy-related protein 8 (ATG8) binding by the Irish potato famine pathogen effector protein PexRD54. Journal of Biological Chemistry; 291:20270-20282.

79. He J, Ye W, Choi DS, et al. (2019). Structural analysis of Phytophthora suppressor of RNA silencing 2 (PSR2) reveals a conserved modular fold contributing to virulence. Proc Natl Acad Sci; 116:8054-8059.

80. Dodds PN, Rathjen JP. (2010). Plant immunity: towards an integrated view of plant-pathogen interactions. Nature Reviews Genetics; 11:539-548.

81. Rajput NA, Zhang M, Ru Y, et al. (2014). Phytophthora sojae effector PsCRN70 suppresses plant defenses in Nicotiana benthamiana. PLOS ONE; 9:e98114-e98114.

82. King SR, McLellan H, Boevink PC, et al. (2014). Phytophthora infestans RXLR effector PexRD2 interacts with host MAPKKK epsilon to suppress plant immune signaling. Plant Cell; 26:1345-59.

83. He Q, McLellan H, Hughes RK, et al. (2019). Phytophthora infestans effector SFI3 targets potato UBK to suppress early immune transcriptional responses. New Phytologist; 222:438-454.

84. He Q, Naqvi S, McLellan H, et al. (2018). Plant pathogen effector utilizes host susceptibility factor NRL1 to degrade the immune regulator SWAP70. Proceedings of the National Academy of Sciences; 115.

85. Fan G, Yang Y, Li T, et al. (2018). A Phytophthora capsici RXLR effector targets and inhibits a plant PPlase to suppress endoplasmic reticulum-mediated immunity. Molecular plant; 11:1067-1083.

86. McLellan H, Boevink PC, Armstrong MR, et al. (2013). An RxLR effector from Phytophthora infestans prevents re-localisation of two plant NAC transcription factors from the endoplasmic reticulum to the nucleus. PLOS Pathog; 9:e1003670.

87. Jing M, Guo B, Li H, et al. (2016). A Phytophthora sojae effector suppresses endoplasmic reticulum stress-mediated immunity by stabilizing plant Binding immunoglobulin Proteins. Nature Communications; 7:11685.

88. Bouwmeester K, de Sain M, Weide R, et al. (2011). The lectin receptor kinase LecRK-I.9 is a novel Phytophthora resistance component and a potential host target for a RXLR effector. PLOS pathogens; 7:e1001327-e1001327.

89. Dong S, Yin W, Kong G, et al. (2011). Phytophthora sojae Avirulence Effector Avr3b is a Secreted NADH and ADP-ribose Pyrophosphorylase that Modulates Plant Immunity. PLOS Pathogens; 7:e1002353.

90. Boevink PC, Wang X, McLellan H, et al. (2016). A Phytophthora infestans $\mathrm{RXLR}$ effector targets plant PP1c isoforms that promote late blight disease. Nature Communications; 7:10311. 
91. Caillaud MC, Asai S, Rallapalli G, et al. (2013). A downy mildew effector attenuates salicylic acid-triggered immunity in Arabidopsis by interacting with the host mediator complex. PLoS Biol; 11:e1001732.

92. Turnbull D, Yang L, Naqvi S, et al. (2017). RXLR Effector AVR2 Up-Regulates a Brassinosteroid-Responsive bHLH Transcription Factor to Suppress Immunity. Plant Physiology; 174:356.

93. Anderson R, Deb D, Withers J, et al. (2019). An oomycete RXLR effector triggers antagonistic plant hormone crosstalk to suppress host immunity. bioRxiv.

94. Yang B, Wang Y, Guo B, et al. (2019). The Phytophthora sojae RXLR effector Avh238 destabilizes soybean Type2 Gm ACS s to suppress ethylene biosynthesis and promote infection. New Phytologist; 222:425-437.

95. Zhang P, Jia Y, Shi J, et al. (2019). The WY domain in the Phytophthora effector PSR1 is required for infection and RNA silencing suppression activity. New Phytol; 223:839-852.

96. Harvey S, Kumari P, Lapin D, et al. (2020). Downy Mildew effector HaRxL21 interacts with the transcriptional repressor TOPLESS to promote pathogen susceptibility. bioRxiv.

97. Song T, Ma Z, Shen D, et al. (2015). An Oomycete CRN Effector Reprograms Expression of Plant HSP Genes by Targeting their Promoters. PLOS pathogens; 11:e1005348-e1005348.

98. Kong L, Qiu X, Kang J, et al. (2017). A Phytophthora effector manipulates host histone acetylation and reprograms defense gene expression to promote infection. Current Biology; 27:981-991.

99. Wang X, Boevink P, McLellan H, et al. (2015). A Host KH RNA-Binding Protein Is a Susceptibility Factor Targeted by an RXLR Effector to Promote Late Blight Disease. Molecular Plant; 8:1385-1395.

100. Huang J, Gu L, Zhang Y, et al. (2017). An oomycete plant pathogen reprograms host pre-mRNA splicing to subvert immunity. Nature communications; 8:1-15.

101. Liu T, Ye W, Ru Y, et al. (2011). Two host cytoplasmic effectors are required for pathogenesis of Phytophthora sojae by suppression of host defenses. Plant physiology; 155:490-501.

102. Li Q, Zhang M, Shen D, et al. (2016). A Phytophthora sojae effector PsCRN63 forms homo-/hetero-dimers to suppress plant immunity via an inverted association manner. Scientific reports; 6 .

103. Zhang M, Li Q, Liu T, et al. (2015). Two Cytoplasmic Effectors of Phytophthora sojae Regulate Plant Cell Death via Interactions with Plant Catalases. Plant Physiology; 167:164-175.

104. Bozkurt TO, Schornack S, Win J, et al. (2011). Phytophthora infestans effector AVRblb2 prevents secretion of a plant immune protease at the haustorial interface. Proc Natl Acad Sci U S A; 108:20832-7. 
105. Chaparro-Garcia A, Schwizer S, Sklenar J, et al. (2015). Phytophthora infestans RXLR-WY Effector AVR3a Associates with Dynamin-Related Protein 2 Required for Endocytosis of the Plant Pattern Recognition Receptor FLS2. PLOS ONE; 10:e0137071-e0137071.

106. Dagdas YF, Belhaj K, Maqbool A, et al. (2016). An effector of the Irish potato famine pathogen antagonizes a host autophagy cargo receptor. Elife; 5:e10856.

107. Du Y, Mpina MH, Birch PR, et al. (2015). Phytophthora infestans RXLR Effector AVR1 Interacts with Exocyst Component Sec5 to Manipulate Plant Immunity. Plant Physiol; 169:1975-90.

108. Yamaguchi K, Kawasaki T. (2012). Function of Arabidopsis SWAP70 GEF in immune response. Plant Signaling \& Behavior; 7:465-468.

109. Yang L, McLellan H, Naqvi S, et al. (2016). Potato NPH3/RPT2-Like Protein StNRL1, Targeted by a Phytophthora infestans RXLR Effector, Is a Susceptibility Factor. Plant Physiol; 171:645-57.

110. Xiong Q, Ye W, Choi D, et al. (2014). Phytophthora suppressor of RNA silencing 2 is a conserved RxLR effector that promotes infection in soybean and Arabidopsis thaliana. Mol Plant Microbe Interact; 27:1379-89.

111. Lusser A, Kölle D, Loidl P. (2001). Histone acetylation: lessons from the plant kingdom. Trends in plant science; 6:59-65.

112. Mhamdi A, Queval G, Chaouch S, et al. (2010). Catalase function in plants: a focus on Arabidopsis mutants as stress-mimic models. Journal of Experimental Botany; 61:4197-4220.

113. Robatzek S. (2007). Vesicle trafficking in plant immune responses. Cellular Microbiology; 9:1-8.

114. Felix G, Duran JD, Volko S, Boller T. (1999). Plants have a sensitive perception system for the most conserved domain of bacterial flagellin. The Plant Journal; 18:265-276.

115. Robatzek S, Chinchilla D, Boller T. (2006). Ligand-induced endocytosis of the pattern recognition receptor FLS2 in Arabidopsis. Genes \& development; 20:537-542.

116. Koike S, Smith R, Schulbach K. (1992). Resistant cultivars, fungicides combat downy mildew of spinach. California Agriculture; 46:29-30.

117. Wang S, Welsh L, Thorpe P, et al. (2018). The Phytophthora infestans haustorium is a site for secretion of diverse classes of infection-associated proteins. MBio; 9:1216-18.

118. Ellis JG, Rafiqi M, Gan P, et al. (2009). Recent progress in discovery and functional analysis of effector proteins of fungal and oomycete plant pathogens. Curr Opin Plant Biol; 12:399-405.

119. Deb D, Anderson RG, How-Yew-Kin T, et al. (2018). Conserved RxLR effectors from oomycetes Hyaloperonospora arabidopsidis and Phytophthora sojae suppress PAMP- and Effector-Triggered Immunity in diverse plants. Mol Plant-Microbe Interact; 31:374-385. 
120. Dou D, Kale SD, Wang X, et al. (2008). Conserved C-terminal motifs required for avirulence and suppression of cell death by Phytophthora sojae effector Avr1b. The Plant Cell; 20:1118-1133.

121. Rehmany AP, Gordon A, Rose LE, et al. (2005). Differential recognition of highly divergent downy mildew avirulence gene alleles by RPP1 resistance genes from two Arabidopsis lines. Plant Cell; 17:1839-50.

122. von Meijenfeldt FAB, Arkhipova K, Cambuy DD, et al. (2019). Robust taxonomic classification of uncharted microbial sequences and bins with CAT and BAT. Genome Biol; 20:217.

123. Schmieder R, Edwards R. (2011). Quality control and preprocessing of metagenomic datasets. Bioinformatics; 27:863-864.

124. Chin CS, Peluso P, Sedlazeck FJ, et al. (2016). Phased diploid genome assembly with single-molecule real-time sequencing. Nat Methods; 13:10501054.

125. (2019). SMRT Analysis Software. PacBio.

126. Hyatt D, Chen GL, Locascio PF, et al. (2010). Prodigal: prokaryotic gene recognition and translation initiation site identification. Bmc Bioinformatics; 11:119.

127. Buchfink B, Xie C, Huson DH. (2014). Fast and sensitive protein alignment using DIAMOND. Nature Methods; 12:59.

128. Bankevich A, Nurk S, Antipov D, et al. (2012). SPAdes: a new genome assembly algorithm and its applications to single-cell sequencing. $J$ Comput Biol; 19:455-77.

129. Langmead B, Trapnell C, Pop M, Salzberg SL. (2009). Ultrafast and memoryefficient alignment of short DNA sequences to the human genome. Genome Biol; 10:R25.

130. Smit A, Hubley R, Green P. (2015). RepeatMasker Open-4.0. 2013-2015. http://www.repeatmasker.org.

131. Marcais G, Kingsford C. (2011). A fast, lock-free approach for efficient parallel counting of occurrences of k-mers. Bioinformatics; 27:764-70.

132. Vurture GW, Sedlazeck FJ, Nattestad M, et al. (2017). GenomeScope: fast reference-free genome profiling from short reads. Bioinformatics; 33:2202-2204.

133. Li H. (2013). Aligning sequence reads, clone sequences and assembly contigs with BWA-MEM. arXiv preprint arXiv:13033997.

134. Bushnell B. (2014). BBMap: a fast, accurate, splice-aware aligner. United States: Lawrence Berkeley National Lab. (LBNL).

135. Meneghin J. (2009). Get GC Content (Perl script). https://github.com/CostaLab/ practical_SS2015/.

136. Wickham H. ggplot2: elegant graphics for data analysis. Springer; 2016. p. VIII 213. 
137. Thines M, Sharma R, Rodenburg SYA, et al. (2019). The genome of Peronospora belbahrii reveals high heterozygosity, a low number of canonical effectors and CT-rich promoters. bioRxiv:721027.

138. Tyler BM, Tripathy S, Zhang X, et al. (2006). Phytophthora genome sequences uncover evolutionary origins and mechanisms of pathogenesis. Science; 313:1261-6.

139. Menzel P, Ng KL, Krogh A. (2016). Fast and sensitive taxonomic classification for metagenomics with Kaiju. Nat Commun; 7:11257.

140. Huang W, Li L, Myers JR, Marth GT. (2012). ART: a next-generation sequencing read simulator. Bioinformatics; 28:593-594.

141. Simao FA, Waterhouse RM, loannidis P, et al. (2015). BUSCO: assessing genome assembly and annotation completeness with single-copy orthologs. Bioinformatics; 31:3210-2.

142. Trapnell C, Pachter L, Salzberg SL. (2009). TopHat: discovering splice junctions with RNA-Seq. Bioinformatics; 25:1105-1111.

143. Hoff KJ, Lange S, Lomsadze A, et al. (2016). BRAKER1: Unsupervised RNA-Seq-Based Genome Annotation with GeneMark-ET and AUGUSTUS. Bioinformatics; 32:767-9.

144. Lee E, Helt GA, Reese JT, et al. (2013). Web Apollo: a web-based genomic annotation editing platform. Genome Biol; 14.

145. Ooi HS, Kwo CY, Wildpaner M, et al. (2009). ANNIE: integrated de novo protein sequence annotation. Nucleic Acids Res; 37:W435-40.

146. Finn RD, Coggill P, Eberhardt RY, et al. (2016). The Pfam protein families database: towards a more sustainable future. Nucleic Acids Res; 44:D279-85.

147. Petersen TN, Brunak S, von Heijne G, Nielsen H. (2011). SignalP 4.0: discriminating signal peptides from transmembrane regions. Nat Methods; 8:785-6.

148. Sperschneider J, Williams AH, Hane JK, et al. (2015). Evaluation of secretion prediction highlights differing approaches needed for oomycete and fungal effectors [Original Research]. Front Plant Sci; 6:1168.

149. Krogh A, Larsson B, von Heijne G, Sonnhammer ELL. (2001). Predicting transmembrane protein topology with a hidden markov model: application to complete genomes. Journal of Molecular Biology; 305:567-580.

150. Win J, Krasileva KV, Kamoun S, et al. (2012). Sequence divergent RXLR effectors share a structural fold conserved across plant pathogenic oomycete species. PLOS Pathog; 8:e1002400.

151. Eddy SR. (1998). Profile hidden Markov models. Bioinformatics (Oxford, England); 14:755-763.

152. Klein J. (2018). GitHub repository, https://github.com/kleinjoel/bioscripts/.

153. Stajich JE, Block D, Boulez K, et al. (2002). The Bioperl toolkit: Perl modules for the life sciences. Genome research; 12:1611-1618. 
154. Johnson LS, Eddy SR, Portugaly E. (2010). Hidden Markov model speed heuristic and iterative HMM search procedure. Bmc Bioinformatics; 11:431.

155. Armitage AD, Lysøe E, Nellist CF, et al. (2018). Bioinformatic characterisation of the effector repertoire of the strawberry pathogen Phytophthora cactorum. PLOS ONE; 13:e0202305.

156. Wilcoxon F, Katti S, Wilcox RA. Critical values and probability levels for the Wilcoxon rank sum test and the Wilcoxon signed rank test. Selected tables in mathematical statistics. Vol. 11970. p. 171-259.

157. Jones P, Binns D, Chang H-Y, et al. (2014). InterProScan 5: genome-scale protein function classification. Bioinformatics; 30:1236-1240.

158. Cantarel BL, Coutinho PM, Rancurel C, et al. (2009). The CarbohydrateActive EnZymes database (CAZy): an expert resource for Glycogenomics. Nucleic Acids Research; 37:D233-D238.

159. Zhang $\mathbf{H}$, Yohe $\mathbf{T}$, Huang $\mathbf{L}$, et al. (2018). dbCAN2: a meta server for automated carbohydrate-active enzyme annotation. Nucleic Acids Research; 46:W95-W101.

160. Emms DM, Kelly S. (2015). OrthoFinder: solving fundamental biases in whole genome comparisons dramatically improves orthogroup inference accuracy. Genome Biology; 16:157.

161. Katoh K, Standley DM. (2013). MAFFT Multiple Sequence Alignment Software Version 7: Improvements in performance and usability. Molecular Biology and Evolution; 30:772-780.

162. Lefort V, Desper R, Gascuel O. (2015). FastME 2.0: A comprehensive, accurate, and fast distance-based phylogeny inference program. Molecular Biology and Evolution; 32:2798-2800.

163. He Z, Zhang H, Gao S, et al. (2016). Evolview v2: an online visualization and management tool for customized and annotated phylogenetic trees. Nucleic acids research; 44:W236-W241.

164. McMurdie PJ, Holmes S. (2013). phyloseq: An R package for reproducible interactive analysis and graphics of microbiome census data. PLOS ONE; 8:e61217.

165. Team R. (2013). R: A language and environment for statistical computing.

166. Team R. (2015). RStudio: integrated development for R. RStudio, Inc., Boston, MA http://www.rstudio.com.

167. Dixon P. (2003). VEGAN, a package of R functions for community ecology. Journal of Vegetation Science; 14:927-930.

168. Lyon R, Correll J, Feng C, et al. (2016). Population structure of peronospora effusa in the southwestern United States. PLOS ONE; 11:e0148385.

169. Meisrimler CN, Pelgrom AJE, Oud B, et al. (2019). Multiple downy mildew effectors target the stress-related NAC transcription factor LsNAC069 in lettuce. Plant J; 0:1098-1115. 
170. Boutemy LS, King SRF, Win J, et al. (2011). Structures of Phytophthora RXLR Effector Proteins: A CONSERVED BUT ADAPTABLE FOLD UNDERPINS FUNCTIONAL DIVERSITY. The Journal of Biological Chemistry; 286:3583435842.

171. Jiang RHY, Tripathy S, Govers F, Tyler BM. (2008). RXLR effector reservoir in two Phytophthora species is dominated by a single rapidly evolving superfamily with more than 700 members. Proceedings of the National Academy of Sciences of the United States of America; 105:4874-4879.

172. Dong S, Raffaele S, Kamoun S. (2015). The two-speed genomes of filamentous pathogens: waltz with plants. Curr Opin Genet Dev; 35:57-65.

173. McGowan J, Fitzpatrick DA. (2017). Genomic, network, and phylogenetic analysis of the oomycete effector arsenal. $m$ Sphere; 2:408-17.

174. Blackman LM, Cullerne DP, Hardham AR. (2014). Bioinformatic characterisation of genes encoding cell wall degrading enzymes in the Phytophthora parasitica genome. BMC Genomics; 15:785.

175. Fellbrich G, Romanski A, Varet A, et al. (2002). NPP1, a Phytophthoraassociated trigger of plant defense in parsley and Arabidopsis. Plant Journal; 32:375-390.

176. Seidl MF, Van den Ackerveken G. (2019). Activity and phylogenetics of the broadly occurring family of microbial Nep1-Like proteins. Annual review of phytopathology; 57.

177. Ottmann C, Luberacki B, Küfner I, et al. (2009). A common toxin fold mediates microbial attack and plant defense. Proceedings of the National Academy of Sciences of the United States of America; 106:10359-10364.

178. Mittl PR, Schneider-Brachert W. (2007). Sel1-like repeat proteins in signal transduction. Cellular signalling; 19:20-31.

179. Larroque $\mathbf{M}$, Barriot R, Bottin A, et al. (2012). The unique architecture and function of cellulose-interacting proteins in oomycetes revealed by genomic and structural analyses. BMC Genomics; 13:605.

180. Hu G, Leger RJS. (2004). A phylogenomic approach to reconstructing the diversification of serine proteases in fungi. Journal of Evolutionary Biology; 17:1204-1214.

181. Jashni MK, Dols IHM, lida Y, et al. (2015). Synergistic action of a metalloprotease and a serine protease from Fusarium oxysporum f. sp. Lycopersici cleaves chitin-binding tomato chitinases, reduces their antifungal activity, and enhances fungal virulence. Mol Plant-Microbe Interact; 28:996-1008.

182. Adhikari BN, Hamilton JP, Zerillo MM, et al. (2013). Comparative genomics reveals insight into virulence strategies of plant pathogenic oomycetes. PLoS One; 8:e75072.

183. McGowan J, Byrne KP, Fitzpatrick DA. (2018). Comparative analysis of oomycete genome evolution using the oomycete gene order browser (OGOB). Genome biology and evolution; 11:189-206. 
184. Bohlin J, Eldholm V, Pettersson JH, et al. (2017). The nucleotide composition of microbial genomes indicates differential patterns of selection on core and accessory genomes. BMC Genomics; 18:151.

185. Lamour K, Kamoun S. Oomycete genetics and genomics: diversity, interactions and research tools. John Wiley \& Sons; 2009. p. 592.

186. De Bustos A, Cuadrado A, Jouve N. (2016). Sequencing of long stretches of repetitive DNA. Scientific reports; 6:36665.

187. Fletcher K, Klosterman SJ, Derevnina L, et al. (2018). Comparative genomics of downy mildews reveals potential adaptations to biotrophy. BMC Genomics; 19:851-884.

188. Feng C, Lamour KH, Bluhm BH, et al. (2018). Genome sequences of three races of Peronospora effusa: a resource for studying the evolution of the spinach downy mildew pathogen. Mol Plant-Microbe Interact; 31:1230-1231.

189. Soanes D, Richards TA. (2014). Horizontal gene transfer in eukaryotic plant pathogens. Annual Review of Phytopathology; 52:583-614.

190. Raffaele S, Kamoun S. (2012). Genome evolution in filamentous plant pathogens: why bigger can be better [Review Article]. Nat Rev Microbiol; 10:41730.

191. Seidl MF, van den Ackerveken G, Govers F, Snel B. (2012). Reconstruction of oomycete genome evolution identifies differences in evolutionary trajectories leading to present-day large gene families. Genome Biology and Evolution; 4:199-211.

192. Cui C, Herlihy JH, Bombarely A, et al. (2019). Draft Assembly of Phytophthora capsici from Long-Read Sequencing Uncovers Complexity. Mol Plant Microbe Interact; 32:1559-1563.

193. Sharma R, Xia X, Cano LM, et al. (2015). Genome analyses of the sunflower pathogen Plasmopara halstedii provide insights into effector evolution in downy mildews and Phytophthora. BMC Genomics; 16:741.

194. Latijnhouwers M, de Wit PJ, Govers F. (2003). Oomycetes and fungi: Similar weaponry to attack plants. Trends Microbiol; 11:462-9.

195. Richards TA, Dacks JB, Jenkinson JM, et al. (2006). Evolution of filamentous plant pathogens: gene exchange across eukaryotic kingdoms. Curr Biol; 16:1857-64.

196. Richards TA, Soanes DM, Jones MDM, et al. (2011). Horizontal gene transfer facilitated the evolution of plant parasitic mechanisms in the oomycetes. Proceedings of the National Academy of Sciences of the United States of America; 108:15258-15263.

197. Kemen E, Jones JDG. (2012). Obligate biotroph parasitism: Can we link genomes to lifestyles? Trends in Plant Science; 17:448-457.

198. Boller T, He SY. (2009). Innate immunity in plants: an arms race between pattern recognition receptors in plants and effectors in microbial pathogens. Science; 324:742-744. 
199. Sánchez-Vallet A, Fouché S, Fudal I, et al. (2018). The Genome Biology of Effector Gene Evolution in Filamentous Plant Pathogens. Annual Review of Phytopathology; 56:21-40.

200. Birch PR, Boevink PC, Gilroy EM, et al. (2008). Oomycete RXLR effectors: delivery, functional redundancy and durable disease resistance. Curr Opin Plant Biol; 11:373-9.

201. Qiao Y, Shi J, Zhai Y, et al. (2015). Phytophthora effector targets a novel component of small RNA pathway in plants to promote infection. Proceedings of the National Academy of Sciences; 112:5850-5855.

202. Varden FA, De La Concepcion JC, Maidment JH, Banfield MJ. (2017). Taking the stage: effectors in the spotlight. Current Opinion in Plant Biology; 38:25-33.

203. Yin L, An Y, Qu J, et al. (2017). Genome sequence of Plasmopara viticola and insight into the pathogenic mechanism. Scientific Reports; 7:46553.

204. Anderson RG, Casady MS, Fee RA, et al. (2012). Homologous RXLR effectors from Hyaloperonospora arabidopsidis and Phytophthora sojae suppress immunity in distantly related plants. Plant J; 72:882-93.

205. Weßling R, Epple P, Altmann S, et al. (2014). Convergent Targeting of a Common Host Protein-Network by Pathogen Effectors from Three Kingdoms of Life. Cell Host \& Microbe; 16:364-375.

206. Mukhtar MS, Carvunis AR, Dreze M, et al. (2011). Independently evolved virulence effectors converge onto hubs in a plant immune system network. Science; 333:596-601.

207. Klein J. (2019). Genome analyses of resistance-breaking isolates of the spinach downy mildew Peronospora effusa. Thesis. Universiteit Utrecht.

208. Nguyen Ba AN, Pogoutse A, Provart N, Moses AM. (2009). NLStradamus: a simple Hidden Markov Model for nuclear localization signal prediction. Bmc Bioinformatics; 10:202.

209. Caillaud M-C, Piquerez SJM, Fabro G, et al. (2012). Subcellular localization of the Hpa RxLR effector repertoire identifies a tonoplast-associated protein HaRxL17 that confers enhanced plant susceptibility. Plant J; 69:252-265.

210. Caillaud M-C, Wirthmueller L, Fabro G, et al. (2012). Mechanisms of nuclear suppression of host immunity by effectors from the Arabidopsis downy mildew pathogen Hyaloperonospora arabidopsidis (Hpa). Cold Spring Harbor Symposia on Quantitative Biology; 77:285-293.

211. Kelly S, Maini PK. (2013). DendroBLAST: approximate phylogenetic trees in the absence of multiple sequence alignments. PLOS ONE; 8.

212. Letunic I, Bork P. (2019). Interactive Tree Of Life (iTOL) v4: recent updates and new developments. Nucleic Acids Research; 47:W256-W259.

213. Von Mering C, Krause R, Snel B, et al. (2002). Comparative assessment of large-scale data sets of protein-protein interactions. Nature; 417:399-403. 
214. González-Fuente M, Carrère S, Monachello D, et al. (2019). EffectorK, a comprehensive resource to mine for pathogen effector targets in the Arabidopsis proteome. Cold Spring Harbor Laboratory.

215. Stark C. (2006). BioGRID: a general repository for interaction datasets. Nucleic Acids Research; 34:D535-D539.

216. Orchard S, Ammari M, Aranda B, et al. (2014). The MlntAct project-IntAct as a common curation platform for 11 molecular interaction databases. Nucleic Acids Research; 42:D358-D363.

217. Dreze M, Carvunis AR, Charloteaux B, et al. (2011). Evidence for Network Evolution in an Arabidopsis Interactome Map. Science; 333:601-607.

218. Smakowska-Luzan E, Mott GA, Parys K, et al. (2018). An extracellular network of Arabidopsis leucine-rich repeat receptor kinases. Nature; 553:342-346.

219. Wessling R, Epple P, Altmann S, et al. (2014). Convergent targeting of a common host protein-network by pathogen effectors from three kingdoms of life. Cell Host Microbe; 16:364-75.

220. Singh AK, Chamovitz DA. (2019). Role of Cop9 Signalosome Subunits in the Environmental and Hormonal Balance of Plant [Review]. Biomolecules; 9:224.

221. Hind SR, Pulliam SE, Veronese P, et al. (2011). The COP9 signalosome controls jasmonic acid synthesis and plant responses to herbivory and pathogens. Plant J; 65:480-91.

222. Pelgrom AJE, Meisrimler C-N, Elberse J, et al. (2019). Host targets of effectors of the lettuce downy mildew Bremia lactucae from cDNA-based yeast two-hybrid screening. Cold Spring Harbor Laboratory.

223. Nordgård O, Dahle $\varnothing$, Andersen T $\varnothing$, Gabrielsen OS. (2001). JAB1/CSN5 interacts with the GAL4 DNA binding domain: A note of caution about two-hybrid interactions. 83:969-971.

224. Feiz L, Williams-Carrier R, Belcher S, et al. (2014). A protein with an inactive pterin-4a-carbinolamine dehydratase domain is required for Rubisco biogenesis in plants. The Plant Journal; 80:862-869.

225. Hickman R, Van Verk MC, Van Dijken AJH, et al. (2017). Architecture and Dynamics of the Jasmonic Acid Gene Regulatory Network. The Plant Cell; 29:2086.

226. Neuser J, Metzen CC, Dreyer BH, et al. (2019). HBI1 Mediates the Tradeoff between Growth and Immunity through Its Impact on Apoplastic ROS Homeostasis. Cell Reports; 28:1670-1678.

227. Hill JR, Morris DR. (1993). Cell-specific translational regulation of S-adenosylmethionine decarboxylase mRNA. Dependence on translation and coding capacity of the cis-acting upstream open reading frame. Journal of Biological Chemistry; 268:726-731.

228. Hayden CA, Jorgensen RA. (2007). Identification of novel conserved peptide uORF homology groups in Arabidopsis and rice reveals ancient eukaryotic origin 
of select groups and preferential association with transcription factor-encoding genes. BMC Biology; 5:32.

229. Marco F, Busó E, Carrasco P. (2014). Overexpression of SAMDC1 gene in Arabidopsis thaliana increases expression of defense-related genes as well as resistance to Pseudomonas syringae and Hyaloperonospora arabidopsidis [Original Research]. Frontiers in Plant Science; 5.

230. Gao D, Huibers RP, Loonen AE, et al. (2014). Down-regulation of acetolactate synthase compromises Ol-1- mediated resistance to powdery mildew in tomato. BMC Plant Biology; 14:32.

231. YtterbergAJ, Peltier J-B, van Wijk KJ. (2006). Protein Profiling of Plastoglobules in Chloroplasts and Chromoplasts. A Surprising Site for Differential Accumulation of Metabolic Enzymes. Plant Physiology; 140:984.

232. Hino T, Tanaka Y, Kawamukai M, et al. (2011). Two Sec13p homologs, AtSec13A and AtSec13B, redundantly contribute to the formation of COPII transport vesicles in Arabidopsis thaliana. Bioscience, biotechnology, and biochemistry; 75:1848-1852.

233. Huibers RP, Van den Ackerveken G. (2008). Genetic analysis of susceptibility of Arabidopsis to downy mildew infection Thesis. Utrecht University.

234. Kerk D, Bulgrien J, Smith DW, Gribskov M. (2003). Arabidopsis Proteins Containing Similarity to the Universal Stress Protein Domain of Bacteria. Plant Physiology; 131:1209.

235. Karimi M, Inzé D, Depicker A. (2002). GATEWAY ${ }^{\mathrm{TM}}$ vectors for Agrobacteriummediated plant transformation. Trends in plant science; 7:193-195.

236. Fromont-Racine M, Rain J-C, Legrain P. Building protein-protein networks by two-hybrid mating strategy. Elsevier; 2002. p. 513-524.

237. Kamoun $\mathbf{S}$. The secretome of plant-associated fungi and oomycetes. Plant relationships: Springer; 2009. p. 398.

238. Stassen JH, Van den Ackerveken G. (2011). How do oomycete effectors interfere with plant life? Current opinion in plant biology; 14:407-414.

239. Pecrix Y, Buendia L, Penouilh-Suzette C, et al. (2019). Sunflower resistance to multiple downy mildew pathotypes revealed by recognition of conserved effectors of the oomycete Plasmopara halstedii. Plant J; 97:730-748.

240. Liu Y, Lan X, Song S, et al. (2018). In Planta Functional Analysis and Subcellular Localization of the Oomycete Pathogen Plasmopara viticola Candidate RXLR Effector Repertoire. Frontiers in Plant Science; 9.

241. Xu C, Jiao C, Sun H, et al. (2017). Draft genome of spinach and transcriptome diversity of 120 Spinacia accessions. Nature Communications; 8:15275.

242. van der Schoot C, Paul LK, Paul SB, Rinne PL. (2011). Plant lipid bodies and cell-cell signaling: a new role for an old organelle? Plant signaling \& behavior; 6:1732-1738. 
243. Dagdas YF, Pandey P, Tumtas Y, et al. (2018). Host autophagy machinery is diverted to the pathogen interface to mediate focal defense responses against the Irish potato famine pathogen. Elife; $7: \mathrm{e} 37476$.

244. Jürgens G. (2004). Membrane trafficking in plants. Annu Rev Cell Dev Biol; 20:481-504.

245. Nelson BK, Cai X, Nebenführ A. (2007). A multicolored set of in vivo organelle markers for co-localization studies in Arabidopsis and other plants. The Plant Journal; 51:1126-1136.

246. Saint-Jore-Dupas C, Nebenfuhr A, Boulaflous A, et al. (2006). Plant N-glycan processing enzymes employ different targeting mechanisms for their spatial arrangement along the secretory pathway. Plant Cell; 18:3182-200.

247. Köhler RH, Zipfel WR, Webb WW, Hanson MR. (1997). The green fluorescent protein as a marker to visualize plant mitochondria in vivo. The Plant Journal; 11:613-621.

248. Hooper CM, Castleden IR, Tanz SK, et al. (2016). SUBA4: the interactive data analysis centre for Arabidopsis subcellular protein locations. Nucleic Acids Research; 45:D1064-D1074.

249. Reddy ASN, Day IS, Göhring J, Barta A. (2012). Localization and Dynamics of Nuclear Speckles in Plants. Plant Physiology; 158:67.

250. Üstün S, Hafrén A, Liu Q, et al. (2018). Bacteria ExploitAutophagy for Proteasome Degradation and Enhanced Virulence in Plants. The Plant Cell; 30:668.

251. Merkle T. (2003). Nucleo-cytoplasmic partitioning of proteins in plants: implications for the regulation of environmental and developmental signalling. Current Genetics; 44:231-260.

252. Bohnsack MT, Regener K, Schwappach B, et al. (2002). Exp5 exports eEF1A via tRNA from nuclei and synergizes with other transport pathways to confine translation to the cytoplasm. The EMBO journal; 21:6205-6215.

253. Amaro TMMM, Thilliez GJA, Motion GB, Huitema E. (2017). A Perspective on CRN Proteins in the Genomics Age: Evolution, Classification, Delivery and Function Revisited [Review]. Frontiers in Plant Science; 8:99.

254. Petre B, Lorrain C, Saunders DGO, et al. (2016). Rust fungal effectors mimic host transit peptides to translocate into chloroplasts. Cellular Microbiology; 18:453-465.

255. Wirthmueller L, Roth C, Fabro G, et al. (2015). Probing formation of cargo/ importin $\square$ a transport complexes in plant cells using a pathogen effector. The Plant Journal; 81:40-52.

256. Chen T, Liu R, Dou M, et al. (2020). Insight Into Function and Subcellular Localization of Plasmopara viticola Putative RxLR Effectors. Frontiers in microbiology; 11:692-692.

257. Sperschneider J, Catanzariti A-M, DeBoer K, et al. (2017). LOCALIZER: subcellular localization prediction of both plant and effector proteins in the plant cell. Scientific Reports; 7:44598. 
258. Almagro Armenteros JJ, Salvatore M, Emanuelsson O, et al. (2019). Detecting sequence signals in targeting peptides using deep learning. Life Science Alliance; 2:e201900429.

259. Ciuffetti LM, Manning VA, Pandelova I, et al. (2010). Host $\square$ selective toxins, Ptr ToxA and Ptr ToxB, as necrotrophic effectors in the Pyrenophora tritici $\square$ repentis-wheat interaction. New Phytologist; 187:911-919.

260. Wawra S, Trusch F, Matena A, et al. (2017). The RxLR motif of the host targeting effector AVR3a of Phytophthora infestans is cleaved before secretion. Plant Cell; 29:1184-1195.

261. Singh DK, McNellis TW. (2011). Fibrillin protein function: The tip of the iceberg? Trends in Plant Science; 16:432-441.

262. van Wijk KJ, Kessler F. (2017). Plastoglobuli: Plastid Microcompartments with Integrated Functions in Metabolism, Plastid Developmental Transitions, and Environmental Adaptation. Annual Review of Plant Biology; 68:253-289.

263. Kretschmer M, Damoo D, Djamei A, Kronstad J. (2020). Chloroplasts and Plant Immunity: Where Are the Fungal Effectors? Pathogens; 9:19.

264. Leitner-Dagan Y, Ovadis M, Shklarman E, et al. (2006). Expression and Functional Analyses of the Plastid Lipid-Associated Protein CHRC Suggest Its Role in Chromoplastogenesis and Stress. Plant Physiology; 142:233.

265. Margaria P, Palmano S. (2011). Response of the Vitis vinifera L. cv. 'Nebbiolo' proteome to Flavescence dorée phytoplasma infection. PROTEOMICS; 11:212224.

266. Youssef A, Laizet Yh, Block MA, et al. (2010). Plant lipid-associated fibrillin proteins condition jasmonate production under photosynthetic stress. The Plant Journal; 61:436-445.

267. Koornneef A, Pieterse CMJ. (2008). Cross Talk in Defense Signaling. Plant Physiology; 146:839.

268. Maruyama-Nakashita A, Inoue E, Watanabe-Takahashi A, et al. (2003). Transcriptome profiling of sulfur-responsive genes in Arabidopsis reveals global effects of sulfur nutrition on multiple metabolic pathways. Plant physiology; 132:597-605.

269. Garcia-Molina A, Altmann M, Alkofer A, et al. (2017). LSU network hubs integrate abiotic and biotic stress responses via interaction with the superoxide dismutase FSD2. Journal of Experimental Botany; 68:1185-1197.

270. Spoel SH, Loake GJ. (2011). Redox-based protein modifications: the missing link in plant immune signalling. Current Opinion in Plant Biology; 14:358-364.

271. Olsen AN, Ernst HA, Leggio LL, Skriver K. (2005). NAC transcription factors: structurally distinct, functionally diverse. Trends in plant science; 10:79-87.

272. Yuan X, Wang H, Cai J, et al. (2019). NAC transcription factors in plant immunity. Phytopathology Research; 1:3. 
273. Liang M, Li H, Zhou F, et al. (2015). Subcellular Distribution of NTL Transcription Factors in Arabidopsis thaliana. Traffic; 16:1062-1074.

274. Fan M, Bai M-Y, Kim J-G, et al. (2014). The bHLH transcription factor HBI1 mediates the trade-off between growth and pathogen-associated molecular pattern-triggered immunity in Arabidopsis. The Plant Cell; 26:828-841.

275. Golovkin M, Reddy ASN. (1999). An SC35-like Protein and a Novel Serine/ Arginine-rich Protein Interact with Arabidopsis U1-70K Protein. Journal of Biological Chemistry; 274:36428-36438.

276. Reddy ASN, Shad Ali G. (2011). Plant serine/arginine-rich proteins: roles in precursor messenger RNA splicing, plant development, and stress responses. WIREs RNA; 2:875-889.

277. Aravind L, Anantharaman V, Koonin EV. (2002). Monophyly of class I aminoacyl tRNA synthetase, USPA, ETFP, photolyase, and PP-ATPase nucleotide-binding domains: implications for protein evolution in the RNA world. Proteins; 48:1-14.

278. Nyström T, Neidhardt FC. (1994). Expression and role of the universal stress protein, UspA, of Escherichia coli during growth arrest. Mol Microbiol; 11:537544.

279. Jung YJ, Melencion SMB, Lee ES, et al. (2015). Universal Stress Protein Exhibits a Redox-Dependent Chaperone Function in Arabidopsis and Enhances Plant Tolerance to Heat Shock and Oxidative Stress [Original Research]. Frontiers in Plant Science; 6:1141.

280. Dezfulian MH, Foreman C, Jalili E, et al. (2017). Acetolactate synthase regulatory subunits play divergent and overlapping roles in branched-chain amino acid synthesis and Arabidopsis development. BMC Plant Biology; 17:71.

281. Winter G, Todd CD, Trovato M, et al. (2015). Physiological implications of arginine metabolism in plants [Review]. Frontiers in Plant Science; 6:534.

282. Zeidler D, Zahringer U, Gerber I, et al. (2004). Innate immunity in Arabidopsis thaliana: lipopolysaccharides activate nitric oxide synthase (NOS) and induce defense genes. Proc Natl Acad Sci U S A; 101:15811-6.

283. Umate P. (2012). Comparative Genomics of the Lipid-body-membrane Proteins Oleosin, Caleosin and Steroleosin in Magnoliophyte, Lycophyte and Bryophyte. Genomics, Proteomics \& Bioinformatics; 10:345-353.

284. Collins K, Zhao K, Jiao C, et al. (2019). SpinachBase: a central portal for spinach genomics. Database (Oxford); 2019.

285. Edgar RC. (2004). MUSCLE: multiple sequence alignment with high accuracy and high throughput. Nucleic Acids Res; 32:1792-7.

286. Almagro Armenteros JJ, Sønderby CK, Sønderby SK, et al. (2017). DeepLoc: prediction of protein subcellular localization using deep learning. Bioinformatics; 33:3387-3395.

287. Jones JD, Dangl JL. (2006). The plant immune system. Nature; 444:323-9. 
288. Fawke S, Doumane M, Schornack S. (2015). Oomycete interactions with plants: infection strategies and resistance principles. Microbiol Mol Biol Rev; 79:263-80.

289. Boevink PC, Birch PRJ, Turnbull D, Whisson SC. (2020). Devastating intimacy: the cell biology of plant-Phytophthora interactions. New Phytologist; n/a.

290. Spanu PD. (2012). The genomics of obligate (and nonobligate) biotrophs. Annu Rev Phytopathol; 50:91-109.

291. Judelson HS, Blanco FA. (2005). The spores of Phytophthora: weapons of the plant destroyer. Nat Rev Microbiol; 3:47-58.

292. Jiang RH, Tyler BM. (2012). Mechanisms and evolution of virulence in oomycetes. Annu Rev Phytopathol; 50:295-318.

293. Oliver RP, Solomon PS. (2010). New developments in pathogenicity and virulence of necrotrophs. Current opinion in plant biology; 13:415-419.

294. Pandaranayaka EPJ, Frenkel O, Elad Y, et al. (2019). Network analysis exposes core functions in major lifestyles of fungal and oomycete plant pathogens. BMC Genomics; 20:1020.

295. Duplessis S, Cuomo CA, Lin Y-C, et al. (2011). Obligate biotrophy features unraveled by the genomic analysis of rust fungi. Proceedings of the National Academy of Sciences; 108:9166-9171.

296. Spanu PD, Abbott JC, Amselem J, et al. (2010). Genome expansion and gene loss in powdery mildew fungi reveal tradeoffs in extreme parasitism. Science; 330:1543-6.

297. Casadevall A. (2008). Evolution of intracellular pathogens. Annu Rev Microbiol; 62:19-33.

298. McCutcheon JP, Moran NA. (2012). Extreme genome reduction in symbiotic bacteria. Nat Rev Microbiol; 10:13-26.

299. Parlange F, Oberhaensli S, Breen J, et al. (2011). A major invasion of transposable elements accounts for the large size of the Blumeria graminis f.sp. tritici genome. Functional \& Integrative Genomics; 11:671-677.

300. Croll D, McDonald BA. (2012). The accessory genome as a cradle for adaptive evolution in pathogens. PLOS pathogens; 8.

301. Kämper J, Kahmann R, Bölker M, et al. (2006). Insights from the genome of the biotrophic fungal plant pathogen Ustilago maydis. Nature; 444:97-101.

302. Schirawski J, Mannhaupt G, Münch K, et al. (2010). Pathogenicity determinants in smut fungi revealed by genome comparison. Science; 330:1546-1548.

303. Mooi R. (2009). Evolution, Second Edition. Douglas J. Futuyma. Integrative and Comparative Biology; 49:722-723.

304. Freeman S, Herron JC. Evolutionary analysis. Pearson Prentice Hall Upper Saddle River, NJ; 2007. 
305. Villa NO, Kageyama K, Asano T, Suga H. (2006). Phylogenetic relationships of Pythium and Phytophthora species based on ITS rDNA, cytochrome oxidase II and $\beta$-tubulin gene sequences. Mycologia; 98:410-422.

306. Cooke DE, Drenth A, Duncan JM, et al. (2000). A molecular phylogeny of Phytophthora and related oomycetes. Fungal Genet Biol; 30:17-32.

307. van der Hoorn RA, Kamoun S. (2008). From guard to decoy: a new model for perception of plant pathogen effectors. The Plant Cell; 20:2009-2017.

308. Vleeshouwers VGAA, Oliver RP. (2014). Effectors as tools in disease resistance breeding against biotrophic, hemibiotrophic, and necrotrophic plant pathogens. Mol Plant-Microbe Interact; 27:196-206.

309. Zheng X, McLellan H, Fraiture M, et al. (2014). Functionally redundant RXLR effectors from Phytophthora infestans act at different steps to suppress early flg22-triggered immunity. PLOS pathogens; 10.

310. Vleeshouwers VG, Rietman H, Krenek P, et al. (2008). Effector genomics accelerates discovery and functional profiling of potato disease resistance and phytophthora infestans avirulence genes. PLOS ONE; 3:e2875.

311. Flor HH. Identification of races of flax rust by lines with single rust-conditioning genes. US Dept. of Agriculture; 1954.

312. Laugé R, Joosten MH, Haanstra JP, et al. (1998). Successful search for a resistance gene in tomato targeted against a virulence factor of a fungal pathogen. Proceedings of the National Academy of Sciences; 95:9014-9018.

313. Xing S, Wallmeroth N, Berendzen KW, Grefen C. (2016). Techniques for the analysis of protein-protein interactions in vivo. Plant physiology; 171:727-758.

314. van Schie CC, Takken FL. (2014). Susceptibility genes 101: how to be a good host. Annual review of phytopathology; 52:551-581.

315. Lapin D, Van den Ackerveken G. (2013). Susceptibility to plant disease: more than a failure of host immunity. Trends in Plant Science; 18:546-554.

316. Chen L, Shiotani K, Togashi T, et al. (2010). Analysis of the Rac/Rop Small GTPase Family in Rice: Expression, Subcellular Localization and Role in Disease Resistance. Plant and Cell Physiology; 51:585-595.

317. Poraty-Gavra L, Zimmermann P, Haigis S, et al. (2013). The Arabidopsis Rho of Plants GTPase AtROP6 Functions in Developmental and Pathogen Response Pathways. Plant Physiology; 161:1172.

318. van Damme M, Zeilmaker T, Elberse J, et al. (2009). Downy mildew resistance in Arabidopsis by mutation of HOMOSERINE KINASE. The Plant Cell; 21:21792189.

319. Chen L-Q, Qu X-Q, Hou B-H, et al. (2012). Sucrose Efflux Mediated by SWEET Proteins as a Key Step for Phloem Transport. Science; 335:207.

320. Chen L-Q, Hou B-H, Lalonde S, et al. (2010). Sugar transporters for intercellular exchange and nutrition of pathogens. Nature; 468:527-532.

321. Pel MJ, Wintermans PC, Cabral A, et al. (2014). Functional analysis of 
Hyaloperonospora arabidopsidis RXLR effectors. PLOS ONE; 9.

322. Taylor NJ, Fauquet CM. (2002). Microparticle bombardment as a tool in plant science and agricultural biotechnology. DNA and cell biology; 21:963-977.

323. Ah-Fong AM, Judelson HS. (2011). Vectors for fluorescent protein tagging in Phytophthora: tools for functional genomics and cell biology. Fungal biology; 115:882-890.

324. Huitema E, Smoker M, Kamoun S. A straightforward protocol for electrotransformation of Phytophthora capsici zoospores. Plant Immunity: Springer; 2011. p. 129-135.

325. Ah-Fong AMV, Kagda M, Judelson HS. Illuminating Phytophthora Biology with Fluorescent Protein Tags. In: Ma W, Wolpert T, editors. Plant Pathogenic Fungi and Oomycetes: Methods and Protocols. New York, NY: Springer New York; 2018. p. 119-129. 


\section{Summary}

Peronospora effusa $(P f s)^{*}$ is the most important pathogen of cultivated spinach worldwide. It is an obligate biotrophic oomycete that has a filamentous appearance and grows solely on spinach plants. The visible symptoms include irregular chlorotic spots and gray to brown sporangial masses, primarily on the underside of the leaf. Specialized hyphae form 'haustoria' that penetrate the plant cell walls and form the site of interaction between pathogen and plant.

During infection, oomycete pathogens are known to secrete proteins called effectors. Effectors promote infection by suppressing the plant immune system and manipulation of other plant cell processes. Secreted effectors can either function in the plant tissue (apoplast) or be translocated into plant cells and function there. These host-translocated effectors manipulate processes as broad as immune-and hormone signaling, transcription/translation, production of ROS, and vesicular trafficking (Chapter 1).

To get a better understanding of the molecular interaction between $P f s$ and its spinach host the genome of the first reference strain, Pfs1, was sequenced, assembled and annotated (Chapter 2). The sampling of Pfs1 material from leaves resulted in co-sampling of other foliar microbes and plant DNA. Hence the retrieved sequences are derived from multiple organisms and can be considered a metagenome. We used the CAT tool to filter sequences that were not derived from Pfs from the genome pre-assembly. The CAT tool calls all ORFs on the contigs of the preassembly and assigns taxonomy based on the annotation of the ORFs. The reads assigned to contigs that preliminary had ORFs that were not derived from Pfs were removed from the assembly. The filtered reads were used to create a clean and improved assembly. The CAT tool can thus be successfully used to filter eukaryotic contigs. The obtained Pfs 1 genome assembly was $32.4 \mathrm{Mb}$ consisting of 8,635 scaffolds ( $k$-mer prediction $36.2 \mathrm{Mb}$ ). The BUSCO analysis estimated an $88.9 \%$ genome completeness. Transcript sequencing of a range of infection time points aided the prediction of a total of 13,277 gene models. 
We compared the translated gene models to those of other plantinfecting oomycete species that have different lifestyles. Specifically, we looked into the proteins that are predicted to be secreted (secretome) by the oomycete and may hence be involved in the infection process. We identified common features in the predicted secretomes of different obligate biotrophic oomycetes, regardless of their phylogenetic distance. Their secretomes are generally smaller, compared to hemi-biotrophic and necrotrophic oomycete species. Based on the presence and absence of functional domains, we observe a reduction in proteins involved in cell wall degradation, in Nep1-like proteins (NLPs), proteins with PAN/apple domains, and host-translocated effectors.

Downy mildew species have two known types of effectors that are translocated into the plant cells during infection, the RxLR and CRN effectors. We found that the genome of Pfs 1 encodes 99 candidate RxLR and $14 \mathrm{CRN}$ effector proteins. By comparing them to the proteins of other species we found that 39 candidate effectors are specific to Pfs, 10 to downy mildew species, 23 to both downy mildew and Phytophthora species, and 40 are found in more distantly related species like Pythium and/or Albugo. Generally, effector repertoires of different species are highly variable, possibly as a result of positive selection imposed by the plant immune system. Especially, the C-terminal effector domain, that is thought to be responsible for effector function inside plant cells, is often highly variable. Nevertheless, a small number of RxLR effectors are conserved among phytopathogenic oomycetes, possibly because they contribute significantly to virulence. These effectors may target conserved interacting plant proteins that are involved in the infection process and contribute to plant disease susceptibility. The identification of Arabidopsis proteins that interact with Pfs effectors supports the idea of conserved plant targets across species (Chapter $\mathbf{3}$ ). 
One of the more conserved Pfs RxLR proteins showed similarity to the Hyaloperonospora arabidopsidis (Hpa) HaRxL44 effector. HaRxL44 is known to interact and degrade Mediator Subunit 19a, thereby enhancing ethylene and jasmonic acid signaling and ultimately suppressing salicylic acid-triggered immunity. Also, we found that seven of the CRN proteins group into one family that has expanded within the Pfs genome. These proteins are phylogenetically related to a group of Hpa CRN effectors that are remarkable because they have a RxLRLFLAK motif, that shows characteristics of both the RxLR and LFLAK putative translocation domains.

A total of 33 candidate RxLR and CRN effectors of Pfs 1 were cloned and used as baits in a yeast two-hybrid screen to identify interacting plant proteins from an Arabidopsis oligo-dT primed cDNA library. In these assays the first 46 plant proteins that interact with RxLR and CRN effectors of Pfs were identified. A protein interaction network was created with the identified proteins and supplemented with reported protein-protein interactions from the plant-pathogen literature. We found that nine interactors of $P f s$ effectors were targeted by effectors from other bacterial, fungal and/or oomycete pathogens. These highly connected plant proteins like CSN5A, TCP14, and FBN1A likely play important regulatory roles in the plant cell during infection. Other Pfs effectors interact with less connected proteins. Some of these, like HBI1, two NAC transcription factors and two prenylated Rab acceptor (PRA1) proteins, have been linked to disease susceptibility in other plant species before but for most of them their biological function is unknown.

Eight candidate effectors and their fourteen interacting plant proteins were selected to further study their interaction in planta (Chapter 4). The spinach orthologs of the Arabidopsis proteins were identified and the subcellular localization of the candidate effectors, interacting proteins and spinach orthologs was assessed using confocal microscopy. The effector proteins were found to localize to the nucleus, cytoplasm, ER and punctate structures. All but two of the 14 tested Arabidopsis interactors and their spinach orthologs localized with the candidate effector in the same subcellular compartment. The subcellular localization of six plant 
interactors and two effector proteins was altered upon co-expression of the effector and interactor proteins. Furthermore, seven plant proteins showed a more stable signal when co-expressed with the effector. Further studies are needed to confirm the interaction between the proteins and assess the role of the plant proteins in disease susceptibility or resistance.

The general discussion is focused on the genetic differences between saprotrophic and plant pathogenic species and explores the dynamics behind the remarkable small genomes of downy mildew species (including Pfs) (Chapter 5). Also, the unresolved taxonomic issues in the large and diverse taxa of Pythiaceae and Peronosporales are discussed. Finally, we supply the interspecies effector conservation data (Chapter 3) with data of the variation of effectors between races of Pfs to identify interesting targets for further research and breeding. Taken together, the study of conserved effectors and their interacting plant proteins provides a great opportunity to understand the molecular interaction between plants and pathogens and to translate that knowledge to resistance breeding.

${ }^{*}$ Pfs was previously known as Peronospora farinosa forma specialis spinaciae 


\section{Samenvatting}

Peronospora effusa $(P f s)^{*}$, of valse meeldauw, is wereldwijd de meest voorkomende ziekte van het spinazie. Pfs is een oomyceet met een schimmelachtig voorkomen die uitsluitend groeit op levende planten. De zichtbare symptomen van de infectie zijn onregelmatige lichte vlekken op de bladeren en een bruine tot grijze 'vacht' van sporendragers en sporen dat voornamelijk zichtbaar is aan de onderkant van de bladeren.

De hyfen groeien in het plantenweefsel tussen de cellen, waar gespecialiseerde structuren door de celwanden van de planten heen breken en haustoria vormen. Haustoria doen dienst als platform waar uitwisseling van moleculaire componenten tussen de oomyceet en plant plaatsvindt. Oomyceten scheiden tijdens de infectie eiwitten uit die effectoren genoemd worden. Effectoren kunnen actief zijn in het plantenweefsel tussen de cellen of over de celmembraan van de plantencel worden getransporteerd. Effectoren dragen bij aan de infectie door het immuunsysteem van de plant te onderdrukken en door diverse celprocessen te beïnvloeden. Voorbeelden hiervan zijn beïnvloeding van transcriptie/translatie-gerelateerde processen, het verstoren van de hormoonbalans, de productie van reactieve zuurstof die een rol spelen bij de afweerreactie en het transport van eiwitten (Hoofdstuk 1).

Om een beter beeld te krijgen van de moleculaire interactie tussen $P f s$ en spinazie hebben we het genoom van het referentieisolaat Pfs1 in kaart gebracht (Hoofdstuk 2). Het verkrijgen van DNA van Pfs werd bemoeilijkt doordat Pfs uitsluitend op levende planten kan groeien. Het verzamelde materiaal bevatte daarom naast DNA van Pfs ook DNA van andere micro-organismen die op het blad voorkomen. $\mathrm{Er}$ werd een filteringstap toegepast om de verkregen 'DNA reads' en daaruit samengestelde 'DNA contigs' te selecteren op basis van hun herkomst. De filtering werd gedaan met behulp van de CAT tool. Deze methode identificeert de open leesramen (ORFs), of genen, op elk contig en bepaald de taxonomische herkomst hiervan. De contigs met overwegend ORFs die niet afkomstig zijn van Pfs werden geïdentificeerd en uit de DNA set gefilterd. Deze studie is de eerste die laat zien dat de CAT tool 
succesvol eukaryote genomen kan filteren. Het verkregen Pfs1 genoom had een grootte van $32.4 \mathrm{Mb}$ en bestond uit 8,635 scaffolds ( $k$-mer voorspelling $36.2 \mathrm{Mb}$ ). Naar schatting is het genoom voor $88.9 \%$ compleet (BUSCO). Met behulp van RNA, geïsoleerd van samples verzameld op verschillende infectietijdspunten, werden 13,277 genmodellen voorspeld op het genoom.

De eiwitten die gecodeerd worden door bovengenoemde genen werden vergeleken met de eiwitten van andere plant-infecterende oomyceten met uiteenlopende levensstijlen. Hierbij lag de nadruk op de eiwitten die mogelijk door de oomyceten worden uitgescheiden, het secretoom, omdat deze mogelijk een rol spelen in het infectieproces. We ontdekten dat de secretomen van soorten die uitsluitend op levende planten kunnen groeien opmerkelijke overeenkomsten hadden, onafhankelijk van hun fylogenetische afstand tot elkaar. De secretomen van obligaat-biotrofe soorten waren over het algemeen kleiner dan die van hemi-biotrofe of necrotrofe soorten. Gebaseerd op de aanwezigheid van bekende functionele eiwitdomeinen vonden we een lagere abundantie van gesecreteerde eiwitten die betrokken zijn bij celwandafbraak, van Nep1-like proteins (NLPs) en van eiwitten met PAN/apple domeinen alsmede van effectoren die functioneren in plantencellen.

Valse meeldauw soorten hebben twee typen effectoren die in de plantencel terechtkomen tijdens de infectie. Deze RxLR en CRN effectoren interacteren met planteneiwitten om celprocessen te beïnvloeden. In het genoom van Pfs1 hebben we 99 RxLR en 14 CRN kandidaat effectoren gevonden. In een vergelijking met andere soorten zagen we dat 39 effectoren specifiek zijn voor $P f s$. Voor de andere effectoren zijn verwante eiwitten gevonden in andere soorten. Voor 10 effectoren zijn er alleen verwante eiwitten in andere valse meeldauwsoorten, voor 23 zijn er verwanten in zowel andere valse meeldauwsoorten als in Phytophthora soorten en de laatste 40 effectoren zijn daarnaast ook geconserveerd in de minder verwante Pythium en/of Albugo soorten (Hoofdstuk 3).

In de regel zijn er weinig overeenkomsten tussen de effectorrepertoires van verschillende soorten, mogelijk doordat de effectoren onder evolutionaire druk staan om herkenning van het 
plantenimmuunsysteem te voorkomen. Met name het effector domein dat verantwoordelijk is voor de functie van effectoreiwitten is vaak sterk variabel. Ondanks deze trend zijn er een aantal RxLR effectoren bekend die voorkomen in meerdere soorten en sterke overeenkomsten vertonen. Deze effectoren zijn mogelijk behouden gebleven doordat ze een belangrijke rol hebben in het infectieproces. Mogelijk interacteren deze effectoren met geconserveerde planteneiwitten die een rol spelen in de vatbaarheid van de plant voor de ziekte.

Zo hebben we in $P f s$ een effector gevonden die vergelijkbaar is met Hyaloperonospora arabidopsidis effector HaRxL44. HaRxL44 induceert de afbraak van Mediator Subunit 19a. De afbraak leidt tot een toename in ethyleen en jasmonzuur en daarmee indirect tot een onderdrukking van de immune reactie die gereguleerd wordt door salicylzuur. Daarnaast liet het vergelijkingsonderzoek zien dat zeven van de CRN effectoren van $P f s$ aan elkaar verwant zijn en zijn ontstaan binnen Pfs. Fylogenetisch zijn ze onder anderen verwant aan een opmerkelijke groep CRN effectoren van Hpa die zowel een mogelijk RxLR als een LFLAK (CRN) motief hebben. Deze motieven zijn karakteristiek voor de RxLR en CRN effectoren en spelen mogelijk een rol in het transport van de eiwitten over het membraan van de plantencel.

Drieëndertig kandidaat Pfs effectoren werden gekloneerd en gebruikt als 'bait' in een 'yeast two-hybrid' studie. De effectoren werden gebruikt om interacterende eiwitten uit een verzameling van Arabidopsis eiwitten te selecteren (vanuit een "oligo-dT primed yeast two-hybrid cDNA library"). In totaal werden er 46 interacties gevonden tussen planteneiwitten en 18 effectoren. De gevonden planteneiwitten gelden als de eerste $P f s$-interacterende planteneiwitten ooit geïdentificeerd. De interacties werden weergegeven in een interactienetwerk met daarin ook interacterende planteneiwitten geïdentificeerd met effectoren van andere soorten. Het netwerk liet zien dat negen van de eiwitten die interacteerde met $P f s$ effectoren ook interacties aangingen met effectoren van andere (bacteriële, schimmel, of oomyceet) ziekteverwekkers. Deze sterk verbonden eiwitten, zoals CSN5a, TFP14 en FBN1A, spelen mogelijk een belangrijke regulerende rol in de plantencel tijdens de infectie. Een aantal 238 van de gevonden eiwitten zoals HBI1 en twee NAC transcriptiefactoren, 
werden al eerder in verband gebracht met ziektegevoeligheid in andere soorten maar voor de meeste van de 46 eiwitten is de biologische functie nog niet bekend.

Acht effectoren en veertien interacterende Arabidopsis eiwitten werden uitgekozen voor verder onderzoek (Hoofdstuk 4). Voor deze Arabidopsis eiwitten werden de spinazie orthologen geïdentificeerd en meegenomen in het onderzoek. De effectoren en planteneiwitten werd gefuseerd met fluorescente eiwitten en de locatie in de plantencel werd in kaart gebracht met behulp van confocale microscopie. De effectoreiwitten waren zichtbaar in verschillende cellulaire locaties zoals de celkern, het cytoplasma, het ER en als kleine spikkels verspreid door de cel. Twaalf van de veertien Arabidopsis eiwitten en hun spinazie orthologen bevonden zich in dezelfde cellulaire compartimenten als de corresponderende effectoren. De locatie van zes planteneiwitten en twee effectoren veranderde wanneer ze tegelijk tot expressie werden gebracht. Daarnaast waren er zeven planteneiwitten waarvan het signaal beter zichtbaar werd in aanwezigheid van de effector. De interactie tussen de eiwitten en de rol van de interacties tijdens de infectie zullen worden bevestigd in toekomstige experimenten.

In de algemene discussie ligt de nadruk op de genetische verschillen tussen saprotrofe en pathogene oomyceten en ga ik verder in op de opmerkelijk kleine genomen van de valse meeldauwsoorten (waaronder Pfs) (Hoofdstuk 5). Daarnaast ga ik in op de taxonomie van de plant infecterende oomyceten en de problemen in de fylogenetische indeling van met name de soorten in de Pythiaceae en Peronosporales. Daarnaast integreer ik de gegevens over de conservering van effectoren tussen soorten (Hoofdstuk 3) met informatie over conservering van effectoren binnen Pfs isolaten. De gecombineerde data leveren een lijst op van interessante kandidaten voor verder onderzoek en voor toepassing bij het veredelen van resistente gewassen.

Het bestuderen van geconserveerde effectoren en de eiwitten waarmee ze interacteren is een unieke kans om inzicht te krijgen in de moleculaire interactie tussen planten en ziekteverwekkers en levert waardevolle informatie op voor de resistentieveredeling. ${ }^{*} P f s$ stond oorspronkelijk bekend als $P$. farinosa forma specialis spinaciae 


\section{Acknowledgement/Dankwoord}

Alle clichés over promoveren zijn waar. Het is leuk om je eigen project te hebben. Het vraagt veel van je. Je leert veel, ook over jezelf. Het is niet altijd makkelijk. Aan het einde zijn er labprotocollen die je midden in de nacht met je ogen dicht en je handen achter je rug kunt uitvoeren. En: je kunt het niet alleen. Met dit dankwoord wil ik graag iedereen bedanken die op wat voor manier dan ook heeft bijgedragen aan mijn ontwikkeling als wetenschapper en als persoon.

Als allereerste wil ik mijn promotor Guido bedanken. Dankzij jou kreeg ik de kans om dit avontuur aan te gaan. Ik zeg wel eens voor de grap dat ik de eerste jaren van mijn project geen idee had wat ik van je kon verwachten. Kwam ik met slecht nieuws dan bleef je altijd positief. Had ik mooie resultaten dan kreeg ik in plaats van complimenten ineens een hele batterij aan kritische vragen! Het was in ieder geval nooit saai. Gelukkig hebben we elkaar beter leren kennen en hebben we nu een prettige samenwerking. Bedankt voor alles dat je hebt gedaan om dit werk mogelijk te maken, ik heb veel van je geleerd. Ik kijk er naar uit om te zien wat voor mooie ontdekkingen je de komende jaren met je vakgroep gaat doen.

Daarnaast wil ik ook de andere Pl's bedanken. Corné, ik heb bewondering voor de manier waarop jij de vakgroep leidt. Je zorgt voor een fijne sfeer en bent altijd constructief. Ik hoop dat je nog eens denkt aan Eline en mij als er iemand met een stapel post-its binnenkomt. Saskia, Peter, Roeland en Ronnie, jullie vormen het fundament van de groep, bedankt voor alle input en steun de afgelopen jaren. Ronnie, bedankt voor al je geduld met mijn eindeloze OrthoMCL en Orthofinder vragen.

Joël, mijn partner in crime. Er waren, zoals je al aankondigde bij mijn sollicitatie, perioden dat ik je meer zag dan mijn gezin. Gelukkig ontdekten we al snel dat bij echt grote stress een grote zak M\&Ms altijd een oplossing is. Ik ben blij met onze samenwerking en het resultaat er van. Bedankt voor al je steun en gezelligheid! Alexandra, bedankt voor je geduld met mij en mijn yeast two hybrid library. Jou kennis en kunde waren altijd een inspiratie voor mij. Claudia, thank you for sharing your 
expertise on proteins and imaging with me and the lab. I think there are only few people that can match your skills and drive. I really enjoyed working with you and look forward to meeting you again!

Over the course of my project I had pleasure to work with some nice and bright master students. Shan, you were the first and did a great job. You are skilled, eager to learn and I enjoyed our conversations. Martijn, ik stond te kijken van jou tempo en efficiëntie. Jou kijk op de dingen en droge humor maakten de dagen vaak een stuk leuker. Kim, je bent een harde werker en erg streng voor je zelf. Jou vrolijkheid en gezelligheid zijn aanstekelijk, je was een aanwinst voor het lab! Zuzanna, you are bright, skilled and driven. Your understanding of the molecular work is a great asset and in enjoyed working with you.

Een speciaal woord van dank voor de technicians van PMI, zonder jullie zou het onderzoek niet mogelijk zijn! Anja, bedankt dat je altijd voor iedereen klaar staat en interesse hebt in de mensen om je heen. Hans, bedankt voor je eindeloze kennis en gezelligheid! Miek, ik vond het altijd fijn als je er was. Een luisterend oor en een hart van goud. Joyce, ik bewonder jou precisie en volhardendheid, ik zou willen dat ik je veel vaker om hulp zou hebben gevraagd! Kim, je was een fijne collega en een harde werker, bedankt voor alle tijd die we samen doorbrachten! Marrit, ik heb je leren kennen als een leuke collega en doortastende technician, je bent een aanwinst voor het team. Frederike, bedankt voor het overnemen van mijn project tijdens mijn verlof en het huiselijk maken van het kantoor.

Tijmen, jou humor, betrokkenheid en enthousiasme zijn geweldig. Je bent een fijne collega en nog een betere vriend! Eline, het begin van onze vriendschap vergeet ik nooit meer; 'Hey Manon, ik heb gezegd dat wij samen het kerstdiner organiseren'. Het was fijn om een onofficiële buddy te hebben. Bedankt voor de fijne tijd! Pim, jou bevlogenheid werkt aanstekelijk en je humor en party-mood zijn fantastisch! Sebastian and Marjolein, thank you for being great office mates! 
Merel, met jou gezelligheid en betrokkenheid zorgde je altijd voor een groepsgevoel. Door jou enthousiasme deed ik mee aan de organisatie van de European Plant Science Retreat. Het bleek één van de leukste activiteiten van mijn PhD. Daardoor leerde ik ook Sjors en Jesse beter kennen. Het was super leuk om een team met jullie en de anderen te vormen en samen een mooi evenement neer te zetten. Gilles, bedankt voor de leuke tijd bij PMI. Hao, it was a pleasure working with you. I admire your kindness, curiosity and willingness to always give us your best! Sarah, I never knew that a possible side effect of becoming a $\mathrm{PhD}$ is to gain an evil twin sister. Thank you for all the great times, you are the best!

If I were to list all current and previous members of PMI that helped, supported and inspired me during my $\mathrm{PhD}$, this acknowledgement section would never end. Hence, as a thank you gesture, I will simply list you all: Niels, Sietske, Shu-hua, Dharani, Xingtong, Sanne, Dario, Juan, Gijs, Yang, Giannis, Aster, Changfeng, Jiayu, Marciel, Richard, Erqin, Ke, Pauline, Tom, Alberto, Colette, Marco, Iván, Marcel, Nora, Lotte, Silvia P., Irene, Yeling, Nicole, Silvia C., Paul and Dongping, your help and company were greatly appreciated! The same goes for Sara Buti, Basten Snoek, Berend Snel and Michael Seidl, colleagues from other groups at UU, thank you! Mijn dank gaat ook uit naar alle mensen die namens de bedrijven betrokken waren bij dit project. Bedankt voor jullie kennis en de waardevolle discussies. Dorine en Lenneke van Maliebaan 45 bedankt voor de fijne werkplek tijdens de Corona-lockdown, het heeft enorm geholpen! Rick, bedankt voor het bijspringen toen de deadline stress het hoogst was!

Ook buiten PMI zijn er veel mensen die een enorme steun zijn geweest in de afgelopen jaren. Ik prijs mezelf gelukkig met grote groep vrienden waarop ik altijd terug kan vallen. Jullie maken het leven leuk. Ik ga me niet wagen aan een lijstje, jullie weten wie jullie zijn! In het bijzonder; Rascha, het is fijn om een vriendin te hebben zoals jij. Bedankt voor je steun en vriendschap! Colene en Meike, bedankt dat jullie er altijd voor me zijn, ik kan me geen betere vriendinnen wensen.

Mijn schoonfamilie en familie zijn ook een grote steun geweest. 
Wilma, Theo, Miranda, Patrick, Ties, Mads en Nora, jullie zijn mijn tweede thuis. Bedankt voor het meeleven en de onvoorwaardelijke steun!

Oma Elly, het was heerlijk om elke dinsdag avond bij $u$ te gaan eten. Bedankt dat $u$ altijd zo meeleefde, het is fijn om $u$ zo dichtbij te hebben. Helaas heeft opa dit niet meer mee kunnen maken maar ik weet zeker dat hij trots op ons zou zijn.

Frank, Alice en Robin, bedankt dat jullie mij als bonus familie hebben opgenomen! Ronny, bedankt voor je hulp en enthousiasme, ik vind het altijd gezellig om bij jullie langs te komen. Boyd, bedankt voor alle gezeligheid!

Mam bedankt dat je er altijd voor me bent. Ik vind het super leuk dat je je best doet om te begrijpen waarmee ik bezig ben. Pap, bedankt voor je betrokkenheid en vermogen om de dingen te relativeren. Suzanne, ik ben zo blij met onze band en vriendschap, jij bent het liefste zusje dat er is. Al laat ik het misschien niet altijd zien, jullie liefde en betrokkenheid betekenen veel voor me!

Lieve Tim, wat er ook speelt op het werk, thuiskomen bij jou maakt het allemaal onbelangrijk. Bedankt voor je eindeloze geduld en begrip. Ik ben zo gelukkig met jou en Anne. Ik hou van jullie! 


\section{About the author}



Manon Neilen was born on 14 March 1990 in Utrecht, the Netherlands. She completed her secondary education, VWO, at Openbare Regionale Scholengemeenschap (ORS) Lek en Linge in Culemborg in 2008. She started her Biology Bachelor at Utrecht University during the same year and obtained her Bachelor of Science degree in 2011. She continued her studies by enrolling in the Environmental Biology Master

at the same University. During her Master studies, Manon carried out two research projects. The first one was at Naturalis Biodiversity Centre within the Fungal Biology group under supervision of Dr. József Geml. In her project she worked on the environmental sequencing of the Salixassociated ectomycorrhizal fungal community in the arctic tundra and Dutch sand dune ecosystems. In her second project she studied the diversity of bacterial and fungal decomposers in agricultural soils in a collaborative project between the institute of risk assessment (IRAS, UU, Dr. Heike Schmitt) and Netherlands institute of ecology (NIOO, Prof. dr. Eiko Kuramae). After her graduation in 2014, Manon worked as an assistant researcher Phytopathology at Enza Zaden in Enkhuizen. In February 2015, she started as a PhD candidate at the Plant-Microbe Interactions group at Utrecht University under the supervision of Prof. dr. Guido van den Ackerveken. During her PhD Manon worked on comparative genomics and host-translocated effectors of the spinach downy mildew, Peronospora effusa. 


\section{List of publications}

Neilen, M., Klein J., van Verk, M., Dutilh, B. E.,\& Van den Ackerveken, G. (2020).

Genome reconstruction of the non-culturable spinach downy mildew Peronospora effusa by metagenome filtering. PLOS ONE; 15.

Thines M., Sharma R., Rodenburg S.Y.A., Gogleva A., Judelson H.S., Xia X., van den Hoogen J., Kitner M., Klein J., Neilen M., de Ridder D., Seidl M.F., van den Ackerveken G., Govers F., Schornack S., and Studholme D.J. (2020). The Genome of Peronospora belbahrii reveals high heterozygosity, a low number of canonical effectors, and TC-rich promoters. Molecular Plant-Microbe Interactions; 33:742-753.

van der Horst S., van Butselaar T., Zhang H., Vismans G., Steenbergen M., Courbier S., Neilen M., Küpers J.J. (2019). Bringing together Europe's young plant scientists. New Phytologist; 222; 29-31.

Geml J., Gravendeel B., van der Gaag K.J., Neilen, M., Lammers Y., et al. (2014). The Contribution of DNA Metabarcoding to Fungal Conservation: Diversity Assessment, Habitat Partitioning and Mapping Red-listed Fungi in Protected Coastal Salix repens Communities in the Netherlands. PLOS ONE; 9. 
